\title{
Enhancing the Thermoelectric Performance of Calcium Cobaltite through Cation Substitution and Non-Stoichiometric Addition
}

Cullen Boyle

Follow this and additional works at: https://researchrepository.wvu.edu/etd

\section{Recommended Citation}

Boyle, Cullen, "Enhancing the Thermoelectric Performance of Calcium Cobaltite through Cation Substitution and Non-Stoichiometric Addition" (2017). Graduate Theses, Dissertations, and Problem Reports. 5247.

https://researchrepository.wvu.edu/etd/5247

This Dissertation is protected by copyright and/or related rights. It has been brought to you by the The Research Repository @ WVU with permission from the rights-holder(s). You are free to use this Dissertation in any way that is permitted by the copyright and related rights legislation that applies to your use. For other uses you must obtain permission from the rights-holder(s) directly, unless additional rights are indicated by a Creative Commons license in the record and/ or on the work itself. This Dissertation has been accepted for inclusion in WVU Graduate Theses, Dissertations, and Problem Reports collection by an authorized administrator of The Research Repository @ WVU.

For more information, please contact researchrepository@mail.wvu.edu. 


\title{
Enhancing the Thermoelectric Performance of Calcium Cobaltite through Cation Substitution and Non- Stoichiometric Addition
}

\section{Cullen Boyle}

Dissertation submitted

to the Benjamin Statler College of Engineering and Mineral Resources at West Virginia University

in partial fulfillment of the requirements for the degree of

Doctor of Philosophy in Mechanical and Aerospace Engineering

Committee members:

Xueyan Song, Ph.D., Chair

Yun Chen, Ph.D. Jacky Prucz Ph.D.

Dustin McIntyre Ph.D.

Hailin Li Ph.D.

Bruce Kang Ph.D.

Department of Mechanical and Aerospace Engineering

\author{
Morgantown, West Virginia
}

2017

Keywords: Thermoelectric, Oxide, Grain Boundary, Segregation

Copyright 2017 Cullen Boyle 


\section{Abstract \\ Enhancing the Thermoelectric Performance of Calcium Cobaltite through Cation Substitution and Non-Stoichiometric Addition \\ Cullen Boyle}

Thermoelectric (TE) materials have the unique ability to convert temperature differences directly into electricity due to the Seebeck effect. Thermoelectric generators have a variety of applications including waste heat recovery for power plants and automobiles. For high-temperature waste-heat recovery, misfit layered calcium cobaltite, $\mathrm{Ca}_{3} \mathrm{C}_{4} \mathrm{O}_{9}$, is one of the most promising $p$-type TE oxides offering good durability in air with low cost and minimized environmental impact. A challenge for developing polycrystalline calcium cobaltite TE materials is to improve its energy conversion efficiency for large scale applications. The energy conversion efficiency of TE materials is characterized using the figure-of-merit $Z T$, which is defined as $Z T=S^{2} \rho^{-1} K^{-1} T$, where $S, \rho, S^{2} \rho^{-1}, K^{-1}$, and T are Seebeck coefficient, electrical resistivity, power factor, thermal conductivity, and temperature respectively. State of the art commercial conventional TE materials, such as bismuth telluride $\left(\mathrm{Bi}_{2} \mathrm{Te}_{3}\right)$ and lead telluride $(\mathrm{PbTe})$, possess values of $Z T \approx 1$, which corresponds to an energy conversion efficiency of $10 \%$. Calcium cobaltite single crystals show extraordinary TE performance with an extrapolated $Z T$ value of 0.87 at $973 \mathrm{~K} .{ }^{4}$ By contrast, the TE performance of polycrystals is reported to be only $\sim 20 \%$ of that from the single crystal and with the commonly measured $Z T$ of $\sim 0.2$ at $\sim 900 \mathrm{~K}$. This dissertation focuses on improving the performance of polycrystalline $\mathrm{Ca}_{3} \mathrm{C}_{4} \mathrm{O}_{9}$ ceramics for large scale applications. The present work demonstrates the feasibility of increasing the values of Seebeck coefficient, $S$ and power factor of calcium cobaltite $\mathrm{Ca}_{3} \mathrm{Co}_{4} \mathrm{O}_{9}$ ceramics through dopant grain boundary (GB) segregation. For the first time in the field of thermoelectrics, various dopants which include $\mathrm{Bi}, \mathrm{Ba}$, and co-dopants $\mathrm{Bi}-\mathrm{Ba}$ were proved to segregate to the GBs and dramatically increased the $\mathrm{ZT}$ of $\mathrm{Ca}_{3} \mathrm{CO}_{4} \mathrm{O}_{9}$, and a high $\mathrm{ZT}$ of 0.52 was achieved for $\mathrm{Ca}_{3} \mathrm{C}_{4} \mathrm{O}_{9}$ ceramics through dopants grain boundary segregation. The method of introducing the dopants to the grain boundaries, and their origin of the performance enhancement of thermoelectric oxide ceramics were thoroughly investigated and presented in this dissertation. 


\section{Acknowledgments}

I would like to extend my greatest thanks to my advisor Dr. Xueyan Song for all of her help throughout my research experience. She believed in me from the day she first approached me to be a part of her research group. She was always interested in my input and took my ideas and concerns seriously which allowed me to grow as a student, researcher, and a person. She taught me how to approach and analyze a situation from the best perspective and to never give up. She was always encouraging and pushing me to reach my potential. She demonstrated and conducted herself in a professional fashion and set an example for me to look up to and something to strive for.

I would like to thank to Dr. Chen for his patience and the knowledge he has bestowed upon me. Thank you for everything you have taught me these past years in the lab including the constant refreshers and reminders necessary for me to fully understand and grasp a concept. Thank you for teaching me patience, dedication, and attention to the finer details.

Thank you to Dr. Victor Mucino for guiding me during the times I did not have an advisor and continuing to help me through this process. You pushed me and gave me someone to bounce ideas off of when I had no one else to consult. When I was new to the area and had no idea how to proceed in the program you gave me direction and helped me to choose my coursework, find my interests, and obtain funding. Thank you for your kind words of wisdom.

I would like to thank my committee members for their valuable time and effort to help guide and advise me through this endeavor.

A very special thank you to my family. I would not be where I am today without your constant encouragement and belief in me. Thank you to my mother and father for the encouragement to go back to school and further my degree, even if it meant going months at a time not seeing you. You were always there to help me see things optimistically when things looked bleak. You gave me someone to talk to when I was feeling frustrated and were always there to reassure me during moments of uncertainty. Your patience and unconditional support throughout my many years of schooling has allowed me to achieve something great that I otherwise would have never thought possible. You taught me the value of hard work and how to be the best version of myself even when the task felt impossible. The morals and beliefs you have instilled in me gave me the fortitude and confidence to follow my interests and dreams and to set goals no matter how difficult the path may seem.

Lastly, I would like to thank God for giving me the capacity to constantly push and better myself no matter the task. For creating a world full of wonder and awe which allows scientist to ask the question 'Why?' and, if driven enough, the ability to find the answers. Without the constant mysteries and perpetual questions of the world we would not have a reason to search for answers and push boundaries in the first place. 


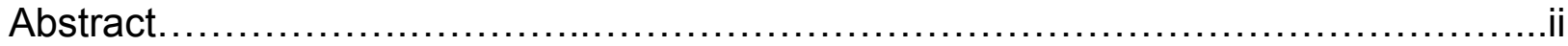

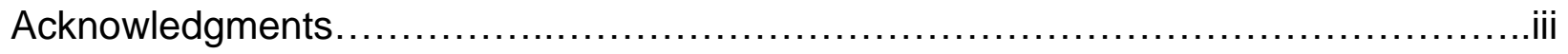

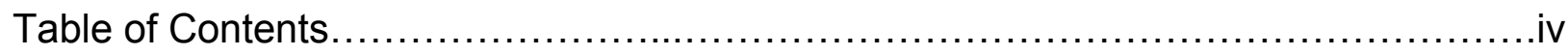

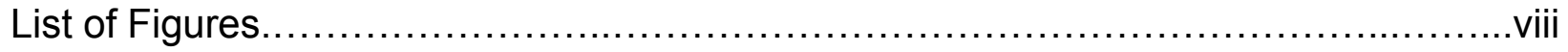

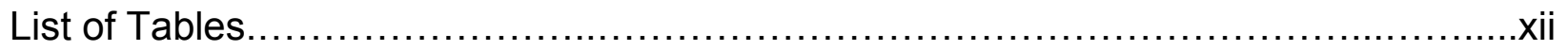

Chapter 1: Background Review of Thermoelectric Generators and Materials ...... 13

1.1 Brief introduction of thermoelectric generators ....................................... 13

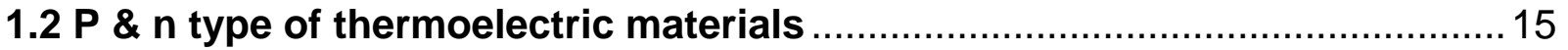

1.3 Figure of Merit ZT and specific ZTs of various materials ............................20

1.4 Physical characteristics of thermoelectric materials …...............................22

1.4.1 Electrical Properties of $\mathrm{p}-\mathrm{\&} \mathrm{n}$ - type materials ............................................ 22

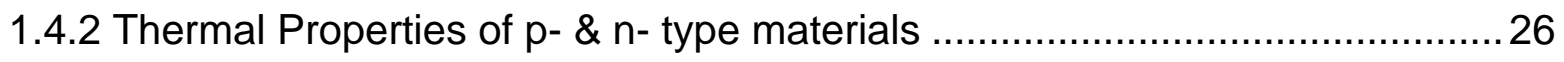

1.5 Oxides and Calcium Cobaltite as Thermoelectric materials .........................29

1.5.1 Use of oxides as Thermoelectric Materials ...............................................29

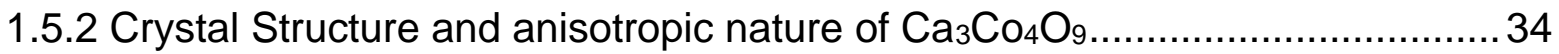

1.5.3 Current Status of $\mathrm{Ca}_{3} \mathrm{Co}_{4} \mathrm{O}_{9}$ Thermoelectric Performance Reported in

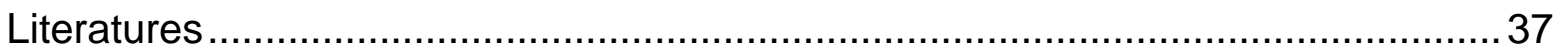

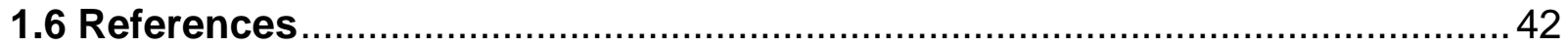

Chapter 2: Research Objectives and Methodology................................................ 47

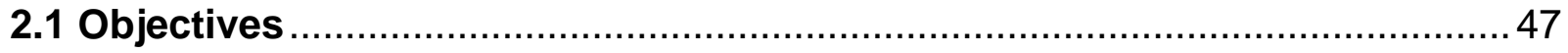

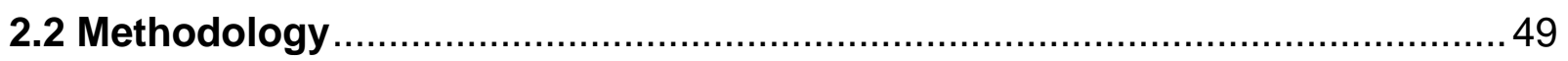

Chapter 3: Effect of Bismuth Telluride Addition on the Electrical Performance of

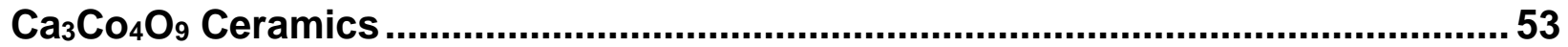

3.1 Background \& motivation of using Bismuth Telluride as a dopant in

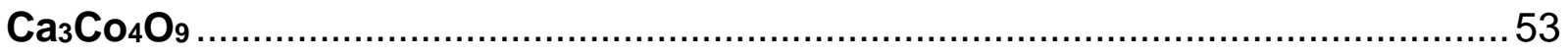

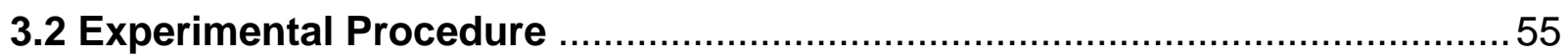

3.3 Electrical Performance of $\mathrm{Ca}_{3} \mathrm{Co}_{4} \mathrm{O}_{9}$ doped with $\mathrm{Bi}_{2} \mathrm{Te}_{3} \ldots \ldots \ldots \ldots \ldots \ldots \ldots \ldots \ldots . . . . . \ldots \ldots$

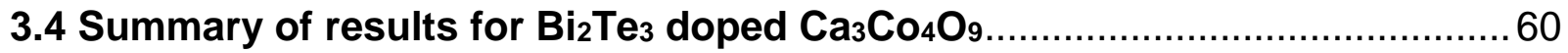

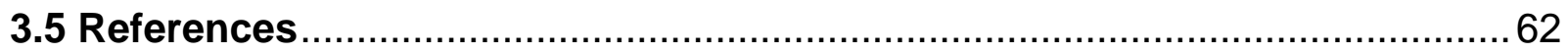

Chapter 4: Improving the TE performance and crystal texture of $\mathrm{Ca}_{3} \mathrm{Co}_{4} \mathrm{O}_{9}$

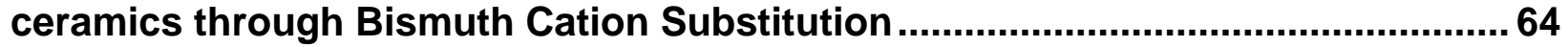

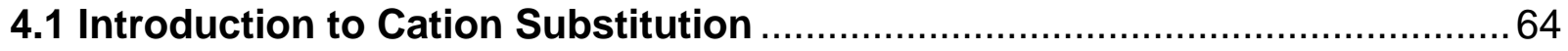


4.2 Background and motivation for Bismuth as a dopant in $\mathrm{Ca}_{3} \mathrm{Co}_{4} \mathrm{O}_{9}$ .64

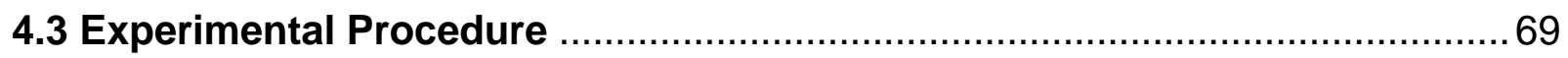

4.4 Experimental Results of Bismuth doped $\mathrm{Ca}_{3} \mathrm{C}_{4} \mathrm{O}_{9}$ using cation substitution

4.4.1 Presence of bismuth in the lattice \& at the grain boundary of $\mathrm{Ca}_{3} \mathrm{Co}_{4} \mathrm{O}_{9}$ ceramics 71

4.4.2 Power factor enhancement of $\mathrm{Ca}_{3} \mathrm{C}_{4} \mathrm{O}_{9}$ through Bismuth Substitution of $\mathrm{Ca} 74$

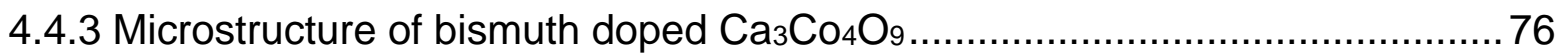

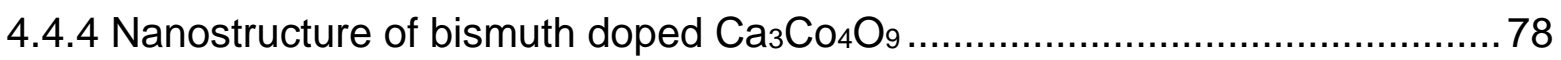

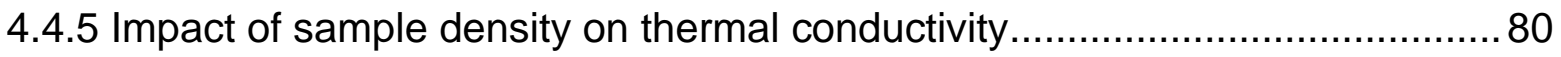
4.4.6 Significance of low thermal conductivity for achieving the high Figure of Merit 82

4.5 Summary of results for Bismuth cation substitution in $\mathrm{Ca}_{3} \mathrm{Co}_{4} \mathrm{O}_{9} \ldots \ldots \ldots \ldots . . . . .84$

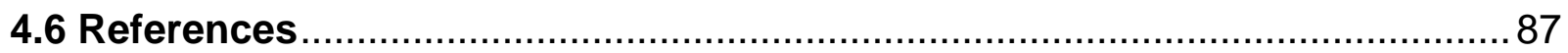

Chapter 5: The Versatility of Bismuth doping through Non-stoichiometric Addition

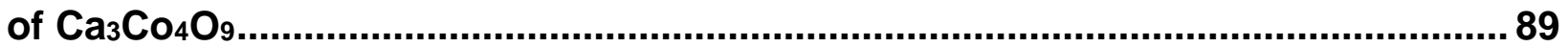

5.1 Background and motivation for non-stoichiometric addition method .......... 89

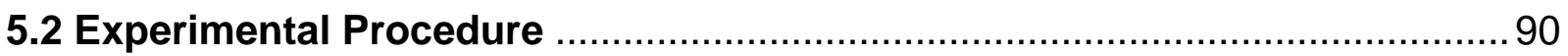

5.3 Simultaneously doping the lattice and grain boundary ............................ 92

5.3.1 Increasing the carrier mobility through bismuth non-stoichiometric addition .. 92

5.3.2 Improvement of grain alignment along with boundary segregation .................94

5.3.3 Significance of Phonon Scattering and a small Mean Free Path (MFP) .......100

5.3.4 Figure of merit of bismuth non-stoichiometric addition doped $\mathrm{Ca}_{3} \mathrm{C}_{4} \mathrm{O}_{9} \ldots . . .102$

5.4 Effects of processing parameters on electrical \& thermal properties......... 103

5.4.1 Electrical performance of $\mathrm{Ca}_{3} \mathrm{Bi}_{3} \mathrm{C}_{4} \mathrm{O}_{9}$ varying ceramic processing parameters. 104

5.5 Summary of results for bismuth non-stoichiometric addition of $\mathrm{Ca}_{3} \mathrm{Co}_{4} \mathrm{O}_{9} 109$ 5.6 References.

Chapter 6: Non-stoichiometric Addition of Barium to Enhance Electrical Power

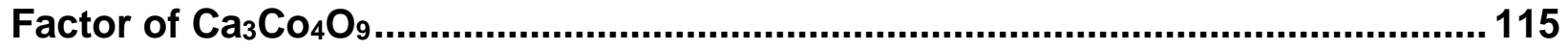

6.1 Background and motivation for Barium as a dopant in $\mathrm{Ca}_{3} \mathrm{Co}_{4} \mathrm{O}_{9} \ldots \ldots \ldots \ldots . . . .115$

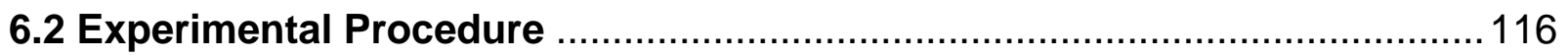

6.3 Effect of barium's grain boundary segregation on the power factor ...........118

6.3.1 Increased electrical properties of $\mathrm{Ca}_{3} \mathrm{Co}_{4} \mathrm{O}_{9}$ through $\mathrm{Ba}$ addition ................118 
6.3.2 Microstructure and nanostructure of Barium doped $\mathrm{Ca}_{3} \mathrm{C}_{4} \mathrm{O}_{9}$......

6.4 Summary of electrical properties of Barium non-stoichiometric addition of

$\mathrm{Ca}_{3} \mathrm{Co}_{4} \mathrm{O}_{9}$

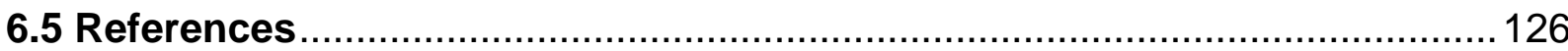

Chapter 7: Non-Stoichiometric dual doping of Bismuth and Barium .................... 127

7.1 Motivation of introducing dual dopants using non-stoichiometric addition

7.2 Experimental Procedure 128

7.3 Finding a balance between carrier mobility \& concentration through tuning dopant concentration

7.4 Summary of results for doping $\mathrm{Ca}_{3} \mathrm{Co}_{4} \mathrm{O}_{9}$ using dual dopants Bismuth \&

Barium through non-stoichiometric addition

7.5 References

Chapter 8: Dual Doping $\mathrm{Ca}_{3} \mathrm{Co}_{4} \mathrm{O}_{9}$ through Cationic Substitution \& NonStoichiometric Addition

8.1 Background and motivation for dual doping with cation substitution and non-stoichiometric addition

8.2 Experimental Procedure 140

8.3 Comparison of Non-stoichiometric dual doping to Cation Substitution \& Non-stoichiometric dual doping

8.4 Summary of results for cation substitution \& non-stoichiometric addition dual doping of $\mathrm{Ca}_{3} \mathrm{Co}_{4} \mathrm{O}_{9}$

8.5 References 148

Chapter 9: Optimization of Dual Doping $\mathrm{Ca}_{3} \mathrm{CO}_{4} \mathrm{O}_{9}$ by Controlling Grain Growth \& Texture while Enhancing Absolute Seebeck Coefficient 150

9.1 Motivation 150

9.2 Experimental Procedure 151

9.3 Decreased electrical resistivity and enhanced thermoelectric properties of $\mathrm{Ca}_{3} \mathrm{Co}_{4} \mathrm{O}_{9}$ induced by dual doping 153

9.3.1 Low electrical resistivity achieved through dual doping 153

9.3.2 Fine tuning eletrical resistivity and Seebeck coefficient through dual dopants.

9.4 Microstructure of dual doped Bismuth \& Barium 154

9.4.1 Structure and lattice parameters 157

9.4.2 Texture and Grain Alignment 157 159 
9.5 Nanostructure analysis of dual doped Bismuth \& Barium through use of TEM

9.6 Calculating the thermal conductivities and $\mathrm{ZT}$ of dual doped $\mathrm{Ca}_{3} \mathrm{Co}_{4} \mathrm{O}_{9} \ldots .164$ 9.7 Summary of results for $\mathrm{Ca}_{3} \mathrm{Co}_{4} \mathrm{O}_{9}$ dual doping of bismuth cation substitution $\&$ barium non-stoichiometric addition. 166

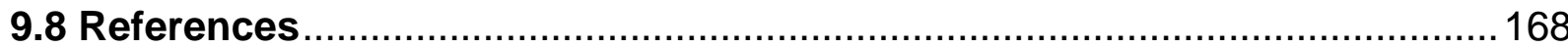

Chapter 10: Conclusion and Suggestions for Future Work ................................170

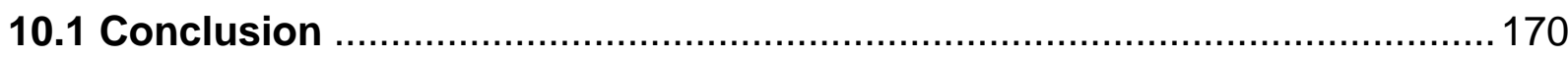

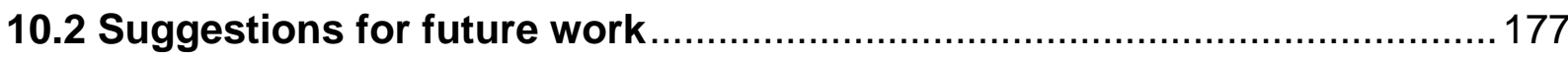

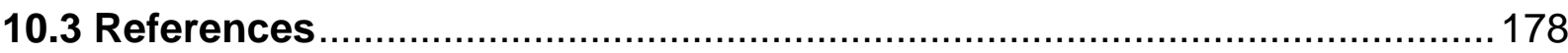

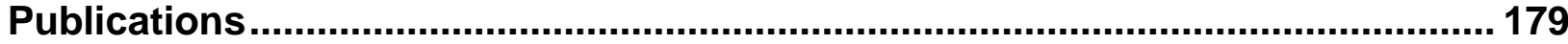




\section{List of Figures}

Figure 1 Cutaway of Thermoelectric Generator.......................................................... 14

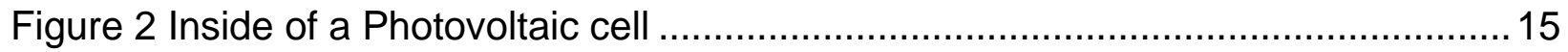

Figure 3 Schematic of thermoelectric device........................................................ 17

Figure 4 P- \& n- type legs of a Thermoelectric Generator.............................................. 18

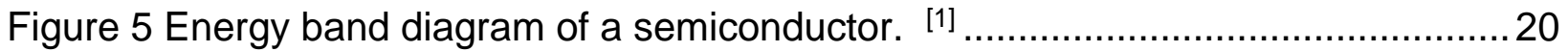

Figure 6 ZT for various p-type thermoelectric materials. ........................................... 22

Figure 7 Typical waste heat and operating temperature ranges of various thermoelectric

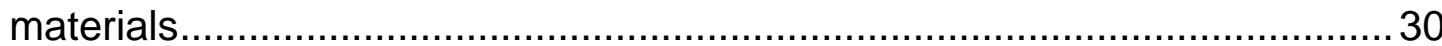

Figure $8 \mathrm{Log}$ electrical conductivity for $\mathrm{Ca}_{3} \mathrm{C}_{4} \mathrm{O}_{9}$ with various dopants .........................32

Figure 9 Absolute Seebeck coefficient for $\mathrm{Ca}_{3} \mathrm{C}_{4} \mathrm{O}_{9}$ with various dopants....................33

Figure 10 Log thermal conductivities of $\mathrm{Ca}_{3} \mathrm{C}_{4} \mathrm{O}_{9}$ with various dopants....................... 33

Figure 11 Figure of merit for $\mathrm{Ca}_{3} \mathrm{C}_{4} \mathrm{O}_{9}$ with various dopants. ..................................... 34

Figure 12 Schematic of alternating subsystems of $\mathrm{Ca}_{3} \mathrm{C}_{4} \mathrm{O}_{9}$ crystal structure .............. 35

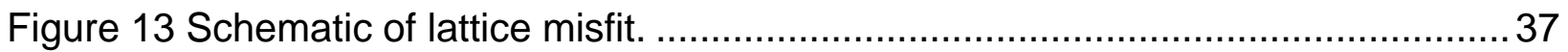

Figure 14 Dimensionless figure of merit vs. temperature of typical current thermoelectric

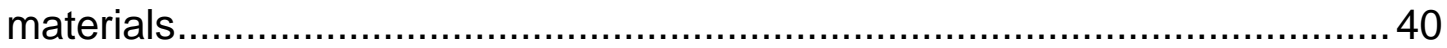

Figure 15 Temperature dependence of electrical resistivity for $\mathrm{Ca}_{3} \mathrm{Co}_{4} \mathrm{O}_{9} \mathrm{Bi}_{2} \mathrm{Te}_{3}$

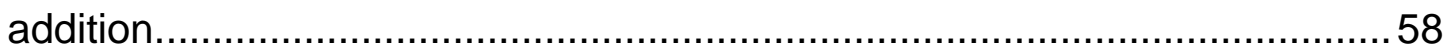

Figure 16 Temperature dependence of absolute seebeck coefficient for $\mathrm{Ca}_{3} \mathrm{Co}_{4} \mathrm{O}_{9}$

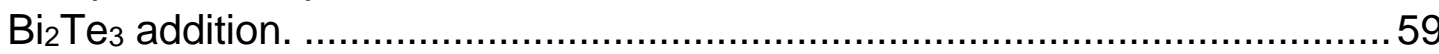

Figure 17 Temperature dependence of power factor for $\mathrm{Ca}_{3} \mathrm{Co}_{4} \mathrm{O}_{9} \mathrm{Bi}_{2} \mathrm{Te}_{3}$ addition...... 60

Figure 18 Temperature dependence of figure of merit for $\mathrm{Ca}_{3-\times} \mathrm{Bi}_{x} \mathrm{Co}_{4} \mathrm{O}_{9+\delta}(\mathrm{x}=0.0$ and

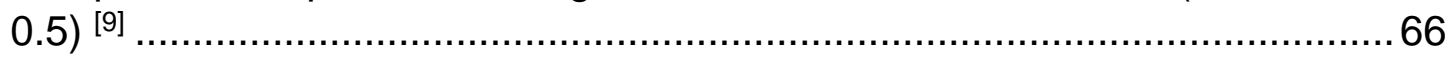

Figure 19 Lattice parameters calculated by Rietveld analysis as a function of $\mathrm{Bi}$ composition $b_{1}$ and $b_{2}$ correspond to the $b$ parameters of $\left[\mathrm{Ca}_{2} \mathrm{CoO}_{3}\right]$ and $\left[\mathrm{CoO}_{2}\right]$ layers, respectively. ${ }^{[11]}$

Figure 20 Temperature dependence of electrical resistivity for $\mathrm{Ca}_{3-\times} \mathrm{Bi}_{x} \mathrm{C}_{4} \mathrm{O}_{9}$ bismuth

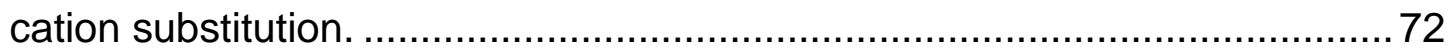

Figure 21 Temperature dependence of absolute seebeck coefficient for $\mathrm{Ca}_{3-\mathrm{x}} \mathrm{Bi}_{\times} \mathrm{C}_{4} \mathrm{O}_{9}$ bismuth cation substitution.

Figure 22 Temperature dependence of power factor for $\mathrm{Ca}_{3-\times} \mathrm{Bi}_{x} \mathrm{CO}_{4} \mathrm{O}_{9}$ bismuth cation substitution. 
Figure 23 SEM cross-sectional view of undoped $\mathrm{Ca}_{3} \mathrm{Co}_{4} \mathrm{O}_{9}$ and doped $\mathrm{Ca}_{3-\mathrm{x}} \mathrm{Bi}_{\times} \mathrm{C}_{4} \mathrm{O}_{9}$

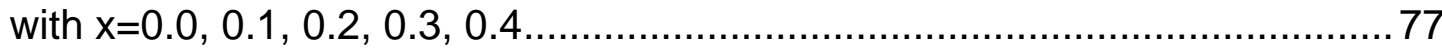

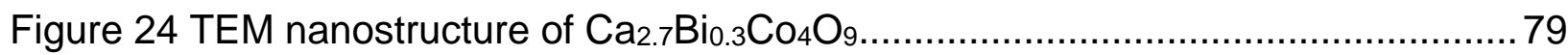

Figure 25 TEM images of undoped and bismuth doped $\mathrm{Ca}_{3} \mathrm{Co}_{4} \mathrm{O}_{9} \ldots \ldots \ldots \ldots \ldots \ldots \ldots \ldots \ldots . . . . . . . . . . . . . . . .80$

Figure 26 Temperature dependence for thermal conductivity of $\mathrm{Ca}_{3-x} \mathrm{Bi}_{\times} \mathrm{C}_{4} \mathrm{O}_{9}$ bismuth

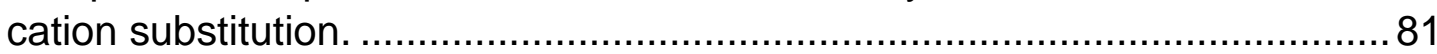

Figure 27 Temperature dependence of figure of merit for $\mathrm{Ca}_{3-\times} \mathrm{Bi}_{\times} \mathrm{Co}_{4} \mathrm{O}_{9}$ bismuth cation substitution.

Figure 28 Temperature dependence of the $\mathrm{ZT}$ values for undoped $\mathrm{Ca}_{3} \mathrm{CO}_{4} \mathrm{O}_{9}$ and

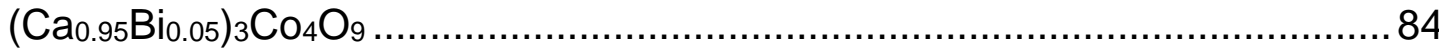

Figure 29 Microstructure drawing of undoped $\mathrm{Ca}_{3} \mathrm{C}_{4} \mathrm{O}_{9}$ and $\mathrm{Ca}_{2.7} \mathrm{Bi}_{0 .} \mathrm{CO}_{4} \mathrm{O}_{9}$. 85

Figure 30 Temperature dependence of electrical resistivity for $\mathrm{Ca}_{3} \mathrm{Bi}_{4} \mathrm{Co}_{4} \mathrm{O}_{9}$ bismuth non-stoichiometric addition.

Figure 31 SEM cross-sectional view of undoped $\mathrm{Ca}_{3} \mathrm{C}_{4} \mathrm{O}_{9}$ and doped $\mathrm{Ca}_{3} \mathrm{Bi}_{4} \mathrm{Co}_{4} \mathrm{O}_{9}$ with $\mathrm{u}=0.0,0.1,0.2,0.3$, and 0.35 .

Figure 32 Temperature dependence of absolute seebeck coefficient for $\mathrm{Ca}_{3} \mathrm{Bi}_{4} \mathrm{Co}_{4} \mathrm{O}_{9}$ bismuth non-stoichiometric addition. .......................................................... 95

Figure 33 X-Ray Diffraction patterns of undoped and doped $\mathrm{Ca}_{3} \mathrm{C}_{4} \mathrm{O}_{9} \ldots \ldots \ldots . . . . . . . . . . . . . .96$

Figure 34 (004) peak from X-Ray Diffraction patterns of undoped and doped $\mathrm{Ca}_{3} \mathrm{C}_{4} \mathrm{O}_{9}$ powders. 97

Figure 35 (a) Temperature dependence of electrical resistivity and (b) absolute seebeck coefficient of $\mathrm{Ca}_{3} \mathrm{Bi}_{4} \mathrm{CO}_{4} \mathrm{O}_{9}$ bismuth non-stoichiometric addition. 99

Figure 36 Temperature dependence of the power factor for $\mathrm{Ca}_{3} \mathrm{Bi}_{4} \mathrm{Co}_{4} \mathrm{O}_{9}$ bismuth nonstoichiometric addition. 100

Figure 37 Temperature dependence of thermal conductivity for $\mathrm{Ca}_{3} \mathrm{Bi}_{4} \mathrm{C}_{4} \mathrm{O}_{9}$ bismuth non-stoichiometric addition. 102

Figure 38 Temperature dependence of figure of merit for $\mathrm{Ca}_{3} \mathrm{Bi}_{4} \mathrm{Co}_{4} \mathrm{O}_{9}$ bismuth nonstoichiometric addition. 103

Figure 39 Temperature dependence of electrical resistivity for $\mathrm{Ca}_{3} \mathrm{Bi}_{0 .} \mathrm{CO}_{4} \mathrm{O}_{9}$ bismuth non-stoichiometric addition with pressing parameter variation. 105

Figure 40 SEM cross-sectional view of undoped $\mathrm{Ca}_{3} \mathrm{CO}_{4} \mathrm{O}_{9}$ and doped $\mathrm{Ca}_{3} \mathrm{Bi}_{4} \mathrm{Co}_{4} \mathrm{O}_{9}$ with $\mathrm{u}=0.0,0.1,0.2,0.3,0.3(0.75 \mathrm{GPa})$, and 0.35 . 106

Figure 41 Temperature dependence of power factor for $\mathrm{Ca}_{3} \mathrm{Bi}_{0.3} \mathrm{Co}_{4} \mathrm{O}_{9}$ bismuth nonstoichiometric addition with pressing parameter variation. 
Figure 42 Temperature dependence of thermal conductivity for $\mathrm{Ca}_{3} \mathrm{Bi}_{0 .} \mathrm{C}_{0} \mathrm{O}_{9}$ bismuth non-stoichiometric addition with pressing parameter variation. 108

Figure 43 Temperature dependence of figure of merit for $\mathrm{Ca}_{3} \mathrm{Bi}_{0.3} \mathrm{C}_{4} \mathrm{O}_{9}$ bismuth nonstoichiometric addition with pressing parameter variation. 109

Figure 44 Microstructure drawing of undoped $\mathrm{Ca}_{3} \mathrm{C}_{4} \mathrm{O}_{9}$ and $\mathrm{Ca}_{2.7} \mathrm{Bi}_{0.3} \mathrm{C}_{4} \mathrm{O}_{9}$. 111

Figure 45 Temperature dependence of electrical resistivity for $\mathrm{Ca}_{3} \mathrm{Ba}_{4} \mathrm{Co}_{4} \mathrm{O}_{9}$ barium non-stoichiometric addition

Figure 46 Temperature dependence of absolute seebeck coefficient for $\mathrm{Ca}_{3} \mathrm{Ba}_{4} \mathrm{Co}_{4} \mathrm{O}_{9}$ barium non-stoichiometric addition.

Figure 47 Temperature dependence of power factor for $\mathrm{Ca}_{3} \mathrm{Ba}_{4} \mathrm{Co}_{4} \mathrm{O}_{9}$ barium nonstoichiometric addition.

Figure 48 SEM cross-sectional view of undoped $\mathrm{Ca}_{3} \mathrm{C}_{4} \mathrm{O}_{9}$ and doped $\mathrm{Ca}_{3} \mathrm{Ba}_{4} \mathrm{Co}_{4} \mathrm{O}_{9}$ with $\mathrm{u}=0.0,0.01,0.05$, and 0.1 .

Figure 49 Diffraction contrast images and the selected area diffraction patterns from the $\mathrm{Ca}_{3} \mathrm{Ba}_{0.05} \mathrm{Co}_{4} \mathrm{O}_{9}$ sample.

Figure 50 Microstructure drawing of undoped $\mathrm{Ca}_{3} \mathrm{C}_{4} \mathrm{O}_{9}$ and $\mathrm{Ca}_{3} \mathrm{Ba}_{0.05} \mathrm{C}_{4} \mathrm{O}_{9} \ldots \ldots \ldots . . . .125$

Figure 51 Temperature dependence of electrical resistivity for $\mathrm{Ca}_{3} \mathrm{Bi}_{4} \mathrm{Ba}_{w} \mathrm{Co}_{4} \mathrm{O}_{9}$ bismuth \& barium non-stoichiometric addition where $\mathrm{u}=0.0,0.02,0.05$, and $0.07 \ldots 132$

Figure 52 Temperature dependence of absolute seebeck coefficient for $\mathrm{Ca}_{3} \mathrm{Bi}_{4} \mathrm{Ba}_{w} \mathrm{C}_{4} \mathrm{O}_{9}$ bismuth \& barium non-stoichiometric addition where $\mathrm{u}=0.0$, $0.02,0.05$, and 0.07 . 133

Figure 53 Temperature dependence of power factor for $\mathrm{Ca}_{3} \mathrm{Bi}_{4} \mathrm{Ba}_{w} \mathrm{Co}_{4} \mathrm{O}_{9}$ bismuth \& barium non-stoichiometric addition where $u=0.0,0.02,0.05$, and $0.07 \ldots . .135$

Figure 54 Temperature dependence of electrical resistivity for $\mathrm{Ca}_{3-x} \mathrm{Bi}_{x} \mathrm{Ba}_{4} \mathrm{C}_{4} \mathrm{O}_{9}$ bismuth cation substitution \& barium non-stoichiometric addition where $u=0.0$, $0.02,0.05$, and 0.07 .

Figure 55 Temperature dependence of absolute seebeck coefficient for $\mathrm{Ca}_{2.8} \mathrm{Bi}_{0.2} \mathrm{Ba}_{4} \mathrm{CO}_{4} \mathrm{O}_{9}$ bismuth cation substitution \& barium non-stoichiometric addition where $u=0.0,0.02,0.05$, and 0.07 .

Figure 56 Temperature dependence of power factor for $\mathrm{Ca}_{2.8} \mathrm{Bi}_{0.2} \mathrm{Ba}_{4} \mathrm{Co}_{4} \mathrm{O}_{9}$ bismuth cation substitution \& barium non-stoichiometric addition where $u=0.0,0.02$, 0.05 , and 0.07 . 146

Figure 57 Temperature dependence of electrical resistivity for $\mathrm{Ca}_{2.9} \mathrm{Bi}_{0.1} \mathrm{Ba}_{4} \mathrm{Co}_{4} \mathrm{O}_{9}$ bismuth cation substitution \& barium non-stoichiometric addition where $\mathrm{u}=0.0$, $0.02,0.05,0.07$, and 0.1 . 154 
Figure 58 Temperature dependence of absolute seebeck coefficient for $\mathrm{Ca}_{2.9} \mathrm{Bi}_{0.1} \mathrm{Ba}_{4} \mathrm{CO}_{4} \mathrm{O}_{9}$ bismuth cation substitution \& barium non-stoichiometric addition where $u=0.0,0.02,0.05,0.07$, and 0.1

Figure 59 Temperature dependence of power factor for $\mathrm{Ca}_{2.9} \mathrm{Bi}_{0.1} \mathrm{Ba}_{4} \mathrm{Co}_{4} \mathrm{O}_{9}$ bismuth cation substitution $\&$ barium non-stoichiometric addition where $u=0.0,0.02$, $0.05,0.07$, and 0.1 . 157

Figure 60 XRD powder diffraction patterns for the $\mathrm{Ca}_{3-x} \mathrm{Bi}_{x} \mathrm{Ba}_{4} \mathrm{Co}_{4} \mathrm{O}_{9}$ samples. 158

Figure 61 SEM images showing crystal texture development of the $\mathrm{Ca}_{3} \mathrm{C}_{4} \mathrm{O}_{9}$ samples with dual element doping of bismuth and barium simultaneously. 160

Figure 62 Microstructure drawing of undoped $\mathrm{Ca}_{3} \mathrm{Co}_{4} \mathrm{O}_{9}$ and $\mathrm{Ca}_{2.9} \mathrm{Bi}_{0 .} \mathrm{Ba}_{0.07} \mathrm{Co}_{4} \mathrm{O}_{9} . .161$

Figure 63 (a) Diffraction contrast image and (b) high resolution TEM from the $\mathrm{Ca}_{2.9} \mathrm{Bi}_{0.1} \mathrm{C}_{4} \mathrm{O}_{9}$ sample. 162

Figure 64 TEM images from the $\mathrm{Ca}_{2 .}{ }_{9} \mathrm{Bi}_{0.1} \mathrm{Ba}_{0.07} \mathrm{C}_{4} \mathrm{O}_{9}$ sample. 163

Figure 65 Temperature dependence of thermal conductivity for $\mathrm{Ca}_{2.9} \mathrm{Bi}_{0.1} \mathrm{Ba}_{4} \mathrm{Co}_{4} \mathrm{O}_{9}$ bismuth cation substitution and barium non-stoichiometric addition where $\mathrm{u}=0.0,0.05$, and 0.07 .

Figure 66 Temperature dependence of figure of merit for $\mathrm{Ca}_{2.9} \mathrm{Bi}_{0.1} \mathrm{Ba}_{u} \mathrm{C}_{4} \mathrm{O}_{9}$ bismuth cation substitution and barium non-stoichiometric addition where $u=0.0,0.05$, and 0.07 . 166

Figure 67 Microstructure development of undoped $\mathrm{Ca}_{3} \mathrm{C}_{4} \mathrm{O}_{9}, \mathrm{Ca}_{2.7} \mathrm{Bi}_{0.3} \mathrm{C}_{4} \mathrm{O}_{9}$, $\mathrm{Ca}_{3} \mathrm{Ba}_{0.05} \mathrm{C}_{4} \mathrm{O}_{9}$, and $\mathrm{Ca}_{2.9} \mathrm{Bi}_{0.1} \mathrm{Ba}_{0.07} \mathrm{CO}_{4} \mathrm{O}_{9}$.

Figure 68 Comparisons of temperature dependence of power factor between a) $\mathrm{Ca}_{2.8} \mathrm{Bi}_{0.2} \mathrm{Co}_{4} \mathrm{O}_{9}$, b) $\mathrm{Ca}_{3} \mathrm{Bi}_{0.3} \mathrm{C}_{4} \mathrm{O}_{9}$, c) $\mathrm{Ca}_{3} \mathrm{Ba}_{0.05} \mathrm{Co}_{4} \mathrm{O}_{9}$, and d) $\mathrm{Ca}_{2.9} \mathrm{Bi}_{0.1} \mathrm{Ba}_{0.07} \mathrm{Co}_{4} \mathrm{O}_{9}$.

Figure 69 Comparisons of temperature dependence of figure of merit between a) $\mathrm{Ca}_{2.8} \mathrm{Bi}_{0.2} \mathrm{Co}_{4} \mathrm{O}_{9}$, b) $\mathrm{Ca}_{3} \mathrm{Bi}_{0.3} \mathrm{Co}_{4} \mathrm{O}_{9}$, c) $\mathrm{Ca}_{3} \mathrm{Ba}_{0.05} \mathrm{Co}_{4} \mathrm{O}_{9}$, and d) $\mathrm{Ca}_{2.9} \mathrm{Bi}_{0.1} \mathrm{Ba}_{0.07} \mathrm{Co}_{4} \mathrm{O}_{9}$. 


\section{List of Tables}

Table 1 EDS spot examination showing the chemistry of segregation regions and neighboring grains in the sample $\mathrm{Ca}_{2 .} \mathrm{Bi}_{0.3} \mathrm{CO}_{4} \mathrm{O}_{9}$.

Table 2 Chemical Compositions of two neighboring grains $A$ (spots 1, 2, 3, and 4) and $B$ (spots $9,10,11$, and 12) and at the grain boundary between (spots 5, 6, 7, and

8) shown in figure 42. 124

Table 3 Lattice parameters for samples with different chemistries.

Table 4 Chemical compositions of two neighboring grains and grain boundary in between.

Table 5 Chemical compositions of two neighboring grains and grain boundary in

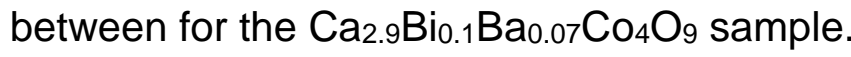




\section{Chapter 1: \\ Background Review of Thermoelectric Generators and Materials}

\subsection{Brief introduction of thermoelectric generators}

Thermoelectric generators (TEGs) are silent devices having no moving parts and are very low maintenance. ${ }^{[1,2]}$ This enables them to be ideal in harsh environments and used in situations where mechanical servicing is not possible or easily accessible. TEGs are also environmentally friendly causing no $\mathrm{CO}_{2}$ emissions and are used to repurpose energy through harnessing waste heat. ${ }^{[1,2]}$ Figure 1 shows a common design and set up of a thermoelectric generator. In 1821 Thomas Seebeck discovered that dissimilar material subjected to different temperatures at opposite ends will produce a voltage. ${ }^{[3,4]}$ This voltage produced from the temperature gradient within became known as the Seebeck Effect. This temperature gradient causes a heat flow within the material creating an electrical potential and diffusing charge carriers throughout the material. ${ }^{[4]}$ It is these charge carriers that are responsible for the current within the material. He then realized that by utilizing this property that heat, waste heat to be more exact, can be "captured" and transformed directly into electrical energy. 


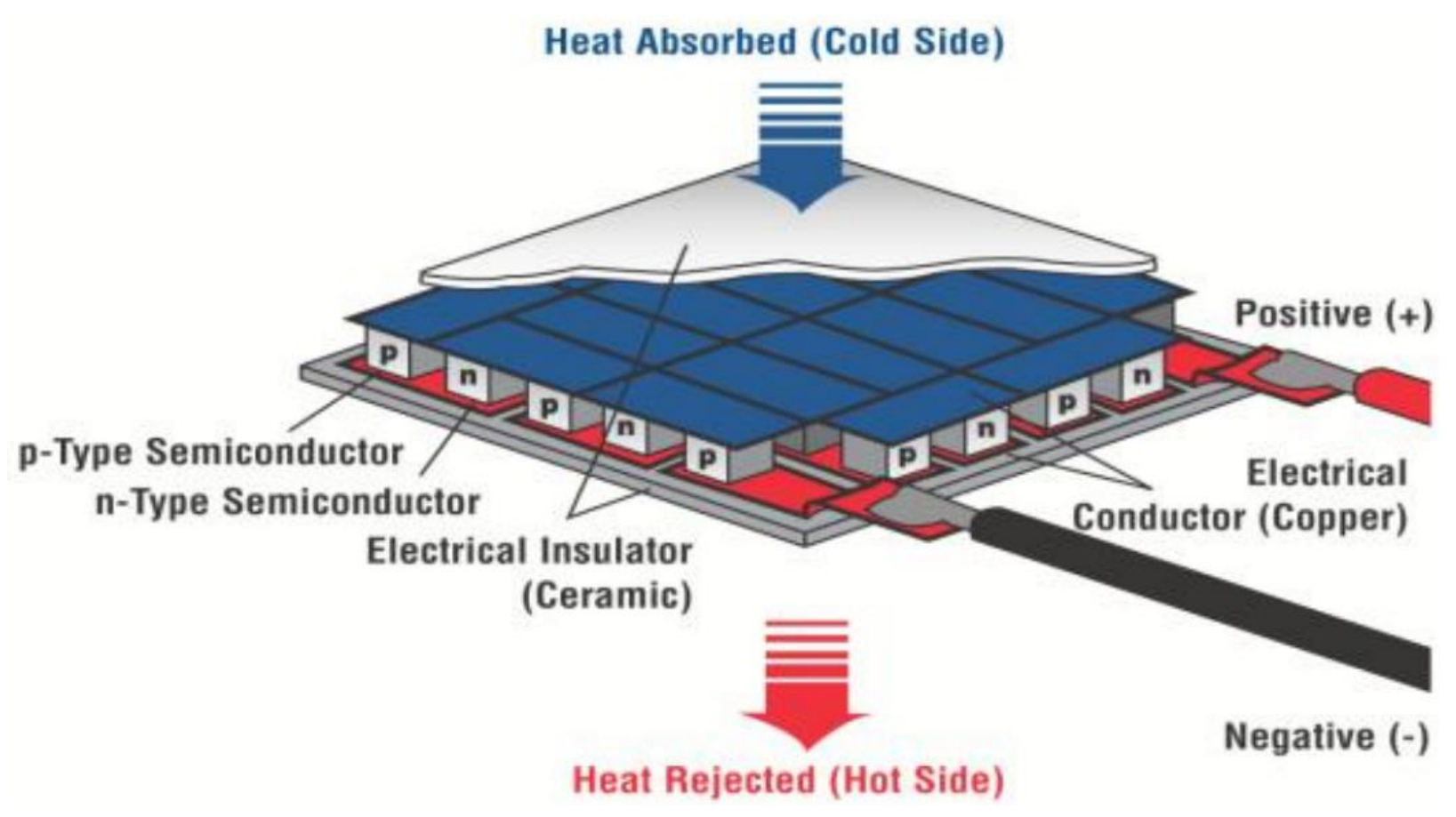

Figure 1 Cutaway of Thermoelectric Generator. [5]

As of now there are two main methods to utilize the Seebeck Effect. The first is through the Photovoltaic process or solar thermal processes. ${ }^{[6]}$ Photovoltaic process shown in figure 2 is the excitement of the electron-hole pair, through light, to create a current within the device. ${ }^{[6,7]}$ This process is commonly seen being used with a flat panel, mainly seen on rooftops of houses, buildings, or in solar farms to harness the light from the Sun. ${ }^{[6]}$ The second is through solar thermal process which utilizes mechanical waste heat through engines. ${ }^{[6,8]}$ In this instance a device can be designed to capture heat from household appliances or heat supplied by means of solar hot-water systems or even from the waste heat of a car engine. More than $60 \%$ of energy is lost, mostly as waste heat, and most mechanical devices operate at a $30 \%$ efficiency, meaning the other $70 \%$ is lost 
during combustion or dissipated into the atmosphere as waste heat as well, causing these all to be optimal methods of harvesting wasted energy. [9,10,11]

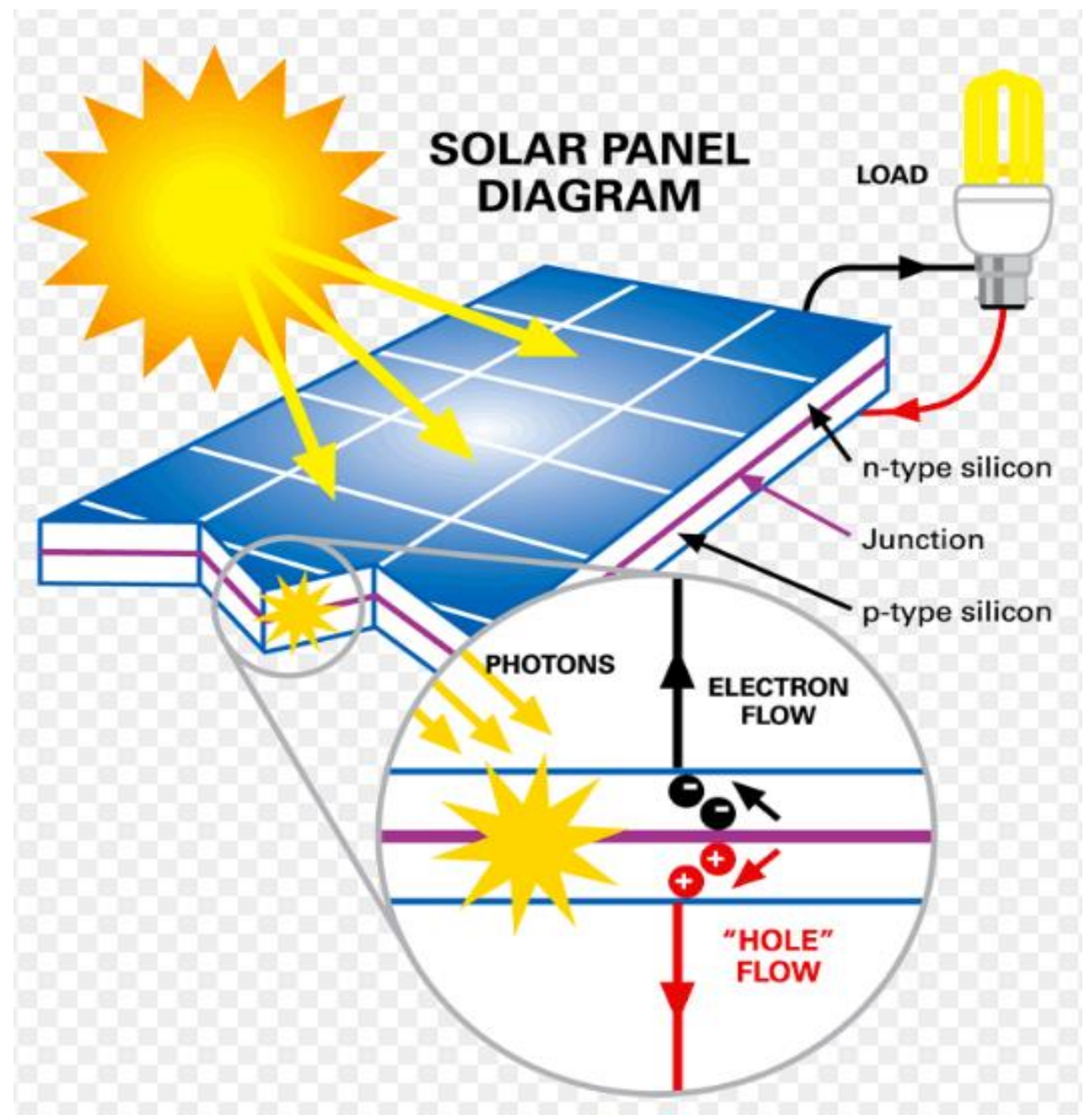

Figure 2 Inside of a Photovoltaic cell [12]

\subsection{P \& $\mathrm{n}$ type of thermoelectric materials}

All circuits are comprised of components with specific charge carriers and just as batteries have a positive and a negative side, so too do TEGs. The charge carriers are the electron-hole pair and the charge-carrying mediums are the negative and positive materials of the device, referred to as the n-type and p-type, respectively. These 
alternating $p$ - and n-type 'legs' of TEGs are shown magnified in figure 3. Although a unicouple is limited by a small voltage generation and is not practical for commercial use, it can be used to further elucidate the diffusion of charge carriers throughout the $p$ - and n-type mediums. How the electron-hole pair diffuse through the system can be seen though the illustration in figure 4. The electron is excited due to the electrical potential created by the thermal gradient and moves to a higher energy level leaving behind a void, or "hole". As the electron continues this trend it moves along the n-type material, since an electron carries a negative charge. The hole, which carries a positive charge, moves in the opposite direction along the $\mathrm{p}$-type material. The $\mathrm{p}$ - and n-types are connected to form a module, via a metal conductor to complete the circuit. ${ }^{[13]}$ They are connected electrically in series to increase its voltage capabilities and thermally in parallel to increase its current capacity. The circuit then sits on a substrate usually made of ceramic or glass. Below is a schematic of a TEG. 


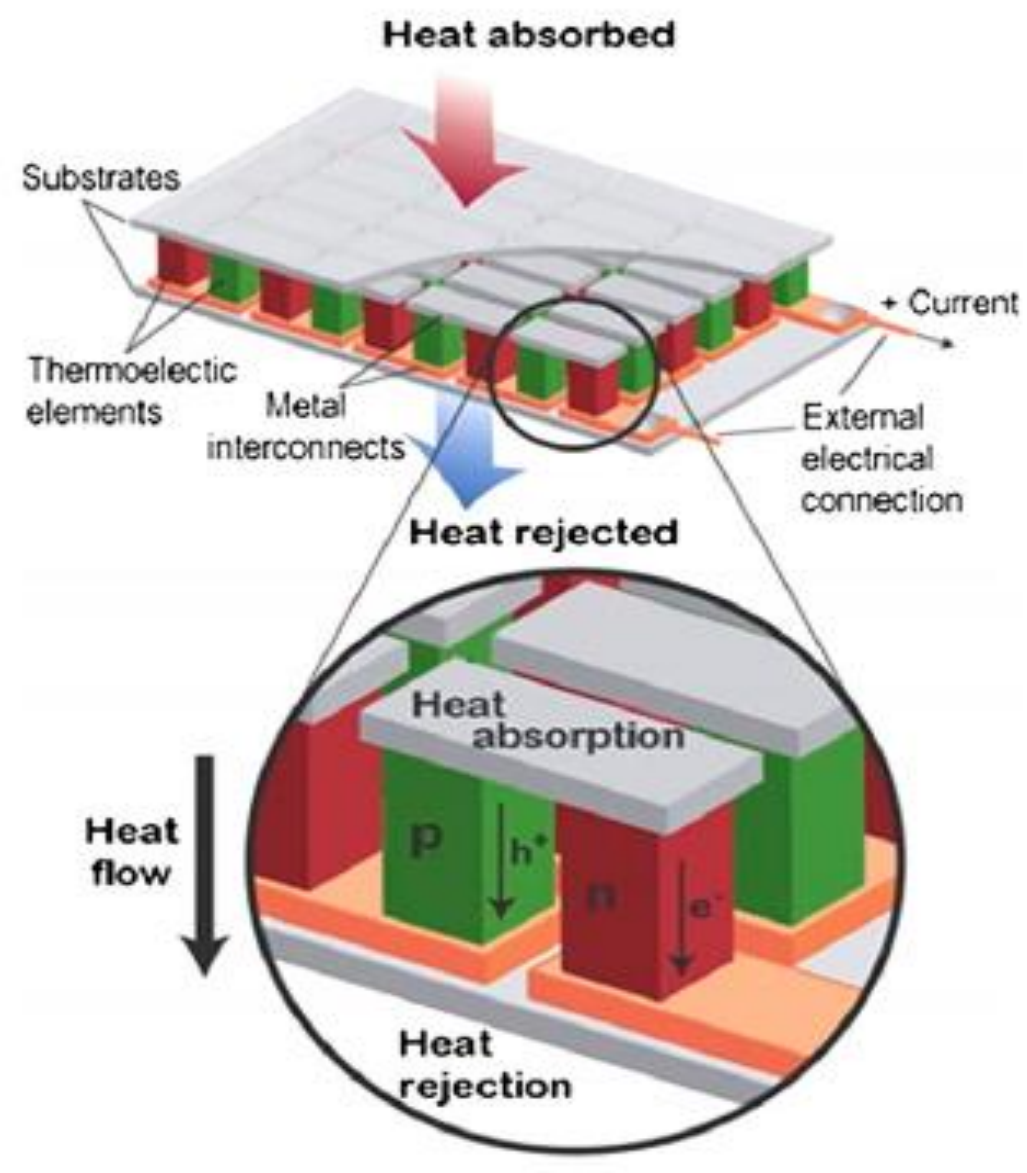

Figure 3 Schematic of thermoelectric device. ${ }^{[14]}$

A semiconductor has a conductivity of that between an insulator and a metal and is often described by its dominating charge carrier. An intrinsic semiconductor is a pure semiconductor uninfluenced by external factors. Its number of charge carriers is strictly determined by the properties of the material itself. When an intrinsic semiconductor is doped with an impurity it takes on extrinsic properties pertaining to the specific dopant thus making it an extrinsic semiconductor. ${ }^{[15,16]}$ This allows the electron or hole to be the dominating carrier thus distinguishing between the $p$ - and $n$-type. ${ }^{[10,11]}$ The Seebeck coefficient is another distinguishing factor in deciding whether the material is $p$ - or n-type. 
A p-type material will have a positive Seebeck coefficient just as an n-type material would have a negative Seebeck coefficient. Figure 4 illustrates how the electron-hole pair move through their respective materials.

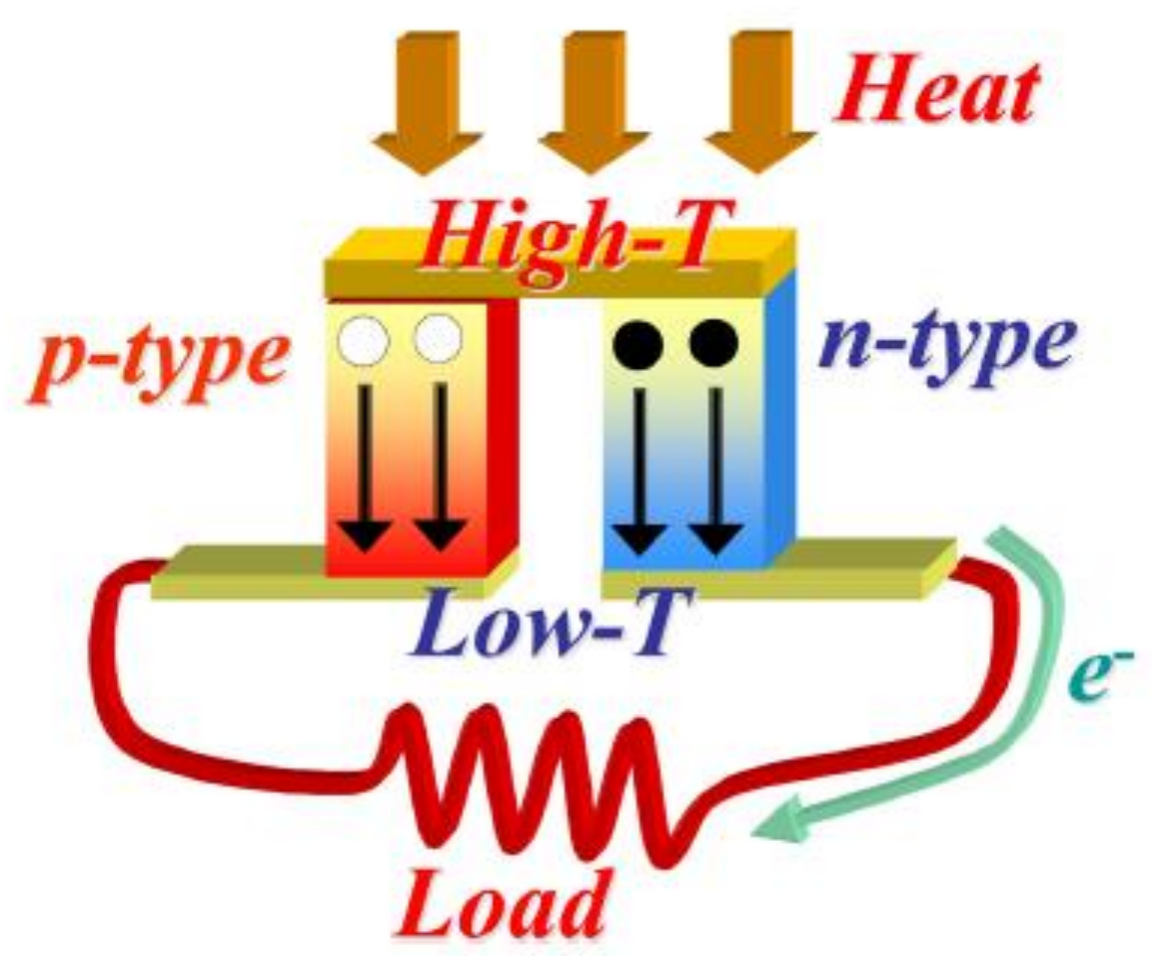

Figure 4 P- \& n-type legs of a Thermoelectric Generator. ${ }^{[4]}$

The two main kinds of dopants are donors and acceptors. Doping a semiconductor has various effects on the material's properties such as indirectly affecting its electrical properties by directly affecting its carrier concentration. [17,18Error! Bookmark not defined.] Doping allows for energy states within the band gap, which is the gap between the conductive band and the valence band shown in figure 5. ${ }^{[18]}$ Depending on the dopant type these energy states can be close to its corresponding energy band. [Error! Bookmark not defined.] Donor dopants will create energy states close to the conduction band, while acceptor 
dopants will create energy states close to the valence band. [Error! Bookmark not defined.] This gap that is created between the energy state and its nearest band is referred to as the dopant site. [Error! Bookmark not defined.]

A donor dopant has extra valence electrons so that when it is introduced as an impurity it will increase the amount of electrons in the conductor and in turn increase its overall negative charge. This causes the electron to be the majority charge carrier making it a negative material or an n-type semiconductor. ${ }^{[15,16]}$ An acceptor impurity atom has fewer valence electrons than the atom it's replacing allowing it to accept electrons from the conductor so there will be a decrease in electrons thus decreasing its electrical charge. Decreasing the electrical charge will make it positively charged causing the hole to be the majority charge carrier. ${ }^{[15,16]}$ This component becomes a positive material or a p-type semiconductor. Together, the $n$ - and p-type semiconductors work in tandem to create a circuit for current to pass through.

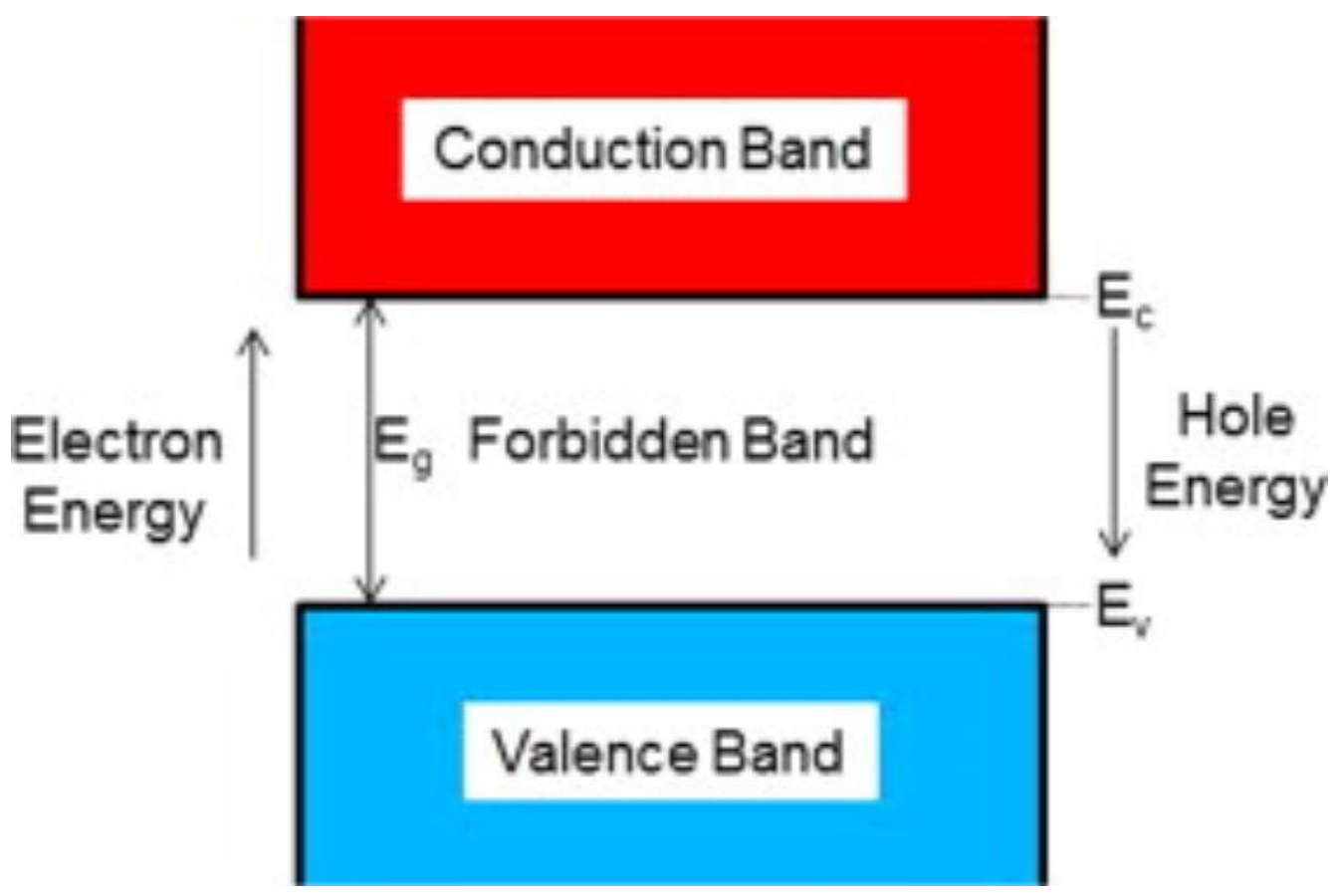


Figure 5 Energy band diagram of a semiconductor. [114]

\subsection{Figure of Merit ZT and specific ZTs of various materials}

TE materials are measured by their figure of merit, ZT. The figure of merit is a dimensionless numerical value that addresses the efficiency of the material. ${ }^{[19]}$ It is the ability of a given material to efficiently produce thermoelectric power. ZT is expressed as;

$$
Z T=\left(\sigma S^{2} / K\right) T
$$

Where $\sigma$ is the electrical conductivity in Siemens per meter $(\mathrm{S} / \mathrm{m}), \mathrm{S}$ is the Seebeck coefficient in microvolts per kelvin $(\mu \mathrm{V} / \mathrm{K}), \mathrm{K}$ is the thermal conductivity in watts per millikelvin (W/mK), and $\mathrm{T}$ is the temperature in Kelvin (K). ${ }^{[20,21,22,23]}$ Each the $p$-type and n-type components have their own figure of merit demonstrating their unique level of efficiency. These are then used as input into another equation, referred to as the effective figure of merit, $Z \bar{T}$. The sole purpose of this equation is to take the figure of merit of each individual $\mathrm{p}$ - and $\mathrm{n}$-type component in to consideration and combine them in to a unified and useful value, expressed;

$$
Z \bar{T}=\frac{\left(S_{p}-S_{n}\right)^{2_{\bar{T}}}}{\left[\left(\rho_{n} \kappa_{n}\right)^{1 / 2}+\left(\rho_{p} \kappa_{p}\right)^{1 / 2}\right]^{2}}
$$

Where the subscripts $p$ and $n$ indicate that they pertain to the $p$-type leg and the $n$-type leg, respectively. Furthermore, $\rho$, which represents the electrical resistivity in micro ohm meters $(\mu \Omega \mathrm{m})$, is the inverse of $\sigma$ and $\bar{T}$ is the average temperature between the hot and cold surfaces measured in Kelvin. ${ }^{[23]}$ This new modified figure of merit containing the 
efficiencies of both the $\mathrm{p}$ and $\mathrm{n}$ types can now be used in an equation discovered in 1909 by Edmund Altenkirch to find the overall maximum efficiency of the device

$$
\eta_{\max }=\frac{T_{H}-T_{C}}{T_{H}} \frac{\sqrt{1+Z \bar{T}}-1}{\sqrt{1+\overline{z T}}+\frac{T_{C}}{T_{H}}}
$$

Where the subscripts $\mathrm{H}$ and $\mathrm{C}$ represent hot and cold temperatures in Kelvin respectively. $[3,23]$ Since the scope of this paper only encompasses the p-type, the main focus will be on equation 1 , the figure of merit.

The above is an explanation of the physical meaning of this dimensionless figure of merit; however, to fully grasp its concept and further demonstrate its importance, the figure of merits of different materials can be compared. This way the figure of merit of a particular material, when discussed, can be put into perspective with respect to other types of materials. As seen in figure 6 , lead, $\mathrm{Pb}$, containing material demonstrates a high figure of merit. The main concerns with this material is that it only produces this efficiency at the mid temperature ranges and lead has a high toxicity.

When analyzing the high temperature ranges there are two standout materials in figure 6. $\mathrm{Yb}_{14} \mathrm{MnSb}_{11}$ has a high figure of merit in the high temperature range but antimony, $\mathrm{Sb}$ is known to be toxic as well making it an undesirable candidate. Silicon germanium, SiGe, is not considered a toxic material; however, it oxidizes at high temperatures necessitating its use in a vacuum environment. These are common issues in existing materials leaving research advances in the high temperature range wide open for materials containing low toxicity at a low cost. To be competitive in the high 
temperature range a material's figure of merit must surpass that of silicon germanium or be competitive at a lower cost and/or toxicity.

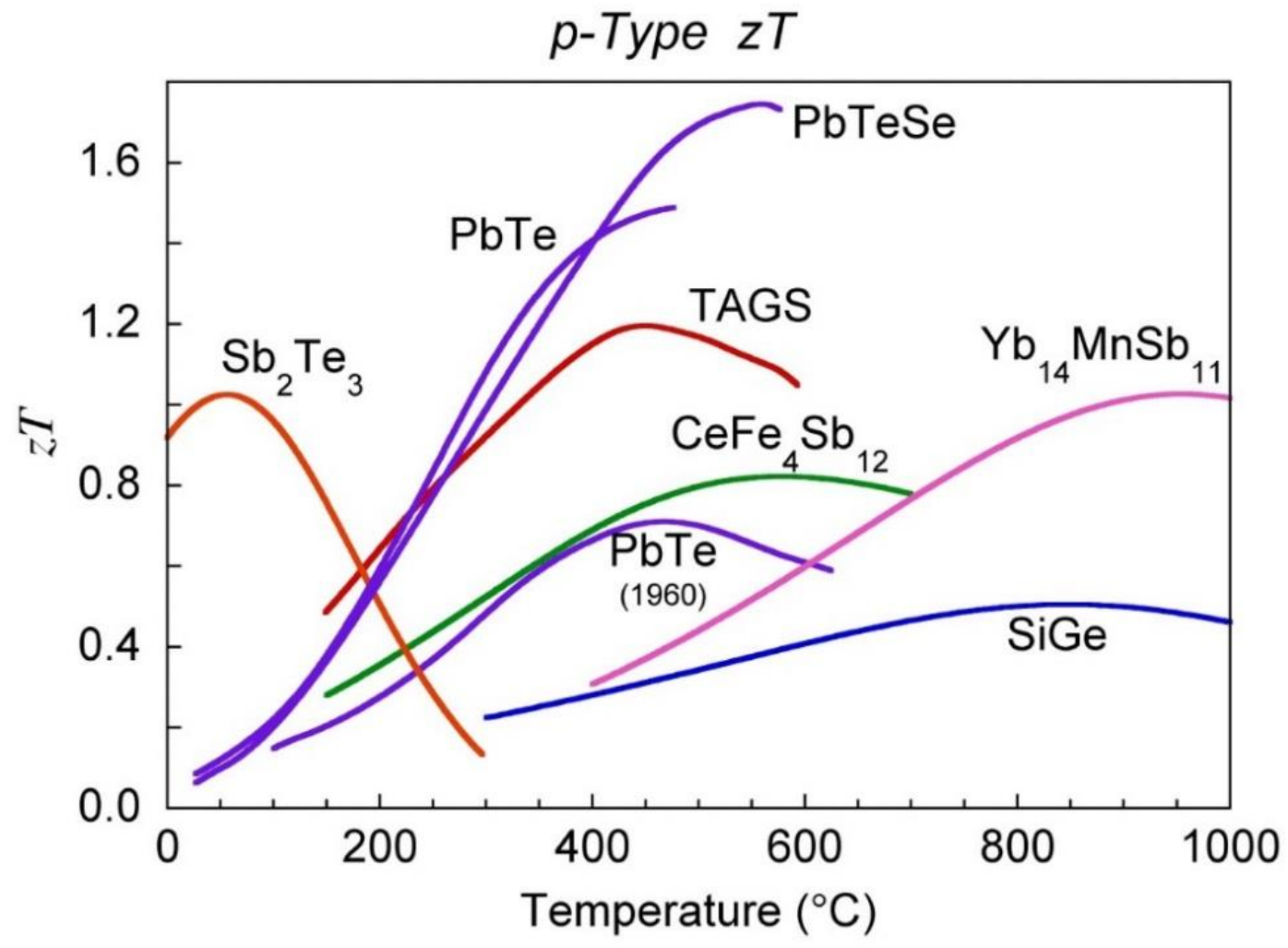

Figure $6 Z T$ for various p-type thermoelectric materials. ${ }^{[24]}$

\subsection{Physical characteristics of thermoelectric materials}

\subsubsection{Electrical Properties of $p$ - \& n- type materials}

To better understand the figure of merit its subcomponents such as the electrical conductivity, Absolute Seebeck Coefficient and the thermal conductivity must be further explained. The electrical conductivity of a material is its measurement or ability to conduct or transfer electricity. A high electrical conductivity means that the component in question will allow electricity to flow more freely through it because it will conduct it not resist it. 
This flow or speed is also referred to as its carrier mobility. ${ }^{[25]}$ The three main factors that affect the conductivity are demonstrated in the equation below

$$
\rho=\frac{1}{\sigma}=\frac{1}{\mu n e}
$$

Where $\rho$ is the electrical resistivity in micro ohm meters $(\mu \Omega m), \sigma$ is the electrical conductivity in Siemens per meter $(\mathrm{S} / \mathrm{m}), \mathrm{mu}, \mu$, is the carrier mobility in centimeters squared per Volt seconds $\left(\mathrm{cm}^{2} / \mathrm{V} \cdot \mathrm{s}\right), \mathrm{n}$ is the carrier concentration in units per centimeter cubed $\left(\mathrm{cm}^{-3}\right)$, and $\mathrm{e}$ is the charge of the carrier. [25] For this reason the electrical conductivity will be referred to in this dissertation as its reciprocal, or the electrical resistivity.

Carrier concentration is the concentration of the dominating charge carrier for that particular material and the carrier mobility is the freedom of flow of electrons throughout. The concentration and mobility are both directly correlated and directly proportional to the electrical conductivity, making them inversely proportional to the resistivity. If either the carrier mobility or carrier concentration decreases, the resistivity increases and vice versa. A high carrier mobility and high carrier concentration lead to good electrical properties. ${ }^{[21]}$ Conceptually a high mobility means the charge carrier in question can move freely causing a flow of current and the higher the concentration the higher the flow of current. Generally speaking, material with a high density will achieve a low electrical resistivity. A high density reflects that of a material with high grain alignment which act as the transport highway for the charge carriers. Material with poor alignment will contain 
more grains oriented more perpendicular to one another thus impeding the electron transport.

The Seebeck coefficient is the proportionality constant between the voltage produced and the difference in temperature across the junction of semiconductors. ${ }^{[3]}$ Platinum probes are used to measure the Seebeck coefficient since they are highly unreactive, and they are resistant to corrosion even at high temperatures. The Relative Seebeck Coefficient of the material is measured, which is the simultaneous influence of the Seebeck Coefficient of the material and the contribution of the Seebeck Coefficient of the platinum probes used to take the measurement. Only the Seebeck Coefficient of the material is desired, referred to as the Absolute Seebeck Coefficient, so the influence of the platinum probe must be subtracted from the relative Seebeck Coefficient. The platinum thermocouple has been studied and accounted for in order to isolate the results of just the material, leaving behind the desired Absolute Seebeck Coefficient.

The predicament with the two electrical properties (electrical resistivity and Absolute Seebeck coefficient) is that they are intimately connected; however, not in a favorable way. If a doping impurity deposits itself at the grain boundary when it is introduced to the baseline it will act as a filter reducing the carrier concentration. This reduction in carrier concentration will enhance the Seebeck coefficient as it will be seen later on. A high carrier concentration is desirable to achieve a low resistivity yet a low carrier concentration is beneficial for achieving a high Seebeck coefficient. To avoid this paradox one can attempt to find a balance with the carrier mobility and carrier concentration. When the carrier concentration is decreased, to benefit the Seebeck coefficient, the carrier mobility can theoretically be increased to preserve the resistivity. 
The idea is to find the balance that optimizes both parameters. This is done mainly through ion doping to create an extrinsic semiconductor. Doping with the proper impurity, in theory, one should be able to alter the material's structure and grain texture to improve upon its properties.

To better demonstrate the electrical transport properties and make them more concise they can be combined to form one factor. This combination, commonly referred to as the power factor, is the materials ability to produce electrical power. ${ }^{[4,23]}$ The power factor is expressed as,

$$
\mathrm{PF}=\mathrm{S}^{2} \sigma
$$

Where $S$ is the Absolute Seebeck Coefficient in microvolts per kelvin $(\mu \mathrm{V} / \mathrm{K})$ and $\sigma$ is the electrical conductivity in Siemens per meter $(\mathrm{S} / \mathrm{m}) .{ }^{[4,23]}$ The higher the power factor the greater the electrical power. Also the Seebeck coefficient is a quadratic, meaning not only does it dominate the outcome of the power factor but it negates the fact that an n-type component has a negative Seebeck coefficient value which will then always give a positive value for the power factor.

The figure of merit can easily be summed up as the division of the electrical transport properties (power factor) by the thermal transport properties (thermal conductivity). In order to achieve a desirable device, a high figure of merit must first be attained. It has already been stated that this can be done by increasing its electrical properties and decreasing its thermal properties. According to the Wiedemann-Franz Law, the electrical conductivity, Absolute Seebeck Coefficient and thermal conductivity are all interconnected. ${ }^{[19]}$ In pursuance of lowering the thermal conductivity the electrical 
properties must suffer and vice versa. It has been shown so far how the electrical conductivity and Absolute Seebeck coefficient are connected. Next is to demonstrate how the thermal properties are intertwined in an adverse way as well.

\subsubsection{Thermal Properties of $p-\& n$ - type materials}

The thermal transport properties are comprised solely of the thermal conductivity making it much more transparent to work with than the electrical properties. A low thermal conductivity is desirable which assures that the device will not dissipate the system's heat but instead act as an insulator by trapping and using the heat. After all, the greater the heat gradient the higher the voltage output of the device. This explains why TE generators generally are not good heat sinks. The thermal conductivity is calculated by the equation

$$
\mathrm{K}=\lambda \mathrm{C}_{\mathrm{p} \rho \mathrm{m}}
$$

Where $K$ is the thermal conductivity in watts per millikelvin $(\mathrm{W} / \mathrm{mK}), \lambda$ is the thermal diffusivity in meters squared per seconds $\left(\mathrm{m}^{2} / \mathrm{s}\right), \mathrm{C}_{\mathrm{p}}$ is the specific heat in joules per kilogram Kelvin $(\mathrm{J} / \mathrm{kg} \cdot \mathrm{K})$, and $\rho_{m}$ is the mass density in grams per centimeter cubed $\left(\mathrm{g} / \mathrm{cm}^{3}\right) \cdot{ }^{[6]}$

Despite being more concise than the electrical transport properties, the thermal conductivity is still a very difficult component to fine tune, in some ways perhaps more difficult. Just as the electrical resistivity can be broken into the carrier concentration and the carrier mobility, the thermal conductivity, $\mathrm{K}$, too can be broken down into two components shown by equation 7 .

$$
\mathrm{K}=\mathrm{K}_{\mathrm{e}}+\mathrm{K}_{\mathrm{L}}
$$


Where $\mathrm{K}_{\mathrm{e}}$ represents the electronic thermal conductivity and $\mathrm{K} \mathrm{L}$ represents the lattice or phonon thermal conductivity. ${ }^{[26,27,28]}$ According to the Wiedemann-Franz Law the electrical and thermal conductivities are intimately intertwined through the electronic thermal conductivity as shown below.

$$
K_{e}=L \sigma T
$$

Where $\mathrm{K}_{\mathrm{e}}$ is the electronic thermal conductivity, $\mathrm{L}$ is the Lorentz number $\left(2.44 \times 10^{-8} \mathrm{~V}^{2} / \mathrm{K}^{2}\right.$ for free electrons), $\sigma$ the electrical conductivity and $T$ is the temperature in Kelvin. ${ }^{[26,27,28]}$ To be more exact, the electrical and thermal conductivities are directly correlated to each other, through the electrical conductivity, as can be seen in equation 8 . When the electrical properties are improved, the thermal properties suffer which proves to be problematic in the overall enhancement of the figure of merit, which is the overarching goal.

It was later studied and found that when the electrical conductivity, $\sigma$, increases with temperature, only the electronic thermal conductivity component, $\mathrm{K}_{\mathrm{e}}$, increases shown in equation 8 and the phonon thermal conductivity, $\mathrm{KL}_{\mathrm{L}}$, is left unaffected. ${ }^{[29,30]}$ It was then discovered that the ratio of $\mathrm{K}_{\mathrm{e}} / \mathrm{K}_{\mathrm{L}}$ is very small so it can be assumed that the phonon thermal conductivity is much greater than the electronic thermal conductivity and thus is the dominant force in determining the overall thermal conductivity. ${ }^{[29,30]}$ This allows the lack of control over the increase in the electronic thermal conductivity to be of little concern and focus can be moved to reducing the lattice thermal conductivity in order to increase efficiency. 
Phonons play a crucial role in the electrical and thermal properties of thermoelectric material. The p-type and n-type components are comprised of atoms that are bound together in a three dimensional pattern forming what is called a crystal lattice. $[29,31]$ The atoms in the lattice act as if they are attached by springs thus vibrating due to any external energy that may be expelled into the system. ${ }^{[29,31]}$ In this case the energy would be that of the waste heat which also creates the heat gradient to excite the atoms. These vibrations create mechanical waves containing a specific energy and momentum. $[29,31]$ These wave "packets" of energy are treated as particles and are referred to as phonons. Just as Einstein's quanta or packets of light are referred to photons, packets of lattice vibrational energy are referred to as phonons. Phonons and electrons are the main excitations in solids. ${ }^{[32]}$

One way to decrease the lattice thermal conductivity is by enhancing phonon scattering. ${ }^{[20,29,30]}$ A common method used is to introduce heavy elements as dopants to aid in this scattering. [33] Decreasing the thermal conductivity can also be done by decreasing the grain size, thus having an increase in the boundary density. This increase in boundary density will act as a filter and be responsible for controlling the carrier concentration. Also, smaller grain size means more boundaries and a shorter mean-free path (MFP). ${ }^{[29,30]}$ The mean-free path is the path the phonon follows before its next scattering event and the mean-free time is the amount of time until the next scattering event. ${ }^{[29,30]}$ The shorter the mean free path and mean free time are the more scattering occurs and the lower the phonon thermal conductivity; the driving component of the overall thermal conductivity. 
In the mid 1990's a concept called "phonon glass electron crystal" was proposed which decoupled the two components of the thermal conductivity allowing for a low thermal conductivity, due to phonon scattering as in a glass, and a high electric conductivity, due to increased electron mobility as in a crystal. ${ }^{[3,19]}$ This way the electrical properties can be improved upon without negatively effecting the thermal properties. As of present day scientists have not yet found a perfect replica of the phonon glass electron crystal scenario. [19]

\subsection{Oxides and Calcium Cobaltite as Thermoelectric materials}

\subsubsection{Use of oxides as Thermoelectric Materials}

Many approaches have been made to maximize the efficiency of the Thermoelectric Generator. Although the design of the circuit can be altered, most hope lies within doping the chemistries of the $\mathrm{p}$-type and n-type material with impurities in order to improve their electrical and thermal properties. Common thermoelectric materials used today are doped alloy semiconductors such as $\mathrm{Bi}_{2} \mathrm{Te}_{3}, \mathrm{PbTe}$ and $\mathrm{SiGe}$. ${ }^{[25]}$ These materials have poor durability at high temperatures, tend to oxidize and are unstable due to decomposition. ${ }^{[34]}$ These alloy semiconductors also seem to face the complication of melting at high temperatures along with having a high toxicity and are costly. ${ }^{[34]}$ These characteristics are detailed in figure 7 . These issues are unfavorable and have been the cause for a search of better and more efficient materials. 


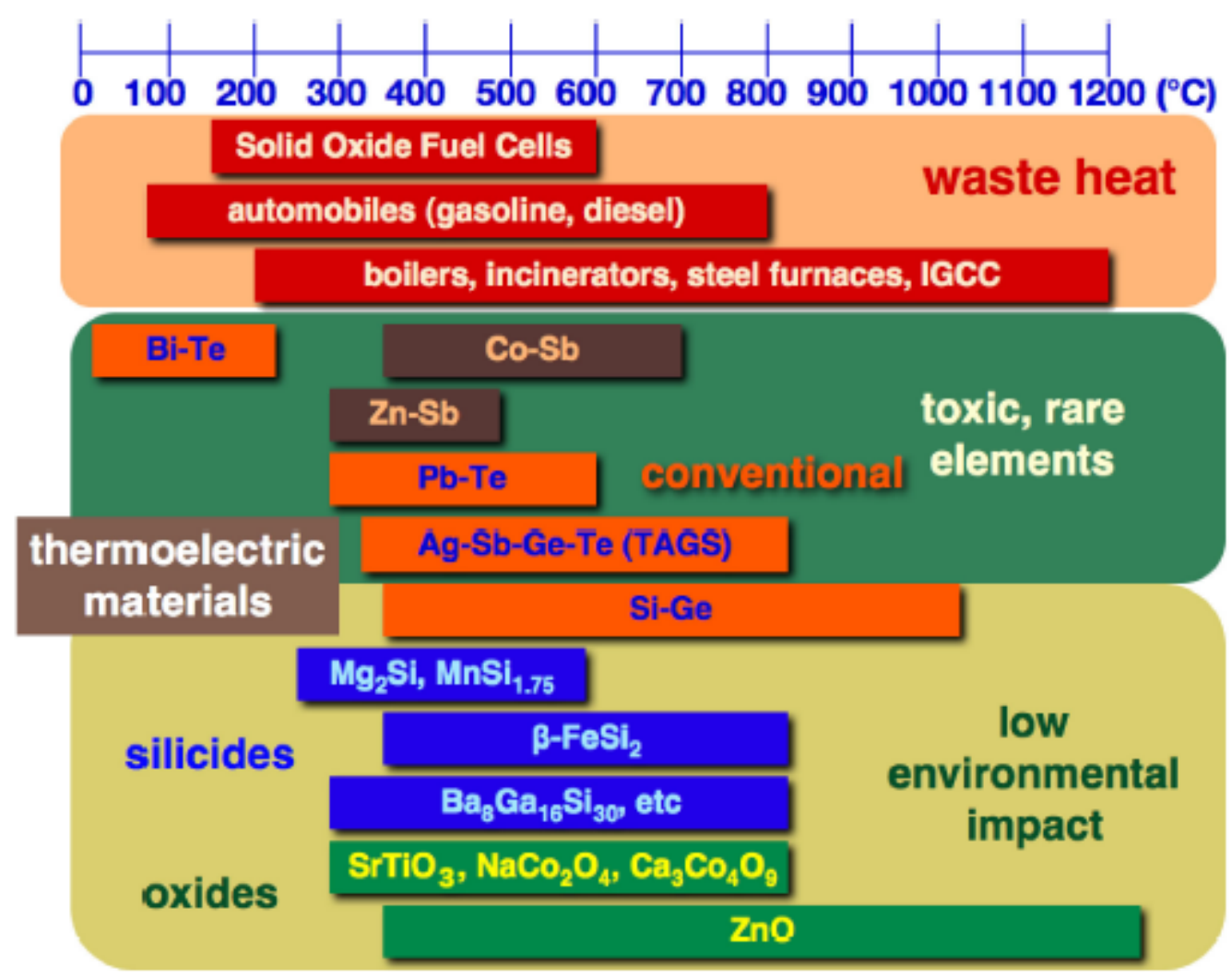

Figure 7 Typical waste heat and operating temperature ranges of various thermoelectric materials. ${ }^{[4]}$

Oxides were first overlooked as thermoelectric material due to the belief of their having low figure of merits caused by poor carrier mobility from their ionic structures. ${ }^{[30,35]}$ However, the discovery of $\mathrm{Na}_{\times} \mathrm{Co}_{2} \mathrm{O}_{4}$ in 1997 with a high Seebeck coefficient of around $100 \mu \mathrm{V} / \mathrm{K}$ at room temperature dismissed the conventional wisdom and ignited extensive research into layered cobalt oxides. ${ }^{[15]}$ Since oxides have high thermal and chemical stability they present a much more promising alternative than alloy semiconductors. Oxides are also easier to handle in the lab because of their nontoxicity and high oxide resistance which can also be seen from figure 7 . These attributes helped to push oxides into the forefront of research. 
$\mathrm{Na}_{x} \mathrm{Co}_{2} \mathrm{O}_{4}$ is just one of many oxides that have been studied and are being used in labs today. $\mathrm{Ca}_{3} \mathrm{Co}_{4} \mathrm{O}_{9}$ is another $\mathrm{Co}$-based oxide that has a thermoelectric performance of its single crystal comparable to that of alloys allowing it to be a promising candidate for research. ${ }^{[34]} \mathrm{Ca}_{3} \mathrm{C}_{4} \mathrm{O}_{9}$ has been studied extensively in the past years and it has been found that the performance of the single crystal shows great promise as a thermoelectric material. The figure of merit of the $\mathrm{Ca}_{3} \mathrm{C}_{4} \mathrm{O}_{9}$ single crystal is $\sim 0.87$ at $973 \mathrm{~K}$ but the polycrystalline bulk sample, with its highest ZT reported as 0.22 at $1173 \mathrm{~K}$ for pure $\mathrm{Ca}_{3} \mathrm{Co}_{4} \mathrm{O}_{9}$ and 0.32 at $1000 \mathrm{~K}$ for bismuth and sodium substitution, still needs improvement and is not yet suitable for technical or commercial application. ${ }^{[36,37,38]}$ This is mainly due to an intrinsically low electrical conductivity and a high thermal conductivity. [36]

Many attempts have been made to improve upon the thermoelectric performance of this already promising material. Some solutions involve ion doping or altering the fabrication process. Most hopes, however, are to decrease the thermal conductivity by means of doping through cationic substitution at the $\mathrm{Ca}$ site with $\mathrm{Sr}, \mathrm{Y}, \mathrm{Bi}$ and other rareearth elements such as $\mathrm{Yb}, \mathrm{Lu}, \mathrm{Dy}, \mathrm{Er}, \mathrm{Ho}$, and Eu which are detailed in figures 8-11. [4,27] There are also reports of doping at the Co site which are included in figures 8-11 as well; however, the Ca site has proven to be more beneficial and is what this dissertation will focus on. The following four graphs were published by Jeffrey Fergus in Oxide materials for high temperature thermoelectric energy conversion and detail the results of various dopants in the $\mathrm{Ca}_{3} \mathrm{Co}_{4} \mathrm{O}_{9}$ ceramic and under differing processing parameters. VPP represents the data from $\mathrm{Ca}_{3} \mathrm{C}_{4} \mathrm{O}_{9}$ ceramics with varied processing parameters under undoped conditions. 
Figure 8 shows the log electrical conductivity in $\mathrm{S} / \mathrm{m}$ and this range would be equivalent to an electrical conductivity of the magnitude $10^{4} \mathrm{~S} / \mathrm{m}$ or an electrical resistivity in the range of $100 \mu \Omega \mathrm{m}$. Figure 9 shows the thermal power or Seebeck coefficient of these same materials which range within a window of $100-150 \mu \mathrm{V} / \mathrm{K}$ depending on the material or processing parameters used. Figure 10 is the log thermal conductivities which would equate to around $2 \mathrm{~W} / \mathrm{mK}$ and figure 11 shows the figure of merits for these materials. It is evident from figure 11 that none of these materials have been reported as passing the $0.5 \mathrm{ZT}$ mark. Although these specific materials are the better alternative to alloy semi-conductors and skutterudites, in regards to their thermal and chemical stability, along with low toxicity and cost, their ZT values are still inferior.

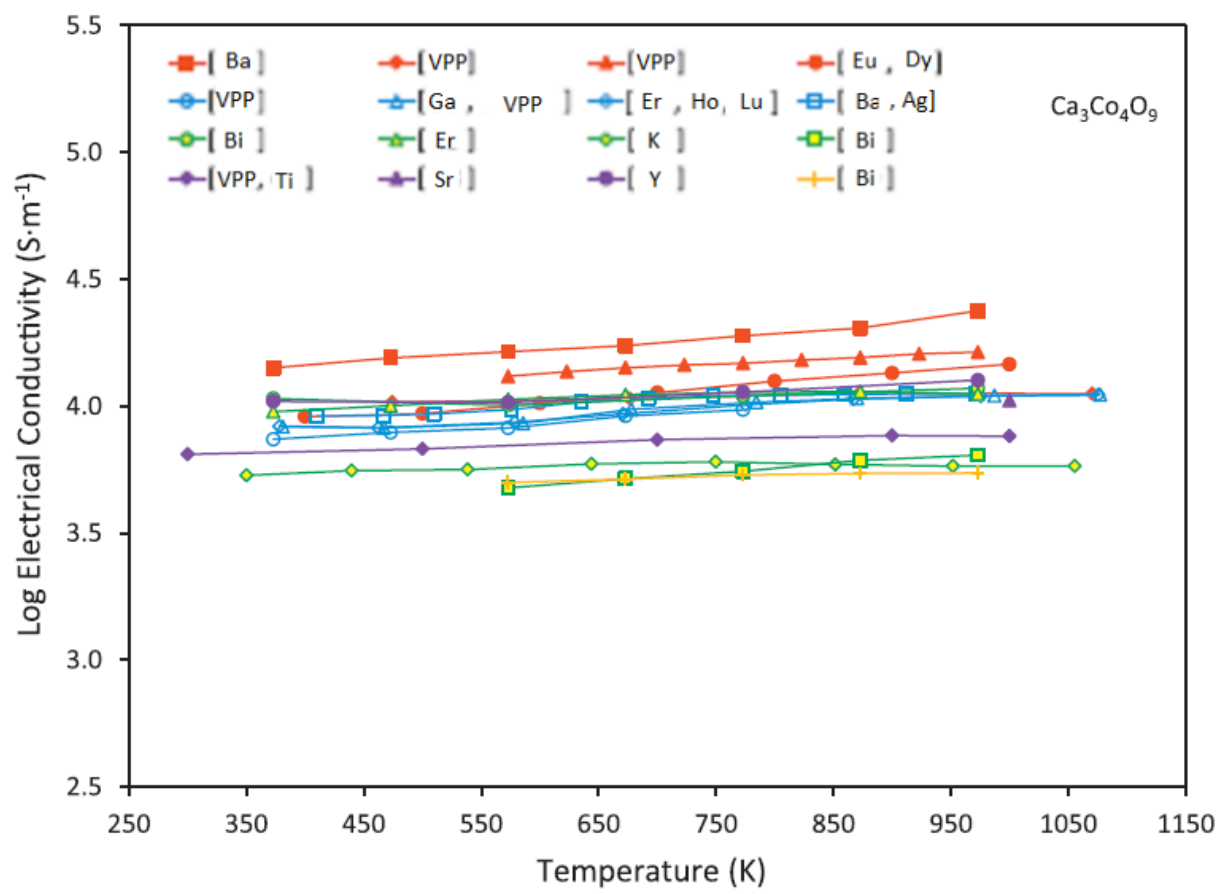

Figure 8 Log electrical conductivity for $\mathrm{Ca}_{3} \mathrm{Co}_{4} \mathrm{O}_{9}$ with various dopants. [39] 


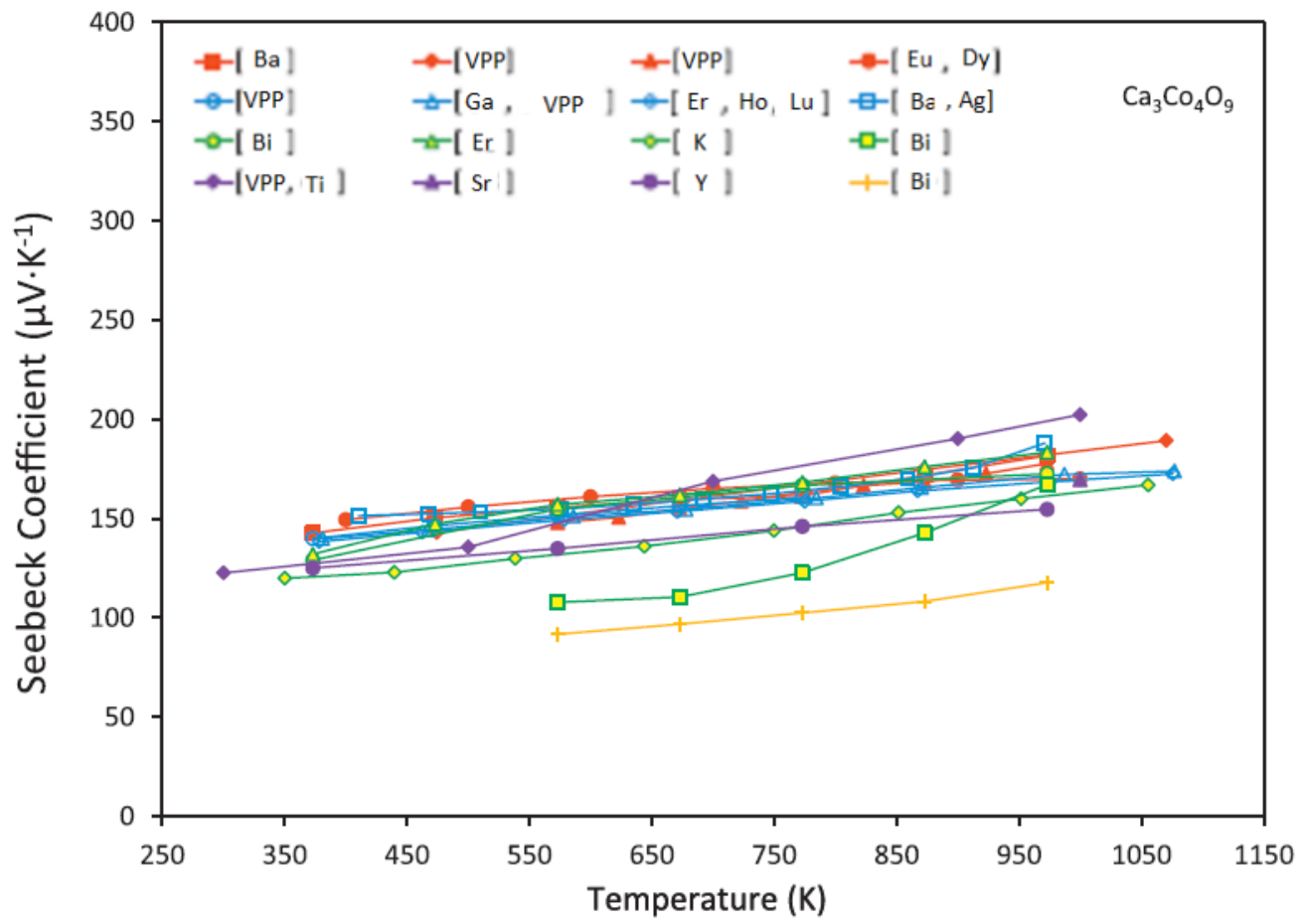

Figure 9 Absolute Seebeck coefficient for $\mathrm{Ca}_{3} \mathrm{C}_{4} \mathrm{O}_{9}$ with various dopants. [39]

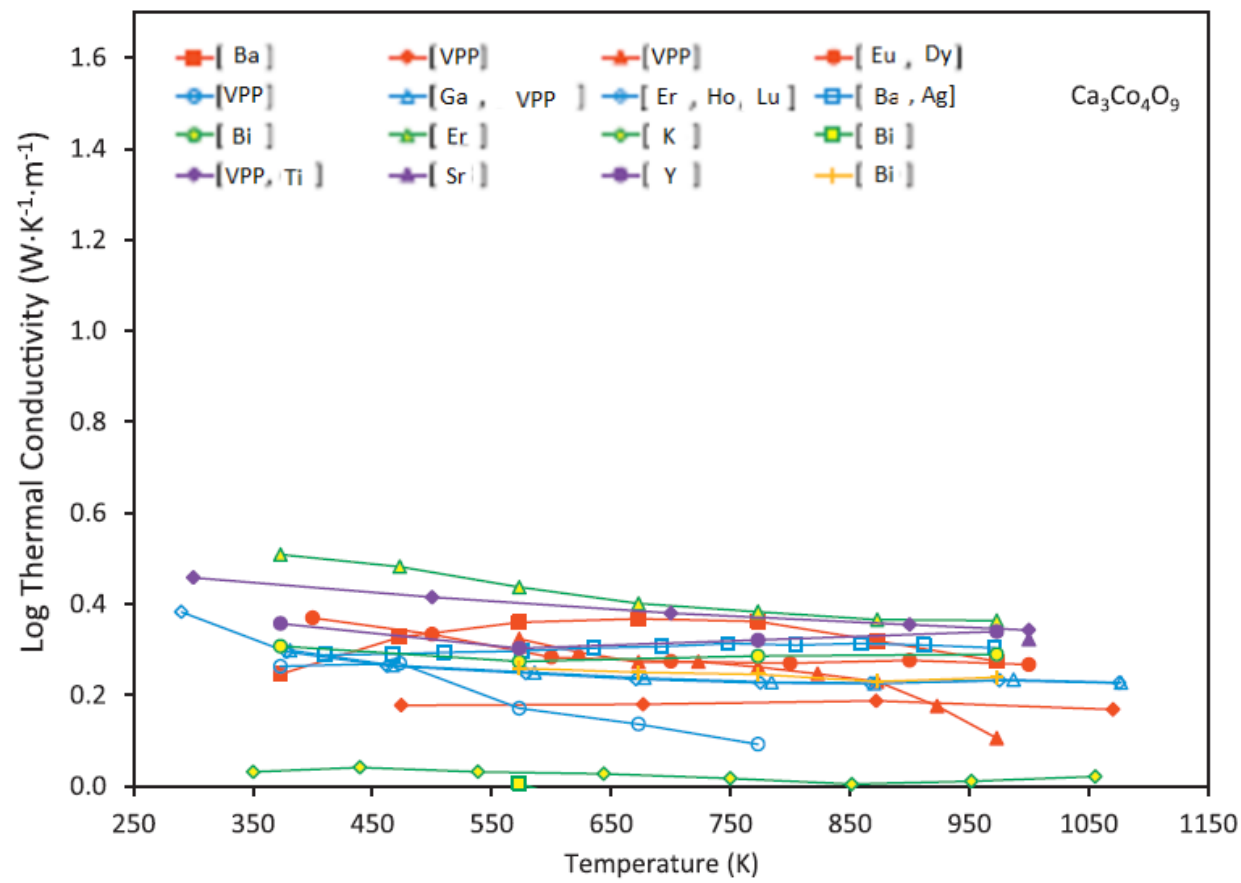

Figure 10 Log thermal conductivities of $\mathrm{Ca}_{3} \mathrm{Co}_{4} \mathrm{O}_{9}$ with various dopants. [39] 


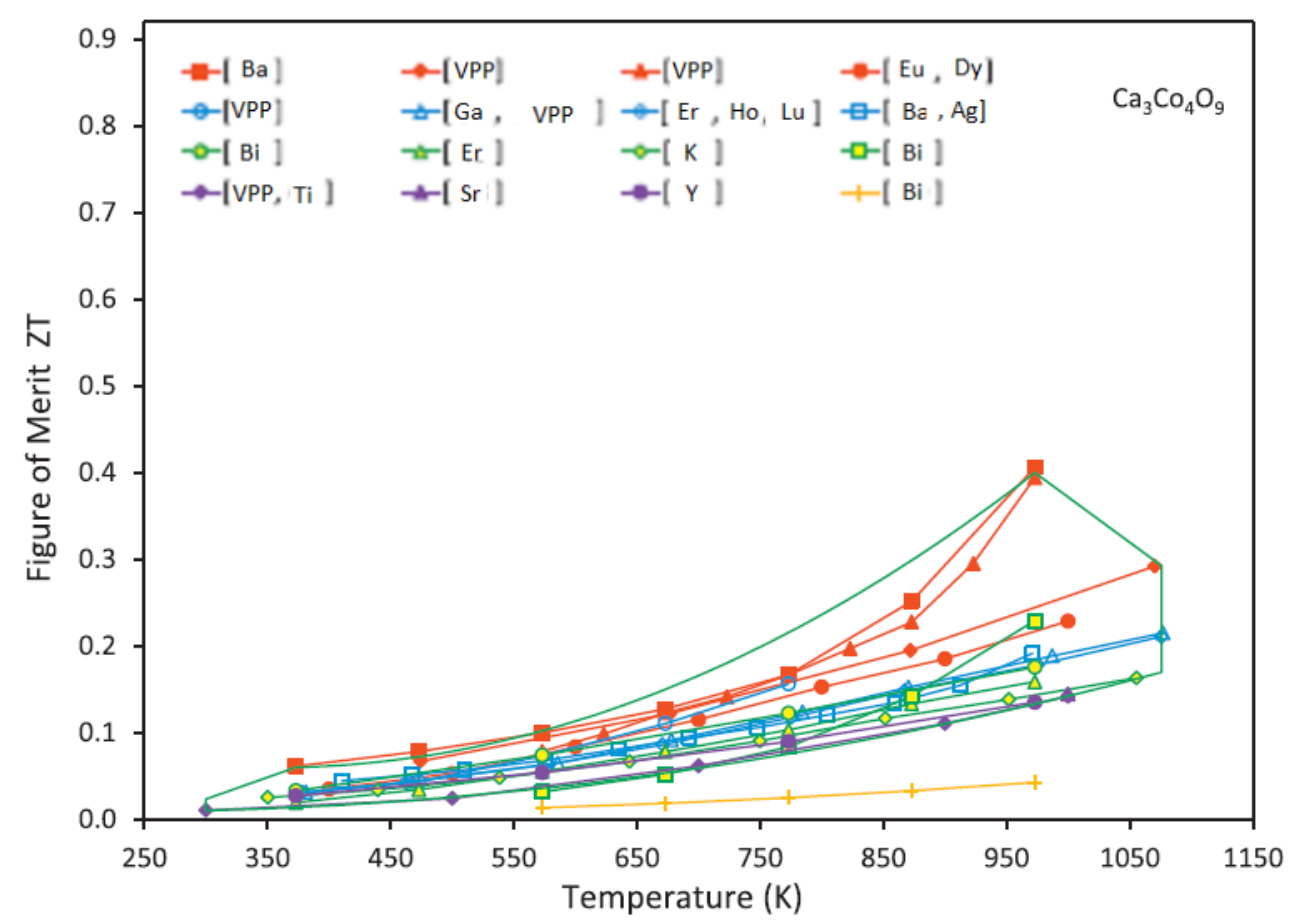

Figure 11 Figure of merit for $\mathrm{Ca}_{3} \mathrm{Co}_{4} \mathrm{O}_{9}$ with various dopants. ${ }^{[39]}$

\subsubsection{Crystal Structure and anisotropic nature of $\mathrm{Ca}_{3} \mathrm{C}_{4} \mathrm{O}_{9}$}

Calcium Cobaltite, $\mathrm{Ca}_{3} \mathrm{C}_{4} \mathrm{O}_{9}$, is an intrinsic semiconductor that when doped with impurities takes on extrinsic properties, which in turn will alter its electrical and thermal properties. It is a p-type Co-based misfit layered oxide consisting of two sub-systems. $[30,34,34,33]$ The misfit layers are stacked in the c-direction having the same a, c, $\beta$ parameters but different b parameters making it misfit along the b-axis. These alternating commensurate layers can be seen in the schematic shown in figure 12. The first layer is the outer $\mathrm{CoO}_{2}$ layers which form a triangular lattice and act as conductive layers providing the electronic transport pathway. ${ }^{[30,34,34]}$ The second layer, sandwiched between the conducting layers, is the distorted rock-salt type $\mathrm{Ca}_{2} \mathrm{CoO}_{3}$ which forms a 
rectangular lattice and is the insulating layer, responsible for phonon-scattering, lowering its thermal properties. ${ }^{[30,34,34]}$ In this regard it has a lattice constant and lattice geometry misfit.

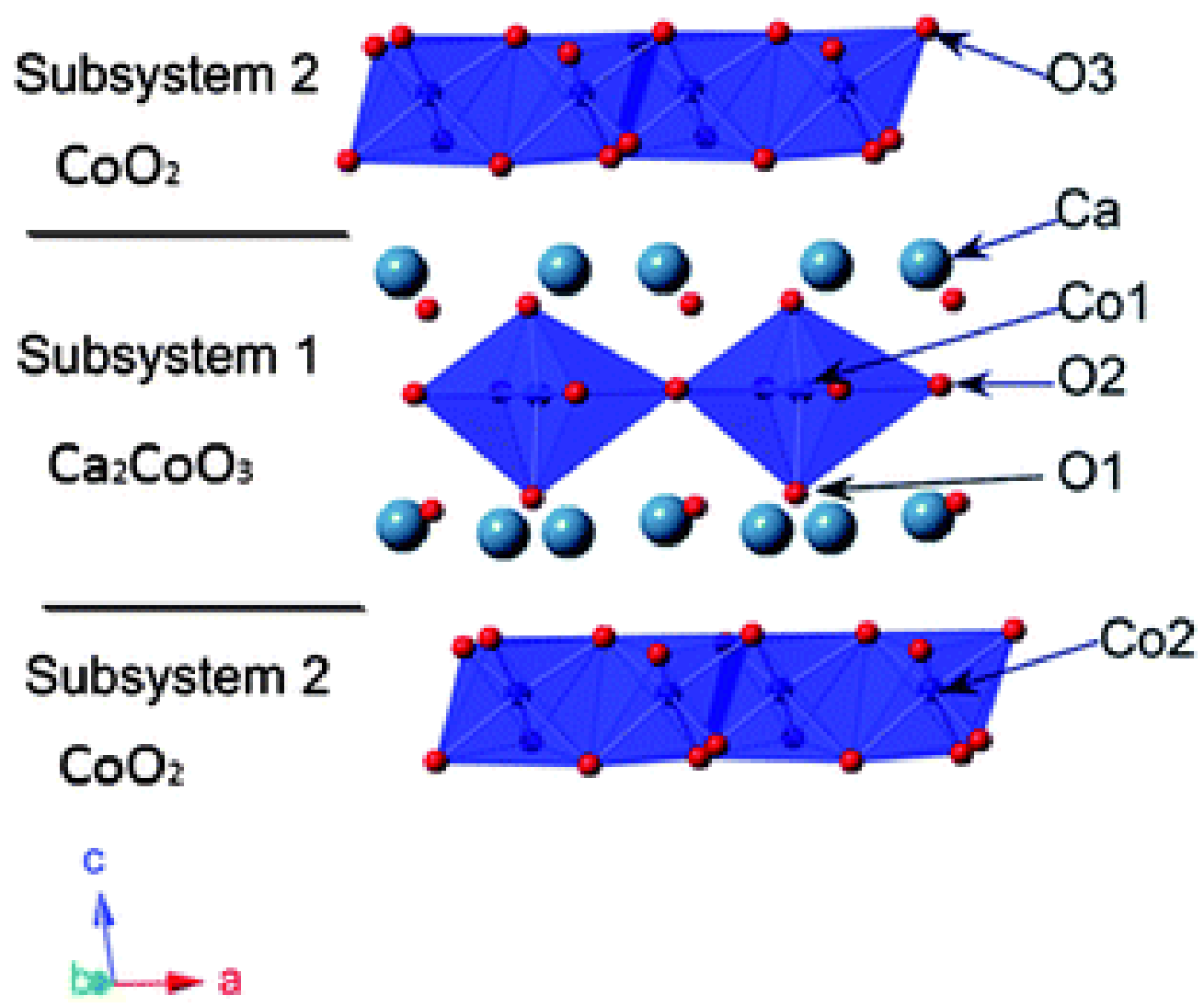

Figure 12 Schematic of alternating subsystems of $\mathrm{Ca}_{3} \mathrm{Co}_{4} \mathrm{O}_{9}$ crystal structure [40]

As mentioned Calcium Cobaltite's single crystal has a relatively high figure of merit due to its significant grain alignment in the c-direction. This is the direction of the stacking of the alternating sub-systems. The polycrystalline form however, suffers from misalignment of these crystals thus having a high electrical resistivity. The challenge for this material is to improve the thermoelectric performance, or $\mathrm{ZT}$, of the polycrystalline ceramic. This can be done by improving its electrical properties through grain alignment 
which in turn will improve its overall power factor. Furthermore, by reducing the thermal conductivity of the material through phonon scatter the figure of merit, $\mathrm{ZT}$, can be increased as well.

Grain alignment is measured through what is called the Lotgering factor and given a value between 0 and 1. ${ }^{[41]}$ Material with complete disorder is represented by a Lotgering factor of 0 and materials with complete order have a factor of 1 . The Calcium Cobaltite's baseline has a Lotgering factor of about 0.74 . ${ }^{[27,41]}$ Although grain growth can be beneficial it can have a downside as well. If the grain growth is too rapid and significant the grains can have a difficult time aligning themselves during the sintering process and can cause the lattice to expand, reducing the density and negatively affecting the material's electrical resistivity. This growth also causes misalignment in the grains and does not allow for smooth transport of the electron-hole pair.

The $\mathrm{Ca}_{3} \mathrm{Co}_{4} \mathrm{O}_{9}$ material is anisotropic, having different values for its lattice depending on which direction it is measured in. This has to do with its unique stacking structure. Not only do the stacks alternate in the c-direction, but this causes each individual insulating layer to have different dimensions from its neighboring conductive layer, thus giving it two different $b$-direction values, $b_{1}$ and $b_{2}$. This misalignment at the boundaries can be a platform for phonon scattering events, leading to a low thermal conductivity. This also tends to create misalignment throughout the material. These commensurate b-direction values can be seen in figure 13, labeled as b'coo2 and b'Rs. In order to properly bond the alternating layers one must compress thus creating undulations within this layer while the other expands. 


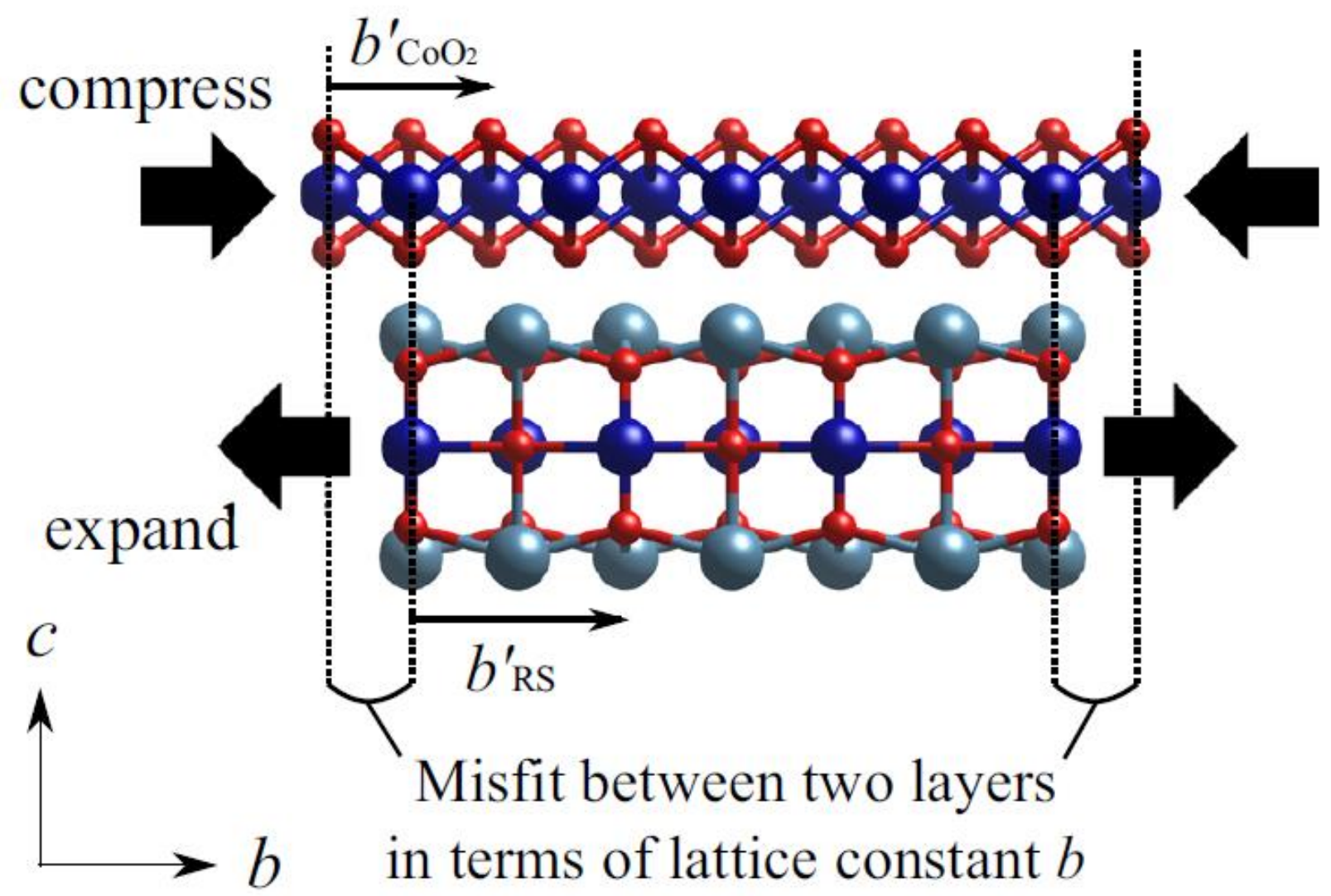

Figure 13 Schematic of lattice misfit. ${ }^{[34]}$

1.5.3 Current Status of $\mathrm{Ca}_{3} \mathrm{C}_{4} \mathrm{O}_{9}$ Thermoelectric Performance Reported in Literatures A large issue pertaining to this research is the expense of the processing of some of the materials. Solid-State-Reaction, SSR, is most widely used among researchers but does not particularly allow the proper control of the stoichiometric ratios or the same uniformity that the sol-gel route has to offer. SSR is most commonly used due to its ease in the lab and requires the ball milling of the precursors which are then immediately calcined after. ${ }^{[42,43]}$ The sol-gel route on the other hand requires stirring the precursors suspended in a mixture which aids in decomposition and polymerization to create a new compounds. ${ }^{[38]}$ The inorganic material is then burned off before it enters the calcination 
stage. This extra step allows the materials to rearrange their structures and create bonds before they reach their crystal structure during calcination. The sol-gel process for precursors allows for the exact chemistry which can be controlled from the beginning of the process to the pelletization of the powders. This ensures that end results reflect the original intentions of the chemistry. From here it is much easier to know how to change the parameters and which parameters to change. SSR also requires a higher temperature, for thermal polymerization, or multiple thermal treatments, in order to react together, constituting a higher calcination temperature ${ }^{[42]}$. A higher temperature can stimulate too much grain growth too early in the process creating a bulk material with a lower density and a higher electrical resistivity. SSR is also often accompanied by lattice deformation energy and strain. ${ }^{[44]}$ This process only exacerbates this material's preexisting lattice deformation which can be responsible for impeding its electrical properties as well.

Another variance in processing are the pressing parameters. Two main methods of pressing are through hot and cold pressing. A lot of the times the dopant involved will dictate which method is used. Certain materials react more favorably when exposed to heat during the pressing stage and can assist in the alignment of grains whether through their reaction and action to the heat or from the densification of a liquid phase achieved. More often than not the pressure or temperature involved in this stage predominantly effects the densification and texture of the pellet.

Spark plasma sintering, SPS, has been a commonly used practice for thermoelectric ceramic material. SPS uses pulses of DC current that pass through a graphite press. ${ }^{[45,46]}$ The purpose of the process is to increase density within the pellet 
during sintering. ${ }^{[45,46]}$ This can be beneficial for electrical purposes but can pose an issue when testing its thermal properties considering thermal conductivity is directly related to the material's density. The other benefit SPS has is that it only takes minutes to sinter since there is internal heat created through the current. This allows the temperature to ramp up quicker than a conventional furnace would for alternative methods. This high heating rate of SPS, allowing for less holding time, also reduces the possibility of unwanted sintering reactions. Aside from the shorter sintering time, SPS creates a bulk sample with a relatively higher density. ${ }^{[47]}$ Conventional sintering although more time consuming than SPS has less opportunity for cracking to occur in the sample due to the slower ramping temperature. Conventional sintering is the cheaper alternative of the two methods and is also highly reproducible. This is the chosen method for this study covered in this dissertation.

Previously alloy semiconductors, lead and antimony containing compounds such as $\mathrm{PbTe}, \mathrm{Sb}_{2} \mathrm{Te}_{3}$, and $\mathrm{Sb}_{2} \mathrm{Se}_{3}$ have been widely used as thermoelectric material. The benefits of utilizing these materials is that most are very robust and efficient at high temperatures. However, due to their instability and issues with oxidizing, some of the higher temperature operating materials need to be used in a vacuum. Silicon germanium for instance has a figure of merit of around 1 at temperatures around $1000 \mathrm{~K}$ and BiCuSeO figure of merit has been improved upon from 0.5 to 1.4 from 2010 to 2014 both needing a vacuum to achieve these values for a long timeline. ${ }^{24,38,48]}$ Both can be located in the graph in figure 14. Lead, $\mathrm{Pb}$ and antimony, $\mathrm{Sb}$, containing material yield very promising ZTs at mid to high temperatures but are toxic and not desirable to handle in the lab. The idea is to create a material that not only provides efficiency and a high electrical 
power, but also one that is stable and robust at high temperatures along with being structurally sound.

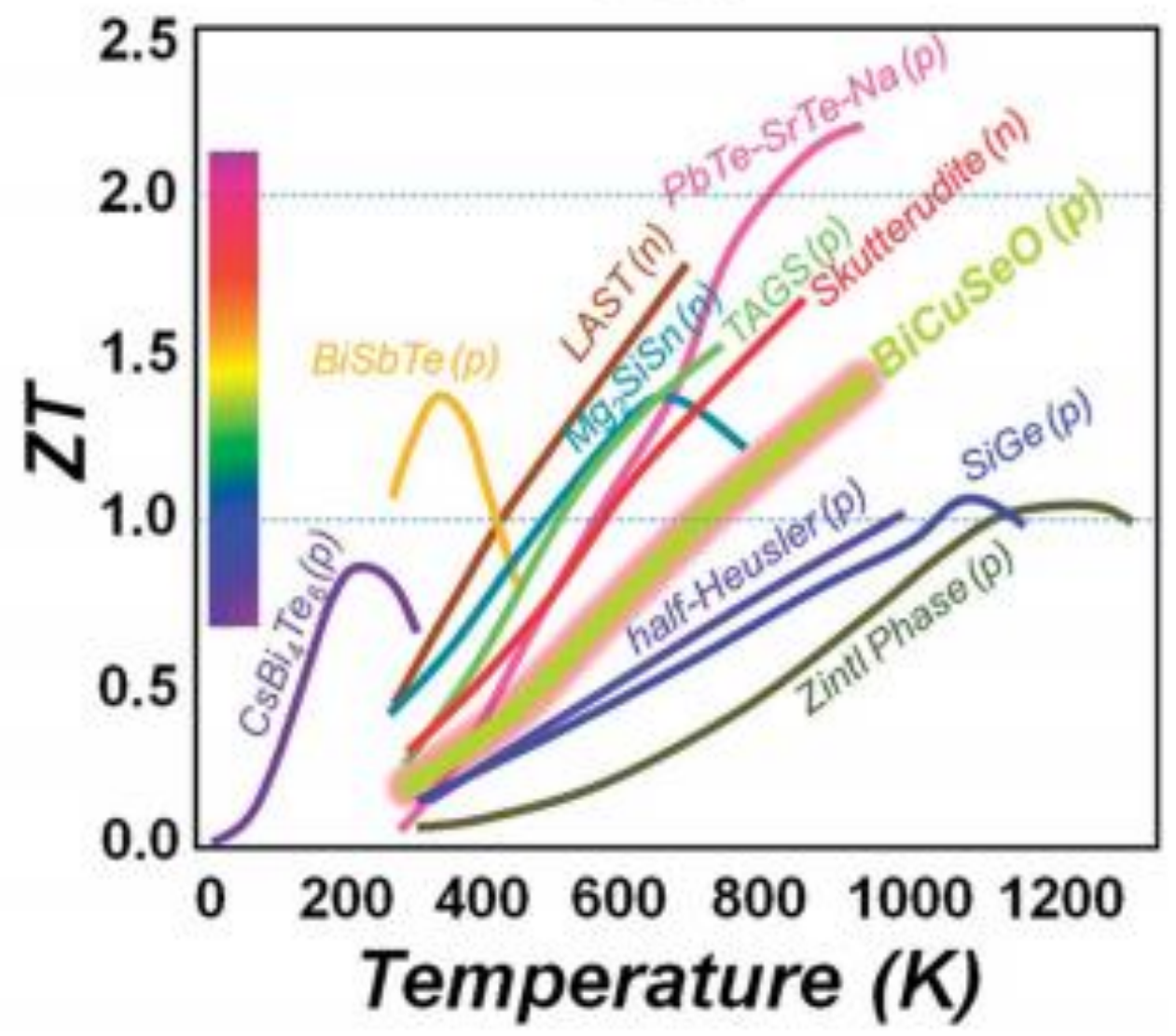

Figure 14 Dimensionless figure of merit vs. temperature of typical current thermoelectric materials. ${ }^{[48]}$

For the purpose of this dissertation it is important to approach the efficiency of silicon germanium in order to be competitive on a large scale. Some other materials may yield a figure of merit well over 1 but once again they either operate at low temperatures or the material can be toxic or pricey due to its rare status. Since these achievements, oxides have made substantial leaps in this field to finally be competitive with their costly 
and toxic counterparts. Rare-earth elements such as $\mathrm{Yb}, \mathrm{Lu}, \mathrm{Pr}, \mathrm{Nd}, \mathrm{Gd}$, and Eu have recently been used to dope oxides to achieve great success. ${ }^{[49,50,51}$ Oxides have improved vastly over the years but still suffer from inferior ZTs comparted with alloys alike. The highest ZT to date for oxides was reported by Shrikant Saini et al through Terbium lon doping of $\mathrm{Ca}_{3} \mathrm{Co}_{4} \mathrm{O}_{9}$ with a record $\mathrm{ZT}$ of 0.74 at $800 \mathrm{~K}$. ${ }^{[52]}$ This is the highest value reported for any polycrystalline sample. ${ }^{[52]}$ this work proves that oxides still have a place in this particular research and have plenty of room to grow through processing and doping. 


\subsection{References}

1 Yi-Cheng Hou, Mei-Jiau Huang, Pi-Yueh Chuang, Hsiu-Cheng Chang, Chun-Hua Chen. Numerical and model predictions of the thermal conductivity of bismuth telluride nanoprism-assembled films. International Journal of heat and Mass Transfer 87. 536543. April 13, 2015

2 Kasper A. Borup, Johannes de Boor, Heng Wang, Fivos Drymiotis, Franck Gascoin, Xun Shi, Lidong Chen, Mikhail I. Fedorov, Eckhard Muller, Bo B. Iversen and G. Jeffrey Snyder. Measuring thermoelectric transport properties of materials. Energy and Environmental Science. 8 pp.423-434. September 23, 2014.

3 Materials Science. Division of Engineering \& Applied Science. California Institute of Technology. 2017

4 Michitaka Ohtaki. Recent aspects of oxide thermoelectric materials for power generation from mid-to-high temperature heat source. Journal of the Ceramic Society of Japan 119 [11] 2011.

5 Ashok Kumar Yadav, Shatrughan Singh, Gaurav Gupta. Solar Air-Conditioning: Design for a Compressor-Less System using Peltier Effect. International Journal of Advance Research and Innovation. Vol. 2, Iss. 2, pp. 429-432, 2014.

6 Daniel Kraemer, Bed Poudel, Hsien-Ping Feng, J. Christopher Caylor, Bo Yu, Xiao Yan, Yi ma, Xiaowei Wang, Dezhi Wang, Andrew Muto, Kenneth McEnaney, Matteo Chiesa, Zhifeng Ren and Gang Chen. High-performance flat-panel solar thermoelectric generators with high thermal concentration. Mature Materials 10, 532-538. May 1, 2011

7 Mahmoud Dhimish, Violeta Holmes, Mark Dales. Parallel fault detection algorithm for grid-connected photovoltaic plants. Renewable Energy. Vol. 113, pp. 94-111, December 2017.

8 Independent Statistics \& Analysis. U.S. Energy Information Administration. (2016).

9 Xiao zhang, Li-Dong Zhao. Thermoelectric materials: Energy conversion between heat and electricity. Journal of Materiomics. Vol.1, pp.92-105, 2015.

10 T. Sun, H. H. Hng, Q. Y. Yan, and J. Ma. Enhanced high temperature thermoelectric properties of $\mathrm{Bi}$-doped $\mathrm{c}$-axis oriented $\mathrm{Ca}_{3} \mathrm{C}_{4} \mathrm{O}_{9}$ thin films by pulsed laser deposition. Journal of Applied Physic 108. AIP Publishing. 2010.

${ }^{11}$ T. Sun, H. H. Hng, Q. Y. Yan, and J. Ma. Enhanced high temperature thermoelectric properties of $\mathrm{Bi}$-doped c-axis oriented $\mathrm{Ca}_{3} \mathrm{C}_{4} \mathrm{O}_{9}$ thin films by pulsed laser deposition. Journal of Applied Physics. Vol.108, 2010.

12 Past, Present, and Future of Solar Energy. Cleaner Green Green solutions for today. Dan Clem. April 2012.

${ }^{13}$ Nong Ngo Van and Nini Pyrds. Nanostructured oxide materials and modules for hightemperature power generation from waste heat. Advances in Natural Sciences: Nanoscience and Nanotechnology. 4 March 25, 2013. 
14 Mohamed Hamid Elsheikh, Dhafer Abdulameer Shnawah, Mohd Faizul Mohd Sabri, Suhana Binti Mohd Said, Masjuki Haji Hassan, Mohamed Bashir Ali Bashir, Mahazanti Mohamad. A review on thermoelectric renewable energy: Principle parameters that affect their performance. Renewable and Sustainable Energy Reviews. Vol. 30, pp.337-355. 2014.

15 Intrinsic and Extrinsic Semiconductors. DolTPoMS. University of Cambridge. 20042015

16 Donor and Acceptor Impurities in Semiconductor. Electrical4u. 2011-2017.

17 Ananya Ganguly. Fundamentals of Physical Chemistry for Competitive Examinations. Dorling Kindesley Pearson Education, pp. 40-41, 2011

18 Dragica Vasileska, Stephen M. Goodnick, Gerhard Klimeck. Computational Electronics: Semiclassical and Quantum Device Modeling and Simulation. CRC Press, pp. 34-35, 2016.

${ }^{19}$ Chong Xiao, Jie Xu, Boxiao Cao, Kun Li, Mingguang Kong, and Yi Xie. Solid-solutioned homojunction nanoplates with disordered lattice: A promising approach toward "Phonon Glass Electron Crystal" thermoelectric materials. Journal of the American Chemical Society 134, pp.7971-7977. April 23, 2012.

20 Michael P. Rowe, Li Qin Zhou, Debasish Banerjee, and Minjuan Zhang. Improvement of the thermoelectric figure-of-merit of a doped telluride nanocomposite by combining phonon scattering with grain boundary-modifying Zn-containing nanostructures. Journal of Electronic Materials, Vol.44, No.1, 2015.

${ }^{21}$ Tao Wu, Trevor A. Tyson, Haiyan Chen, Jianming Bai, Hsin Wang and Cherno Jaye. A structural phase transition in $\mathrm{Ca}_{3} \mathrm{Co}_{4} \mathrm{O}_{9}$ Associated with enhanced high temperature thermoelectric properties. Cornell University Library. April 15, 2012.

22 T. Takeuchi, T. Kondo, K. Soda, U. Mizutani, R. Funahashi, M. Shikano, S. Tsuda, T. Yokoya, S. Shin, T. Muro. Electronic structure and large thermoelectric power in $\mathrm{Ca}_{3} \mathrm{Co}_{4} \mathrm{O}_{9}$. Journal of Electron Spectroscopy and Related Phenomena 137-140 2004 595-599. March 18, 2004.

23 Hee Seok Kim, Weishu Liu, G Hang Chen, Ching-Wu Chu, and Zhifeng Ren. Relationship between thermoelectric figure of merit and energy conversion efficiency. Proceedings of the National Academy of Sciences of the United States of America. June 22, 2015.

24 Materials Science and Engineering. McCormick School of Engineering. Northwestern University 2017.

25 Haoshan Hao, Qinglin He, ChangQing Chen, Hongqei Sun and Xing Hu. Textured structure and anisotropic thermoelectric properties of $\mathrm{Ca}_{2.7} \mathrm{Bi}_{0.3} \mathrm{C}_{0} \mathrm{O}_{9}$ oxide prepared by conventional solid-state reaction. International Journal of Modern Physics B Vol. 23, No.1. World Scientific Publishing Company. 2009.

${ }^{26}$ Guodong Tang, Wenchao Yang, Jianfeng Wen, Zhuangchun Wu, Cang Fan, Zhihe Wang. Ultralow thermal conductivity and thermoelectric properties of carbon 
nanotubes doped $\mathrm{Ca}_{3} \mathrm{C}_{4} \mathrm{O}_{9+\delta}$. Ceramics International 41, pp.961-965. September 15, 2014.

27 S. Demirel, E. Altin, E. Oz, S. Altin, A. Bayri. An enhancement ZT and spin state transition of $\mathrm{Ca}_{3} \mathrm{CO}_{4} \mathrm{O}_{9}$ with $\mathrm{Pb}$ doping. Journal of Alloys and Compounds 627 pp.430437. December 27, 2014.

${ }^{28}$ A. Sotelo, F. M. Costa, N. M. Ferreira, A. Kovalevsky, M. C. Ferro, V. S. Amaral, J. S. Amaral, Sh. Rasekh, M. A. Torres, M. A. Madre, J. C. Diez. Tailoring $\mathrm{Ca}_{3} \mathrm{Co}_{4} \mathrm{O}_{9}$ microstructure and performances using a transient liquid phase sintering additive. Journal of the European Ceramic Society 36, pp.1025-1032. November 30, 2015.

${ }^{29}$ Lijun Wu, inping Meng, Christian Jooss, Jin-Cheng Zheng, H. Inada, Dong Su, Quang $\mathrm{Li}$, and Yimei Zhu. Origin of phonon glass-electron crystal behavior in thermoelectric layered cobaltate. Advanced Functional Materials Journal 23, 5728-5736. 2013.

${ }^{30}$ Guangkun Ren, Jinle Lan, Chengcheng Zeng, Yaochun Liu, Bin Zhan, Sajid Butt, YuanHua Lin, and Ce-Wen Nan. High performance oxides-based thermoelectric materials. Journal of the Minerals, Metals, \& Materials Society, Vol.67, No.1, 2015.

${ }^{31}$ NanoEngineering Group. Massachusetts Institute of Technology

32 Kasper A. Borup, Johannes de Boor, Heng Wang, Fivos Drymiotis, Franck Gascoin, Xun Shi, Lidong Chen, Mikhail I. Fedorov, Eckhard Muller, Bo B. Iversen and G. Jeffrey Snyder. Measuring thermoelectric transport properties of materials. Energy and Environmental Science. 8 pp.423-434. September 23, 2014.

${ }^{33}$ Sajid Butt, Yaoyu Ren, Muhammad Umer Farooq, Bin Zhan, Rizwan Ur Rahman Sagar, Yuanhua Lin, Ce-Wen Nan. Enhanced thermoelectric performance of heavy-metals (M: $\mathrm{Ba}, \mathrm{Pd})$ doped misfit-layered ceramics: $\left(\mathrm{Ca}_{2}-\mathrm{x} \mathrm{M}_{\times} \mathrm{CoO}_{3}\right)_{0.62}\left(\mathrm{CoO}_{2}\right)$. Energy Conversion and Management. Vol. 83, pp. 35-41, 2014.

${ }^{34}$ Susumu Fujii and Masato Yoshiya. Manipulating thermal conductivity by interfacial modification of misfit-layered cobaltites $\mathrm{Ca}_{3} \mathrm{C}_{4} \mathrm{O}_{9}$.

35 Masashi Mikami, Kanji Chong, Yuzuru Miyazaki, Tsuyoshi Kajitani, Takahiro Inoue, Satoshi Sodeoka, and Ryoji Funahashi. Bi-substitution effects on crystal structure and thermoelectric properties of $\mathrm{Ca}_{3} \mathrm{Co}_{4} \mathrm{O}_{9}$ single crystals. Japanese Journal of Applied Physics. Vol.45, No.5A, pp. 4131-4136, 2006.

${ }^{36}$ Chang-Hyun Lim, Won-Seon Seo, Soonil Lee, Young Soo Lim, Jong-Young Kim, Hyung-Ho Park, Soon-Mok Choi, Kyu Hyoung Lee, Kyeongsoon Park. Anisotropy of the Thermoelectric Figure of Merit (ZT) in Textured $\mathrm{Ca}_{3} \mathrm{CO}_{4} \mathrm{O}_{9}$ Ceramics Prepared by using a Spark Plasma Sintering Process. Journal of the Korean Physical Society, Vol. 66, pp. 794-799, March 2015.

${ }^{37}$ T. Takeuchi, T. Kondo, K. Soda, U. Mizutani, R. Funahashi, M. Shikano, S. Tsuda, T. Yokoya, S. Shin, T. Muro. Electronic structure and large thermoelectric power in $\mathrm{Ca}_{3} \mathrm{Co}_{4} \mathrm{O}_{9}$. Journal of Electron Spectroscopy and Related Phenomena 137-140 pp.595-599. March 18, 2004.

${ }^{38}$ Cullen Boyle, Paulo Cavillo, Yun Chen, Ever J. Barbero, Dustin McIntyre, Xueyan Song. Grain boundary segregation and thermoelectric performance enhancement of bismuth 
doped calcium cobaltite. Journal of the European Ceramic Society. Vol. 36, pp. 601607, 2016.

39 Jeffrey W. Fergus. Oxide materials for high temperature thermoelectric energy conversion. Journal of the European Ceramic Society. 32, pp. 525-540, 2012.

40 Tao Wu, Trevor A. Tyson, Jianming Bai, Kaumudi Pandya, Cherno Jaye, and Daniel Fischer. On the origin of enhanced thermoelectricity in $\mathrm{Fe}$ doped $\mathrm{Ca}_{3} \mathrm{CO}_{4} \mathrm{O}_{9}$. Journal of Materials Chemistry C. Vol. 1, pp. 4114-41212, 2013.

41 Ruomin Tian, Tianshu Zhang, Dewei Chu, Richard Donelson, Li Tao, Sean Li. Enhancement of high temperature thermoelectric performance in $\mathrm{Bi}, \mathrm{Fe}$ co-doped layered oxide-based material $\mathrm{Ca}_{3} \mathrm{Co}_{4} \mathrm{O}_{9}$. Journal of Alloys and Compounds. 615:311315. July 4, 2014.

42 Ankam Bhaskar, Z.-R. Lin, Chia-Jyi Liu. Thermoelectric properties of Ca2.95Bio.05Co4${ }_{x} \mathrm{Fe}_{x} \mathrm{O}_{9+\delta}(0<\mathrm{x}<0.15)$. Energy Conversion and Management. Vol. 76, pp. 63-67, 2013.

${ }^{43}$ Rezaul Kabir, Ruoming Tian, Tianshu Zhang, Richard Donelson, Thiam Teck Tan, Sean $\mathrm{Li}$. Role of $\mathrm{Bi}$ doping in thermoelectric properties of $\mathrm{CaMnO}_{3}$. Journal of Alloys and Compounds. Vol. 628, pp. 347-351, 2015.

44 Tadeusz Luty and Craig J. Eckhardt. General Theoretical Concepts for Solid State Reactions: Quantitative Formulation of the Reaction Cavity, Steric Compression, and Reaction-Induced Stress Using an Elastic Multipole Representation of Chemical Pressure. Journal of American Chemical Society. Vol. 117, pp. 2441-2452, 1995.

${ }^{45}$ H. Q. Liu, X. B. Zhao, T. J. Zhu, Y. Song, F. P. Wang. Thermoelectric properties of Gd, Y co-doped $\mathrm{Ca}_{3} \mathrm{Co}_{4} \mathrm{O}_{9+\delta}$. Current Applied Physics. Vol. 9, pp. 409-413, 2009.

46 NingYu Wu, Tim C. Holgate, Ngo Van Nong, Nini Pryds, Søren Linderoth. High temperature thermoelectric properties of $\mathrm{Ca}_{3} \mathrm{C}_{4} \mathrm{O}_{9+\delta}$ by auto-combustion synthesis and spark plasma sintering. Journal of the European Ceramic Society. Vol. 34, pp. 925-931, 2014.

47 Ana Júlia Tertuliano, Guido Boidi, Daniel Rodrigues, Izabel Fernanda Machado. Comparison between a conventional sintering process and by SPS (Spark Plasma Sintering) consolidation of WC-6Co (\%wt). Brazilian Congress of Engineering and Material Science. Pp. 2042-2047, 2014.

48 Li-Dong Zhao, Jiaqing He, David Berardan, Yuanhua Lin, Jing-Feng Li, Ce-Wen Nan, and Nita Dragoe. BiCuSeO oxyselenides: new promising thermoelectric materials. Energy \& Environmental Science. Vol. 7, pp. 2900-2924, 2014.

49 C.S. Huang, F.P.Zhang, X. Zhang, Q. M. Lu, J. X.Zhang, Z. Y. Liu. Enhanced thermoelectric figure of merit through electrical and thermal transport modulation by dual-doping and texture modulating for $\mathrm{Ca}_{3} \mathrm{Co}_{4} \mathrm{O}_{9+\delta}$ oxide materials. Journal of Alloys and Compounds. 687, pp. 87-94, 2016.

50 Yang Wang, Luxiang $\mathrm{Xu}$, Yu Sui, Xianjie Wang, Jinguang Cheng, and Wenhui Su. Enhanced electron correlation in rare-earth doped $\mathrm{Ca}_{3} \mathrm{Co}_{4} \mathrm{O}_{9}$. Applied Physics Letters. 97, 2010. 
51 Nong Ngo Van and Nini Pryds. Nanostructured oxide materials and modules for hightemperature power generation from waste heat. Advances in Natural Sciences: Nanoscience and Nanotechnology. Vol. 4, 2013.

52 Shrikant Saini, Haritha Sree Yaddanapudi, Kun Tian, Yinong Yin, David Magginetti \& Ashutosh Tiwari. Terbium Ion Doping in $\mathrm{Ca}_{3} \mathrm{C}_{4} \mathrm{O}_{9}$ : A Step towards High-Performance Thermoelectric Materials. Nature. 2016. 


\section{Chapter 2:}

\section{Research Objectives and Methodology}

\subsection{Objectives}

Over $80 \%$ of the United States' electrical energy comes from fossil fuels which is the major source of $\mathrm{CO}_{2}$ emissions leading to global warming. ${ }^{[1]}$ Furthermore, these natural resources are limited and threaten to eventually be exhausted. Scientists have been working on an answer for years, utilizing many different methods and thoughts for renewable energy and waste energy recovery. For as far back as one can search the holy grail for scientists and inventors has been the fabled "perpetual motion machine". ${ }^{[2]}$ It is a device that will run forever without the loss of energy. In theory a perpetual motion machine works with $100 \%$ efficiency and no waste heat at all. ${ }^{[2,3]}$ This is impossible from a physics standpoint due to the second law of thermodynamics. The second law states that waste heat must always be produced thus increasing entropy in the Universe. ${ }^{[2]}$

One approach widely used today is the harvesting of this unused energy. One of the earliest and probably most recognizable forms of harvesting energy would be that of solar panels which use the Sun's light as its source of energy and were first used in space in 1958 and later introduced to the commercial market in the 70 s. ${ }^{[4]}$ The use of solar panels is an example of the photovoltaic process. Since then, many attempts have been made to utilize this same concept of harnessing waste heat and transforming it into usable energy. A second approach is through thermoelectric generation. As stated in the previous chapter TEG are used to harness and repurpose energy through waste heat. 
Oxides as a thermoelectric material provide a much greater alternative than the long used alloy semiconductors. As of now their efficiencies are still inferior in comparison but they hold great promise due to their low toxicity, low cost, ease of use, thermal and chemical stability, and their ability to operate in air. Oxides are quickly catching up in their thermoelectric performance and more and more research has been dedicated to altering their micro- and nanostructures, in order to boost their efficiency. Their robust nature and ability to operate at high temperatures allow them to have a bright future in this field. Calcium cobaltite is in the forefront of that research right now with plenty of room to improve upon.

The main sources of harvestable energy have been through mechanical vibrations and waste heat. Both byproducts that go unused and a lot of times unnoticed. Harvesting energy through waste heat is what this paper will mainly focus on. More specifically, the method discussed in this dissertation will be by use of a TEG pertaining to waste heat. It will cover what a TEG is, what it does, and how it works. It will also describe the components that comprise the device and how they work as well.

The objective of this research is to improve the thermoelectric performance of Calcium Cobaltite ceramics by modifying its chemistry. This was done through conventional sol-gel processing for precursors and conventional pelletization and sintering. It will investigate different doping elements along with different methods of introducing these dopants into the $\mathrm{Ca}_{3} \mathrm{C}_{4} \mathrm{O}_{9}$ ceramic. The effects of the chemistry modification on its thermoelectric performance and nanostructure will be extensively investigated to understand the correlation between the two induced by the chemistry modification. 


\subsection{Methodology}

Three different dopants in the $\mathrm{Ca}_{3} \mathrm{Co}_{4} \mathrm{O}_{9}$ are investigated in this dissertation. Chapter three focuses on the first which is the addition of the Chalcogen bismuth telluride, $\underline{\mathrm{Bi}_{2} \mathrm{Te}_{3}}$ in the $\mathrm{Ca}_{3} \mathrm{Co}_{4} \mathrm{O}_{9}$. This was achieved by mixing stoichiometric measurements of $\mathrm{Ca}_{3} \mathrm{C}_{4} \mathrm{O}_{9}$ then adding the $\mathrm{Bi}_{2} \mathrm{Te}_{3}$ in a second stage ball mill. The motivation of this set is to investigate whether the addition of bismuth telluride forms an alloy or a composite with $\mathrm{Ca}_{3} \mathrm{C}_{4} \mathrm{O}_{9}$. Bismuth telluride was doped into the $\mathrm{Ca}_{3} \mathrm{C}_{4} \mathrm{O}_{9}$ in order to analyze the possibility of grain boundary segregation. The formation of a secondary phase using bismuth telluride as a dopant is also very possible and was analyzed as well.

Chapter four introduces the second dopant which covers the cationic substitution of bismuth into the $\mathrm{Ca}_{3} \mathrm{C}_{4} \mathrm{O}_{9}$. Bismuth has a high atomic mass which should help to increase the density of the material to enhance its electrical properties and create scattering events in order to reduce its thermal conductivity. Bismuth is also known to be the most naturally occurring diamagnetic element in nature allowing it to have the lowest thermal conductivity of all metals.

Chapters five and six discuss the use of non-stoichiometric addition of dopants into the $\mathrm{Ca}_{3} \mathrm{Co}_{4} \mathrm{O}_{9}$ chemistry. The motivation was to investigate the thermoelectric properties of a chemistry where the dopant will most likely not replace calcium in the lattice and not deposit itself at the grain interior. With this approach the stoichiometry was purposely not preserved in order to analyze where such a dopant presents itself and how it affects the overall performance of the material. In chapter five, bismuth was once again used to dope the $\mathrm{Ca}_{3} \mathrm{C}_{4} \mathrm{O}_{9}$. Bismuth's ionic radius is close in size to that of 
calcium's but with no subtraction of the calcium the bismuth should have nowhere else to go but the grain boundary. The motivation was to explore whether the bismuth still finds its way into the lattice or is deposited elsewhere. Chapter six introduces the third and final dopant, discussing the doping of $\mathrm{Ca}_{3} \mathrm{C}_{4} \mathrm{O}_{9}$ with barium to further investigate this non-stoichiometric addition. Barium has a larger ionic radius than calcium and does not deposit in the lattice whether there are calcium deficiencies or not thus leaving it nowhere to go but the grain boundary. Barium is known to have a high electrical conductivity and is considered a heavy element which can cause phonon scattering to decrease the thermal conductivity positively affecting the overall performance.

Chapters seven, eight, and nine investigate the dual doping of both bismuth and barium into the $\mathrm{Ca}_{3} \mathrm{C}_{4} \mathrm{O}_{9}$ polycrystalline powders. Chapter seven discusses the non-stoichiometric addition of bismuth and the non-stoichiometric addition of barium while chapter eight and nine addresses the cationic substitution of bismuth and the nonstoichiometric addition of barium.

All chemistries in this dissertation began through a sol-gel stage of precursors with conventional methods of pelletization. Not only was this process affordable it was controllable and highly reproducible. Pellets were tested using a Linseis LSR-1100 to attain their electrical resistivities and Absolute Seebeck coefficients and a Linseis LFA1200 laser flash machine was used to acquire their thermal conductivities. Fractured pieces of the sample pellets were then further analyzed by their microstructures through a Hitachi Scanning Electron Microscope and PANalytical X-Ray Diffraction and their nanostructures analyzed by way of Transmission Electron Microscope. 


\subsection{References}

1 Independent Statistics \& Analysis. U.S. Energy Information Administration. (2016).

2 Kaku, Michio. Physics of the Impossible. 2008

3 The Laws of Thermodynamics.

4 The History of Solar. U.S. Department of Energy. Energy Efficiency and Renewable Energy.

5 Materials Science. Division of Engineering \& Applied Science. California Institute of Technology. 2017

6 The History of Solar. U.S. Department of Energy. Energy Efficiency and Renewable Energy.

7 Michitaka Ohtaki. Recent aspects of oxide thermoelectric materials for power generation from mid-to-high temperature heat source. Journal of the Ceramic Society of Japan 119 [11] 2011.

8 Yi-Cheng Hou, Mei-Jiau Huang, Pi-Yueh Chuang, Hsiu-Cheng Chang, Chun-Hua Chen. Numerical and model predictions of the thermal conductivity of bismuth telluride nanoprism-assembled films. International Journal of heat and Mass Transfer 87. 536543. April 13, 2015

9 Kasper A. Borup, Johannes de Boor, Heng Wang, Fivos Drymiotis, Franck Gascoin, Xun Shi, Lidong Chen, Mikhail I. Fedorov, Eckhard Muller, Bo B. Iversen and G. Jeffrey Snyder. Measuring thermoelectric transport properties of materials. Energy and Environmental Science. 8 pp.423-434. September 23, 2014.

${ }^{10}$ Nong Ngo Van and Nini Pyrds. Nanostructured oxide materials and modules for hightemperature power generation from waste heat. Advances in Natural Sciences: Nanoscience and Nanotechnology. 4 March 25, 2013.

${ }^{11}$ Daniel Kraemer, Bed Poudel, Hsien-ping Feng, J.Christopher Caylor, Bo Yu, Xiao Yan, Yi Ma, Xiaowei Wang, Dezhi Wang, Andrew Muto, Kenneth McEnaney, Matteo Chiesa, Zhifeng Ren and Gang Chen. High-performance flat-panel solar thermoelectric generators with high thermal concentration. Nature Materials 10, pp.532-538. May 1, 2011

${ }^{12}$ T. Sun, H. H. Hng, Q. Y. Yan, and J. Ma. Enhanced high temperature thermoelectric properties of $\mathrm{Bi}$-doped c-axis oriented $\mathrm{Ca}_{3} \mathrm{C}_{4} \mathrm{O}_{9}$ thin films by pulsed laser disposition. Journal of Applied Physics 108. AIP Publishing. October 21, 2008.

${ }^{13}$ Haoshan Hao, Qinglin He, ChangQing Chen, Hongqei Sun and Xing Hu. Textured structure and anisotropic thermoelectric properties of $\mathrm{Ca}_{2.7} \mathrm{Bi}_{0.3} \mathrm{C}_{04} \mathrm{O}_{9}$ oxide prepared by conventional solid-state reaction. International Journal of Modern Physics B Vol. 23, No.1. World Scientific Publishing Company. 2009.

${ }^{14}$ T. Takeuchi, T. Kondo, K. Soda, U. Mizutani, R. Funahashi, M. Shikano, S. Tsuda, T. Yokoya, S. Shin, T. Muro. Electronic structure and large thermoelectric power in 
$\mathrm{Ca}_{3} \mathrm{C}_{4} \mathrm{O}_{9}$. Journal of Electron Spectroscopy and Related Phenomena 137-140 pp.595599. March 18, 2004.

${ }^{15}$ S. Demirel, E. Altin, E. Oz, S. Altin, A. Bayri. An enhancement ZT and spin state transition of $\mathrm{Ca}_{3} \mathrm{C}_{4} \mathrm{O}_{9}$ with $\mathrm{Pb}$ doping. Journal of Alloys and Compounds 627 pp.430437. December 27, 2014.

${ }^{16}$ Susumu Fujii and Masato Yoshiya. Manipulating thermal conductivity by interfacial modification of misfit-layered cobaltites $\mathrm{Ca}_{3} \mathrm{C}_{4} \mathrm{O}_{9}$.

${ }^{17}$ Guangkun Ren, Jinle Lan, Chengcheng Zeng, Yaochin Liu, Bin Zhan, Sajid Butt, YuanHua Lin, and Ce-Wen Nan. High performance oxides-based thermoelectric materials. Journal of Electronic Materials, Vol. 67, No.1 pp. 211-221. 2014. 


\section{Chapter 3: \\ Effect of Bismuth Telluride Addition on the Electrical Performance of $\mathrm{Ca}_{3} \mathrm{CO}_{4} \mathrm{O}_{9}$ Ceramics}

\subsection{Background \& motivation of using Bismuth Telluride as a dopant in $\mathrm{Ca}_{3} \mathrm{Co}_{4} \mathrm{O}_{9}$}

Chalcogens, otherwise known as group 16 or the oxygen family, are considered among the main group elements. [1] They consist of the elements oxygen, sulfur, selenium, tellurium and polonium which can all be found in nature in both free and combined states. The name Chalcogen proposed by Wilhelm Blitz and colleague Werner Fischer in 1932 comes from the combination of the Greek word chalkos, meaning "bronze ore", and the Latinized Greek word genes, meaning born or produced. ${ }^{[1,2]}$ All of the Chalcogens have six valence electrons, leaving them two electrons short of a full outer shell. ${ }^{[2,3]}$ This means that they will most likely act as an accepter impurity when used as a dopant. Since they will accept electrons to fill their outer shell the baseline will lose two electrons leaving behind holes thus making it a good candidate for doping p-type material. Oxygen, sulfur and selenium are all nonmetals while tellurium is a metalloid meaning that its chemical properties are between those of a metal and a nonmetal which will make for a good semi conductive material. ${ }^{[1]}$ However, it is not certain whether polonium is a metal or a metalloid.

Tellurium, a member of the Chalcogen family was discovered in the Habsburg Empire in 1782 by Franz-Joseph Müller von Reichenstein in a mineral containing tellurium and gold. ${ }^{[2,3]}$ His first thought was that it was already among the known elements mistaking it 
for antimony. In 1798, almost two decades later, Reichenstein sent a sample to German chemist Martin Heinrich Klaproth to analyze further. ${ }^{[3]}$ Klaproth discovered that it was in fact a new element giving all credit to its discoverer von Reichenstein. ${ }^{[2]}$ Klaproth named this new element tellurium after the Latin word, tellus, meaning "earth". ${ }^{[2]}$ This was due to the ubiquitous amounts found in the earth's crust. It is known now that tellurium seldom occurs in its pure state and is often found as a compound in ores of gold along with silver, copper, lead, mercury or bismuth. ${ }^{[1,2]}$ Commercially, tellurium has mainly been used in alloys. It is often found in steel for its malleability, copper to improve machinability, and lead to resist vibrations and fatigue. ${ }^{[2,3]}$ Depending on its atomic alignment, tellurium is a semiconductor that can display a high electrical conductivity, which can be useful for thermoelectric applications.

As mentioned, tellurium combines easily with certain elements. It is also easy to engineer the band structure of a Chalcogen by impurity doping. ${ }^{[3]}$ If the Chalcogen has energy levels resonant with the host band this will alter the electrical properties. This chapter discusses the results of $\mathrm{Ca}_{3} \mathrm{Co}_{4} \mathrm{O}_{9}$ doped with the compound bismuth telluride, $\mathrm{Bi}_{2} \mathrm{Te}_{3}$. Bismuth telluride's small band gap of $0.16 \mathrm{eV}$ causes it to be a partially degenerate semiconductor with its Fermi-level close to the conduction band. ${ }^{[4]}$ This means that it has a high intrinsic carrier concentration which should reduce the electrical resistivity. Tellurium bonds with bismuth to comprise some of the best room temperature thermoelectric results with a temperature-independent ZT between 0.8 and 1.0. ${ }^{[4]}$

Weon Ho Shin et al reported a ZT value of 1.16 at $393 \mathrm{~K}$ with reduced graphene oxide, $\mathrm{RGO}$, incorporated $\mathrm{Bi}_{0.36} \mathrm{Sb}_{1.64} \mathrm{Te}_{3}$ composites which is $\sim 15 \%$ higher than its pristine form. ${ }^{[5]} \mathrm{Kim}$ et al. also reported an impressively high $\mathrm{ZT}$ of 1.86 at $320 \mathrm{~K}$ for $\mathrm{Bi}_{0.5} \mathrm{Sb}_{1.5} \mathrm{Te}_{3}$ due 
to a low lattice thermal conductivity of $0.33 \mathrm{~W} / \mathrm{mK}$. ${ }^{[6]}$ Cheng et al. report a ZT of 0.73 at $320 \mathrm{~K}$ for $\mathrm{Bi}_{2} \mathrm{Te}_{3}$ heteronanojunctions. ${ }^{[7]}$

\subsection{Experimental Procedure}

$\mathrm{Ca}_{3} \mathrm{Co}_{4} \mathrm{O}_{9}$ powders were obtained through a sol-gel route in which stoichiometric ratios of $\mathrm{Ca}\left(\mathrm{NO}_{3}\right)_{2} \bullet 4 \mathrm{H}_{2} \mathrm{O}, \mathrm{Co}\left(\mathrm{NO}_{3}\right)_{2} \cdot 6 \mathrm{H}_{2} \mathrm{O}$ were mixed in deionized water. Ethylene glycol and polyethylene glycol were used to aid polymerization of the solution and to vary particle size. ${ }^{[8]}$ Lastly in the process nitric acid was added under a fume hood to induce nitrate salts decomposition and facilitate new compound formation. ${ }^{[9]}$ The liquid solution was then submerged in a silicone oil bath on a hot plate and mechanically stirred at $353 \mathrm{~K}$ for 3 hours to achieve the sol-gel state. The sol-gel was distributed in to ceramic crucibles loosely covered with aluminum foil and put into a box furnace. The furnace was heated with a ramp rate of $10 \mathrm{~K}$ per minute reaching a temperature of $773 \mathrm{~K}$ where it was held for 2 hours. Holding the temperature here, at $773 \mathrm{~K}$, for the allotted time ensures that any inorganic material is removed from the material. The furnace was then cooled at a rate of $10 \mathrm{~K}$ per minute. The ashes were suspended in ethanol and ball milled for 20 minutes alternating between 1 minute of milling and 1 minute of resting in order to aid in the dissipation of heat. The ball milled powders were calcined in an oxygen rich induced environment with a ramp rate of $10 \mathrm{~K}$ per minute reaching a temperature of $973 \mathrm{~K}$ and held for 4 hours to achieve the $\mathrm{Ca}_{3} \mathrm{C}_{4} \mathrm{O}_{9}$ crystal structure. The powders were then cooled down to room temperature at $10 \mathrm{~K}$ per minute. $\mathrm{Bi}_{2} \mathrm{Te}_{3}$ was then added in nonstoichiometric ratios to the $\mathrm{Ca}_{3} \mathrm{C}_{4} \mathrm{O}_{9}$ crystal. The two powders were hand ground 
together for uniformity then placed in the ball mill suspended in ethanol for another 20 minutes in 1 minute intervals then set out to dry.

Two pellets were pressed, one to test its electrical properties and the other for its thermal properties. The calcined powders were pressed uniaxially with $1 \mathrm{GPa}$ of force and held for 10 minutes at $298 \mathrm{~K}$. The pellets' densities were obtained before they were put in to a tubular furnace to be sintered. The sintering process removes any extra moisture the pellet may contain and assures proper grain alignment. Pellets were sintered in an oxygen rich induced environment with a ramp rate of $10 \mathrm{~K}$ per minute and held at $1233 \mathrm{~K}$ for 9 hours. They were then cooled back to room temperature at a rate of $4 \mathrm{~K}$ per minute and once again the densities were taken. Pellets were cut into a $2 \mathrm{~mm} \times 3.5 \mathrm{~mm}$ x $9 \mathrm{~mm}$ rectangular shapes and inserted into a Linseis LSR 3-Seebeck machine, subjected to a low pressure helium, He, environment where the pellet's electrical resistivity and Absolute Seebeck Coefficient were simultaneously determined by means of a dc four-probe method. Since helium is an inert gas it will not react or interfere with the measurement. A second pellet was cut along a different axis and inserted into the Linseis LFA 1000 machine to test its thermal conductivity. The pellet was cut to be $2 \mathrm{~mm}$ thick and polished to a diameter of $11-13 \mathrm{~mm}$. Any less than $11 \mathrm{~mm}$ and there would be room for heat to escape the holder, any more than $13 \mathrm{~mm}$ and the pellet would be too large for the holder. The thermal conductivity is calculated by the equation

$$
\mathrm{K}=\lambda \mathrm{C}_{\mathrm{p}} \rho_{\mathrm{m}}
$$

Where $\lambda$ is the thermal diffusity in meters squared per second $\left(\mathrm{m}^{2} / \mathrm{s}\right), C_{p}$ is the specific heat capacity in joules per kilogram kelvin $(\mathrm{J} / \mathrm{kg} \cdot \mathrm{K})$, and $\rho_{m}$ is the mass density in grams 
per centimeter cubed $\left(\mathrm{g} / \mathrm{cm}^{3}\right)$. As stated in the first section, these are the three values needed in order to obtain the materials' ZT value. For this set the concentrations of $\mathrm{Bi}_{2} \mathrm{Te}_{3}$ were as follows: $\mathrm{Ca}_{3}\left(\mathrm{Bi}_{2} \mathrm{Te}_{3}\right)_{\mathrm{u}} \mathrm{Co}_{4} \mathrm{O}_{9}$ where $\mathrm{u}=0.0,0.003,0.006,0.025,0.05$, and 0.1.

\subsection{Electrical Performance of $\mathrm{Ca}_{3} \mathrm{Co}_{4} \mathrm{O}_{9}$ doped with $\mathrm{Bi}_{2} \mathrm{Te}_{3}$}

Each doping concentration had an electrical resistivity that was higher than the baseline; up to $\sim 65 \%$ higher. This is not caused by over doping considering there were samples with a very low concentration. It also goes to show that it's not the case where the sample was under doped either considering the highest concentration had a resistivity almost 7 times that of the baseline. This chemistry set does, however, reinforce the intimate connection between the resistivity and the Seebeck coefficient through the carrier concentration. As mentioned in the literature review a decrease in carrier concentration generally leads to an increase in resistivity. This can clearly be seen in the $\mathrm{Ca}_{3} \mathrm{Co}_{4} \mathrm{O}_{9}$ sample doped with $\mathrm{Bi}_{2} \mathrm{Te}_{3} 0.1$ in figure 15. This reduction in carrier concentration then leads to a slight enhancement of the Seebeck coefficient in the same sample which can be seen in figure 16. 


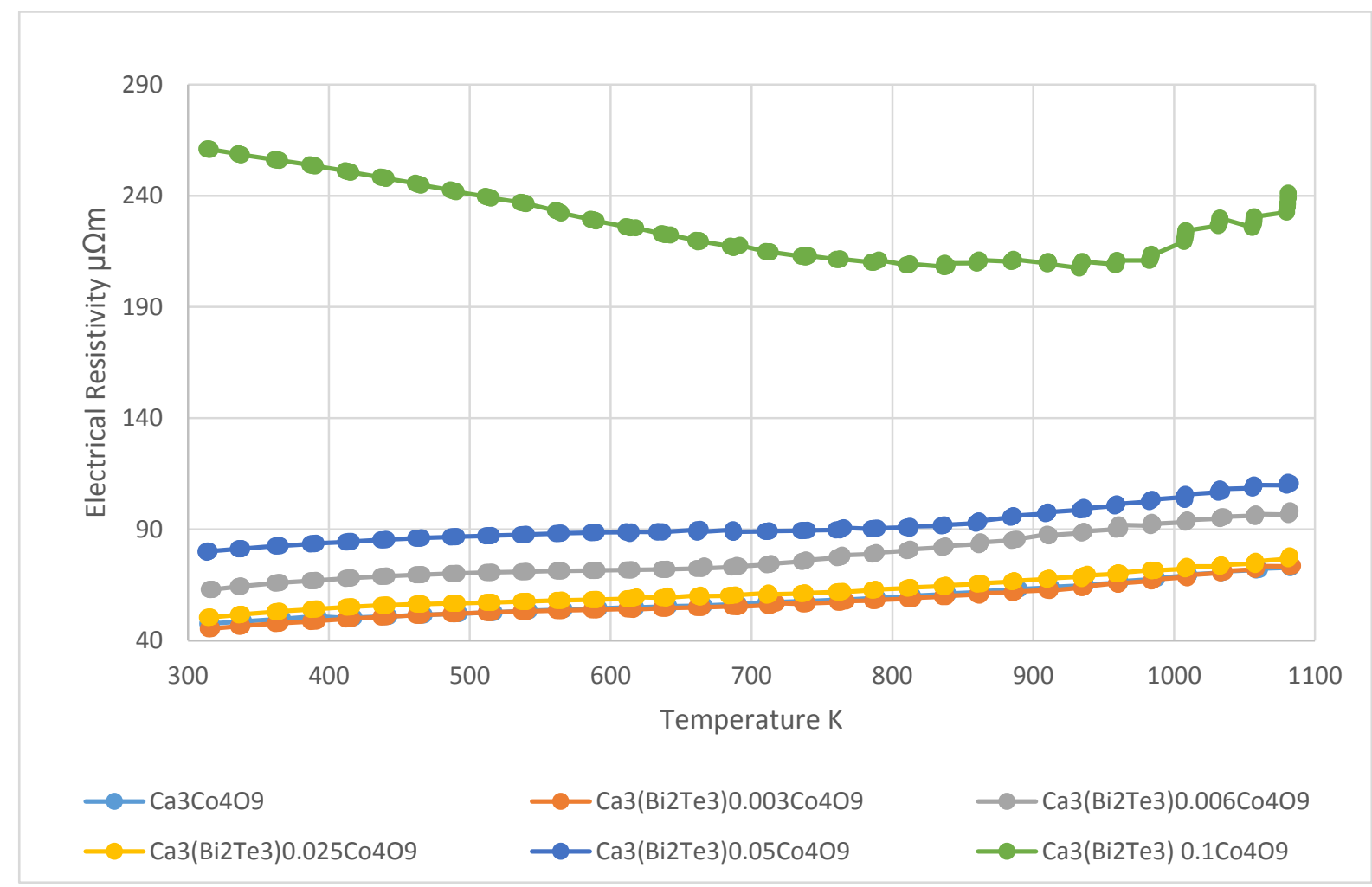

Figure 15 Temperature dependence of electrical resistivity for $\mathrm{Ca}_{3} \mathrm{Co}_{4} \mathrm{O}_{9} \mathrm{Bi}_{2} \mathrm{Te}_{3}$ addition.

It can be observed from figure 16 that there is in fact a benefit to the doping of $\mathrm{Bi}_{2} \mathrm{Te}_{3}$ in the $\mathrm{Ca}_{3} \mathrm{Co}_{4} \mathrm{O}_{9}$. At $315 \mathrm{~K}$ the Seebeck coefficient experiences a large increase up to $\sim 160 \mu \mathrm{V} / \mathrm{K}$ which is a $20 \mu \mathrm{V} / \mathrm{K}$ jump. Generally the Seebeck coefficient is highly intrinsic to the particular material and $\mathrm{Ca}_{3} \mathrm{Co}_{4} \mathrm{O}_{9}$ has a very stable and consistent room temperature Seebeck coefficient of around $140 \mu \mathrm{V} / \mathrm{K}$. More often than not any variation of the Seebeck coefficient manifests itself as an increase or decrease as the temperature rises. A change in Seebeck coefficient at room temperature is not a common occurrence. This enhancement in the Seebeck coefficient is most likely the direct cause of the $\mathrm{Bi}_{2} \mathrm{Te}_{3}$ addition. Due to the increase in resistivity along with the lack of change in Seebeck 
coefficient, with the exception for the Seebeck coefficient of the $\mathrm{Bi}_{2} \mathrm{Te}_{3} 0.1$ sample, the power factor decreases significantly shown in figure 17 . The resistivities of the samples were too high to offset the room temperature enhancement in the Seebeck coefficient. This caused all samples to achieve a lower power factor than the pure $\mathrm{Ca}_{3} \mathrm{Co}_{4} \mathrm{O}_{9}$.

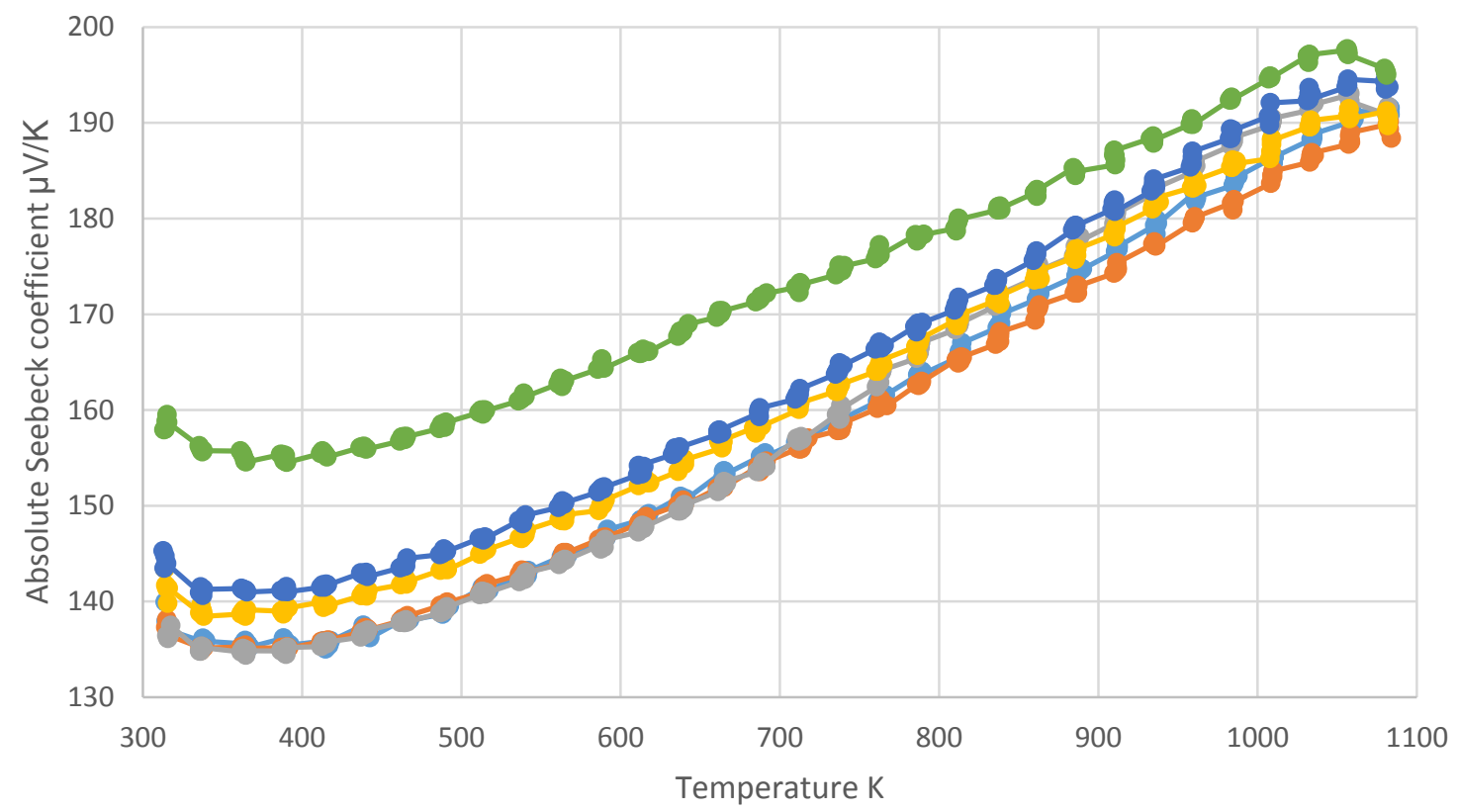

Figure 16 Temperature dependence of absolute seebeck coefficient for $\mathrm{Ca}_{3} \mathrm{Co}_{4} \mathrm{O}_{9}$ $\mathrm{Bi}_{2} \mathrm{Te}_{3}$ addition. 


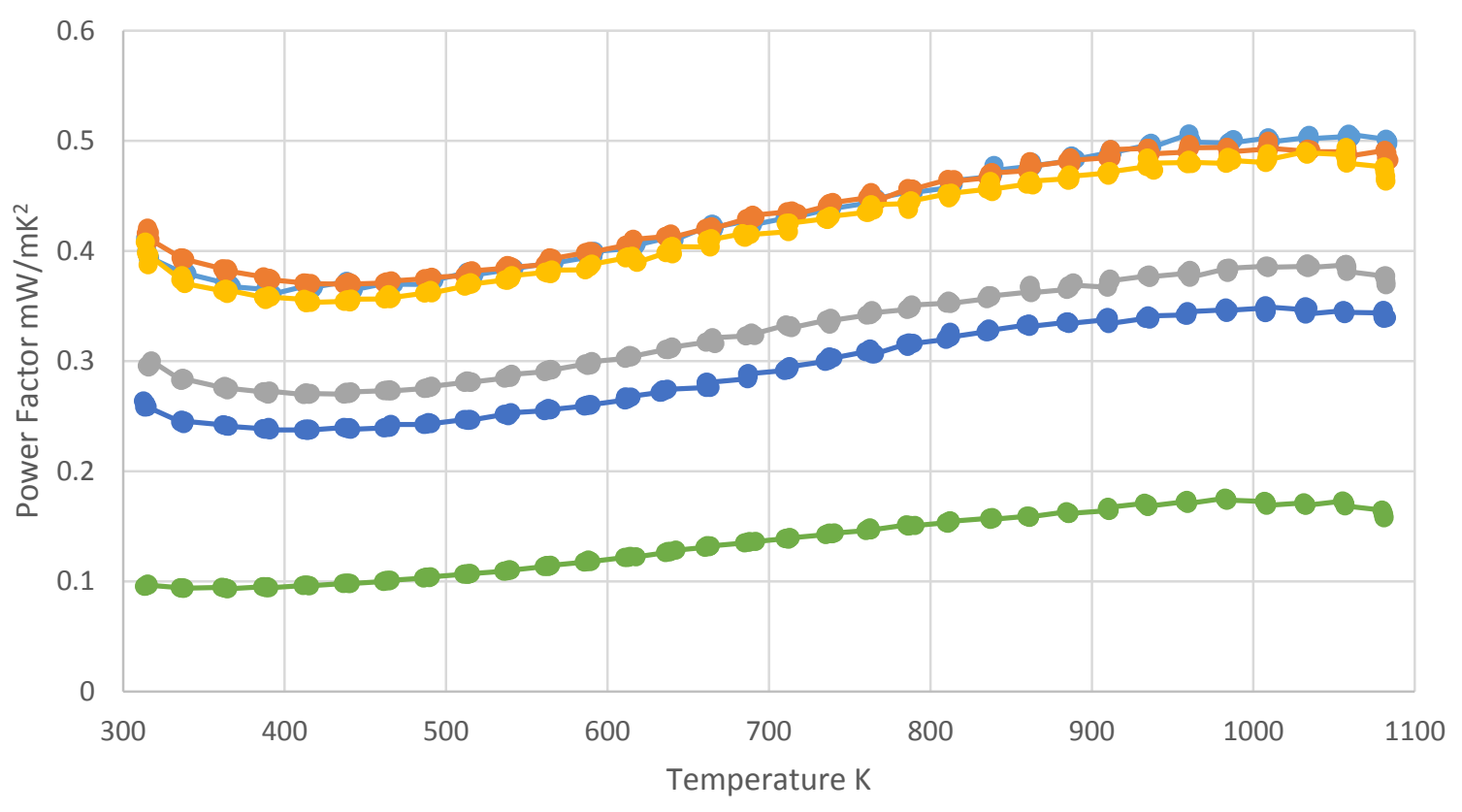

$\longrightarrow$ Са3С0409

$\longrightarrow$ - Ca3(Bi2Te3)0.003Co4O9

$\longrightarrow \mathrm{Ca3}(\mathrm{Bi} 2 \mathrm{Te} 3) 0.006 \mathrm{Co} 04 \mathrm{O}$

$\longrightarrow \mathrm{Ca3}(\mathrm{Bi} 2 \mathrm{Te} 3) 0.025 \mathrm{Co} 409$

$\rightarrow$ Ca3(Bi2Te3)0.05Co4O9

$\longrightarrow \mathrm{Ca3}(\mathrm{Bi2Te})$ 0.1Co409

Figure 17 Temperature dependence of power factor for $\mathrm{Ca}_{3} \mathrm{Co}_{4} \mathrm{O}_{9} \mathrm{Bi}_{2} \mathrm{Te}_{3}$ addition.

\subsection{Summary of results for $\mathrm{Bi}_{2} \mathrm{Te}_{3}$ doped $\mathrm{Ca}_{3} \mathrm{Co}_{4} \mathrm{O}_{9}$}

The literature has shown great difficulty in enhancing the Absolute Seebeck coefficient and although bismuth telluride has been reported on extensively throughout the literature this is the first time this enhancement in Absolute Seebeck Coefficient at room temperature has been investigated. Bismuth telluride enhanced the Seebeck coefficient from $\sim 140 \mu \mathrm{V} / \mathrm{K}$ to $\sim 158 \mu \mathrm{V} / \mathrm{K}$ at $315 \mathrm{~K}$. This shows that a large room temperature Seebeck coefficient can be achieved through doping the $\mathrm{Ca}_{3} \mathrm{Co}_{4} \mathrm{O}_{9}$ ceramic with $\mathrm{Bi}_{2} \mathrm{Te}_{3}$. There is also hope in experiencing this enhancement in the Absolute Seebeck coefficient while tuning the carrier mobility to achieve a low electrical resistivity. Achieving 
a low electrical resistivity coupled with the enhanced Absolute Seebeck coefficient would then lead to a high power factor. This also opens up the possibility of optimizing the dopant in order to enhance the Absolute Seebeck coefficient. Determining whether the presence of the bismuth or the telluride lead to the enhancement can be achieved by isolating the dopants individually. To further investigate this Seebeck phenomenon at room temperature bismuth was used to dope the $\mathrm{Ca}_{3} \mathrm{C}_{4} \mathrm{O}_{9}$ ceramic and is detailed in chapter 4. Bismuth's micro- and nanostructures were analyzed to understand what was responsible for this enhancement in Absolute Seebeck coefficient. 


\subsection{References}

1 The Elements of Group 16. Chemistry LibreTexts. UCDavis. University of California. 2017.

2 Gagnon, Steve. It's Elemental: The Periodic Table of Elements. The Element Tellurium. Jefferson Lab. 2017

3 Guangkun Ren, Jinle Lan, Chengcheng Zeng, Yaochun Liu, Bin Zhan, Sajid Butt, YuanHua Lin, and Ce-Wen Nan. High performance oxides-based thermoelectric materials. Journal of the Minerals, Metals \& Materials Society. Vol.67, No.1, pp. 211-221, 2015.

4 Dambi Park, Sungjin Park, Kwangsik Jeong, Hong-Sik Jeong, Jea Yong Song \& MannHo Cho. Thermal and Electrical Conduction of Single-crystal $\mathrm{Bi}_{2} \mathrm{Te}_{3}$ Nanostructures gown using a one step process. Nature Scientific Reports. August 2015.

5 Weon Ho Shin, Kyunghan Ahn, Mahn Jeong, Jeong Seop Yoon, Jae min Song, Soonil Lee, Won Seon Seo, Young Soo Lim. Enhanced thermoelectric performance of reduced graphene oxide incorporated bismuth-antimony-telluride by lattice thermal conductivity reduction. Journal of Alloys and Compounds. Vol.718, pp.342-348, 2017.

6 Sang II Kim, Kyu Hyoung Lee, Hyoun A Mun, Hyun Sik Kim, Sung Woo Hwang, Jong Wook Roh, Dae Jin Yang, Weon Ho Shin, Xiang Shu Li, Young Hee Lee, G. Jeffrey Snyder, Sung Wng Kim. Dense dislocation arrays embedded in grain boundaries for high-performance bulk thermoelectrics. Science. Vol. 348, Issue 6230, pp. 109-114, 2015.

7 Lina Cheng, Zhi-Gang Chen, Lei Yang, Guang Han, Hong-Yi Xu, G. Jeffrey Snyder, Gao-Qing Lu, and Jin Zou. T-Shaped $\mathrm{Bi}_{2} \mathrm{Te}_{3}-\mathrm{Te}$ Heteronanojunctions: Epitaxial Growth, Structural Modeling, and Thermoelectric Properties. The Journal of Physical Chemistry. Vol. 117, pp. 12458-12464, 2013.

8 Marat Gunes, Macit Ozenbas. Effect of grain size and porosity on phonon scattering enhancement of $\mathrm{Ca}_{3} \mathrm{C}_{4} \mathrm{O}_{9}$. Journal of Alloys and Compounds Vol.626, pp.360-367, 2015.

9 Boyle, Cullen; Calvillo, Paulo; Chen, Yun; Barbero, Ever J.; Mclntyre, Dustin. Grain boundary segregation and thermoelectric performance enhancement of bismuth doped calcium cobaltite. Journal of the European Ceramic Society 36(3):601-607 Elsevier 2016 0955-2219

${ }^{10}$ Tao Wu, Trevor A. Tyson, Haiyan Chen, Jianming Bai, Hsin Wang and Cherno Jaye. A Structural Phase Transition in $\mathrm{Ca}_{3} \mathrm{Co}_{4} \mathrm{O}_{9}$ Associated with Enhanced High Temperature Thermoelectric Properties. American Physical Society. 2012

11 Quentin Lognoné, Franck Gascoin. On the effect of carbon nanotubes on the thermoelectric properties of $\mathrm{n}-\mathrm{Bi}_{2} \mathrm{Te}_{2.4} \mathrm{Se}_{0.6}$ made by mechanical alloying. Journal of Alloys and Compounds. 635, pp. 107-111, 2015.

${ }^{12}$ Yuzhuo Zhang, Hong Chen, Zhiliang Li, Ting Huang, Shuqi Zheng. Rational design and controlled synthesis of $\mathrm{Te}_{2} / \mathrm{Bi}_{2} \mathrm{Te}_{3}$ heterostructure nanostring composites. Journal of Crystal Growth. 421, pp. 13-18, 2015. 
${ }^{13}$ Michael P. Rowe, Li Qin Zhou, Debasish Banerjee, and Minjuan Zhang. Improvement of the thermoelectric figure-of-merit of a doped telluride nanocomposite by combining phonon scattering with grain boundary-modifying Zn-containing nanostructures. Journal of Electronic Materials, Vol.44, No.1, pp.425-430, 2015.

14 T. Sun, H. H. Hng, Q. Y. Yan, and J. Ma. Enhanced high temperature thermoelectric properties of $\mathrm{Bi}$-doped $\mathrm{c}$-axis oriented $\mathrm{Ca}_{3} \mathrm{C}_{4} \mathrm{O}_{9}$ thin films by pulsed laser deposition. AIP Journal of Applied Physics. 108, 083709. 2010.

15 Dabin Park, Hyun Ju, Jooheon Kim. Enhanced thermoelectric power factor and low thermal conductivity in one-dimensional $\mathrm{Te} / \mathrm{Ag}_{2} \mathrm{Te}$ composites. Ceramics International. Vol.43, pp. 11156-11162, 2017.

${ }^{16}$ M. Sabarinathan, M. Omprakash, S. Harish, M. Navaneethan, J. Archana, S. Ponnusamy, H. Ikeda, T. Takeuchi, C. Muthamizhchelvan, Y. Hayakawa. Enhancement of power factor by energy filtering effect in hierarchical $\mathrm{BiSbTe}_{3}$ nanostructures for thermoelectric applications. Applied Surface Science. Vol.418, pp.246-251, 2017.

${ }_{17}$ Zhi-Lei Wang, Takahiro Akao. Tetsuhiko Onda, Zhong-Chun Chen. Microstructure and thermoelectric properties of $\mathrm{Bi}-\mathrm{Sb}$-Te bulk materials fabricated from rapidly solidified powders. Scripta Materialia. Vol.136, pp.111-114, 2017.

18 Jaoshan Hao, Qinglin He, Changqing Chen, Hongwei Sun, and Xing Hu. Textured structure and anisotropic thermoelectric properties of $\mathrm{Ca}_{2.7} \mathrm{Bi}_{0.3} \mathrm{C}_{04} \mathrm{O}_{9}$ oxide prepared by conventional solid-state reaction. International Journal of Modern Physics B, Vol.23, No.1, pp.87-95, 2009.

${ }^{19}$ Kenji Tanabe, Ryuji Okazaki, Hiroki Taniguchi, and Ichiro Terasaki. Optical conductivity of layered calcium cobaltate $\mathrm{Ca}_{3} \mathrm{C}_{4} \mathrm{O}_{9}$. Journal of Physics: Condensed Matter. Vol.28, 2016. 


\section{Chapter 4: \\ Improving the TE performance and crystal texture of $\mathrm{Ca}_{3} \mathrm{Co}_{4} \mathrm{O}_{9}$ ceramics through Bismuth Cation Substitution}

\subsection{Introduction to Cation Substitution}

Ion substitution is the exchange of ions with similar size and charge ${ }^{[1]}$ Anion substitution is difficult due to how strongly bonded the anions are. This is the motivation for the extensive research into the use of cation substitution. The use of cation substitution has been reported on vastly in the literature. It is arguably the most common and successful approach to impurity doping. It is simple, straightforward, and achieves great results. The substitution method uses a concentration "x" of a specific dopant, in the case of this chapter, bismuth, that is then added into the of $\mathrm{Ca}_{3} \mathrm{C}_{4} \mathrm{O}_{9}$ baseline. By subtracting the same concentration from the Ca location two things happen. First, the stoichiometric ratio is preserved and secondly the dopant is forced to go into the lattice in place of the Ca vacancies. As mentioned this usually only works if the ionic radii and ionic charges are similar enough in size and charge.

\subsection{Background and motivation for Bismuth as a dopant in $\mathrm{Ca}_{3} \mathrm{Co}_{4} \mathrm{O}_{9}$}

Bismuth has an unusually low toxicity for being such a heavy metal which allows it to be commercially used in items such as cosmetics and medicine. ${ }^{[2]}$ This same stability and low toxicity means safer handling in the lab and in the environment. Bismuth is also 
considered the most naturally occurring diamagnetic element; more diamagnetic than any other metal. ${ }^{22]}$ Diamagnetism is a property of the material that allows it to create an induced magnetic field in an opposite direction to that of an externally applied magnetic field. [4] Superconductors are usually considered perfect diamagnets. This diamagnetic property gives bismuth the ability to have one of the lowest thermal conductivities of all metals. ${ }^{[4]}$ This is advantageous when calculating its figure of merit.

$\mathrm{Ca}_{3} \mathrm{C}_{4} \mathrm{O}_{9}$ ceramics doped with bismuth were shown to begin melting at sintering temperatures of $1273 \mathrm{~K}$; while, the undoped $\mathrm{Ca}_{3} \mathrm{Co}_{4} \mathrm{O}_{9}$ can be synthesized up to $1373 \mathrm{~K}$ demonstrating that bismuth may form a liquid phase aiding in the sliding of grains and densification of the material. Bismuth can then increase in size $3.32 \%$ upon solidification increasing lattice parameters and further helping to densify the bulk sample to improve grain connectivity and enhance carrier mobility thus reducing its electrical resistivity. ${ }^{[3,4,5]}$ Bismuth has been commonly shown to decrease electrical resistivity through this increased carrier mobility as well as enhancing its Seebeck coefficient. ${ }^{[6,7]}$ Increase in grain growth due to partial substitution of bismuth for calcium ions contributes largely to this increase in carrier mobility as well. ${ }^{[8]}$ Siwen at al. confirm this grain growth and report a dimensionless figure of merit of $\sim 0.2$ at $973 \mathrm{~K}$ for $\mathrm{Ca}_{2.5} \mathrm{Bi}_{0.5} \mathrm{Co}_{4} \mathrm{O}_{9}$, shown in figure 18 , through solid state reaction of powders in stoichiometric ratios and conventional pressing and sintering. ${ }^{[9]}$ Eight years after Siwen, Yuhen et al. reported a figure of merit of 0.25 at $973 \mathrm{~K}$ for bismuth doped $\mathrm{Ca}_{0.85} \mathrm{Bi}_{0.15} \mathrm{C}_{04} \mathrm{O}_{9}$ prepared through sol-gel precursor powders and spark plasma sintering (SPS). ${ }^{[7]}$ 


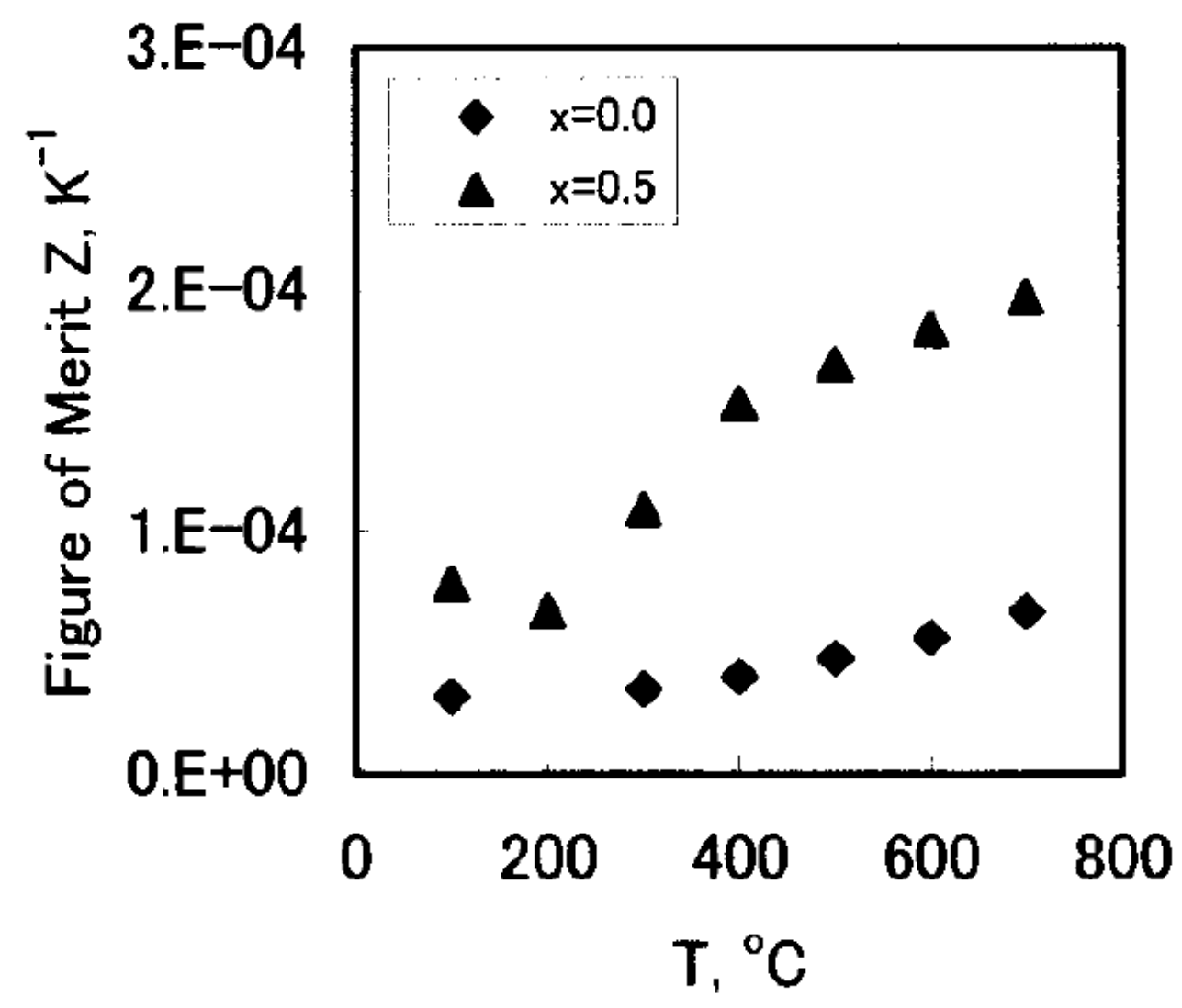

Figure 18 Temperature dependence of figure of merit for $\mathrm{Ca}_{3-x} \mathrm{Bi}_{x} \mathrm{Co}_{4} \mathrm{O}_{9+\delta}(x=0.0$ and 0.5) ${ }^{[9]}$

Bismuth substitution has also been effective in decreasing oxygen deficiencies in the system. ${ }^{[11]}$ Replacing divalent $\mathrm{Ca}^{2+}$ ions with trivalent $\mathrm{Bi}^{3+}$ ions in the $\mathrm{Ca}_{2} \mathrm{CoO}_{3}$ insulating layer causes bismuth to inject electrons in to the $\mathrm{CoO}_{2}$ conductive layer due to the charge imbalance. An increase in oxygen content is then experienced in order to stabilize this charge imbalance. This provides the hole carriers to the system, reducing the electrical resistivity. ${ }^{[11]}$ Since the hole carriers dominate the $\mathrm{Ca}_{3} \mathrm{C}_{4} \mathrm{O}_{9}$ system, this scenario contributes to a reduction in carrier density, $\mathrm{n}$, playing a large role in increasing the Seebeck coefficient as well. ${ }^{[11]}$ Furthermore, according to W. Koshibae et al. the 
substitution of $\mathrm{Bi}^{3+}$ is expected to lower the overall valence of cobalt, thus resulting in a decrease in $\mathrm{Co}^{4+}$ concentration in exchange for the $\mathrm{Co}^{3+}$ valence state in the $\mathrm{CoO}_{2}$ conductive layer to cause an enhancement of the Seebeck coefficient. [10]

Masashi et al. reported on the increase in cell volume of $\mathrm{Ca}_{3} \mathrm{C}_{4} \mathrm{O}_{9}$ with increased doping of bismuth, confirming that not only does bismuth promote grain growth but it also enters the lattice, upon doping, in place of calcium vacancies to increase the overall lattice parameters. ${ }^{[11]}$ By entering the lattice, bismuth stabilizes the crystal structure and eliminates stacking faults improving grain connectivity by moderating the misfit relationship between the insulating $\mathrm{Ca}_{2} \mathrm{CoO}_{3}$ and the conducting $\mathrm{CoO}_{2}$ layers. ${ }^{[11]}$ This improvement in grain connectivity causes a reduction to the electrical resistivity. Also, relaxing the misfit lattice can shorten the phonon mean free path. [11] This lattice relaxation, along with bismuth's size and mass, are all known to reduce its thermal conductivity, helping to contribute to a higher figure of merit. ${ }^{[6,7]}$ This lattice parameter increase is shown in figure 19. 

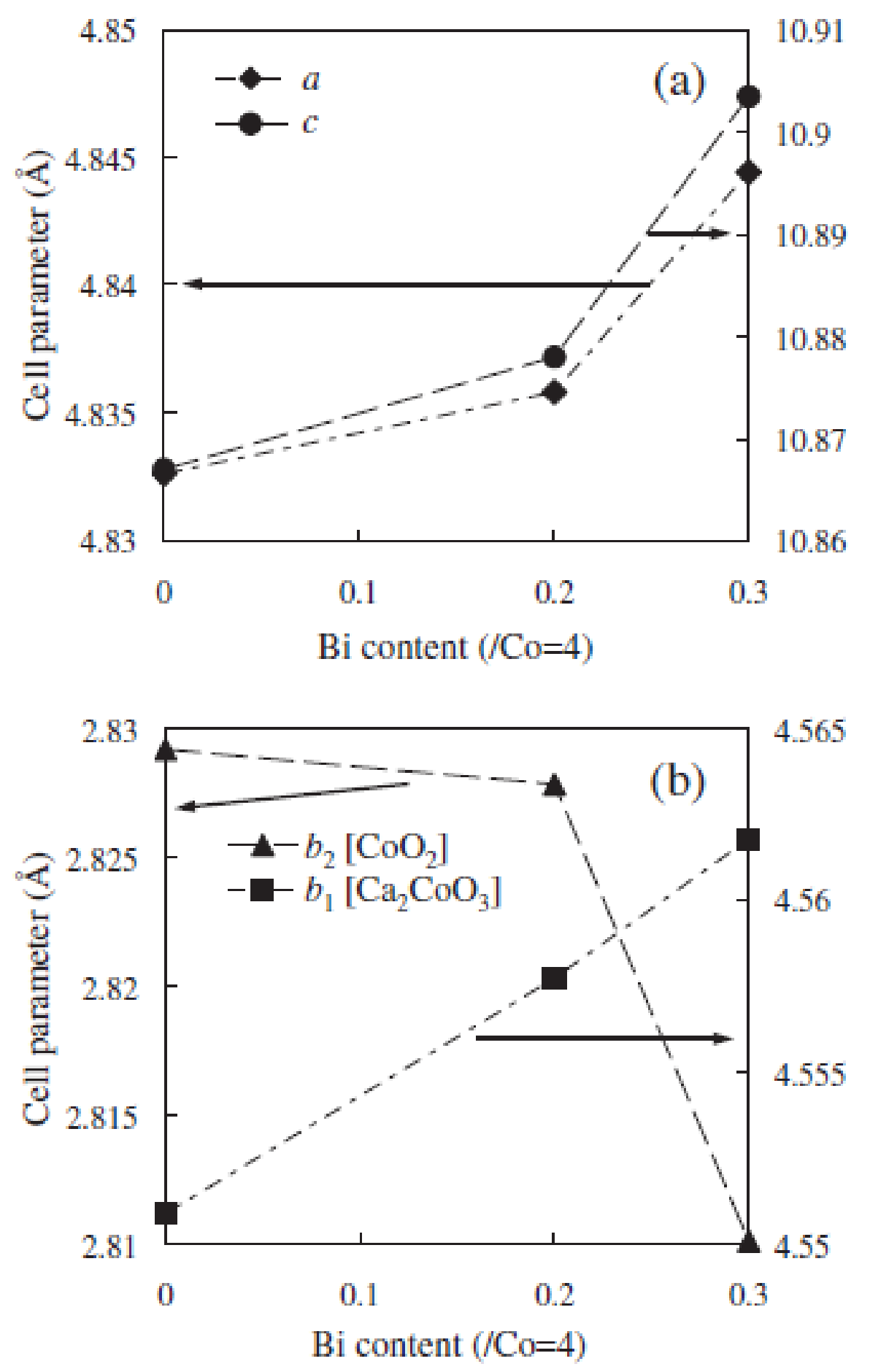

Figure 19 Lattice parameters calculated by Rietveld analysis as a function of $\mathrm{Bi}$ composition $b_{1}$ and $b_{2}$ correspond to the $b$ parameters of $\left[\mathrm{Ca}_{2} \mathrm{CoO}_{3}\right]$ and $\left[\mathrm{CoO}_{2}\right]$ layers, respectively. ${ }^{[11]}$ 
Reports on bismuth doped calcium cobaltite single crystal have shown to improve $\mathrm{Ca}_{3} \mathrm{Co}_{4} \mathrm{O}_{9}$ 's figure of merit to 0.9 at $973 \mathrm{~K}$. ${ }^{[12]}$ However, there is a lack of information on the doping of bismuth in polycrystalline bulk samples with the use of a sol-gel route with conventional pressing and sintering methods. There is also no study on the correlation between the microstructure and its properties.

This chapter focuses on the investigation into the possible Seebeck coefficient enhancement while achieving a low electrical resistivity and thermal conductivity with bismuth doping. X-ray diffraction patterns of single crystals prepared with partially bismuth substituted $\mathrm{Ca}_{3} \mathrm{Co}_{4} \mathrm{O}_{9}$ reported by Masashi et al. displayed a secondary phase of $\mathrm{Bi}_{2} \mathrm{Ca}_{2} \mathrm{Co}_{2} \mathrm{O}_{x}$ with the molar ratio of $\mathrm{Ca}: \mathrm{Bi}: \mathrm{Co}=2 \cdot 5: 0.5: 4 .{ }^{[11]}$ This secondary phase, most likely due to the over doping of bismuth, can cause an increase in electrical resistivity showing that a Ca:Bi ratio of 2.5:0.5 is most likely too large and proved to be a reference point when choosing the doping concentrations used in this chapter.

\subsection{Experimental Procedure}

$\mathrm{Ca}_{3-\mathrm{x}} \mathrm{Bi}_{x} \mathrm{C}_{4} \mathrm{O}_{9}$ powders where $\mathrm{x}=0.0,0.1,0.2,0.3,0.4$ were obtained through a solgel route in which stoichiometric ratios of $\mathrm{Ca}\left(\mathrm{NO}_{3}\right)_{2} \cdot 4 \mathrm{H}_{2} \mathrm{O}, \mathrm{Co}\left(\mathrm{NO}_{3}\right)_{2} \cdot 6 \mathrm{H}_{2} \mathrm{O}$ and $\mathrm{Bi}\left(\mathrm{NO}_{3}\right)_{2} \cdot 5 \mathrm{H}_{2} \mathrm{O}$ were mixed in deionized water. Ethylene glycol and polyethylene glycol were used to aid polymerization of the solution and vary particle size. Lastly in the process nitric acid was added to induce nitrate salts decomposition and facilitate new compound formation. ${ }^{[12]}$ The liquid solution was then submerged in a silicone oil bath on a hot plate and mechanically stirred at $353 \mathrm{~K}$ for 3 hours to achieve the sol-gel state. The sol-gel was then distributed to ceramic crucibles, loosely covered with aluminum foil, and put into a 
box furnace with a ramp rate of $10 \mathrm{~K}$ per minute reaching a temperature of $773 \mathrm{~K}$. The powders were held there for 2 hours before cooling back down to room temperature at 10 $\mathrm{K}$ per minute. Holding this temperature for the allotted time ensures that any inorganic material is removed from the material. The powders were deposited into a mortar where they were manually ground via pestle and mortar for 20 minutes. After the powders have been manually ground they were deposited in a tubular furnace where they were calcined in an oxygen rich induced environment at $973 \mathrm{~K}$ for 4 hours, with a ramp rate of $10 \mathrm{~K}$ per minute, to achieve the $\mathrm{Ca}_{3} \mathrm{Co}_{4} \mathrm{O}_{9}$ crystal lattice. The powders were then cooled down to room temperature at a rate of $10 \mathrm{~K}$ per minute.

Two pellets were pressed, one to test the electrical properties and the other for the thermal properties. The calcined powders were pressed uniaxially with $1 \mathrm{GPa}$ of force and held for 40 minutes at $423 \mathrm{~K}$ then allowed to cool back to $298 \mathrm{~K}$. The pellets' densities were obtained before they were put in to a tubular furnace to be sintered. The sintering process removes any extra moisture the pellet may contain and assures proper grain alignment. Pellets were sintered in an oxygen rich induced environment with a ramp rate of $10 \mathrm{~K}$ per minute and held at $1233 \mathrm{~K}$ for 9 hours. The pellets were then cooled back to room temperature at a rate of $4 \mathrm{~K}$ per minute and once again the densities were taken. Pellets were cut into a $2 \mathrm{~mm} \times 3.5 \mathrm{~mm}$ rectangular shapes and inserted into a Linseis LSR 3-Seebeck machine, subjected to a low pressure helium, He, environment where the pellet's electrical resistivity and Absolute Seebeck Coefficient were simultaneously determined by means of a dc four-probe method. Since helium is an inert gas it will not react or interfere with the measurement. A second pellet was cut along a different axis and inserted into the Linseis LFA 1000 machine to test its thermal conductivity. The pellet 
was cut to be $2 \mathrm{~mm}$ thick and polished to a diameter of $11-13 \mathrm{~mm}$. Any less than $11 \mathrm{~mm}$ and there would be room for heat to escape the holder, any more than $13 \mathrm{~mm}$ and the pellet would be too large for the holder. The thermal conductivity is calculated by the equation

$$
\mathrm{K}=\lambda \mathrm{C}_{\mathrm{p} \rho \mathrm{m}}
$$

Where $\lambda$ is the thermal diffusity in meters squared per second $\left(\mathrm{m}^{2} / \mathrm{s}\right), \mathrm{C}_{\mathrm{p}}$ is the specific heat capacity in joules per kilogram kelvin $(\mathrm{J} / \mathrm{kg} \cdot \mathrm{K})$, and $\rho_{\mathrm{m}}$ is the mass density in grams per centimeter cubed $\left(\mathrm{g} / \mathrm{cm}^{3}\right)$. As stated in the first section, these are the three values needed in order to obtain the materials' ZT value. For bismuth substitution five different chemistries were used. The first chemistry was the $\mathrm{Ca}_{3} \mathrm{C}_{4} \mathrm{O}_{9}$ baseline and the other four are dopings of bismuth in 0.1 gram increments; $\mathrm{Ca}_{3-\mathrm{x}} \mathrm{Bi}_{x} \mathrm{C}_{4} \mathrm{O}_{9}$ where $\mathrm{x}=0.1,0.2,0.3$, and 0.4 .

\subsection{Experimental Results of Bismuth doped $\mathrm{Ca}_{3} \mathrm{C}_{4} \mathrm{O}_{9}$ using cation substitution}

4.4.1 Presence of bismuth in the lattice \& at the grain boundary of $\mathrm{Ca}_{3} \mathrm{C}_{4} \mathrm{O}_{9}$ ceramics

As figure 20 shows, the undoped sample starts at $315 \mathrm{~K}$ with an electrical resistivity at around $70 \mu \Omega m$ and as the temperature increases to $1100 \mathrm{~K}$ so too does the resistivity, to about $100 \mu \Omega \mathrm{m}$. This is characteristic of how a metal would act, increased resistivity with increased temperature. As the doping concentration of bismuth is increased the electrical resistivity steadily decreases in all temperature ranges. Doping up to $x=0.3$ proves to enhance grain growth, increase its grain anisotropy, along with improving its crystal texture development. ${ }^{[12]}$ The optimal resistivity occurs with a doping concentration 
of $\mathrm{Bi} \mathrm{X}=0.2$ and $\mathrm{Bi} \mathrm{x}=0.3$ both achieving a resistivity of around $28 \mu \Omega \mathrm{m}$ at room temperature increasing to around $45 \mu \Omega m$ at $1073 \mathrm{~K}$. The electrical resistivity increases drastically with the doping of the $\mathrm{Bi} x=0.4$. It has risen more than three times that of the undoped sample. This demonstrates the over doping of bismuth at this concentration. At bismuth $\mathrm{x}=0.4$ the sample is over doped and experiences deterioration of its grain alignment. Its lack of grain alignment is mainly due to is vast grain growth in comparison with the optimal samples of bismuth $x-0.2$ and $x=0.3$. This misalignment is evident from its drastic increase in electrical resistivity.

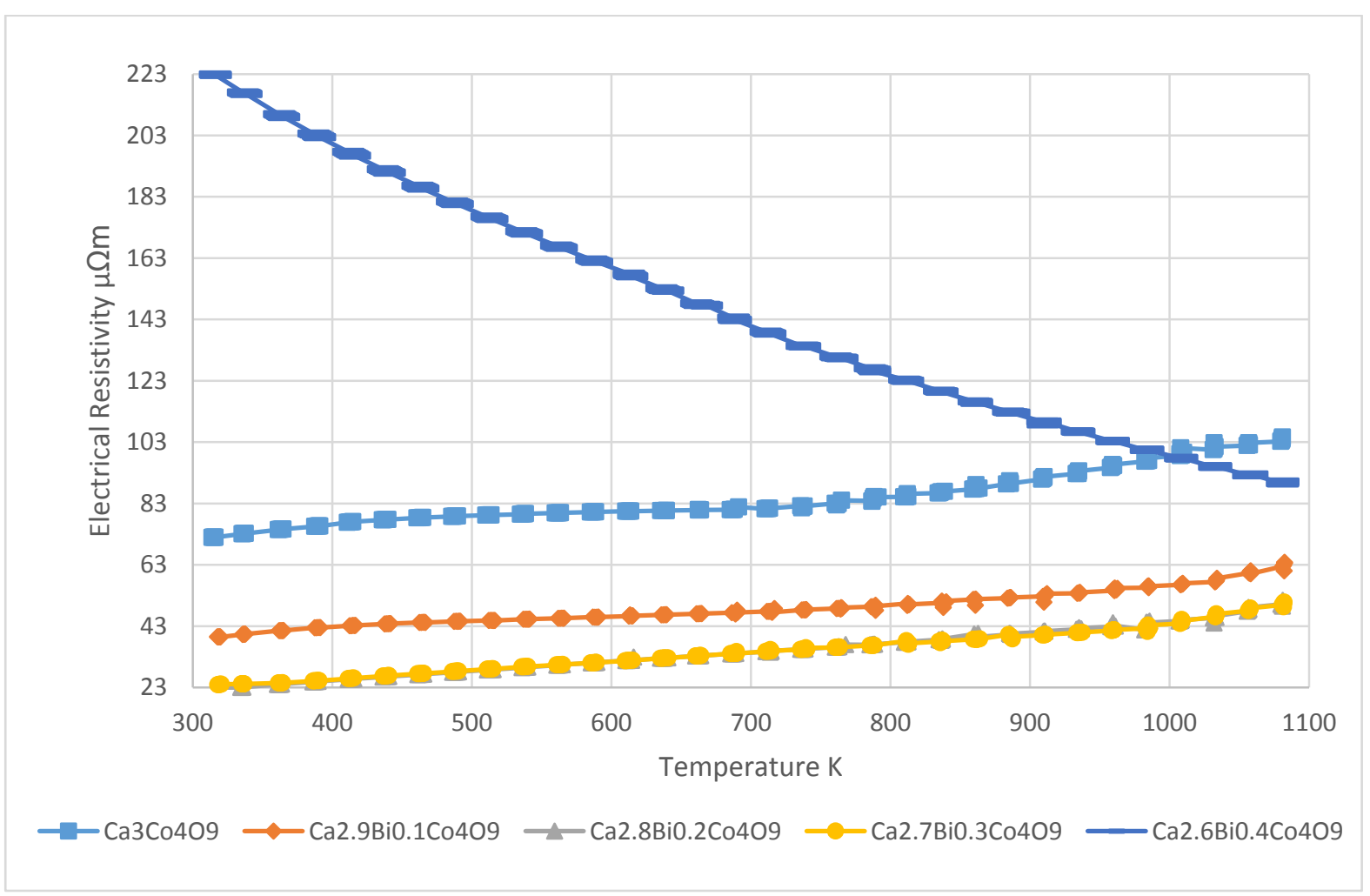

Figure 20 Temperature dependence of electrical resistivity for $\mathrm{Ca}_{3-x} \mathrm{Bi}_{x} \mathrm{Co}_{4} \mathrm{O}_{9}$ bismuth cation substitution. 
Figure 21 represents the Absolute Seebeck Coefficients of the different bismuth dopings. It can be seen that as soon as the bismuth is introduced, the room temperature Absolute Seebeck coefficient increases up 20-30 $\mu \mathrm{V} / \mathrm{K}$ from the undoped $\mathrm{Ca}_{3} \mathrm{Co}_{4} \mathrm{O}_{9}$ sample. This shows that the Seebeck coefficient enhancement experienced in the $\mathrm{Bi}_{2} \mathrm{Te}_{3}$ set from chapter 3 was most likely responsible due to the existence of the bismuth. With each increased concentration of Bismuth the room temperature Absolute Seebeck Coefficients are greater than the last. This trend stays consistent up until its saturation limit at around $150 \mu \mathrm{V} / \mathrm{K}$. As the temperature increases to $1073 \mathrm{~K}$ so do the Seebeck coefficients, with the exception of bismuth $x=0.4$ which succumbs to its large increase in carrier concentration at roughly $650 \mathrm{~K}$. This drop in Seebeck coefficient is highly indicative of an over doped sample. The grain growth was too vast causing improper alignment and drastically lowering carrier mobility in the sample. 


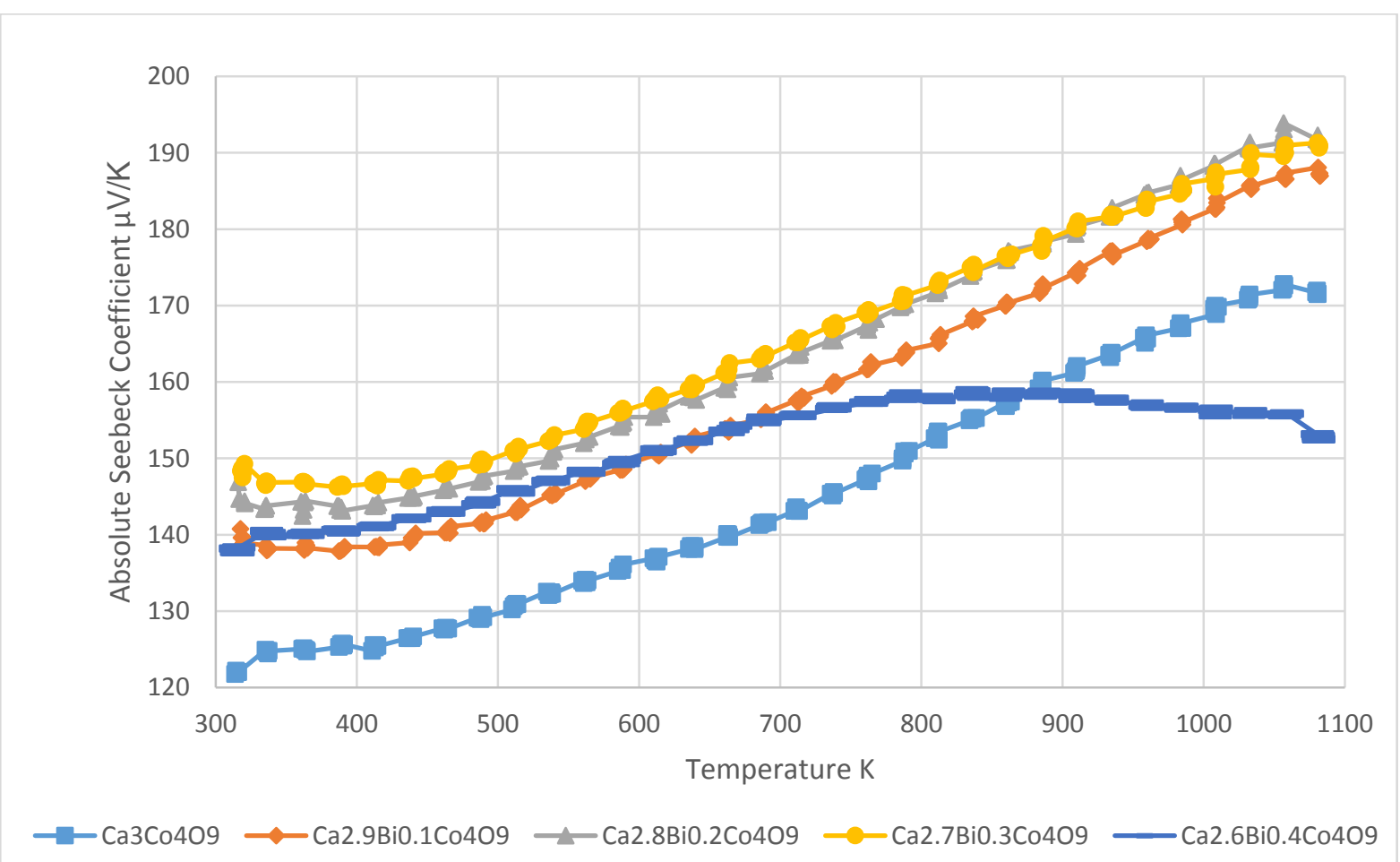

Figure 21 Temperature dependence of absolute seebeck coefficient for $\mathrm{Ca}_{3-x} \mathrm{Bi}_{x} \mathrm{Co}_{4} \mathrm{O}_{9}$ bismuth cation substitution.

4.4.2 Power factor enhancement of $\mathrm{Ca}_{3} \mathrm{CO}_{4} \mathrm{O}_{9}$ through Bismuth Substitution of $\mathrm{Ca}$

With increased doping the power factor shown in figure 22 increases as well. Both bismuth $\mathrm{x}=0.2$ and bismuth $\mathrm{x}=0.3$ start off with a very high power factor of over $0.90 \mathrm{~W} / \mathrm{mK}^{2}$ at room temperature which is more than four times greater than the power factor (PF) of the $\mathrm{Ca}_{3} \mathrm{Co}_{4} \mathrm{O}_{9}$ baseline sample. With a PF of $\sim 0.73 \mathrm{~W} / \mathrm{mK}^{2}$ at $1073 \mathrm{~K}$ it is almost three times higher than the $\mathrm{Ca}_{3} \mathrm{C}_{4} \mathrm{O}_{9}$ baseline. Bismuth $\mathrm{x}=0.4$ 's power factor however is lower than that of the baseline in all temperature ranges. This is a perfect example of the roles that the resistivity and Seebeck coefficient play in obtaining the power factor. The over doped sample of bismuth $\mathrm{x}=0.4$ has a very high resistivity at low temperatures which offsets its seemingly good Seebeck coefficient at the same temperature thus giving it a 
low power factor. Although the bismuth $\mathrm{x}=0.4$ resistivity drops consistently and significantly as the temperature increases, its Absolute Seebeck coefficient falls off drastically at $650 \mathrm{~K}$ being the major contributor for its poor power factor in the mid to high temperature range. In order to obtain a consistent high power factor, both values must yield good results throughout all temperatures ranges. This also explains the slight decrease in power factor of all samples as well, with the exception of the over doped sample. Even though all of their Absolute Seebeck coefficients steadily increase with increasing temperature their resistivities increase slightly as well causing almost a perfectly flat power factor. This rise is mainly due to the intrinsic nature of the resistivity of a metal in general. [2] The highest power factors are for the bismuth $x=0.2$ and $x=0.3$ samples, at $973 \mathrm{~K}$, with power factors of $\sim 0.80 \mathrm{~mW} / \mathrm{mK}^{2}$ and $\sim 0.83 \mathrm{~mW} / \mathrm{mK}^{2}$, respectively. Both are nearly three times that of the undoped sample and are also higher than any reportings for polycrystalline samples of $\mathrm{Ca}_{3} \mathrm{Co}_{4} \mathrm{O}_{9}$; comparable to that of the single crystal's power factor. [12] 


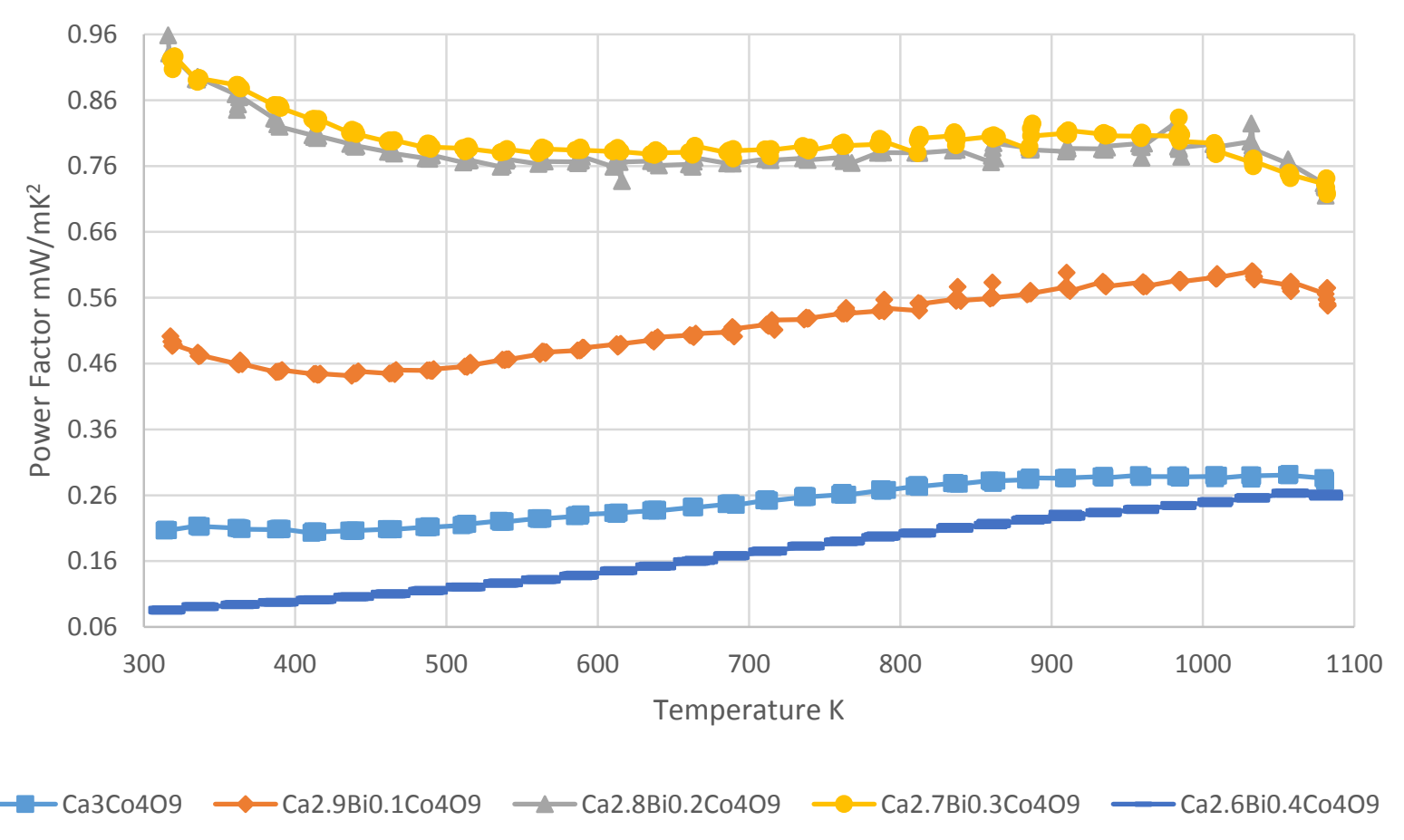

Figure 22 Temperature dependence of power factor for $\mathrm{Ca}_{3-x} \mathrm{Bi}_{x} \mathrm{Co}_{4} \mathrm{O}_{9}$ bismuth cation substitution.

\subsubsection{Microstructure of bismuth doped $\mathrm{Ca}_{3} \mathrm{C}_{4} \mathrm{O}_{9}$}

The alignment of grains can be seen in the Scanning Electron Microscope crosssectional images illustrated in figure 23 taken from the fractured surface of the pellets. Larger grains can make it more difficult for the grains to properly align themselves in a fashion that aids its electrical transport properties. Larger grains also mean less grain boundaries and less opportunity for phonon scattering. Figure 23 shows the increased improvement of the grain alignment with the increase in bismuth concentration from (a) to (d) representing $\mathrm{Ca}_{3} \mathrm{Co}_{4} \mathrm{O}_{9}, \mathrm{Ca}_{2.9} \mathrm{Bi}_{0.1} \mathrm{Co}_{4} \mathrm{O}_{9}, \mathrm{Ca}_{2.8} \mathrm{Bi}_{0.2} \mathrm{Co}_{4} \mathrm{O}_{9}$, and $\mathrm{Ca}_{2.7} \mathrm{Bi}_{0.3} \mathrm{Co}_{4} \mathrm{O}_{9}$, respectively. Image (e) and (f) show high and low magnification of the $\mathrm{Ca}_{2.6} \mathrm{Bi}_{0.4} \mathrm{C}_{4} \mathrm{O}_{9}$ over doped sample. There is vast grain growth and misalignment among the crystal 
texture of the sample. However, as mentioned, grain growth can have a positive effect on the results as well. Ruoming et al. claimed that $\mathrm{Ca}_{3} \mathrm{C}_{0} \mathrm{O}_{9}$ baseline has a Lotgering factor of about $\sim 0.74$ but after doping with bismuth they achieved a value of around 0.9. ${ }^{[13]}$ This is due to the grain growth and alignment caused by the doping of bismuth which significantly reduces the material's electrical resistivity. At the doping level of $\mathrm{Bi} x=0.4$ the sample becomes over doped and the grain growth becomes too significant for the grains to move or jitter as well during sintering in order to align themselves efficiently. This causes misalignment in the grains and does not allow for smooth transport of the electronhole pair causing a significant increase in the electrical resistivity which was shown in figure 20.
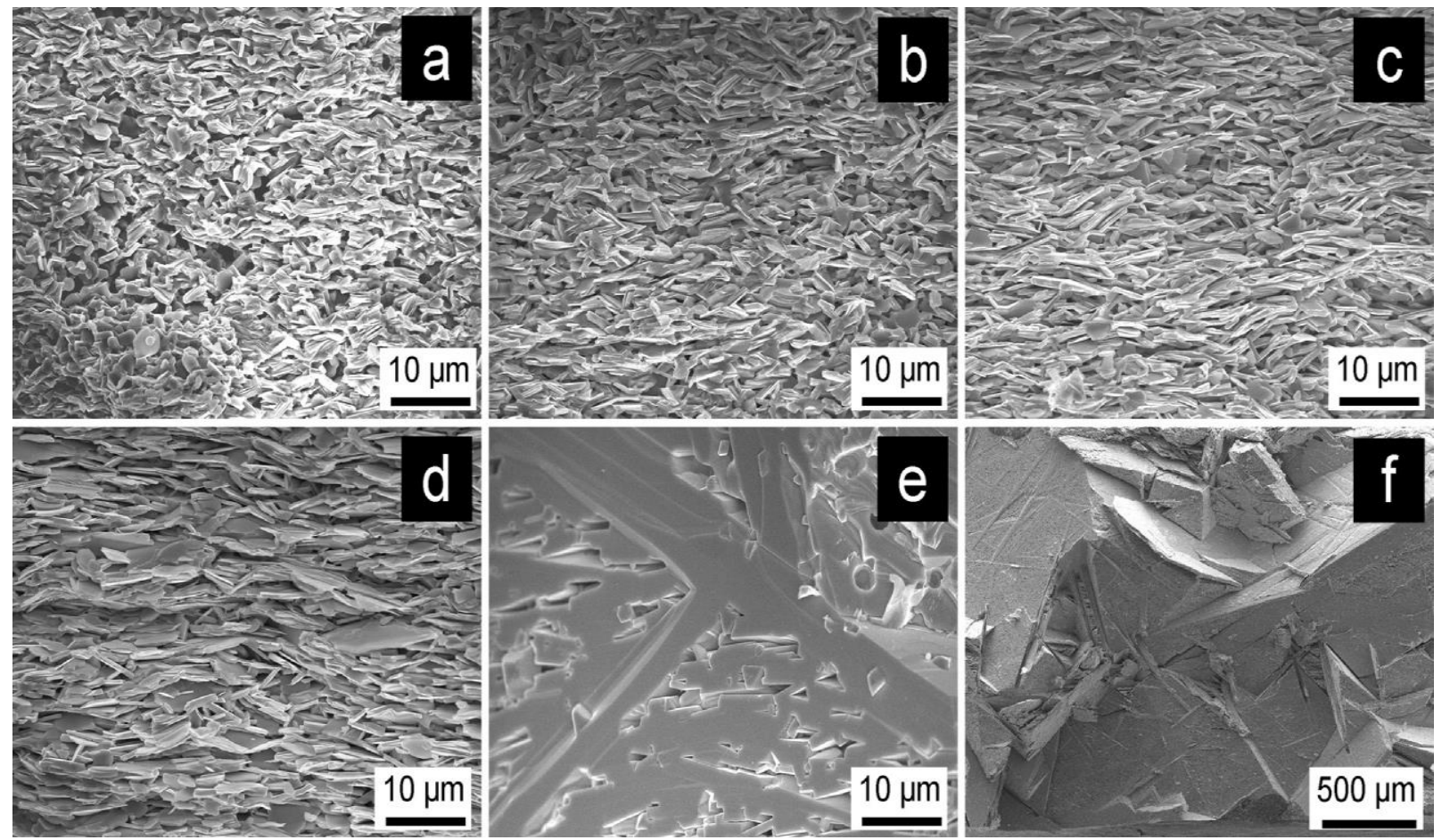

Figure 23 SEM cross-sectional view of undoped $\mathrm{Ca}_{3} \mathrm{Co}_{4} \mathrm{O}_{9}$ and doped $\mathrm{Ca}_{3-x} \mathrm{Bi}_{x} \mathrm{CO}_{4} \mathrm{O}_{9}$ with $x=0.0,0.1,0.2,0.3,0.4$. ${ }^{[12]}$ 


\subsubsection{Nanostructure of bismuth doped $\mathrm{Ca}_{3} \mathrm{Co}_{4} \mathrm{O}_{9}$}

Figure 24 shows the TEM nanostructure of the optimal sample of $\mathrm{Ca}_{2.7} \mathrm{Bi}_{0.3} \mathrm{C}_{0} \mathrm{O}_{9}$. Low magnification of the TEM diffraction contrast in image (a) shows a typical grain boundary and the adjacent two grains. The pink and green arrows labeled $C_{1}$ and $C_{2}$ respectively indicate the different c-axis orientations of these two grains with a misorientation angle of about $6^{\circ}$. Low magnification STEM Z-contrast from the same grain boundary is indicated in image (b). High magnification STEM Z-contrast of that same grain boundary shows two segregations sites in image (c). EDS point examinations were conducted at eight different locations and are summarized and illustrated in table 1. 


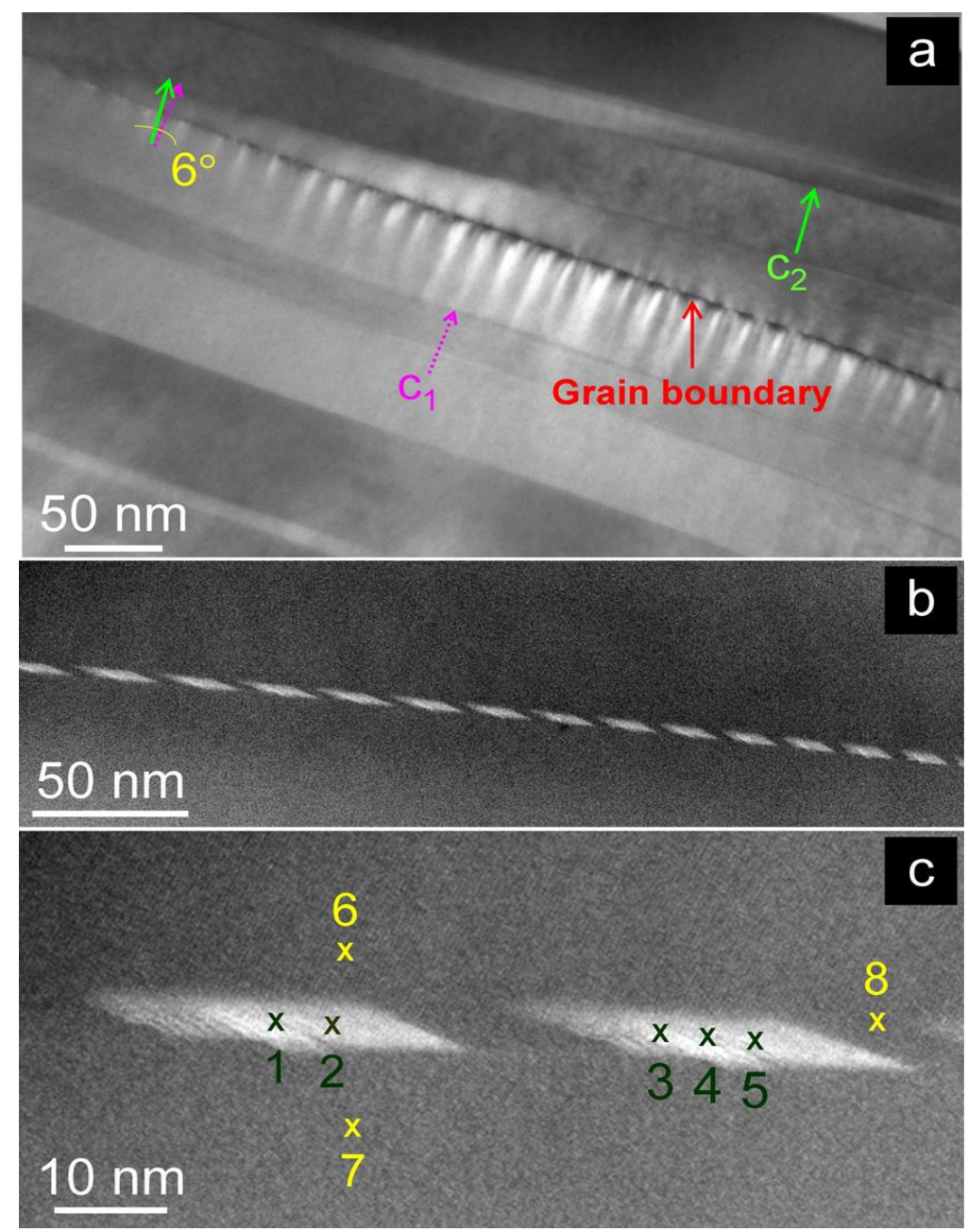

Figure 24 TEM nanostructure of $\mathrm{Ca}_{2.7} \mathrm{Bi}_{0.3} \mathrm{Co}_{4} \mathrm{O}_{9 .}{ }^{[12]}$

Table 1 EDS spot examination showing the chemistry of segregation regions and neighboring grains in the sample $\mathrm{Ca}_{2.7} \mathrm{Bi}_{0.3} \mathrm{CO}_{4} \mathrm{O}_{9 .}{ }^{[12]}$

\begin{tabular}{|l|r|r|r|r|r|r|r|c|}
\hline Spot & 1 & 2 & 3 & 4 & 5 & 6 & 7 & 8 \\
\hline $\mathrm{Ca}$ & 17.97 & 19.63 & 19.15 & 17.79 & 18.28 & 23.00 & 22.21 & 21.74 \\
$\mathrm{Co}$ & 21.42 & 22.49 & 23.34 & 24.30 & 26.12 & 22.31 & 24.26 & 22.61 \\
$\mathrm{O}$ & 54.25 & 53.08 & 50.94 & 51.33 & 50.11 & 52.54 & 51.99 & 54.13 \\
$\mathrm{Bi}$ & 6.36 & 4.80 & 6.58 & 6.59 & 5.48 & 2.15 & 1.53 & 1.52 \\
\hline
\end{tabular}


Figure 25 shows TEM images of undoped and bismuth doped $\mathrm{Ca}_{3} \mathrm{Co}_{4} \mathrm{O}_{9}$. Image (a) shows one $\mathrm{Ca}_{3} \mathrm{C}_{4} \mathrm{O}_{9}$ grain boundary and its two neighboring grains with nano-lamella morphologies. Image (b) shows the selected area diffraction pattern and indexing circled red in image (a). Image (c) shows the magnified grain boundary from the yellow dotted region framed in image (a). The nanostructure of $\mathrm{Ca}_{2.7} \mathrm{Bi}_{0.3} \mathrm{C}_{04} \mathrm{O}_{9}$ is shown in image (d).

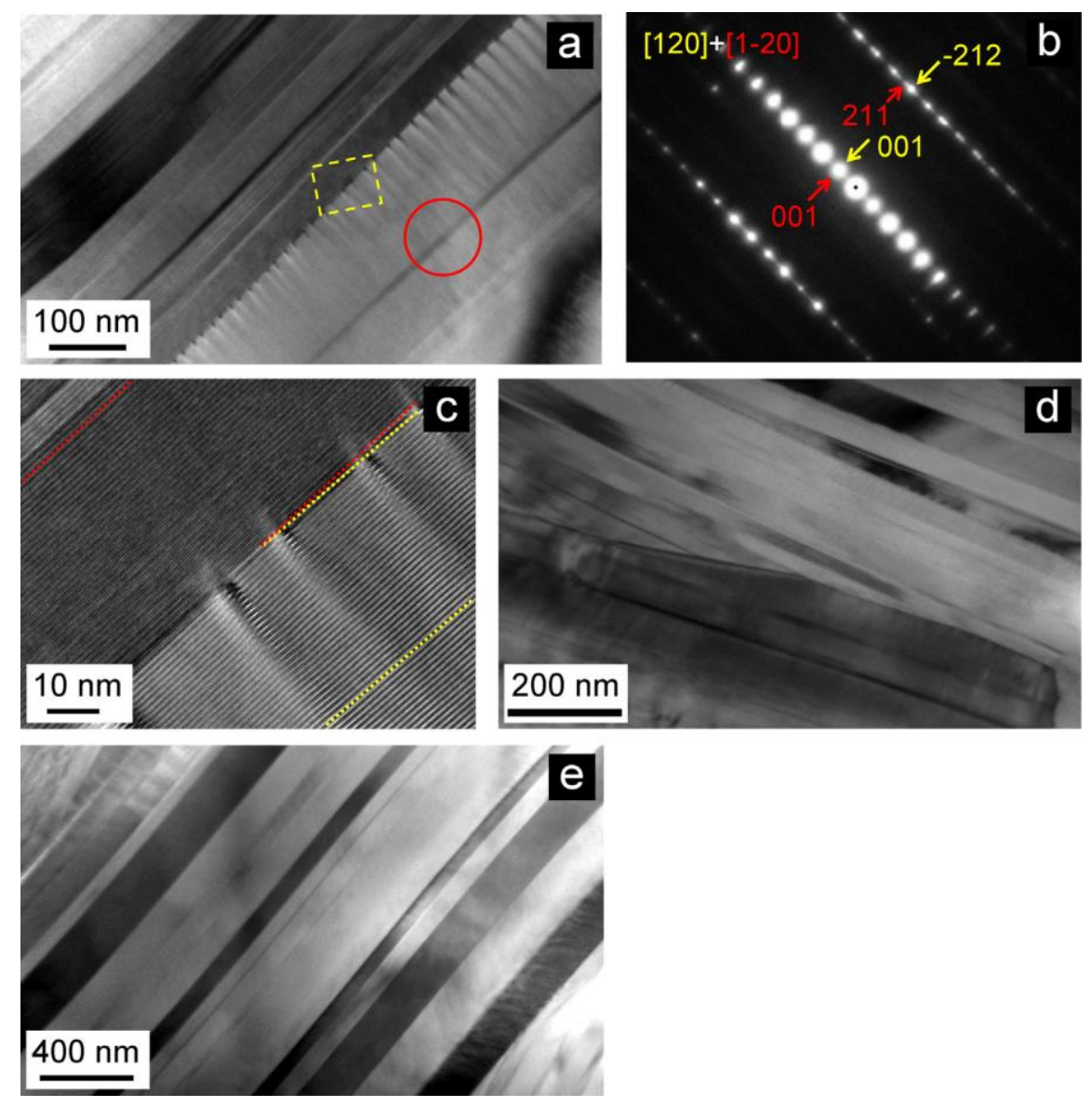

Figure 25 TEM images of undoped and bismuth doped $\mathrm{Ca}_{3} \mathrm{Co}_{4} \mathrm{O}_{9} .{ }^{[12]}$

\subsubsection{Impact of sample density on thermal conductivity}

The $\mathrm{Ca}_{3} \mathrm{Co}_{4} \mathrm{O}_{9}$ baseline has a thermal conductivity of $2.3 \mathrm{~W} / \mathrm{mK}$ at $373 \mathrm{~K}$ dropping to around $2 \mathrm{~W} / \mathrm{mK}$ at $1073 \mathrm{~K}$. It can be seen in figure 26 that upon doping with bismuth it 
immediately reduces the conductivity to $2.1 \mathrm{~W} / \mathrm{mK}$ at $373 \mathrm{~K}$ and drops below $1.9 \mathrm{~W} / \mathrm{mK}$ at $1073 \mathrm{~K}$. This is due to the increase in density at the grain boundary. Although bismuth mainly distributes itself directly into the grains it also deposits itself in the grain boundary causing its density to increase. With bismuth $x=0.3$ the thermal conductivity is slightly higher than that of the baseline. This is due to over doping and its grain growth which affected its figure of merit. As the grain size increases, the lattice thermal conductivity increases with it since larger grains mean fewer grain boundaries and weaker phonon interface scattering. ${ }^{[14]}$

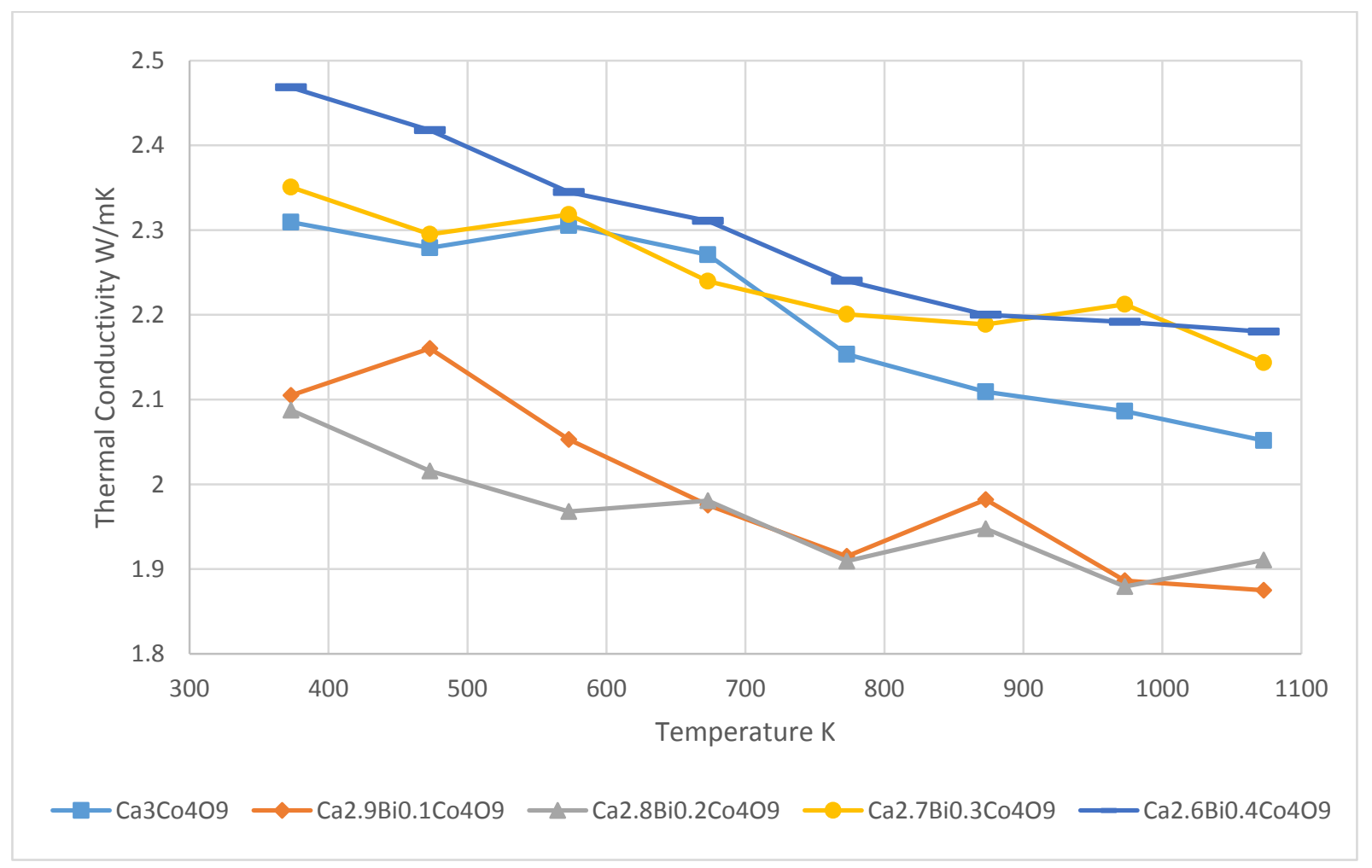

Figure 26 Temperature dependence for thermal conductivity of $\mathrm{Ca}_{3-x} \mathrm{Bi}_{x} \mathrm{CO}_{4} \mathrm{O}_{9}$ bismuth cation substitution. 
Bismuth $\mathrm{x}=0.4$ has the highest thermal conductivity due to its significant increase in density. As mentioned in equation 13 the pellet's density is directly related to the thermal conductivity and with a density of $4.80 \mathrm{~g} / \mathrm{cm}^{3}$ for the bismuth $\mathrm{x}=0.4$ sample this is much greater than the $3.70 \mathrm{~g} / \mathrm{cm}^{3}$ and $3.95 \mathrm{~g} / \mathrm{cm}^{3}$ of the bismuth $\mathrm{x}=0.1$ \& $\mathrm{x}=0.2$ respectively. The low densities of those specific samples reinforces the role that the sample's density plays in measuring its thermal conductivity.

\subsubsection{Significance of low thermal conductivity for achieving the high Figure of Merit}

The figure of merits of the undoped and bismuth doped $\mathrm{Ca}_{3} \mathrm{C}_{4} \mathrm{O}_{9}$ samples are represented in figure 27. The $\mathrm{Ca}_{3} \mathrm{C}_{4} \mathrm{O}_{9}$ baseline has a figure of merit of about 0.03 at $373 \mathrm{~K}$ and increases to 0.15 at $1073 \mathrm{~K}$. The doping of bismuth $\mathrm{x}=0.1$ not only increases the figure of from 0.03 to 0.08 at $373 \mathrm{~K}$, almost 3 times that of the baseline, but its final data point at $1073 \mathrm{~K}$ has kept this trend to best that of the $\mathrm{Ca}_{3} \mathrm{C}_{4} \mathrm{O}_{9}$ baseline by over two times its original value to achieve a ZT value of 0.33 at $1073 \mathrm{~K}$. 


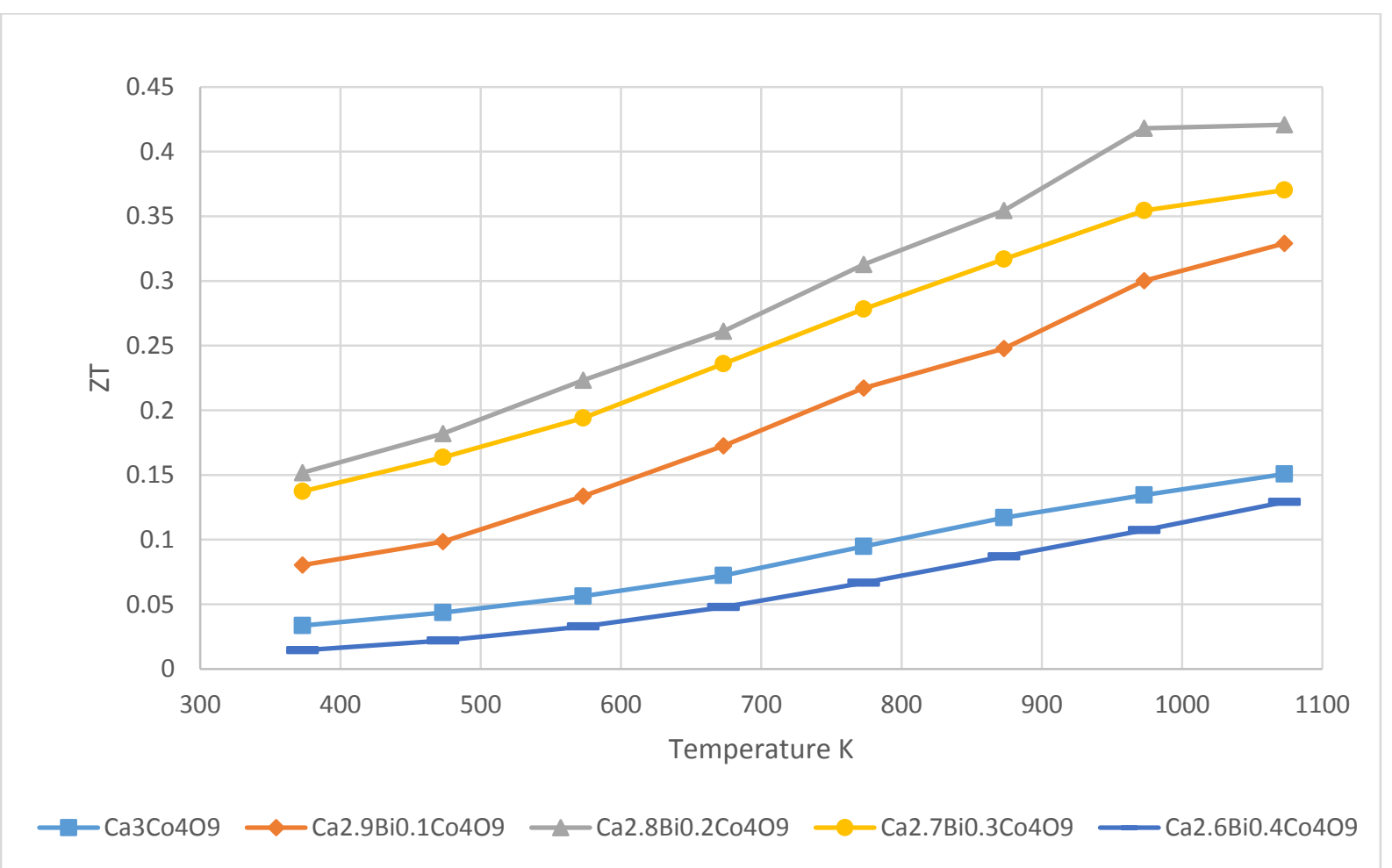

Figure 27 Temperature dependence of figure of merit for $\mathrm{Ca}_{3-x} \mathrm{Bi}_{x} \mathrm{Co}_{4} \mathrm{O}_{9}$ bismuth cation substitution.

Until recently, the best result reported on the cation substitution of bismuth by Yuheng Liu, et al. has a figure of merit of $\sim 0.25$ shown in figure 28. ${ }^{[7]}$ The data point of bismuth $x=0.1$ at $1073 \mathrm{~K}$ has already surpassed this with a value of $\sim 0.33$. This is great progress in the field of using oxides as a thermoelectric material. The trend continues with bismuth $x=0.2$ having a value of 0.15 at $373 \mathrm{~K}$ and a value of 0.42 at $973 \mathrm{~K}$ and $1073 \mathrm{~K}$. This value of 0.42 is almost three times the $\mathrm{Ca}_{3} \mathrm{Co}_{4} \mathrm{O}_{9}$ baseline at this specific temperature. Bismuth $\mathrm{x}=0.3$, although with a promising power factor, virtually overlapping that of bismuth $x=0.2$, has a lower figure of merit than bismuth $x=0.2$. This is a direct consequence of its large thermal conductivity as mentioned previously. This is the root cause for the reduction of its figure of merit. The over doped bismuth $\mathrm{x}=0.4$ not only has 
a low figure of merit but it actually drops below that of the pure baseline. This is caused by its large grain size and poor alignment resulting in a high electrical resistivity and low Absolute Seebeck coefficient in tandem with its high thermal conductivity.

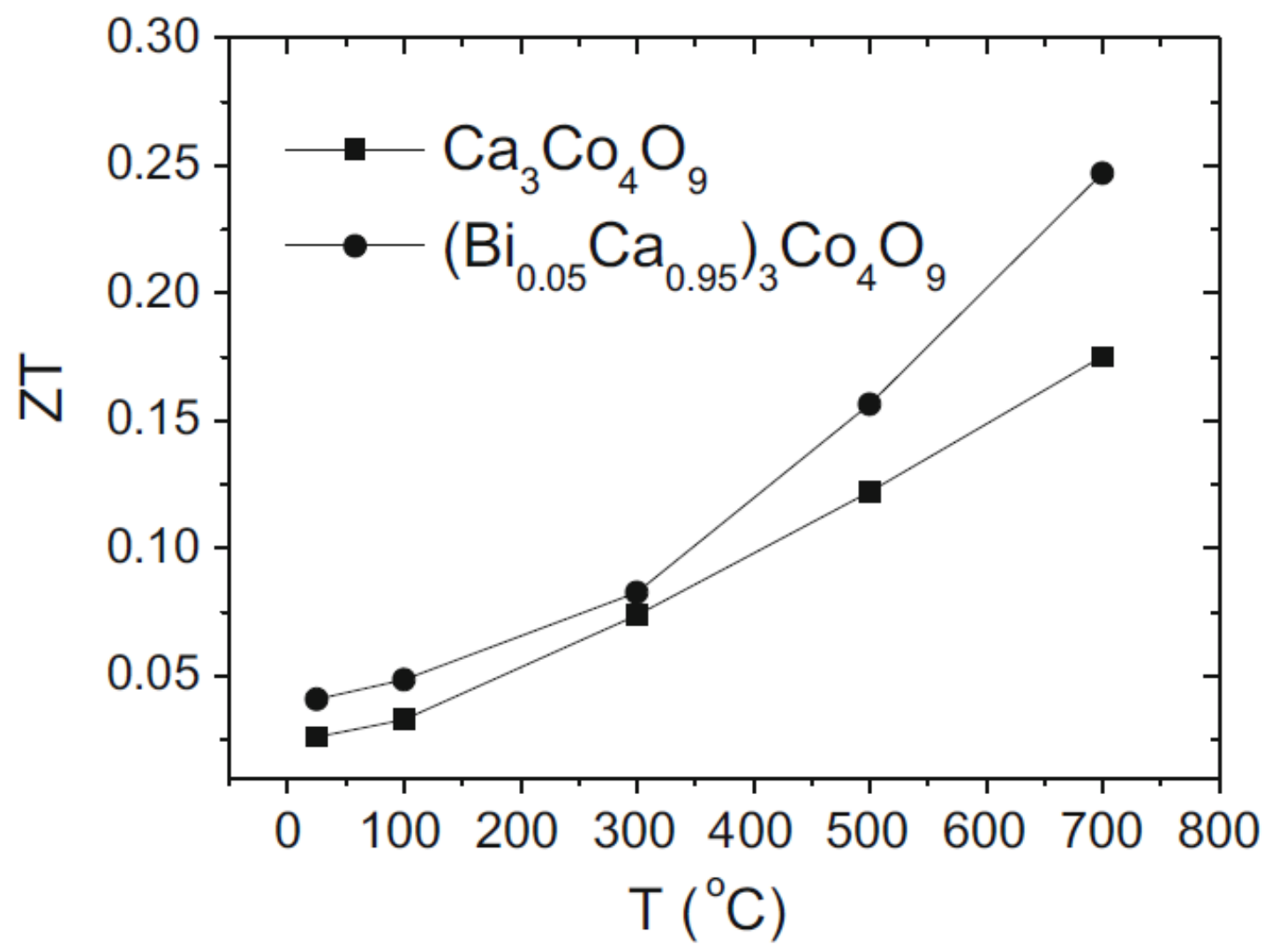

Figure 28 Temperature dependence of the $Z T$ values for undoped $\mathrm{Ca}_{3} \mathrm{Co}_{4} \mathrm{O}_{9}$ and $\left(\mathrm{Ca}_{0.95} \mathrm{Bi}_{0.05}\right)_{3} \mathrm{Co}_{4} \mathrm{O}_{9}\left[{ }^{[7]}\right.$

\subsection{Summary of results for Bismuth cation substitution in $\mathrm{Ca}_{3} \mathrm{Co}_{4} \mathrm{O}_{9}$}

Bismuth deposits itself at the grain interior to improve the crystal texture through increased grain growth along with grain alignment. It also deposited itself at the grain boundary causing segregation resulting in a carrier concentration reduction and 
enhancement of the Absolute Seebeck coefficient. The segregation at the grain boundary also helps to improve the crystal texture along with reducing the thermal conductivity. Low electrical resistivities of around $23 \mu \Omega m$ were achieved through doping the $\mathrm{Ca}_{3} \mathrm{C}_{4} \mathrm{O}_{9}$ with bismuth. The optimal concentration was found to be bismuth $x=0.2$ mainly attributing its high figure of merit due to its high power factor of $\sim 0.77 \mathrm{~mW} / \mathrm{mK}^{2}$ and its low thermal conductivity of $\sim 1.88 \mathrm{~W} / \mathrm{mK}$, both at $973 \mathrm{~K}$. Bismuth $\mathrm{x}=0.3$ still demonstrated a high power factor but its figure of merit suffers due to its slight increase in thermal conductivity caused by its increase in density. Bismuth $\mathrm{x}=0.4$, the over doped sample, has both poor electrical and thermal properties. Leading to a figure of merit lower than the pristine $\mathrm{Ca}_{3} \mathrm{Co}_{4} \mathrm{O}_{9}$. Figure 29 shows a drawing to demonstrate where the bismuth deposits and how it effects the grain alignment. The light red shows the presence of bismuth in the grains and the dark red line indicates the bismuth enriched grain boundaries.

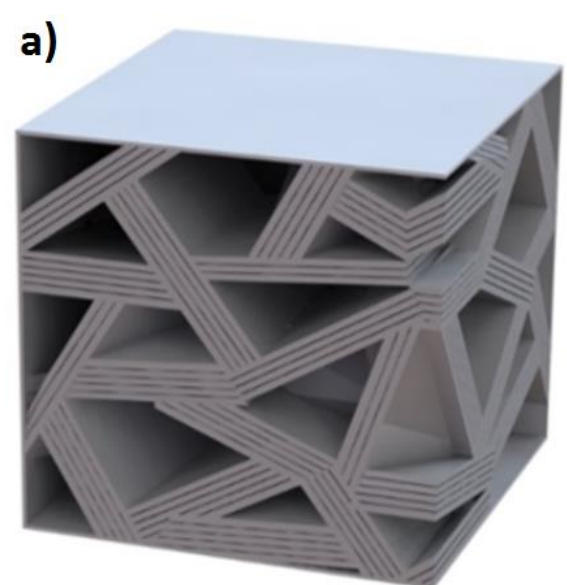

$\mathrm{Ca}_{3} \mathrm{Co}_{4} \mathrm{O}_{9}$ b)

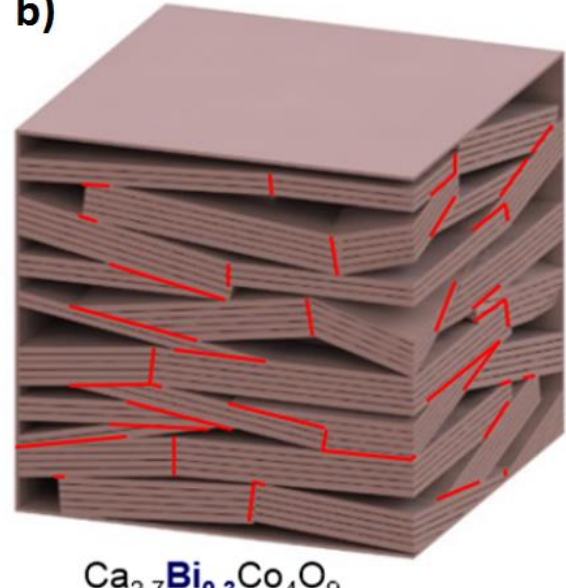

$\mathrm{Ca}_{2.7} \mathrm{Bi}_{0.3} \mathrm{Co}_{4} \mathrm{O}_{9}$
Bismuth in grain interior

Bismuth at grain boundary

Figure 29 Microstructure drawing of undoped $\mathrm{Ca}_{3} \mathrm{Co}_{4} \mathrm{O}_{9}$ and $\mathrm{Ca}_{2.7} \mathrm{Bi}_{0.3} \mathrm{CO}_{4} \mathrm{O}_{9}$. 
Analyzing bismuth's role at the grain boundary motivates the possibility to maximize the amount of segregation at the boundary. By using a non-stoichiometric addition method, which will be explained in detail in the following chapter, an attempt to force the bismuth solely to the grain boundary can be made in order to further enhance the absolute Seebeck coefficient. 


\subsection{References}

1 "Ionic substitution." A Dictionary of Earth Sciences. Encyclopedia.com. 28 Jul. 2017

2 Live Science Staff. Facts about Bismuth. September 5, 2013.

3 Gagnon, Steve. It's Elemental: The Periodic Table of Elements. The Element Bismuth. Jefferson Lab. 2017

4 Boundless. "Diamagnetism and Paramagnetism." Boundless Chemistry Boundless, Invalid Date Invalid Date. Invalid Date. Retrieved 28 Jul. 2017

5 Haoshan Hao, Qinglin He, Changqing Chen, Hongwei Sun and Xing Hu. Textured structure and anisotropic thermoelectric properties of $\mathrm{Ca}_{2.7} \mathrm{Bi}_{0.3} \mathrm{C}_{0} \mathrm{O}_{9}$ oxide prepared by conventional solid-state reaction. International Journal of Modern Physics B. Vol.23, No.1 pp.87-95, 2009.

6 Michitaka Ohtaki. Recent aspects of oxide thermoelectric materials for power generation from mid-to-high temperature heat source. Journal of the Ceramic Society of Japan 119 [11] 2011.

7 Yuheng Liu, Yuanhua Lin, Lei Jiang, Ce-Wen Nan, Zhijian Shen. Thermoelectric properties of $\mathrm{Bi}^{3+}$ substituted Co-based misfit-layered oxides. Journal of Electroceramics. Vol. 21, pp. 748-751, 2008.

8 Haoshan Hao, Limin Zhao and Xing Hu. Microstructure and Thermoelectric Properties of $\mathrm{Bi}$ - and $\mathrm{Cu}$-Substituted $\mathrm{Ca}_{3} \mathrm{CO}_{4} \mathrm{O}_{9}$ Oxides. Journal of Materials Science and Technology. Vol.25. No.1, 2009.

9 Siwen Li, Ryoji Funahashi, Ichiro Matsubara, Kazuo Ueno, Satoshi Sodeoka, and Hiroyuki Yamada. Synthesis and Thermoelectric Properties of the New Oxide Materials

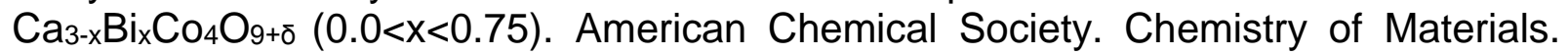
Vol.12, pp.2424-2427, 2000.

${ }^{10}$ David Moser, Lassi Karvonen, Sascha Populoh, Matthias Trottmann, Anke Weidenkarr. Influence of the oxygen content on thermoelectric properties of $\mathrm{Ca}_{3-\times} \mathrm{Bi}_{x} \mathrm{CO}_{4} \mathrm{O}_{9+\delta}$ system. Solid State Sciences. Vol. 13, Iss. 12, pp. 2160-2164, December 2011.

11 Masashi Mikami, Kanji Chong, Yuzuru Miyazaki, Tsuyoshi Kajitani, Takahiro Inoue, Satoshi Sodeoka and Ryoji Funahashi. Bi-Substitution Effects on Crystal Structure and Thermoelectric Properties of $\mathrm{Ca}_{3} \mathrm{C}_{4} \mathrm{O}_{9}$ Single Crystals. Japanese Journal of Applied Physics. Vol. 45, No. 5A, pp. 4131-4136, 2006.

12 Boyle, Cullen; Calvillo, Paulo; Chen, Yun; Barbero, Ever J.; Mclntyre, Dustin. Grain boundary segregation and thermoelectric performance enhancement of bismuth doped calcium cobaltite. Journal of the European Ceramic Society 36(3):601-607 Elsevier 2016 0955-2219

13 Ruomin Tian, Tianshu Zhang, Dewei Chu, Richard Donelson, Li Tao, Sean Li. Enhancement of high temperature thermoelectric performance in $\mathrm{Bi}, \mathrm{Fe}$ co-doped layered oxide-based material $\mathrm{Ca}_{3} \mathrm{C}_{4} \mathrm{O}_{9+\delta}$. Journal of Alloys and Compounds. 615:311315. July 4, 2014. 
${ }^{14}$ Murat Gunes, Macit Ozenbas. Journal of Alloys and Compounds. Vol.626, pp.360-367, 2015.

15 T. Sun, H. H. Hng, Q. Y. Yan, and J. Ma. Enhanced high temperature thermoelectric properties of $\mathrm{Bi}$-doped $\mathrm{c}$-axis oriented $\mathrm{Ca}_{3} \mathrm{C}_{4} \mathrm{O}_{9}$ thin films by pulsed laser deposition. AIP Journal of Applied Physics. 108, 083709. 2010.

${ }^{16}$ Zunyi Tian, Xiaohong, Wang, Jun Liu, Zhongjin Lin, Yangsen Hu, Yigui Wu, Chao Han, Zhiyu $\mathrm{Hu}$. Power factor enhancement induced by $\mathrm{Bi}$ and $\mathrm{Mn}$ co-substitution in $\mathrm{Na}_{\times} \mathrm{CoO}_{2}$ thermoelectric materials. Journal of Alloys and Compounds. 661, pp. 161-167, 2016.

${ }_{17}^{17}$ Ankam Bhaskar, Z.-R. Lin, Chia-Jyi Liu. Thermoelectric properties of Ca2.95Bio.05C04$\times \mathrm{Fe}_{\times} \mathrm{O}_{9+\delta}(0<\mathrm{x}<0.15)$. Energy Conversion and Management Vol.76, pp. 63-67, 2013.

${ }^{18}$ K. Rubešová, T. Hlásek, V. Jakeš, Š. Huber, J. Hejtmánek, D. Sedmidubský. Effect of a powder compaction process on the thermoelectric properties of $\mathrm{Bi}_{2} \mathrm{Sr}_{2} \mathrm{Co}_{1.8} \mathrm{O}_{x}$ ceramics. Journal of the European Ceramic Society, Vol.35, pp.525-531, 2015.

${ }^{19}$ Sajid Butt, Yaoyu Ren, Muhammad Umer Farooq, Bin Zhan, Rizwan Ur Rahman Sagar, Yuanhua Lin, Ce-Wen Nan. Enhanced thermoelectric performance of heavy-metals (M: $\mathrm{Ba}, \mathrm{Pb})$ doped misfit-layered ceramics: $\left(\mathrm{Ca}_{2}-\mathrm{M}_{\mathrm{x}} \mathrm{CoO}_{3}\right)_{0.62}\left(\mathrm{CoO}_{2}\right)$. Energy Conversion and Management. Vol.83, pp.35-41, 2014.

20 Susumu Fujii and Masato Yoshiya. Manipulating thermal conductivity by interfacial modification of misfit-layered cobaltites $\mathrm{Ca}_{3} \mathrm{CO}_{4} \mathrm{O}_{9}$. Journal of Electronic Materials, Vol.45, No.3, pp.1217-1226. 2016.

21 J. C. Diez, E. Guilmeau, M. A. Madre, S. Marinel, S. Lemonnier, A. Sotelo. Improvement of $\mathrm{Bi}_{2} \mathrm{Sr}_{2} \mathrm{Co}_{1.8} \mathrm{O}_{x}$ thermoelectric properties by laser floating zone texturing. Solid State Ionics, Vol.180, pp.827-830, 2009.

22 J. J. Shen, X. X. Liu, T. J. Zhu, X. B. Zhao. Improved thermoelectric properties of Ladoped $\mathrm{Bi}_{2} \mathrm{Sr}_{2} \mathrm{Co}_{2} \mathrm{O}_{9}$ - layered misfit oxides. Journal of Materials Science. Vol.44, pp.1889-1893, 2009.

${ }^{23}$ Kenji Tanabe, Ryuji Okazaki, Hiroki Taniguchi, and Ichiro Terasaki. Optical conductivity of layered calcium cobaltate $\mathrm{Ca}_{3} \mathrm{C}_{4} \mathrm{O}_{9}$. Journal of Physics: Condensed Matter. Vol.28, 2016.

${ }^{24}$ W. Koshibae, K. Tsutsui, and S. Maekawa. Thermopower in cobalt oxides. Physical Review B. Vol. 62, Num. 11, pp. 6869-6872. September 2000. 


\section{Chapter 5:}

\section{The Versatility of Bismuth doping through Non- stoichiometric Addition of $\mathrm{Ca}_{3} \mathrm{CO}_{4} \mathrm{O}_{9}$}

\subsection{Background and motivation for non-stoichiometric addition method}

Until recently, the most common and possibly the only doping method reported on has been that of cation substitution. Simply replacing specific ions of a baseline chemistry with a series of dopants which would cause different kinds of defects, within the material, leading to improved thermoelectric results. It was discovered, however, that in the event of doping a chemistry set there are some advantages to not replacing any of the ions from the baseline, rather adding to it and leaving the original chemistry untouched. Instead of dictating where the dopant will go by forcing it into vacancies created in lattice, through cation substitution, it allows the dopant to deposit itself where it so sees fit. Chapter four uses bismuth as a dopant utilizing the substitution method. Bismuth depositing itself at the grain interior and grain boundary allow it to be a great candidate for non-stoichiometric addition.

Non-stoichiometric addition is the addition of a dopant into the $\mathrm{Ca}_{3} \mathrm{C}_{4} \mathrm{O}_{9}$ without subtracting any existing ions such as the calcium ions substituted in chapter 4 . Instead of subtracting the calcium, the $\mathrm{Ca}_{3} \mathrm{C}_{4} \mathrm{O}_{9}$ chemistry will be untouched and just the specified concentration of the bismuth will be added. This allows bismuth ions the freedom to deposit themselves where they most naturally see fit which is where the energy is highest. Despite being close in ionic size with calcium there will be no reduction of ions thus 
presenting a greater amount of bismuth at the grain boundary in order to further enhance the room temperature Seebeck coefficient while still having the opportunity to fill in any calcium vacancies that may have occurred. By not preserving the original stoichiometry the possibility for secondary phases to occur increases and was investigated as well.

\subsection{Experimental Procedure}

$\mathrm{Ca}_{3} \mathrm{Bi}_{4} \mathrm{Co}_{4} \mathrm{O}_{9}$ powders where $\mathrm{u}=0.0,0.01,0.05,0.1,0.2,0.25,0.3,0.35,0.4$ were obtained through a sol-gel route in which non-stoichiometric ratios of $\mathrm{Ca}\left(\mathrm{NO}_{3}\right)_{2} \cdot 4 \mathrm{H}_{2} \mathrm{O}$, $\mathrm{Co}\left(\mathrm{NO}_{3}\right)_{2} \cdot 6 \mathrm{H}_{2} \mathrm{O}$ and $\mathrm{Bi}\left(\mathrm{NO}_{3}\right)_{2} \cdot 5 \mathrm{H}_{2} \mathrm{O}$ were mixed in deionized water. Ethylene glycol and polyethylene glycol were used to aid polymerization of the solution and to aid in particle size. ${ }^{[1]}$ Nitric acid was added to induce nitrate salts decomposition and facilitate new compound formation. ${ }^{[2]}$ The liquid solution was then submerged in a silicone oil bath on a hot plate and mechanically stirred at $353 \mathrm{~K}$ for 3 hours to achieve the sol-gel state. The sol-gel was then distributed to ceramic crucibles loosely covered with aluminum foil and put into a box furnace with a ramp rate of $10 \mathrm{~K}$ per minute and held at $773 \mathrm{~K}$ for 2 hours then cooled back to room temperature at a ramp rate of $10 \mathrm{~K}$ per minute. Holding the temperature here, at $773 \mathrm{~K}$, for the allotted time will ensure that any inorganic material is removed from the material. The ashes were then hand milled for 20 minutes via pestle and mortar. The powders were deposited in a tubular furnace where they were calcined in an oxygen rich induced environment with a ramp rate of $10 \mathrm{~K}$ per minute and held at $973 \mathrm{~K}$ for 4 hours, to achieve the $\mathrm{Ca}_{3} \mathrm{C}_{4} \mathrm{O}_{9}$ crystal lattice. The powders were then cooled down to room temperature at a rate of $10 \mathrm{~K}$ per minute. 
Two pellets were pressed, one to test its electrical properties and the other for its thermal properties. The calcined powders were pressed uniaxially with $1 \mathrm{GPa}$ of force and held for 40 minutes at $423 \mathrm{~K}$. The pellets' densities were obtained before they were put in to a tubular furnace to be sintered. The sintering process removes any extra moisture the pellet may contain and assures proper grain alignment. Pellets were sintered in an oxygen rich induced environment at $1233 \mathrm{~K}$ for 9 hours with a ramp rate of $10 \mathrm{~K}$ per minute. It was then cooled back to room temperature at a rate of $4 \mathrm{~K}$ per minute and once again the densities were taken. Pellets were cut into a $2 \mathrm{~mm} \times 3.5 \mathrm{~mm} \times 9 \mathrm{~mm}$ rectangular shapes and inserted into a Linseis LSR 3-Seebeck machine, subjected to a low pressure helium, He, environment where the pellet's electrical resistivity and Absolute Seebeck Coefficient were simultaneously determined by means of a dc four-probe method. Since helium is an inert gas it will not react or interfere with the measurement. A second pellet was cut along a different axis and inserted into the Linseis LFA 1000 machine to test its thermal conductivity. The pellet was cut to be $2 \mathrm{~mm}$ thick and polished to a diameter of $11-13 \mathrm{~mm}$. Any less than $11 \mathrm{~mm}$ and there would be room for heat to escape the holder, any more than $13 \mathrm{~mm}$ and the pellet would be too large for the holder. The thermal conductivity is calculated by the equation

$$
K=\lambda C_{p} \rho m
$$

Where $\lambda$ is the thermal diffusity in meters squared per second $\left(\mathrm{m}^{2} / \mathrm{s}\right), \mathrm{C}_{\mathrm{p}}$ is the specific heat capacity in joules per kilogram kelvin $(\mathrm{J} / \mathrm{kg} \cdot \mathrm{K})$, and $\rho_{\mathrm{m}}$ is the mass density in grams per centimeter cubed $\left(\mathrm{g} / \mathrm{cm}^{3}\right)$. As stated in the first section, these are the three values needed in order to obtain the materials' ZT value. For bismuth addition, nine different chemistries were used. The first chemistry is the baseline of $\mathrm{Ca}_{3} \mathrm{C}_{4} \mathrm{O}_{9}$ and the other eight 
are dopings of bismuth; $\mathrm{Ca}_{3} \mathrm{Bi}_{4} \mathrm{Co}_{4} \mathrm{O}_{9}$ where $\mathrm{u}=0.01,0.05,0.1,0.2,0.250 .3,0.35$ and 0.4 .

\subsection{Simultaneously doping the lattice and grain boundary}

5.3.1 Increasing the carrier mobility through bismuth non-stoichiometric addition

Since bismuth 0.01 is such a small amount to dope with, it can be seen in figure 30 that the change in resistivity between the baseline and bismuth 0.01 is very trivial. However, at bismuth 0.05 a jump in the trend can be seen. At room temperature, the electrical resistivity of the bismuth 0.05 concentration is around $43 \mu \Omega \mathrm{m}$. Just below this, following suit with the trend lies bismuth 0.1 at $39 \mu \Omega \mathrm{m}$. Unlike the baseline and bismuth 0.01 's electrical resistivity which increased from $70 \mu \Omega m$ to $100 \mu \Omega m$ a $30 \mu \Omega m$ jump, bismuth 0.05 and bismuth 0.1 's resistivity only increases from around $40 \mu \Omega m$ at room temperature to $60 \mu \Omega \mathrm{m}$ at $1073 \mathrm{~K}$ which is only a $20 \mu \Omega \mathrm{m}$ jump. The trend continues up to bismuth 0.3 with each increased concentration having a lower resistivity than the last. The room temperature electrical resistivities of bismuth $0.2,0.25$, and 0.3 are all between 17 and $21 \mu \Omega m$ and increase to between 40 and $49 \mu \Omega m$ at $1073 \mathrm{~K}$. Although each concentration increased in electrical resistivity from room temperature to $1073 \mathrm{~K}$, it is noticeable that with each increased amount of bismuth the resistivity starts off lower and increases less. This trend insinuates that the addition of bismuth improves the grain texture and size and aids in the alignment of the grains just as it has with the set utilizing the cation substitution method. ${ }^{[3]}$ Samples using the non-stoichiometric addition method actually have a lower electrical resistivity than that of the cation substitution samples at its optimum concentrations. The 0.35 and 0.4 content samples experience a significant 
increase in their electrical resistivities most likely demonstrating an over doping of the samples. Furthermore it is notable that 0.35 has a higher electrical resistivity than the 0.4 concentration. Although this can indirectly insinuate that another drop can be expected if the concentration were to be increased, due to the electrical resistivity trends followed by these samples it is evident that both are in fact over doped and this slight variation can be explained through slight process variation due to error. This will be further elucidated when their absolute Seebeck coefficients are discussed.

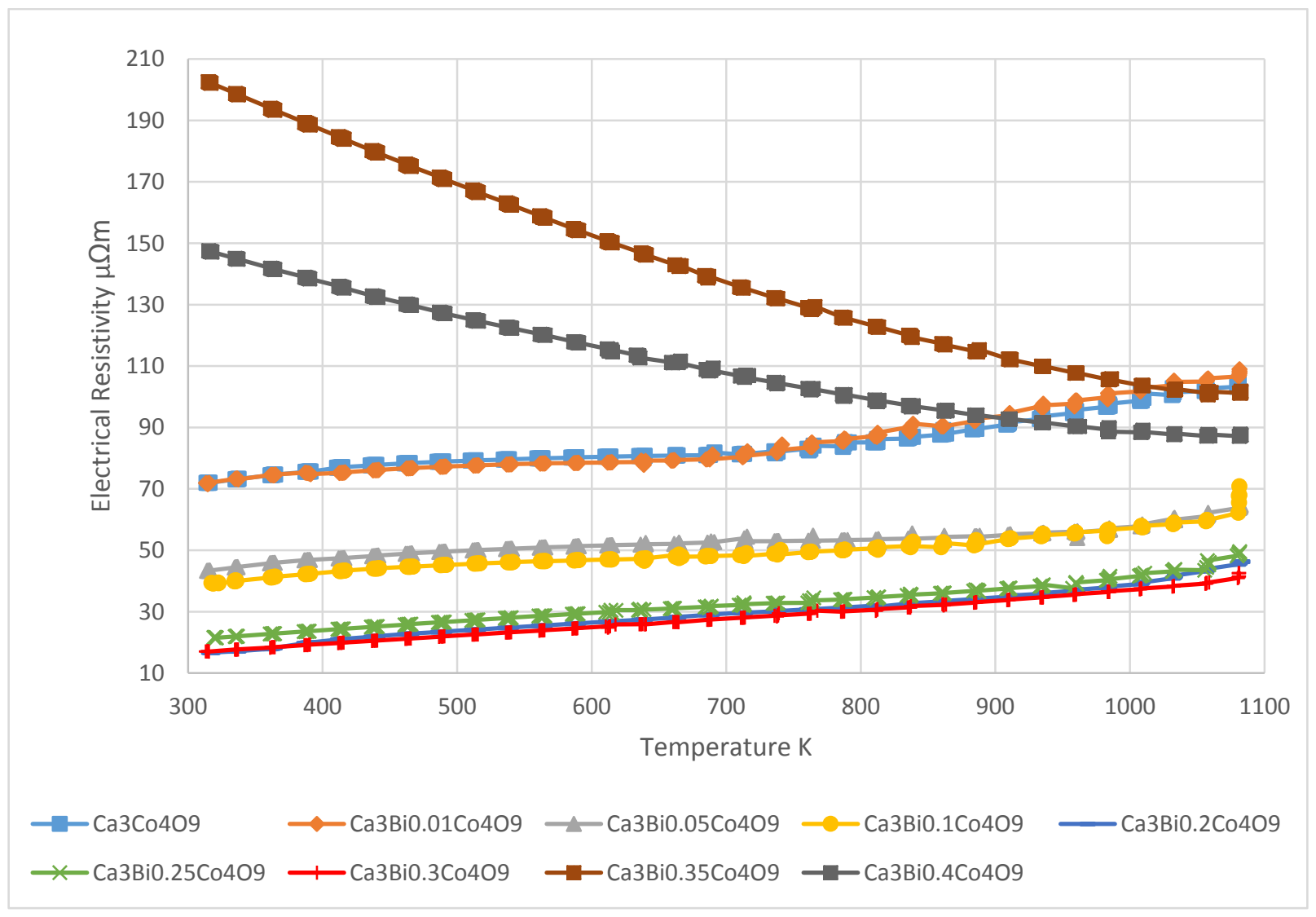

Figure 30 Temperature dependence of electrical resistivity for $\mathrm{Ca}_{3} \mathrm{Bi}_{4} \mathrm{Co}_{4} \mathrm{O}_{9}$ bismuth non-stoichiometric addition. 
5.3.2 Improvement of grain alignment along with boundary segregation

Figure 31 shows grain development the from cross-sectional SEM images of surface fractured pellets. Image (a) shows the undoped $\mathrm{Ca}_{3} \mathrm{Co}_{4} \mathrm{O}_{9}$ where (b), (c), (d), and (e) show the $0.1,0.2,0.3$ and 0.35 concentrations respectively. The improved grain alignment is evident as the content of bismuth increases. The optimal concentration, of 0.3 , shown in image $(d)$ is in stark comparison with that of the undoped $\mathrm{Ca}_{3} \mathrm{C}_{4} \mathrm{O}_{9}$ shown in image (a), with its preferred alignment along the a- and b-plane. Image (e) shows a glassy like crystal structure of the over doped $\mathrm{Ca}_{3} \mathrm{Bi}_{0.35} \mathrm{CO}_{4} \mathrm{O}_{9}$ sample with grains larger than $20 \mu \mathrm{m}$ and no preferred direction. This improved crystal texture in image (d) is caused by the doping of bismuth into the $\mathrm{Ca}_{3} \mathrm{C}_{4} \mathrm{O}_{9}$ lattice which improves its alignment as bismuth increases to its optimal concentration of 0.3 .
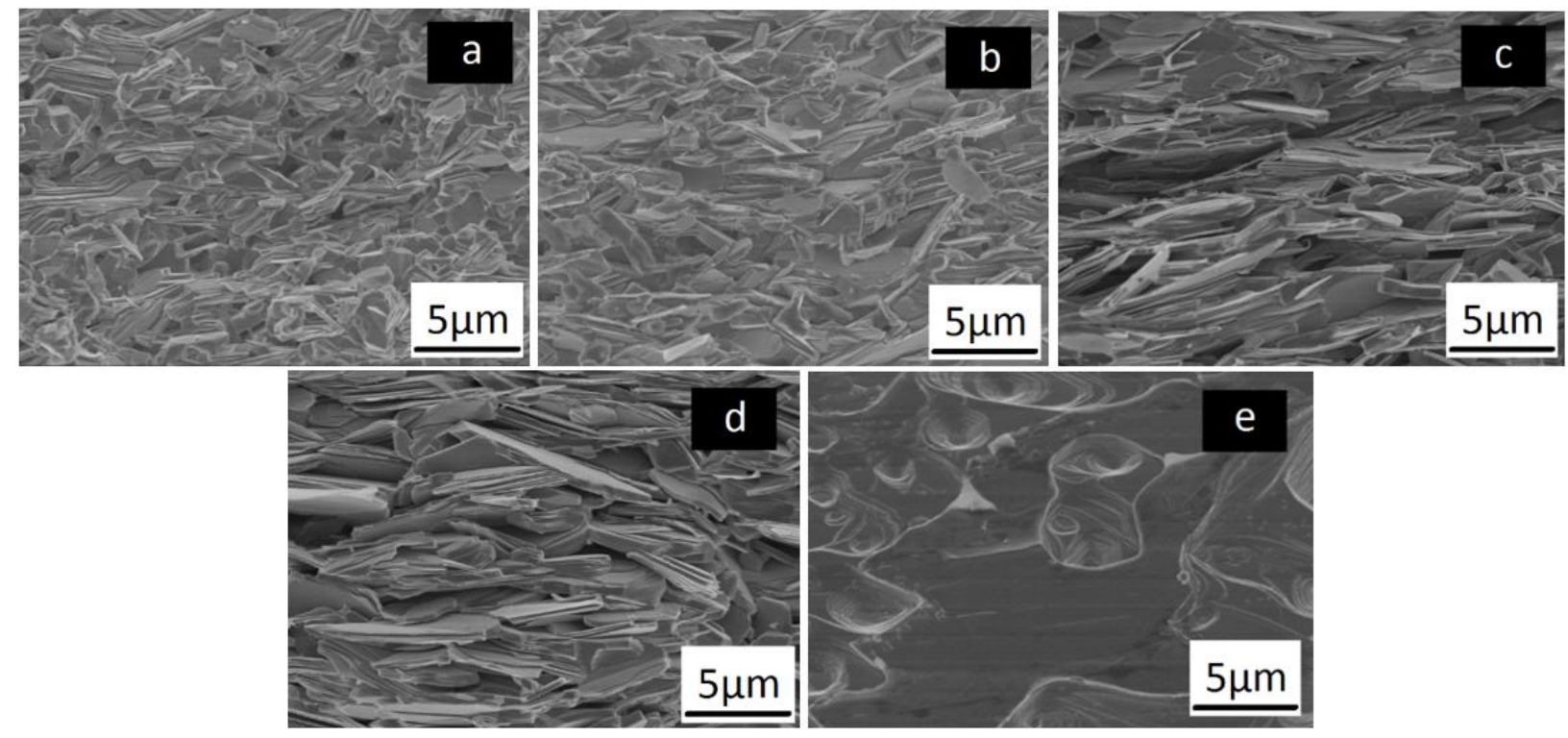

Figure 31 SEM cross-sectional view of undoped $\mathrm{Ca}_{3} \mathrm{CO}_{4} \mathrm{O}_{9}$ and doped $\mathrm{Ca}_{3} \mathrm{Bi}_{4} \mathrm{CO}_{4} \mathrm{O}_{9}$ with $u=0.0,0.1,0.2,0.3$, and 0.35 . 
Apparent in figure 32 is that once the bismuth is added to the baseline the Seebeck coefficient increases anywhere from $20-30 \mu \mathrm{V} / \mathrm{K}$ at room temperature reaching a range of $140-150 \mu \mathrm{V} / \mathrm{K}$ which is consistent with the findings of $\mathrm{K}$. Rubešová et al. ${ }^{[4]}$ This is, in fact, greater than that experienced through the cation substitution method using the same dopant. This increase is due to bismuth depositing itself and segregating at the grain boundary as well as depositing itself at the grain. The unique thing about the addition process is that the doping element is allowed to deposit itself where it sees fit, as opposed to deliberately forcing it go a specific location.

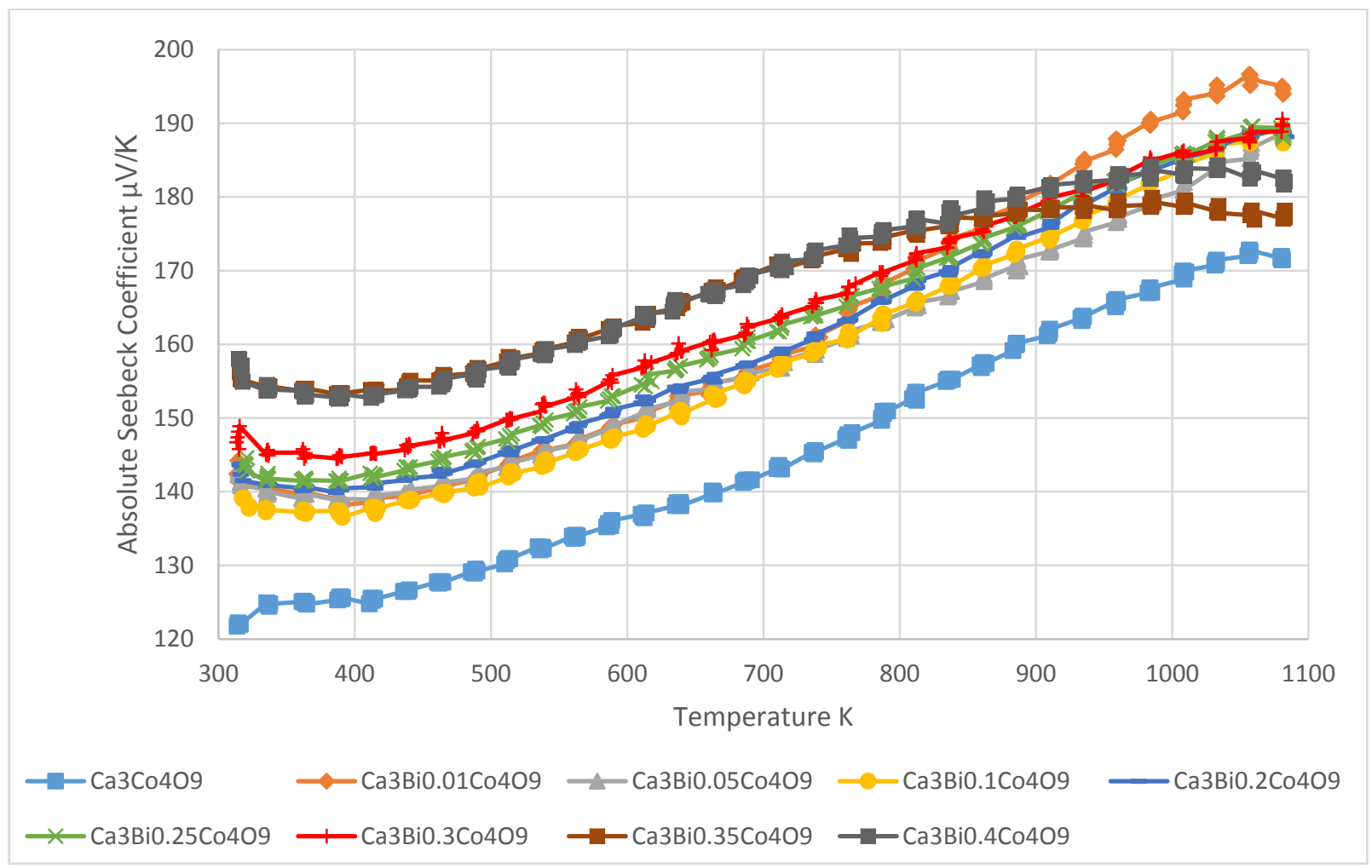

Figure 32 Temperature dependence of absolute seebeck coefficient for $\mathrm{Ca}_{3} \mathrm{Bi}_{4} \mathrm{Co}_{4} \mathrm{O}_{9}$ bismuth non-stoichiometric addition. 
Bismuth deposits itself at the grain, due to a similar ionic size to calcium, causing a significant reduction in the electrical resistivity. ${ }^{[3,5]}$ This can be shown via the PANalytical XPert-Pro X-Ray Diffraction which used Cu K-alpha radiation with $45 \mathrm{kV}$ tension and 40 $\mathrm{mA}$ of current at room temperature. Figure 33 is a graph of those results with figure 34 focusing in on the (004) peak clearly showing a shift in peak to a lower angle with increasing bismuth. This means that the d-spacing between crystal planes must be increasing, indicating a growth in lattice size. This is an indicator that the bismuth still deposits itself into the lattice upon doping, even in a scenario where calcium atoms are not being removed. It can also be seen that there is a presence of bismuth at the grain boundary causing the segregation which manifests itself as an increase in the Absolute Seebeck Coefficient. ${ }^{[3]}$

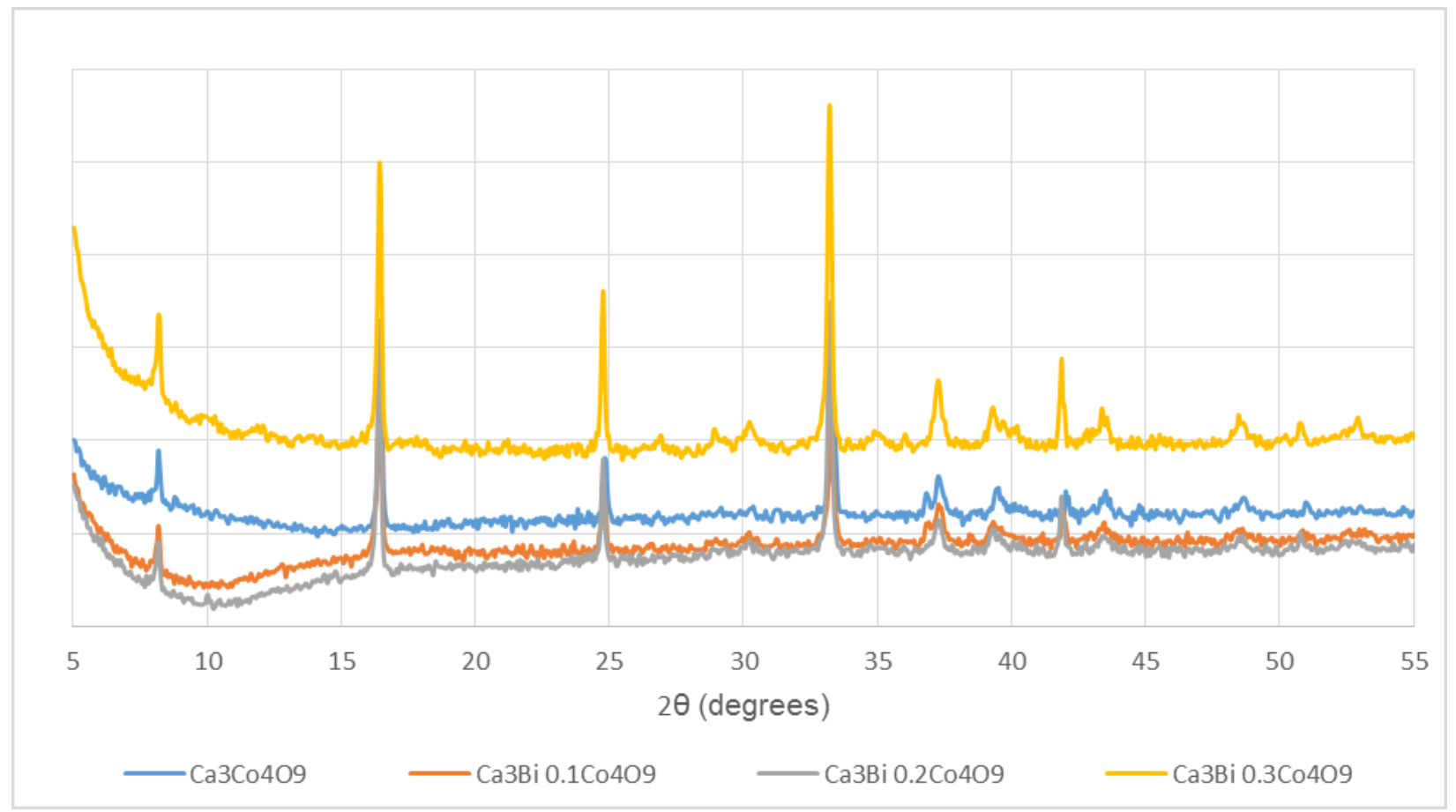

Figure 33 X-Ray Diffraction patterns of undoped and doped $\mathrm{Ca}_{3} \mathrm{Co}_{4} \mathrm{O}_{9}$. 


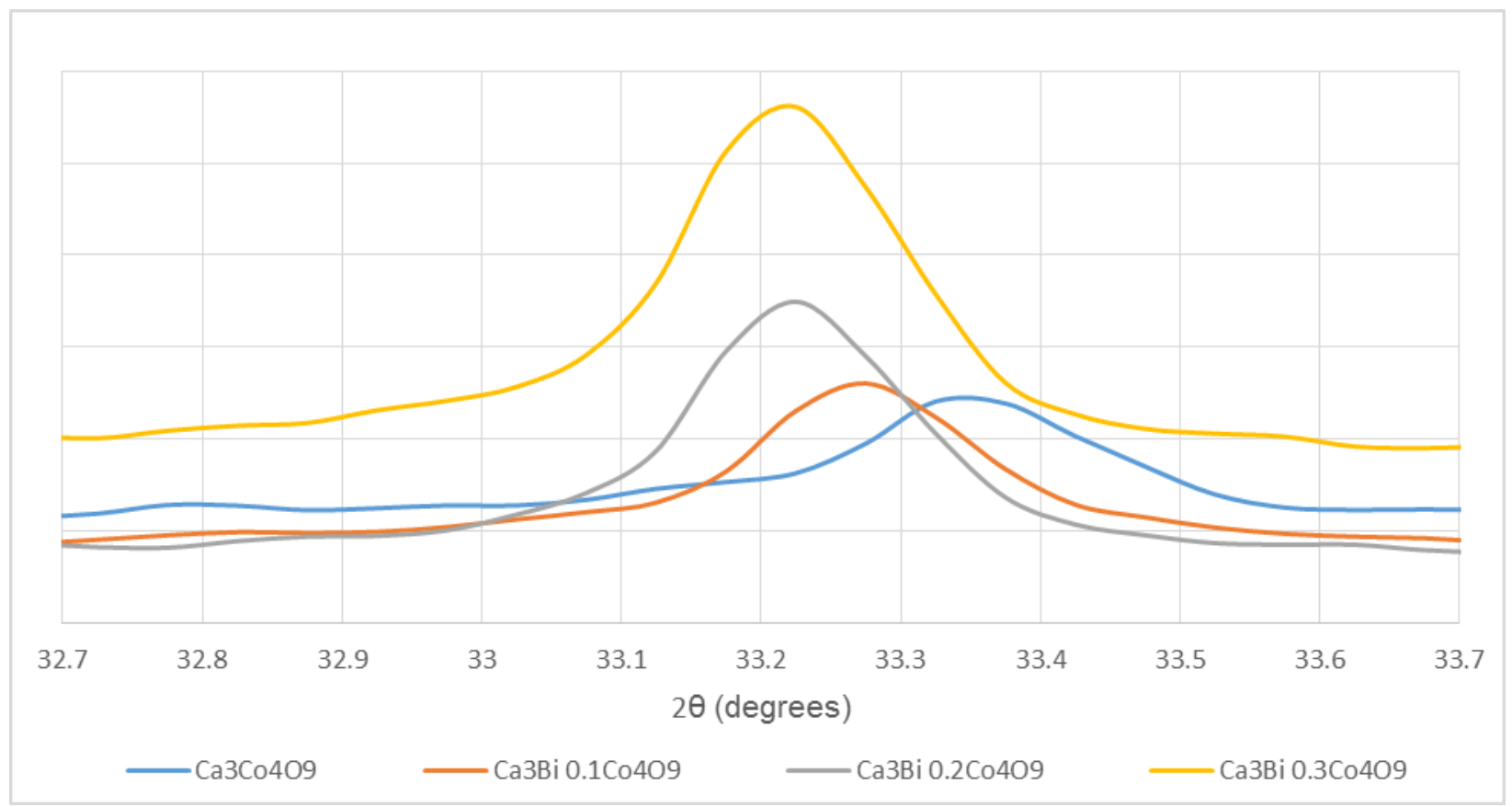

Figure 34 (004) peak from X-Ray Diffraction patterns of undoped and doped $\mathrm{Ca}_{3} \mathrm{Co}_{4} \mathrm{O}_{9}$ powders.

Bismuth acts as a filter at the grain boundary to reduce the carrier concentration thus enhancing the Seebeck Coefficient. ${ }^{[6]}$ However, a decrease in carrier concentration usually leads to an increase in electrical resistivity. ${ }^{[7,8]}$ Both electrical properties can be simultaneously improved with the help of its carrier mobility. ${ }^{[8]}$ During substitution the bismuth deposits itself at the calcium site increasing the carrier mobility. ${ }^{[2]}$ Since bismuth can deposit itself freely during addition some will still find its way to where there are any calcium deficiencies and deposit itself there into the grain once again increasing the carrier mobility, improving crystal texture and in turn reducing the electrical resistivity. [9] This is why there is an increase in Seebeck coefficient at the grain boundary without negatively affecting its electrical resistivity. ${ }^{[10]}$ 
However, there is an elegant balance to this method. Too large of a reduction to the carrier concentration will eventually have an overall deleterious effect since at a certain point this becomes too influential for the mobility to counterbalance. This is exactly why in the over doped samples the electrical resistivity has a drastic increase in electrical resistivity mirrored by a significant decrease in its Seebeck coefficient at high temperatures. ${ }^{[2]}$ The growth at the grain boundary dominates causing too large of a reduction in carrier concentration. Highly doped materials tend to have a smaller mobility and a smaller mean free time. ${ }^{[10,11]}$ The mean free time is the time it takes for successive impacts of particles and a smaller mean free time means more scattering and less mobility.

Taking a closer look at the low temperature regime in figure 35 can further elucidate the scenario and create a clearer mental image. Figure 35 (a) gives a closer look the electrical resistivity and 36 (b) gives a closer looks at the Absolute Seebeck Coefficients, both in the low temperature regime. It can be seen that there are three main groupings of the Absolute Seebeck Coefficients in figure 35 (b). The first is the baseline which has an expected low Seebeck coefficient. The second grouping contains all of the dopings, up to but not including the over doped samples, ranging from 139-149 $\mu \mathrm{V} / \mathrm{K}$. As stated earlier the increase in Seebeck coefficient is due to the introduction of bismuth to the grain boundary to decrease carrier concentration. Each successive increase in doping also increases the Seebeck coefficient within its respective grouping. The third grouping containing bismuth 0.35 and 0.4 dopings has the highest Seebeck coefficient in the low temperature regime which proves that even at these high concentrations the carrier concentration is still decreasing due to the bismuth at the grain boundary. Along with the 
decrease in concentration there is also a decrease in mobility therefore it is at these concentrations where the negative effects of the over doping become evident. By examining figure 35 (a) it can be seen that it is at these concentrations that the electrical resistivities make a massive leap due to the decrease in mobility and in turn will decrease its overall power factor to the point where its large Seebeck coefficient cannot compensate for this reduction in electrical resistivity.

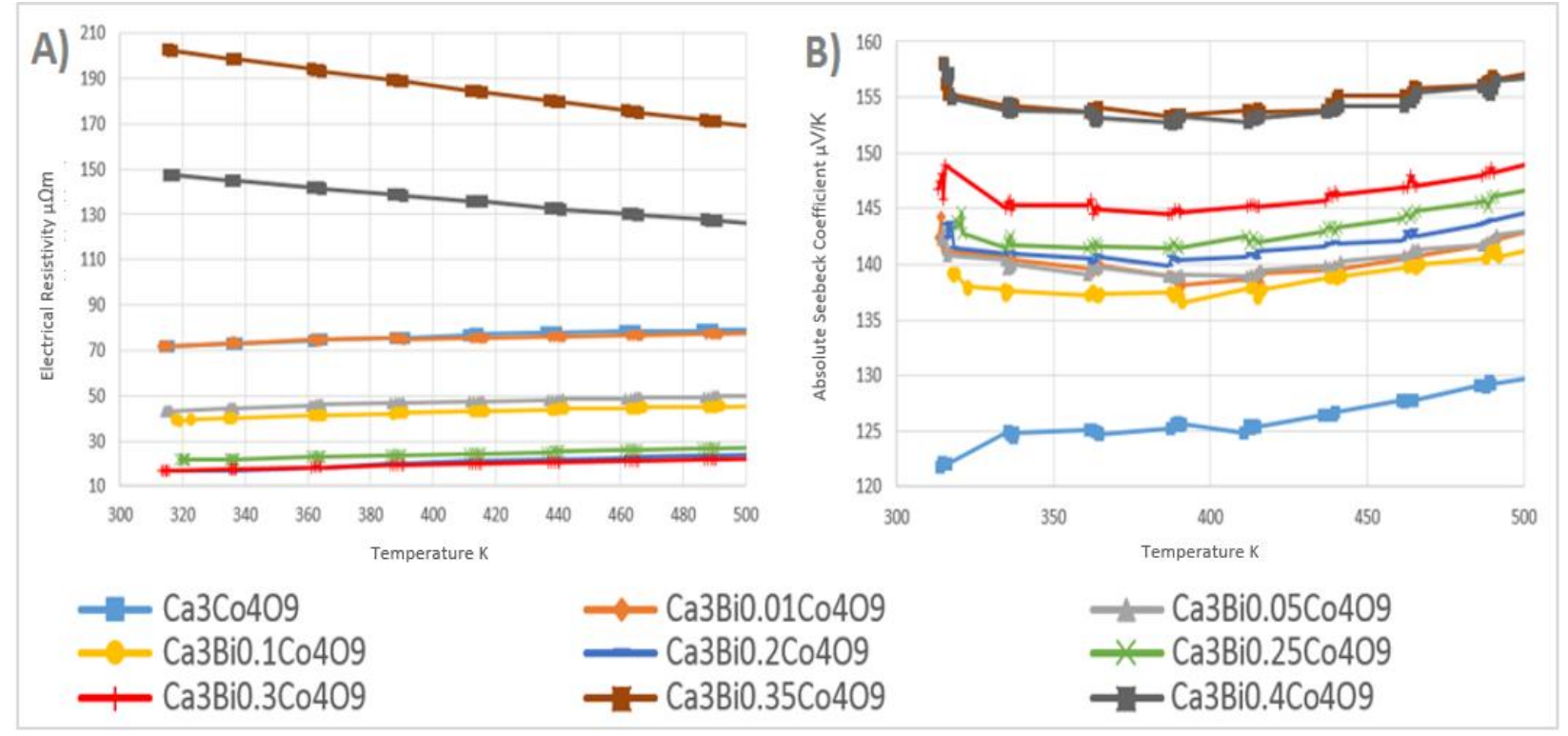

Figure 35 (a) Temperature dependence of electrical resistivity and (b) absolute seebeck coefficient of $\mathrm{Ca}_{3} \mathrm{Bi}_{4} \mathrm{CO}_{4} \mathrm{O}_{9}$ bismuth non-stoichiometric addition.

A low electrical resistivity of $17 \mu \Omega \mathrm{m}$ and a vastly enhanced Absolute Seebeck coefficient of $148 \mu \mathrm{V} / \mathrm{K}$ at $315 \mathrm{~K}$ give rise to a high power factor of $1.3 \mathrm{~mW} / \mathrm{mK}^{2}$ which is over seven times that of the $0.17 \mathrm{~mW} / \mathrm{mK}^{2}$ power factor from the undoped $\mathrm{Ca}_{3} \mathrm{Co}_{4} \mathrm{O}_{9}$, both shown in figure 36 . It also achieves a power factor of $\sim 0.94 \mathrm{~mW} / \mathrm{mK}^{2}$ at $983 \mathrm{~K}$ which is three time larger than the $0.35 \mathrm{~mW} / \mathrm{mK}^{2}$ power factor from the undoped $\mathrm{Ca}_{3} \mathrm{Co}_{4} \mathrm{O}_{9}$, allowing it to have one of the highest power factors across this temperature range. 


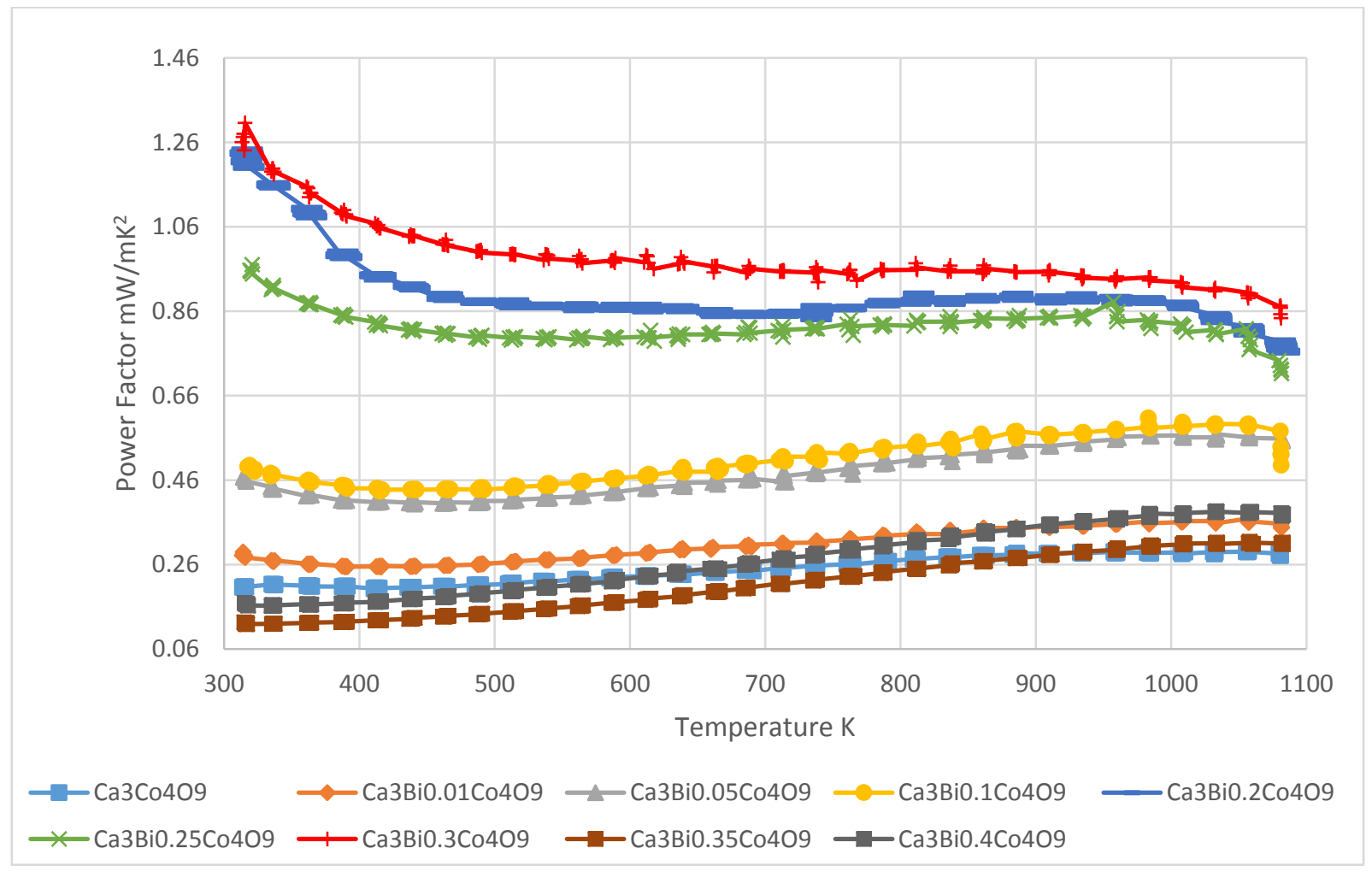

Figure 36 Temperature dependence of the power factor for $\mathrm{Ca}_{3} \mathrm{Bi}_{4} \mathrm{Co}_{4} \mathrm{O}_{9}$ bismuth nonstoichiometric addition.

5.3.3 Significance of Phonon Scattering and a small Mean Free Path (MFP)

The thermal conductivity is a less controllable parameter than the electrical properties, as stated earlier, due to the Wiedemann-Franz Law. This is reflected in the seemingly inconsistent jumps in thermal conductivity as the dopings are increased. The reason for this is the coupling of the electron thermal conductivity and the lattice or phonon thermal conductivity. The phonon thermal conductivity can be decreased by interface phonon scattering at the grain boundaries which results in a decrease in phonon mean free path. ${ }^{[1,10,12]}$ As mentioned bismuth allows the $\mathrm{Ca}_{3} \mathrm{C}_{4} \mathrm{O}_{9}$ to have a shorter mean free path. Also a smaller grain size means more grains and in turn more grain boundaries and a stronger phonon scattering at the boundaries. This is yet another reason why bismuth 
is a good candidate for thermoelectric materials. Bismuth allows for grain growth in order to enhance the electrical properties but does not grow to where it will negatively affect the thermal properties. Defects such as impurities, point defects, and dislocations all contribute to scattering at the boundaries as well. ${ }^{[1,12,13,14]}$ The power factor of bismuth 0.4 was poor due to the grain growth and decrease in mobility and its thermal properties would most likely be poor as well due to a higher density considering the density increases with increasing amounts of bismuth. For this concentration only the electrical properties were tested.

The doping of bismuth 0.01 was not enough to trigger the grain growth or have any kind of presence at the grain boundary. It also did not affect the sample's density to a large extent so no significant results came from its thermal conductivity, causing it to be very similar to the conductivity of that of the baseline. The doping of bismuth 0.05 is where its first drop in thermal conductivity can be seen in figure 37 , with bismuth 0.35 surprisingly having the lowest conductivity. A lower density allows for a higher rate of phonon scattering at the grain boundary which is responsible for its low thermal conductivity. Although it has such a low thermal conductivity, coupled with its poor power factor it would most likely not have a high figure of merit. 


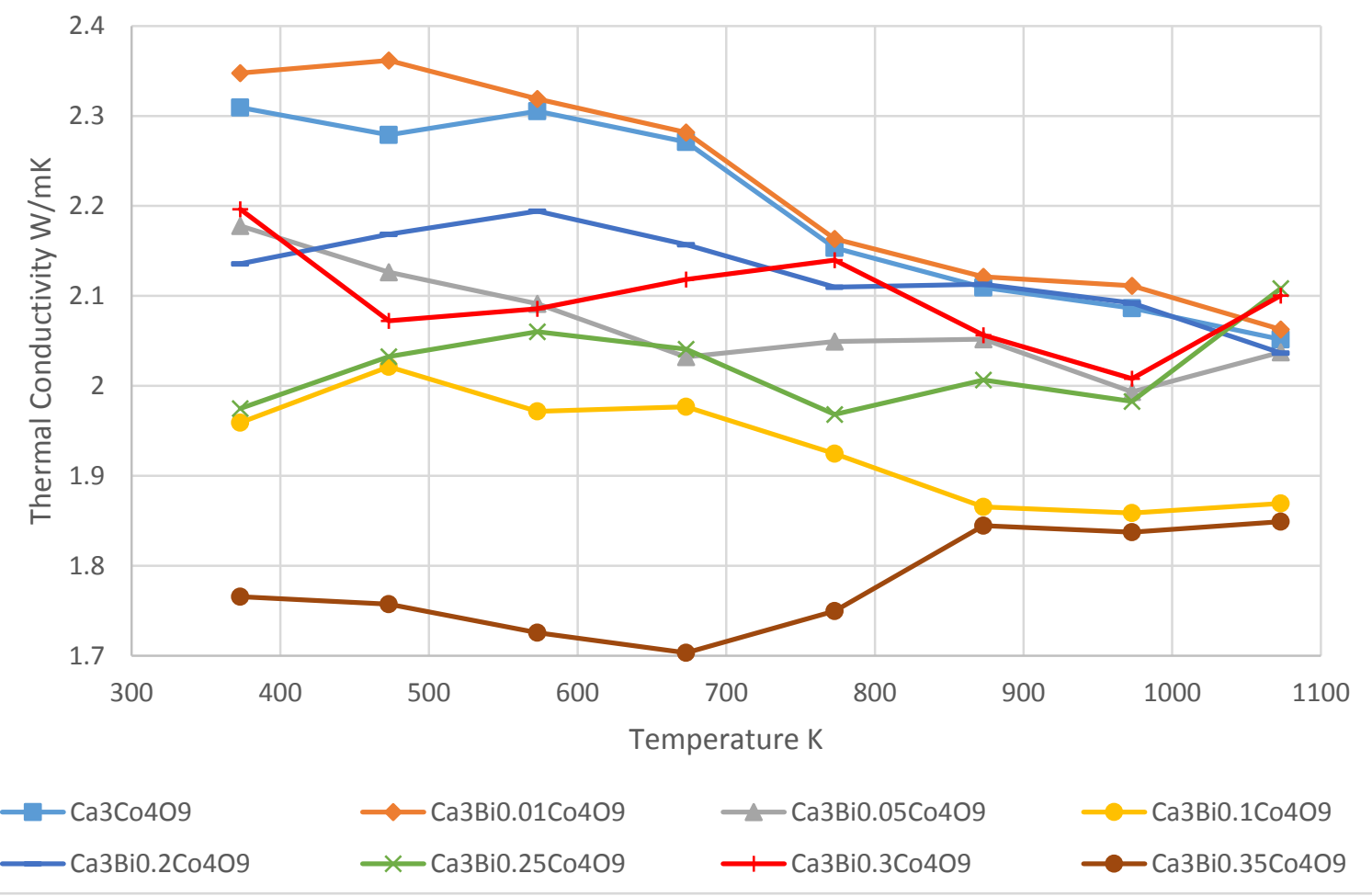

Figure 37 Temperature dependence of thermal conductivity for $\mathrm{Ca}_{3} \mathrm{Bi}_{4} \mathrm{Co}_{4} \mathrm{O}_{9}$ bismuth non-stoichiometric addition.

5.3.4 Figure of merit of bismuth non-stoichiometric addition doped $\mathrm{Ca}_{3} \mathrm{C}_{4} \mathrm{O}_{9}$

The power factor of the $\mathrm{Ca}_{3} \mathrm{Bi}_{4} \mathrm{Co} 4 \mathrm{O} 9$ samples increases with each sample of increased bismuth concentration. This is due to the simultaneous decrease in electrical resistivity caused by its grain growth, texture improvement, and grain alignment along with its increase in the Absolute Seebeck Coefficient caused by the grain boundary segregation. This trend continues up to and includes bismuth 0.3 which has the highest power factor of $\sim 0.94 \mathrm{mWm}^{-1} \mathrm{~K}^{-2}$ at $973 \mathrm{~K}$. This is more than 3 times as high as the baseline which is $\sim 0.29 \mathrm{mWm}^{-1} \mathrm{~K}^{-2}$ at $973 \mathrm{~K}$. The two over doped samples of bismuth 0.35 and 0.4 have a lower power factor than the baseline which is due to the drastic increase in their electrical resistivities as mentioned earlier. 
The figure of merits shown in figure 38 demonstrate that the bismuth 0.3 doped sample at the low temperature regime, specifically at $373 \mathrm{~K}$, starts at $\sim 0.19$ which is higher than the high temperature regime of the baseline and bismuth 0.01 at $1073 \mathrm{~K}$, which are 0.15 and 0.18 respectively. The bismuth 0.3 doped sample continues to increase with increasing temperature until it reaches a value of 0.45 at $973 \mathrm{~K}$ and $1073 \mathrm{~K}$. This is 3 times higher than the 0.15 value of the undoped sample at the same temperature.

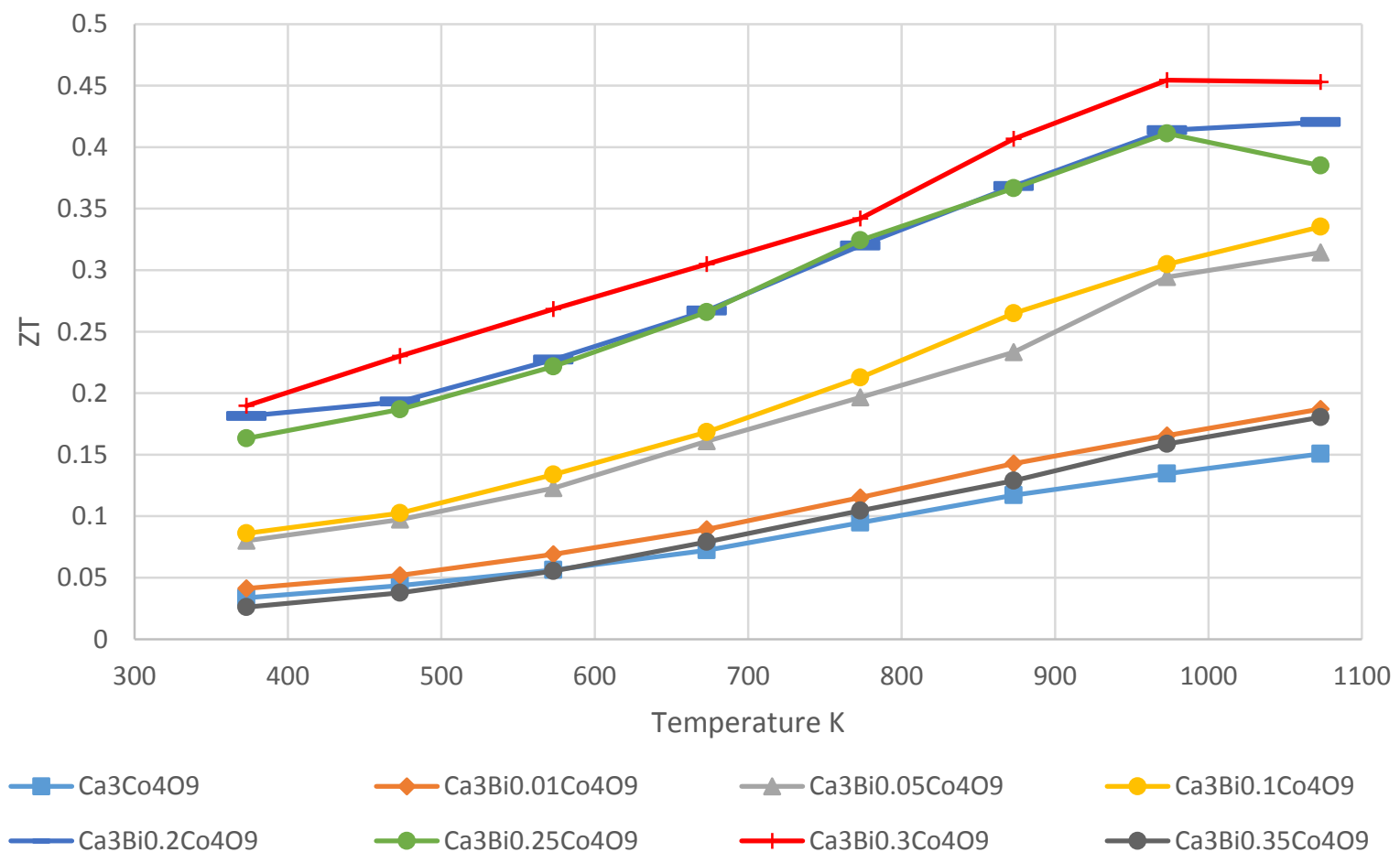

Figure 38 Temperature dependence of figure of merit for $\mathrm{Ca}_{3} \mathrm{Bi}_{4} \mathrm{Co}_{4} \mathrm{O}_{9}$ bismuth nonstoichiometric addition.

\subsection{Effects of processing parameters on electrical \& thermal properties}

The concentration with the best figure of merit was analyzed to see if its thermoelectric properties can be further enhanced. The pressing parameters of the 
bismuth 0.3 sample were varied as the fabrication process was held constant. The two main experimental groups for this exercise were comparing cold pressing to hot pressing (hot pressing was used for the set previously discussed in this chapter). During the cold pressing procedure, pellets were pressed at $298 \mathrm{~K}$ for 10 minutes. The hot pressing procedure was held at a temperature of $423 \mathrm{~K}$ for 40 minutes. This allows the ability of a denser pellet since the heat can cause the forming of a liquid phase enabling the grains to better align themselves. As mentioned, a higher density will usually enhance the electrical properties but the thermal properties almost always suffer since they are directly related to the density.

Within each pressing group, another parameter was varied in order to optimize their results. Under the cold pressing condition, pellets were pressed at $0.5 \mathrm{GPa}, 0.75$ $\mathrm{GPa}$ and $1 \mathrm{GPa}$ and under the hot pressing condition, pellets were pressed at $0.75 \mathrm{GPa}$ and $1 \mathrm{GPa}$. Different pressures will once again impact the pellets' density. Since the electrical and thermal properties are so intimately intertwined, mainly through their density's, there is hope to find the perfect balance of enhanced electrical properties without adversely affecting the thermal and vice versa. By varying these pressing parameters the hope is that the electrical and thermal properties will indeed find that balance where the greatest figure of merit can be achieved.

\subsubsection{Electrical performance of $\mathrm{Ca}_{3} \mathrm{Bi}_{3} \mathrm{C}_{4} \mathrm{O}_{9}$ varying ceramic processing parameters} Increased pressing pressure yields a denser material and enhances the electrical resistivity. The only sample that does not follow suit with this trend is the sample hot pressed at $0.75 \mathrm{GPa}$ which can be seen in figure 39 . It actually has a lower electrical resistivity at high temperatures than the pellet that was cold pressed at $1 \mathrm{GPa}$. This is 
due to the heat applied during the pressing process. The heat created a liquid phase of the bismuth in order to allow for better grain alignment giving it a higher density which lead to a lower electrical resistivity. The pellet hot pressed at $0.75 \mathrm{GPa}$ has a density of $4.25 \mathrm{~g} / \mathrm{cm}^{3}$ while the cold pressed pellet pressed at $1 \mathrm{GPa}$ has a density of $3.95 \mathrm{~g} / \mathrm{cm}^{3}$. This shows that the temperature of the pressing process effects the pellets density much greater than the pressure at which it is pressed.

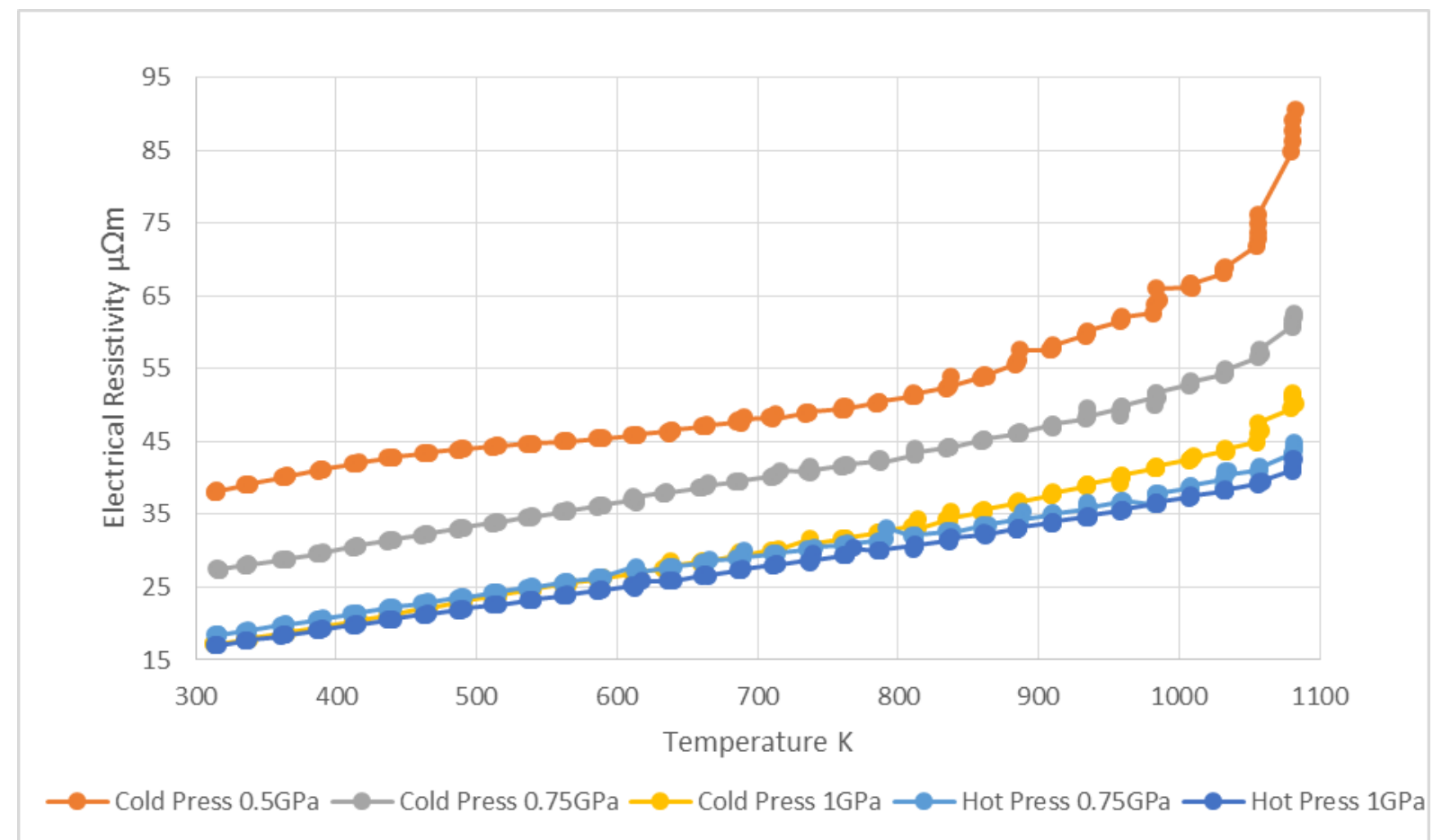

Figure 39 Temperature dependence of electrical resistivity for $\mathrm{Ca}_{3} \mathrm{Bi}_{0.3} \mathrm{CO}_{4} \mathrm{O}_{9}$ bismuth non-stoichiometric addition with pressing parameter variation.

The pellet pressed at $423 \mathrm{~K}$ with a pressure of $0.75 \mathrm{GPa}$ achieves an electrical resistivity of $\sim 17 \mu \Omega \mathrm{m}$ at $315 \mathrm{~K}$, same as the two pellets pressed at $1 \mathrm{GPa}$. They begin to differ at high temperatures where the pellet pressed with $0.75 \mathrm{GPa}$ at $423 \mathrm{~K}$ and $1 \mathrm{GPa}$ at $423 \mathrm{~K}$ both achieve an electrical resistivity of $\sim 36 \mu \Omega \mathrm{m}$ at $985 \mathrm{~K}$. If suffices to say that 
by lowering the pressing pressure from $1 \mathrm{GPa}$ to $0.75 \mathrm{GPa}$ and maintaining the hot pressing parameters there was very little variation. This is evident in figure 40 where cross sectional SEM images were taken from fractured pellets. This shows little or no change in texture or alignment from the sample pressed at $1 \mathrm{GPa}$ image (d) to the one pressed at $0.75 \mathrm{GPa}$ image (e).
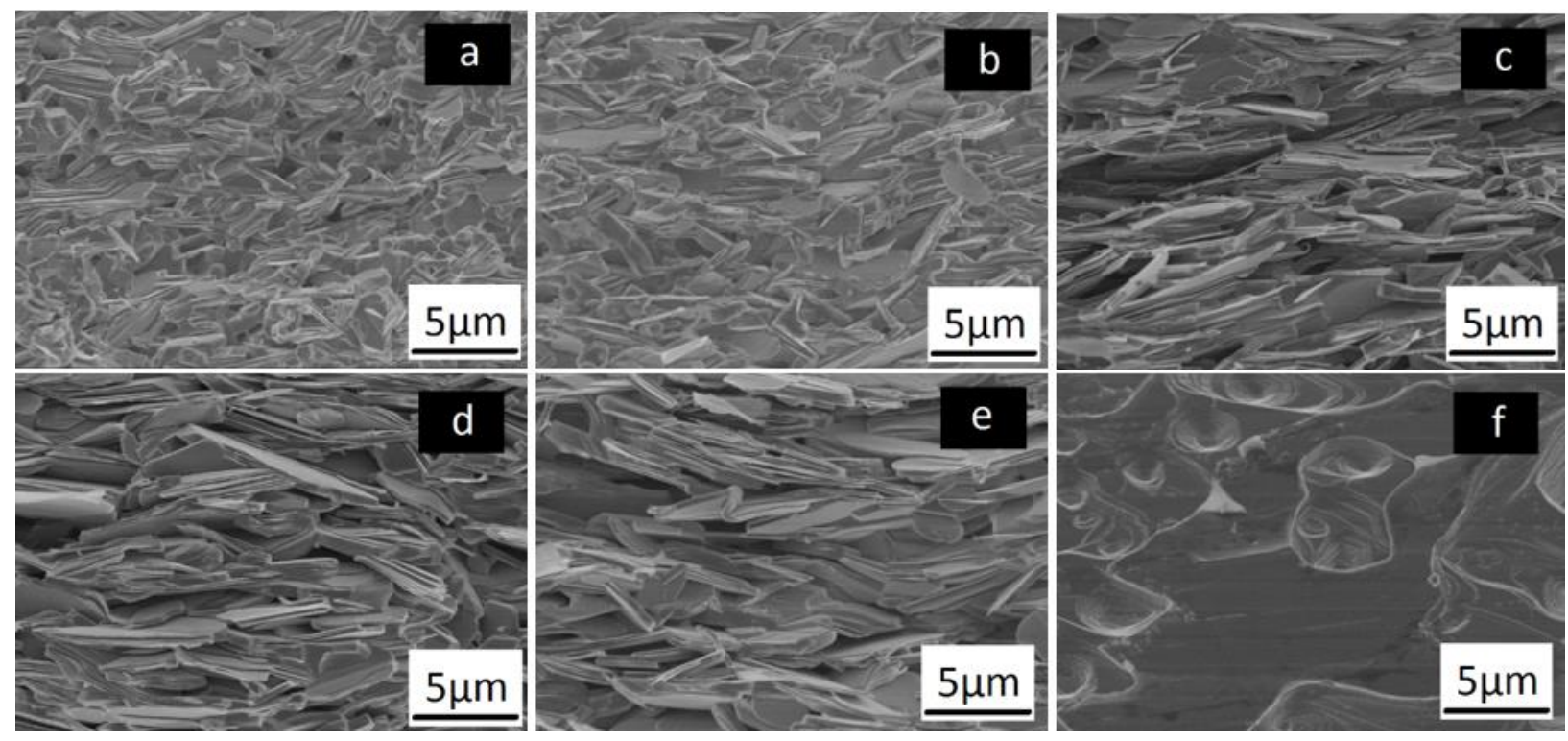

Figure 40 SEM cross-sectional view of undoped $\mathrm{Ca}_{3} \mathrm{Co}_{4} \mathrm{O}_{9}$ and doped $\mathrm{Ca}_{3} \mathrm{Bi}_{4} \mathrm{CO}_{4} \mathrm{O}_{9}$ with $u=0.0,0.1,0.2,0.3,0.3(0.75 \mathrm{GPa})$, and 0.35 .

The Absolute Seebeck coefficient is very much an intrinsic value meaning there were no changes to the Seebeck coefficient since the chemistries were not varied in any way. Since the samples' Seebeck coefficients do not change the power factor will solely depend on the variances in the samples' electrical resistivities. Therefore the three highest power factors are found in the two samples that were pressed at $1 \mathrm{GPa}$ and the one hot pressed with $0.75 \mathrm{GPa}$ of pressure; all having a power factor ranging from 0.9$0.93 \mathrm{~mW} / \mathrm{mK}^{2}$ at $973 \mathrm{~K}$ which can be seen from figure 41 . 


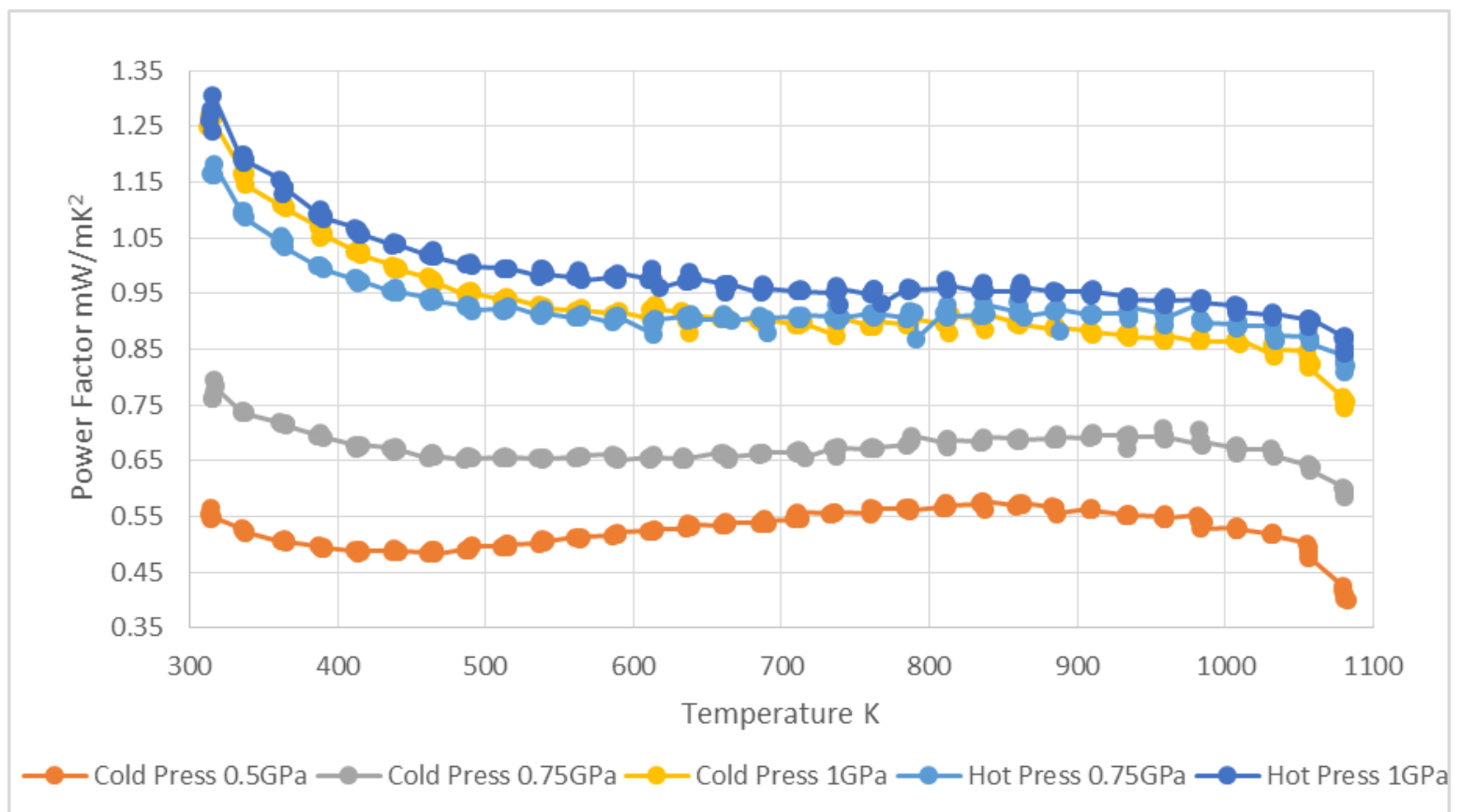

Figure 41 Temperature dependence of power factor for $\mathrm{Ca}_{3} \mathrm{Bi}_{0.3} \mathrm{Co}_{4} \mathrm{O}_{9}$ bismuth nonstoichiometric addition with pressing parameter variation.

Pellets were pressed for these three samples in order to analyze their thermal properties and compare their figure of merits. The figure or merit is already known for bismuth 0.3 hot pressed with $1 \mathrm{GPa}$ so that will be used as the reference for their thermal conductivities. The sample hot pressed at $0.75 \mathrm{GPa}$ is significantly lower than the two pressed at $1 \mathrm{GPa}$ shown in figure 42 . This is due to the lower pressing pressure leading to a lower density. The lower density creates porosity causing more phonon scattering events at the boundaries thus reducing the thermal conductivity. This density correlation is demonstrated again with the cold pressed sample having a lower thermal conductivity than the sample with the higher density due to hot pressing. As mentioned, the sample 
cold pressed at $1 \mathrm{GPa}$ had a density of $3.95 \mathrm{~g} / \mathrm{cm}^{3}$ and the sample hot pressed at $1 \mathrm{GPa}$ had a density of $4.2 \mathrm{~g} / \mathrm{cm}^{3}$.

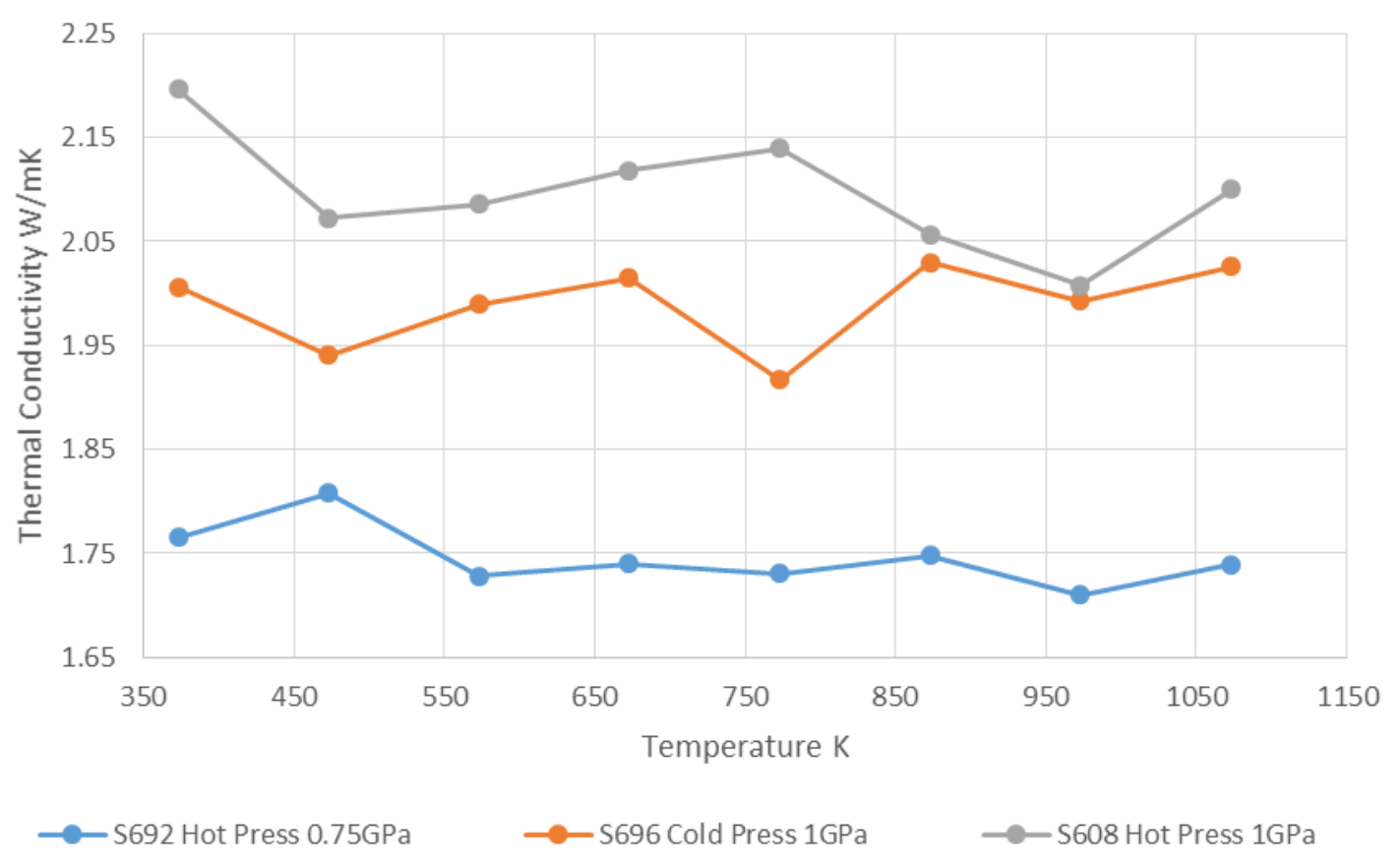

Figure 42 Temperature dependence of thermal conductivity for $\mathrm{Ca}_{3} \mathrm{Bi}_{0.3} \mathrm{Co}_{4} \mathrm{O}_{9}$ bismuth non-stoichiometric addition with pressing parameter variation.

The figure of merit of the sample with the lowest thermal conductivity was found and compared with the set discussed earlier in this chapter. Even though its electrical properties were comparative to that of hot pressed $1 \mathrm{GPa}$, the thermal conductivity of the pellet hot pressed at $0.75 \mathrm{GPa}$ is significantly lower causing it to have a higher ZT than the previous sample with the best results. Its figure of merit crests 0.5 in the high temperature regime with 0.52 at $973 \mathrm{~K}$ and $1073 \mathrm{~K}$ respectively. This 0.5 mark is a milestone as mentioned earlier since it now allows $\mathrm{Ca}_{3} \mathrm{C}_{4} \mathrm{O}_{9}$ to be competitive with materials such as SiGe. These results are shown in figure 43. 


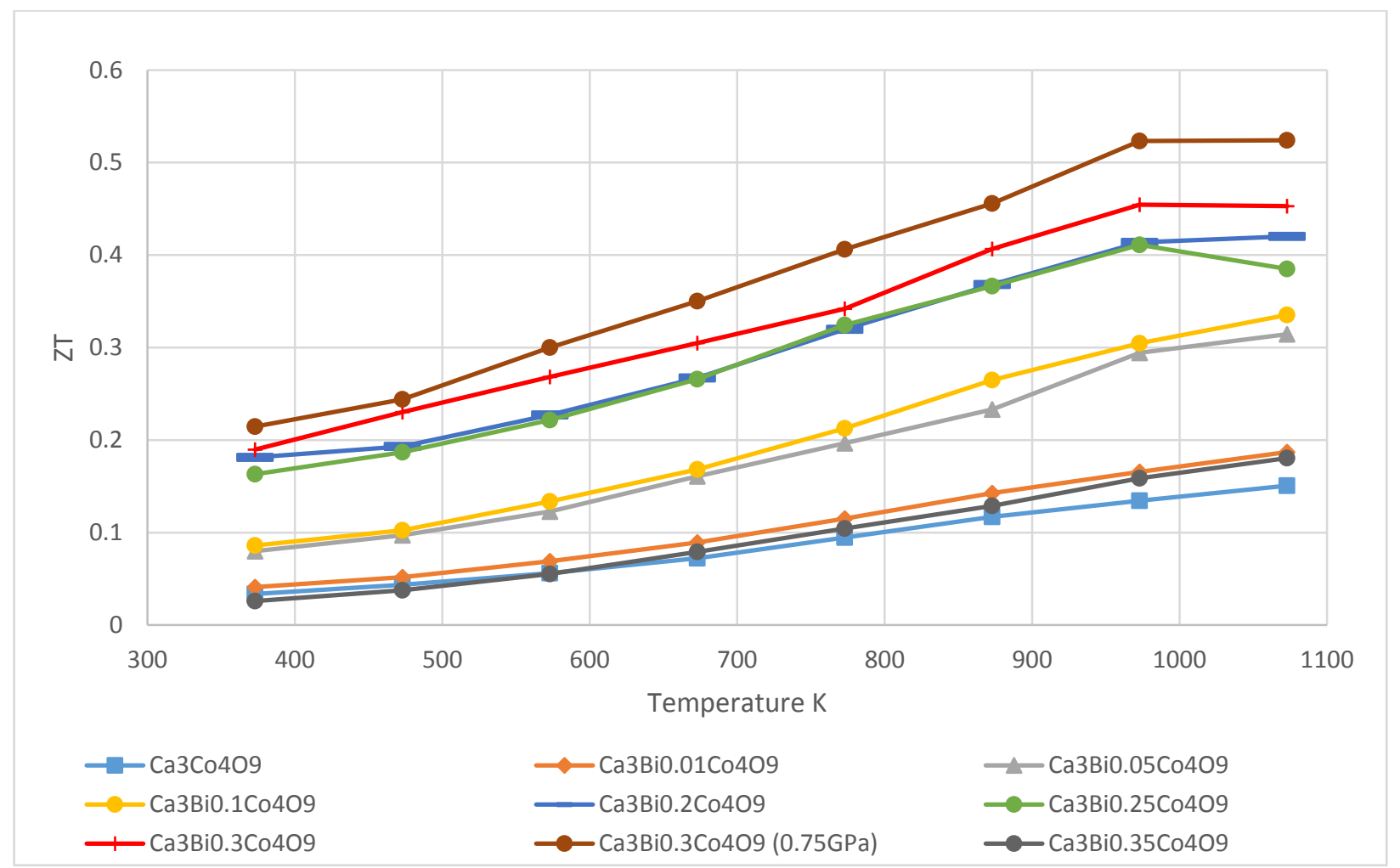

Figure 43 Temperature dependence of figure of merit for $\mathrm{Ca}_{3} \mathrm{Bi}_{0.3} \mathrm{Co}_{4} \mathrm{O}_{9}$ bismuth nonstoichiometric addition with pressing parameter variation.

\subsection{Summary of results for bismuth non-stoichiometric addition of $\mathrm{Ca}_{3} \mathrm{Co}_{4} \mathrm{O}_{9}$}

By comparing the optimal $\mathrm{Ca}_{3} \mathrm{Bi}_{0 .} \mathrm{C}_{04} \mathrm{O}_{9}$ bismuth non-stoichiometric addition results to the optimal $\mathrm{Ca}_{2.8} \mathrm{Bi}_{0.2} \mathrm{CO}_{4} \mathrm{O}_{9}$ bismuth cation substitution results it is evident that with this particular dopant, addition proves to be the preferred method. Bismuth deposits at the grain interior thus improving the crystal texture and aiding in grain growth along with segregating at the grain boundary. This is the first time the grain boundary segregation has been observed and investigated. It was shown from the X-Ray Diffraction of the samples' powders that bismuth enters the lattice and increases the lattice parameters upon using the non-stoichiometric addition method. 
After varying pressing parameters bismuth's optimization was found at the bismuth 0.3 concentration with the pressing parameters set to $0.75 \mathrm{GPa}$ for 40 minutes at $423 \mathrm{~K}$. This particular sample achieves a power factor of $\sim 0.90 \mathrm{~mW} / \mathrm{mK}^{2}$ and $\sim 0.81 \mathrm{~mW} / \mathrm{mK}^{2}$ and a thermal conductivity of $1.71 \mathrm{~W} / \mathrm{mK}$ and $1.74 \mathrm{~W} / \mathrm{mK}$ both at 973 and $1073 \mathrm{~K}$ respectively. This allows for a high dimensionless figure of merit of 0.52 at $973 \mathrm{~K}$ and $1073 \mathrm{~K}$ respectively, which are both higher than any reportings on any polycrystalline dopants. At bismuth 0.35 the sample becomes over doped and its electrical properties suffer significantly causing over a $60 \%$ decrease in its dimensionless figure of merit. Figure 44 shows a drawing to demonstrate where the bismuth deposits and how it effects the grain alignment. The light red shows the presence of bismuth in the grains and the dark red line indicates the bismuth enriched grain boundaries.

The motivation behind this set was to segregate at the grain boundary exclusively. As this chapter has detailed this was not the case. Due to the ionic size of bismuth being as close as it is to that of calcium, it was able to replace any calcium deficiencies thus doping the lattice. This prompts the idea that in order to assure the dopant will segregate at the grain boundary it is crucial to choose a dopant with a significantly large enough ionic radius that under no circumstance will it have the ability to replace the calcium ions. This sparked the motivation to use barium as a dopant in the next chapter considering barium has an ionic radius of $149 \mathrm{pm}$ compared with calcium's ionic radius of $117 \mathrm{pm}$. 
a)

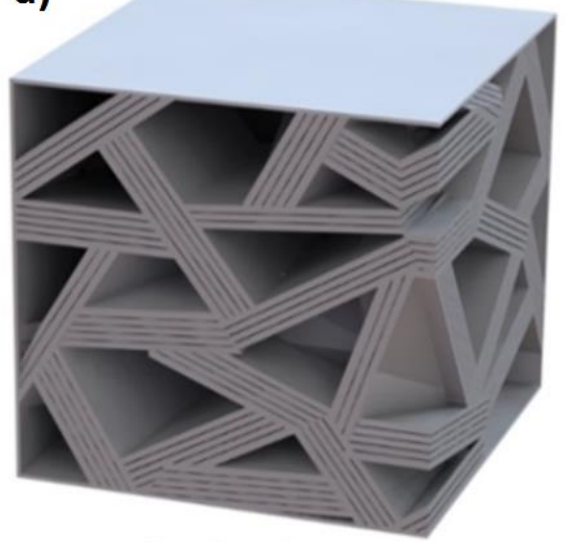

$\mathrm{Ca}_{3} \mathrm{Co}_{4} \mathrm{O}_{9}$ b)

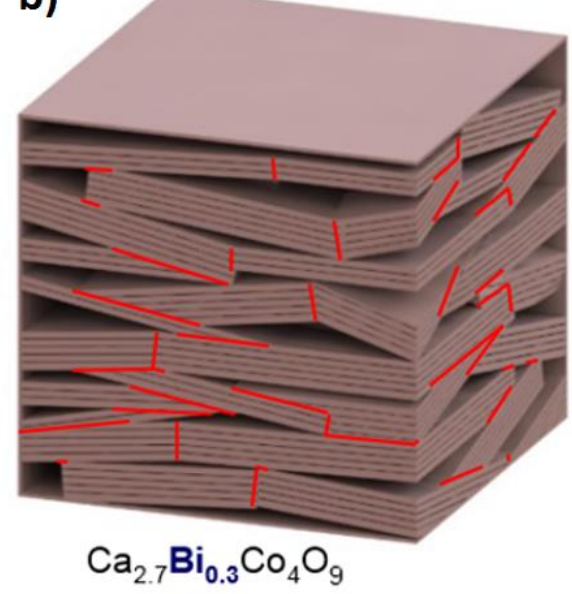

Bismuth in grain interior

Bismuth at grain boundary

Figure 44 Microstructure drawing of undoped $\mathrm{Ca}_{3} \mathrm{Co}_{4} \mathrm{O}_{9}$ and $\mathrm{Ca}_{2.7} \mathrm{Bi}_{0.3} \mathrm{Co}_{4} \mathrm{O}_{9}$. 


\subsection{References}

1 Marat Gunes, Macit Ozenbas. Effect of grain size and porosity on phonon scattering enhancement of $\mathrm{Ca}_{3} \mathrm{C}_{4} \mathrm{O}_{9}$. Journal of Alloys and Compounds Vol.626, pp.360-367, 2015.

2 Boyle, Cullen; Calvillo, Paulo; Chen, Yun; Barbero, Ever J.; Mclntyre, Dustin. Grain boundary segregation and thermoelectric performance enhancement of bismuth doped calcium cobaltite. Journal of the European Ceramic Society 36(3):601-607 Elsevier 2016 0955-2219

3 Masashi Mikami, Kanju Chong, Yuzuru Miyazaki, Tsuyoshi Kajitani, Takahiro Inoue, Satoshi Sodeoka, and Ryoji Funahashi. Bi-Substitution effects on crystal structure and thermoelectric properties of $\mathrm{Ca}_{3} \mathrm{C}_{4} \mathrm{O}_{9}$ single crystals. Japanese Journal of Applied Physics. Vol.45, No.5A, pp.4131-4136, 2006.

${ }^{4}$ K. Rubešová, T. Hlásek, V. Jakeš, Š. Huber, J. Hejtmánek, D. Sedmidubský. Effect of a powder compaction process on the thermoelectric properties of $\mathrm{Bi}_{2} \mathrm{Sr}_{2} \mathrm{Co}_{1.8} \mathrm{O}_{x}$ ceramics. Journal of the European Ceramic Society, Vol.35, pp.525-531, 2015.

5 Sajid Butt, Yaoyu Ren, Muhammad Umer Farooq, Bin Zhan, Rizwan ur Rahman Sagar, Yuanhua Lin, Ce-Web Nan. Enhanced thermoelectric performance of heavy-metals ( $M$ : $\mathrm{Ba}, \mathrm{Pb})$ doped misfit-layered ceramics: $\left(\mathrm{Ca2}-\mathrm{x}_{\mathrm{x}} \mathrm{CoO}_{3}\right)_{0.62}\left(\mathrm{CoO}_{2}\right)$. Energy Conversion and Management. Vol.83, pp.35-41, 2014.

6 Calvillo, Paulo. Chen, Yun. Boyle, Cullen. Barnes, Paul. N. Song, Xueyan. Thermoelectric performance enhancement of calcium cobaltite through barium grain boundary segregation. Inorganic Chemistry. American Chemical Society Publications. September 11, 2015.

7 Tao Wu, Trevor A. Tyson, Haiyan Chen, Jianming Bai, Hsin Wang and Cherno Jaye. A structural phase transition in $\mathrm{Ca}_{3} \mathrm{Co}_{4} \mathrm{O}_{9}$ Associated with enhanced high temperature thermoelectric properties. Cornell University Library. April 15, 2012.

8 T. Takeuchi, T. Kondo, K. Soda, U. Mizutani, R. Funahashi, M. Shikano, S. Tsuda, T. Yokoya, S. Shin, T. Muro. Electronic structure and large thermoelectric power in $\mathrm{Ca}_{3} \mathrm{CO}_{4} \mathrm{O}_{9}$. Journal of Electron Spectroscopy and Related Phenomena. Vol.137-140, pp.595-599, 2004.

9 T. Sun, H. H. Hng, Q. Y. Yan, and J. Ma. Enhanced high temperature thermoelectric properties of $\mathrm{Bi}$-doped c-axis oriented $\mathrm{Ca}_{3} \mathrm{C}_{4} \mathrm{O}_{9}$ thin films by pulsed laser deposition. Journal of Applied Physics. Vol.108, 2010.

${ }^{10}$ Guangkun Ren, Jinle Lan, Chengcheng Zeng, Yaochun Liu, Bin Zhan, Sajid Butt, YuanHua Lin, and Ce-Wen Nan. High performance oxides-based thermoelectric materials. Journal of Minerals, Metals \& Materials Society. Vol.67, No.1, pp.211-221, 2015.

11 Ruoming Tian, Tianshu Zhang, Dewei Chu, Richard Donelson, Li Tao, Sean Li. Enhancement of high temperature thermoelectric performance in $\mathrm{Bi}, \mathrm{Fe}$ co-doped layered oxide-based material $\mathrm{Ca}_{3} \mathrm{C}_{4} \mathrm{O}_{9+\delta}$. Journal of Alloys and Compounds. Vol.615, pp.311-315, 2014. 
12 Susumu Fujii and Masato Yoshiya. Manipulating thermal conductivity by interfacial modification of misfit-layered cobaltites $\mathrm{Ca}_{3} \mathrm{CO}_{4} \mathrm{O}_{9}$. Journal of Electronic Materials, Vol.45, No.3, pp.1217-1226, 2016

${ }^{13}$ Lijun Wu, Qingping Meng, Christian Jooss, Jin-Cheng Zheng, H. Inada, Dong Su, Qiang $\mathrm{Li}$, and Yimei Zhu. Origin of phonon glass-electron crystal behavior in thermoelectric layered cobaltite. Advanced Functional Materials. Vol.23, pp.5728-5736, 2013.

${ }^{14}$ Chong Xiao, Jie Xu, Boxiao Cao, Kun Li, Mingguang Kong, and Yi Xie, Solid-solutioned homojunction nanoplates with disordered lattice: a promising approach toward "phonon glass electron crystal" thermoelectric materials. Journal of the American Chemical Society. Vol.134, pp.7971-7977, 2012.

${ }^{15}$ Haoshan Hao, Limin zhao, and Xing Hu. Microstructure and thermoelectric properties of $\mathrm{Bi}$ - and $\mathrm{Cu}$-substituted $\mathrm{Ca}_{3} \mathrm{C}_{4} \mathrm{O}_{9}$ oxides. Journal of Materials Science Technology., Vol.25, No.1, pp.105-108, 2009.

${ }^{16}$ G. Constantinescu, Sh. Rasekh, M. A. Torres, M. A. Madre, A. Sotelo, J. C. Diez. Improvement of thermoelectric properties in $\mathrm{Ca}_{3} \mathrm{Co}_{4} \mathrm{O}_{9}$ ceramics by $\mathrm{Ba}$ doping. Journal of Materials Science., Vol.26, pp.3466-3473, 2015.

17 Majid Abdellahi, Hamid Ghayour, Maryam Bahmanpour. Effect of process parameters and synthesis method on the performance of thermoelectric ceramics: A novel simulation. Ceramics International. Vol.41, pp.6991-6998, 2015.

18 Kasper A. Borup, Johannes de Boor, Heng Wang, Fivos Drymiotis, Franck Gascoin, Xun Shu, Lidong Chen, Mikhail I. Fedorov, Eckhard Müller, Bo B. Iverson, and G. Jeffrey Snyder. Measuring thermoelectric transport properties of materials. Energy \& Environmental Science. Vol.8, pp.423-435, 2015.

19 Haoshan Hao, Qinglin He, Changqing Chen, Hongwei Sun, and Xing Hu. Textured structure and anisotropic thermoelectric properties of $\mathrm{Ca}_{2.7} \mathrm{Bi}_{0.3} \mathrm{C}_{0} \mathrm{O}_{9}$ oxide prepared by conventional solid-state reaction. International Journal of Modern Physics B, Vol.23, No.1, pp.87-95, 2009.

20 M. Sabarinathan, M. Omprakash, S. Harish, M. Navaneethan, J. Archana, S. Ponnusamy, H. Ikeda, T. Takeuchi, C. Muthamizhchelvan, Y. Hayakawa. Enhancement of power factor by energy filtering effect in hierarchical $\mathrm{BiSbTe}_{3}$ nanostructures for thermoelectric applications. Applied Surface Science. Vol418, pp.246-251, 2017.

${ }^{21}$ Zhi-Lei Wang, Takahiro Akao, Tetsuhiko Onda, Zhong-Chun Chen. Microstructure and thermoelectric properties of Bi-Sb-Te bulk materials fabricated from rapidly solidified powders. Scripta Materialia. Vol.136, pp.111-114, 2017.

${ }^{22}$ Kenji Tanabe, Ryuji Okazaki, Hiroki Taniguchi, and Ichiro Terasaki. Optical conductivity of layered calcium cobaltate $\mathrm{Ca}_{3} \mathrm{C}_{4} \mathrm{O}_{9}$. Journal of Physics: Condensed Matter. Vol.28, 2016.

${ }^{23}$ Zunyi Tian, Xiaohong Wang, Jun Liu, Zhongjin Lin, Yangsen Hu, Yigui Wu, Chao Han, Zhiyu Hu. Power factor enhancement induced by $\mathrm{Bi}$ and $\mathrm{Mn}$ co-substitution in $\mathrm{Na}_{x} \mathrm{CoO}_{2}$ thermoelectric materials. Journal of Alloys and Compounds. Vol.661, pp.161=167, 2016. 
${ }^{24}$ Siwen Li, Ryoji Funahashi, Ichiro Matsubara, Kazuo Ueno, Satoshi Sodeoka, and Hiroyuki Yamada. Synthesis and Thermoelectric Properties of the New Oxide Materials $\mathrm{Ca}_{3-x} \mathrm{Bi}_{x} \mathrm{Co}_{4} \mathrm{O}_{9+\delta}(0.0<\mathrm{x}<0.75)$. American Chemical Society. Chemistry of Materials. Vol.12, pp.2424-2427, 2000.

${ }^{25}$ Ankam Bhaskar, Z.-R. Lin, Chia-Jyi Liu. Thermoelectric properties of Ca2.95Bio.05C04${ }_{x} \mathrm{Fe}_{\times} \mathrm{O}_{9+\delta}(0<\mathrm{x}<0.15)$. Energy Conversion and Management Vol.76, pp. 63-67, 2013. 


\section{Chapter 6: \\ Non-stoichiometric Addition of Barium to Enhance Electrical Power Factor of $\mathrm{Ca}_{3} \mathrm{CO}_{4} \mathrm{O}_{9}$}

\subsection{Background and motivation for Barium as a dopant in $\mathrm{Ca}_{3} \mathrm{Co}_{4} \mathrm{O}_{9}$}

Barium was identified in 1774 but it wasn't until 1808 that English chemist Sir Humphry Davy was able to isolate it through electrolysis. ${ }^{[1]}$ Like most elements barium was named after a Greek word and due to its high mass it was named after the word barys, meaning heavy. ${ }^{[1]}$ Barium is considered one of the alkaline-earth metals and is soft and metallic. ${ }^{[2]}$ It reacts with the oxygen in air, forming barium oxide, $\mathrm{BaO}$, which is why it is never found free in nature. ${ }^{[1]}$ Barium has also been reported on in the past by Zhang et al. and Lu et al. stating that barium has improved the thermoelectric properties by decreasing their electrical resistivities and thermal conductivities. ${ }^{[3]}$ Barium is also known to have a very low electrical resistivity. Zhang et al. reported a ZT of 0.2 at $973 \mathrm{~K}$ for $\mathrm{Ca}_{3} \mathrm{C}_{4} \mathrm{O}_{9}$ doped with barium using the cation substitution method. ${ }^{[4]}$ Using the cation substitution as well, Constantinescu et al. reported a power factor of $0.29 \mathrm{~mW} / \mathrm{mK}^{2}$ for barium doped $\mathrm{Ca}_{3} \mathrm{Co}_{4} \mathrm{O}_{9} .{ }^{[5]}$ All reports on barium to date utilize the cation substitution method. This chapter details the investigation of doping $\mathrm{Ca}_{3} \mathrm{C}_{4} \mathrm{O}_{9}$ with barium by means of the non-stoichiometric addition method in order to segregate the grain boundary knowing that barium's $149 \mathrm{pm}$ ionic radius will not allow it to deposit itself in place of the 114 pm calcium ions should there be any vacancies. 


\subsection{Experimental Procedure}

$\mathrm{Ca}_{3} \mathrm{Ba}_{4} \mathrm{C}_{4} \mathrm{O}_{9}$ powders where $\mathrm{u}=0.0,0.03,0.05$, and 0.07 were obtained through a sol-gel route in which non-stoichiometric ratios of $\mathrm{Ca}\left(\mathrm{NO}_{3}\right)_{2} \bullet 4 \mathrm{H}_{2} \mathrm{O}, \mathrm{Co}\left(\mathrm{NO}_{3}\right)_{2} \cdot 6 \mathrm{H}_{2} \mathrm{O}$ and $\mathrm{Ba}\left(\mathrm{NO}_{3}\right)_{2}$ were mixed in deionized water. Ethylene glycol and polyethylene glycol were used to aid polymerization of the solution and vary grain size. ${ }^{[6]}$ Lastly, nitric acid was added to induce nitrate salts decomposition and facilitate new compound formation. ${ }^{[7]}$ The liquid solution was then submerged in a silicone oil bath on a hot plate and mechanically stirred at $353 \mathrm{~K}$ for 3 hours to achieve the sol-gel state. The sol-gel was then distributed to ceramic crucibles loosely covered with aluminum foil and put into a box furnace heated to $773 \mathrm{~K}$ with a ramp rate of $10 \mathrm{~K}$ per minute and held for 2 hours before being cooled at a rate of $10 \mathrm{~K}$ per minute. Holding the temperature here, at $773 \mathrm{~K}$, for the allotted time will ensure that any inorganic material is removed from the material. The ashes were then suspended in ethanol and ball milled for 20 minutes alternating between 1 minute of milling and 1 minute of resting in order to aid in the dissipation of heat. The powders were deposited in a tubular furnace where they were calcined in an oxygen rich induced environment with a ramp rate of $10 \mathrm{~K}$ per minute and held at $973 \mathrm{~K}$ for 4 hours, to achieve the $\mathrm{Ca}_{3} \mathrm{Co}_{4} \mathrm{O}_{9}$ crystal lattice. The powders were then cooled down to room temperature at a rate of $10 \mathrm{~K}$ per minute.

Two pellets were pressed, one to test its electrical properties and the other for its thermal properties. The calcined powders were pressed uniaxially with $1 \mathrm{GPa}$ of force and held for 10 minutes at $298 \mathrm{~K}$. The pellets' densities were obtained before they were put in to a tubular furnace to be sintered. The sintering process removes any extra moisture the pellet may contain and assures proper grain alignment. Pellets were sintered 
in an oxygen rich induced environment with a ramp rate of $10 \mathrm{~K}$ per minute and held at $1233 \mathrm{~K}$ for 9 hours. It was then cooled back to room temperature at a rate of $4 \mathrm{~K}$ per minute and once again the densities were taken. Pellets were cut into a $2 \mathrm{~mm} \times 3.5 \mathrm{~mm}$ x $9 \mathrm{~mm}$ rectangular shapes and inserted into a Linseis LSR 3-Seebeck machine, subjected to a low pressure helium, He, environment where the pellet's electrical resistivity and Absolute Seebeck Coefficient were simultaneously determined by means of a dc four-probe method. Since helium is an inert gas it will not react or interfere with the measurement. A second pellet was cut along a different axis and inserted into the Linseis LFA 1000 machine to test its thermal conductivity. The pellet was cut to be $2 \mathrm{~mm}$ thick and polished to a diameter of $11-13 \mathrm{~mm}$. Any less than $11 \mathrm{~mm}$ and there would be room for heat to escape the holder, any more than $13 \mathrm{~mm}$ and the pellet would be too large for the holder. The thermal conductivity is calculated by the equation

$$
K=\lambda C_{p} \rho_{m}
$$

Where $\lambda$ is the thermal diffusity in meters squared per seconds $\left(\mathrm{m}^{2} / \mathrm{s}\right), \mathrm{C}_{\mathrm{p}}$ is the specific heat capacity in joules per kilogram kelvin $(\mathrm{J} / \mathrm{kg} \cdot \mathrm{K})$, and $\rho_{\mathrm{m}}$ is the mass density in grams per centimeter cubed $\left(\mathrm{g} / \mathrm{cm}^{3}\right)$. As stated in the first section, these are the three values needed in order to obtain the materials' ZT value. For barium addition, four different chemistries were used. The first chemistry is the baseline of $\mathrm{Ca}_{3} \mathrm{C}_{4} \mathrm{O}_{9}$ and the other three are dopings of barium $0.03,0.05$, and 0.07 . 


\subsection{Effect of barium's grain boundary segregation on the power factor}

6.3.1 Increased electrical properties of $\mathrm{Ca}_{3} \mathrm{Co}_{4} \mathrm{O}_{9}$ through $\mathrm{Ba}$ addition

Low doping levels of barium demonstrate a higher electrical resistivity than the undoped sample shown in figure 45 . However, unlike the bismuth samples, the barium samples show a trend that maintains a low electrical resistivity through all temperature ranges. This causes the sample with the low doping of $\mathrm{Ba} 0.03$ to achieve a lower electrical resistivity than the undoped sample at mid and high temperature ranges. This is due to barium being an alkaline-earth metal which acts more like a semi-conductor than a metal. ${ }^{[8]}$ The $\mathrm{Ca}_{3} \mathrm{C}_{4} \mathrm{O}_{9}$ material demonstrates an immediate drop in electrical resistivity at low temperatures with higher levels of barium doping. The sample $\mathrm{Ca}_{3} \mathrm{Ba}_{0.5} \mathrm{C}_{4} \mathrm{O}_{9}$ demonstrates a room temperature resistivity of $\sim 35 \mu \Omega m$ which is less than half that of the baseline sample. As the baseline increases to almost $90 \mu \Omega \mathrm{m}$ at $1081 \mathrm{~K}$ the sample with optimal barium doping only increases to around $45 \mu \Omega m$ which once again is $50 \%$ of the baseline. 


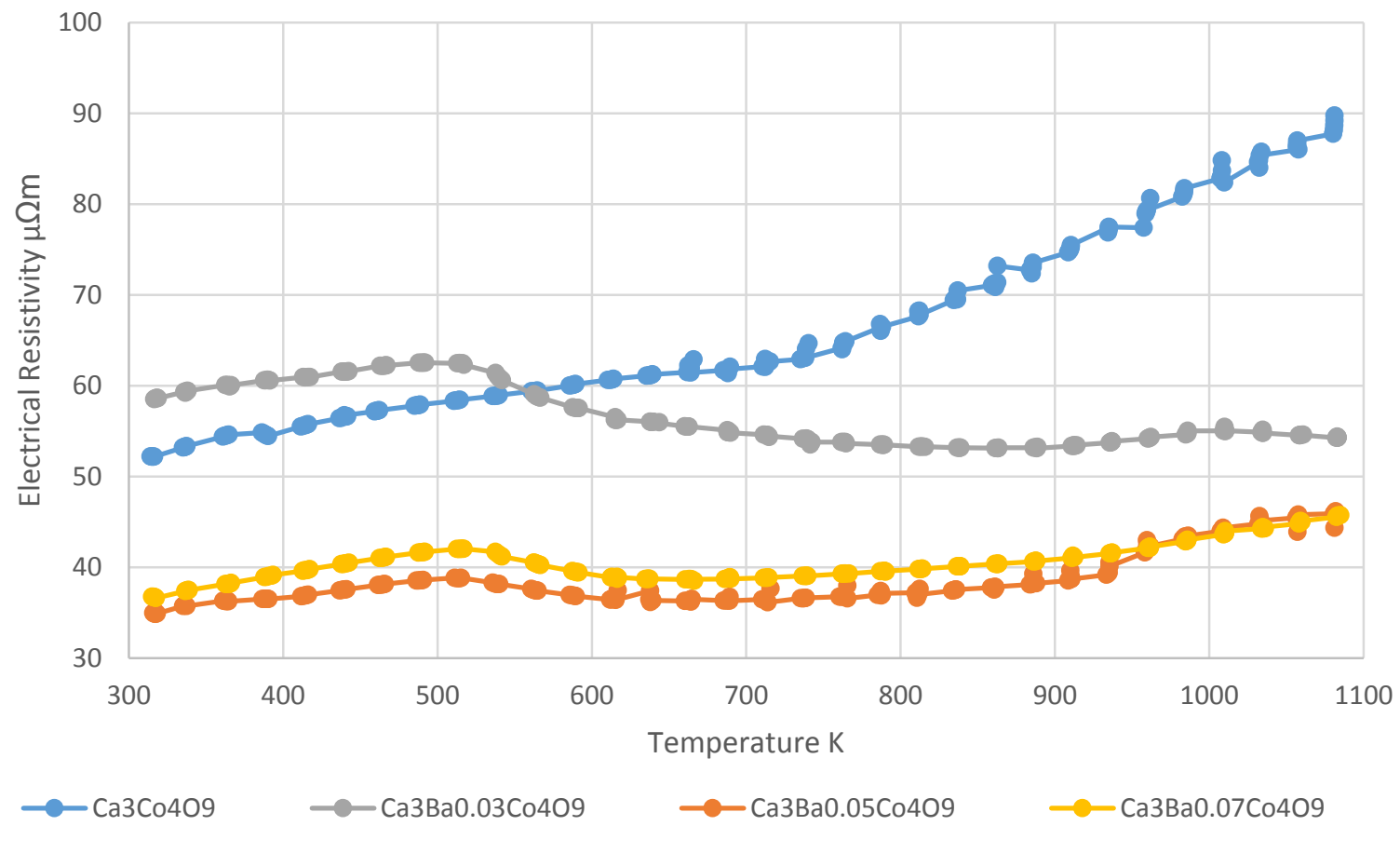

Figure 45 Temperature dependence of electrical resistivity for $\mathrm{Ca}_{3} \mathrm{Ba}_{4} \mathrm{Co}_{4} \mathrm{O}_{9}$ barium non-stoichiometric addition

Another benefit of using barium as a dopant is its ability to achieve a high Seebeck coefficient in the low to mid temperature range shown in figure 46. It was mentioned in the previous chapter that bismuth causes an increase in the Absolute Seebeck coefficient at room temperature to a saturation level around $150 \mu \mathrm{V} / \mathrm{K}$. Barium betters this by having a room temperature Absolute Seebeck coefficient just shy of $170 \mu \mathrm{V} / \mathrm{K}$, in all doping levels, which is one of the highest reported for this material. ${ }^{[9]}$ This "dramatic increase" as it was referred to by Calvillo et al. has been attributed to the barium segregating at the $\mathrm{Ca}_{3} \mathrm{C}_{4} \mathrm{O}_{9}$ grain boundaries. ${ }^{[10]}$ The barium deposited at the grain boundary acts as a filter for the carrier concentration causing the enhancement in the absolute Seebeck coefficient at room temperature. 


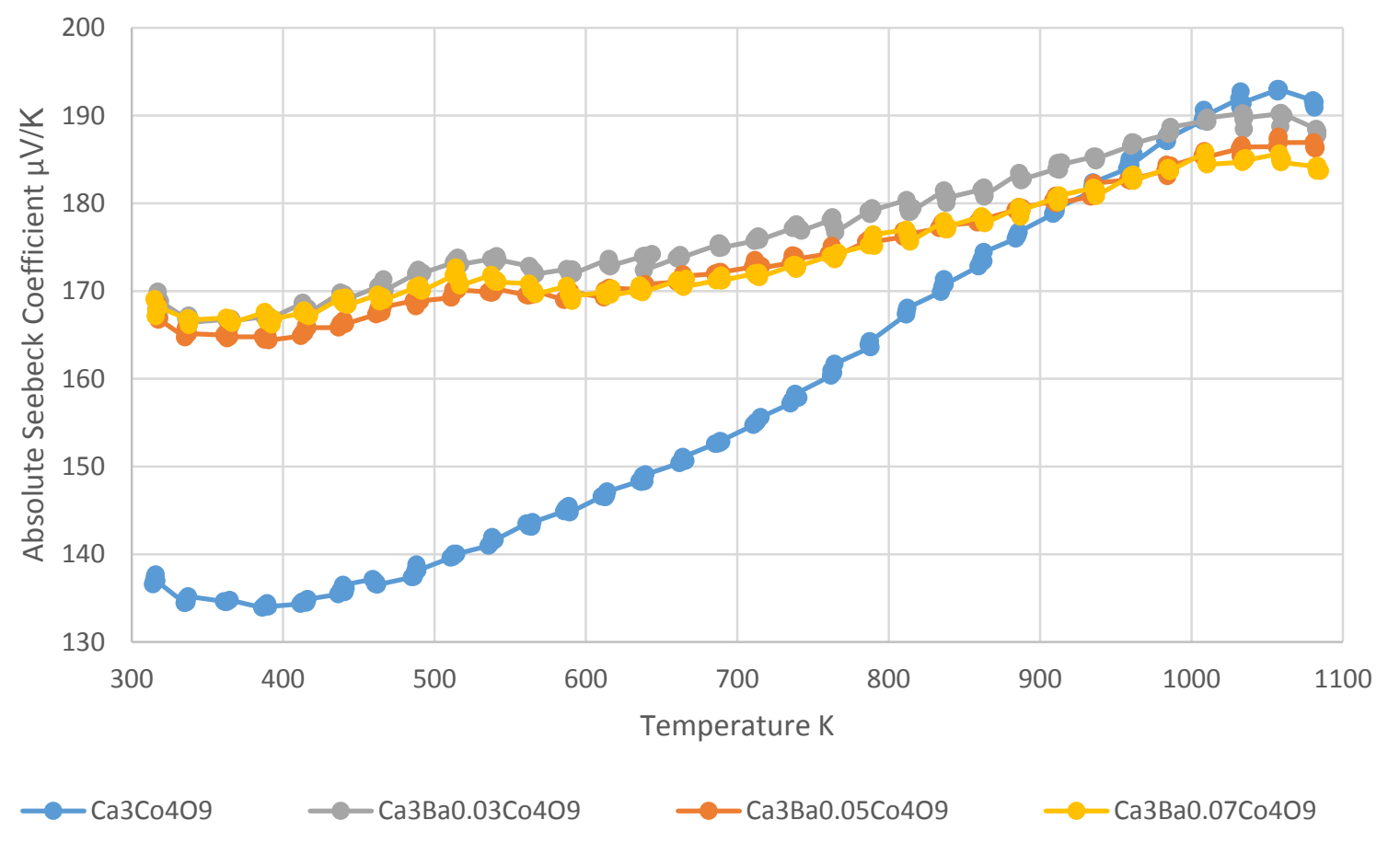

Figure 46 Temperature dependence of absolute seebeck coefficient for $\mathrm{Ca}_{3} \mathrm{Ba}_{4} \mathrm{Co}_{4} \mathrm{O}_{9}$ barium non-stoichiometric addition.

The low electrical resistivity of $\sim 35 \mu \Omega \mathrm{m}$ coupled with the high Absolute Seebeck coefficient of $167 \mu \mathrm{V} / \mathrm{K}$, both at $315 \mathrm{~K}$, combine to achieve a high power factor of 0.82 $\mathrm{mW} / \mathrm{mK}^{2}$ at $315 \mathrm{~K}$ demonstrated in figure 47 . These two parameters play a very similar role through all temperature ranges. The Absolute Seebeck coefficient slowly increases with temperature as the electrical resistivity slowly increases as well to obtain its highest power factor of $0.85 \mathrm{~mW} / \mathrm{mK}^{2}$ at $810 \mathrm{~K}$. Both are achieved with the same concentration of barium 0.05 and are over two times greater than the power factor of the baseline at the respective temperatures. 


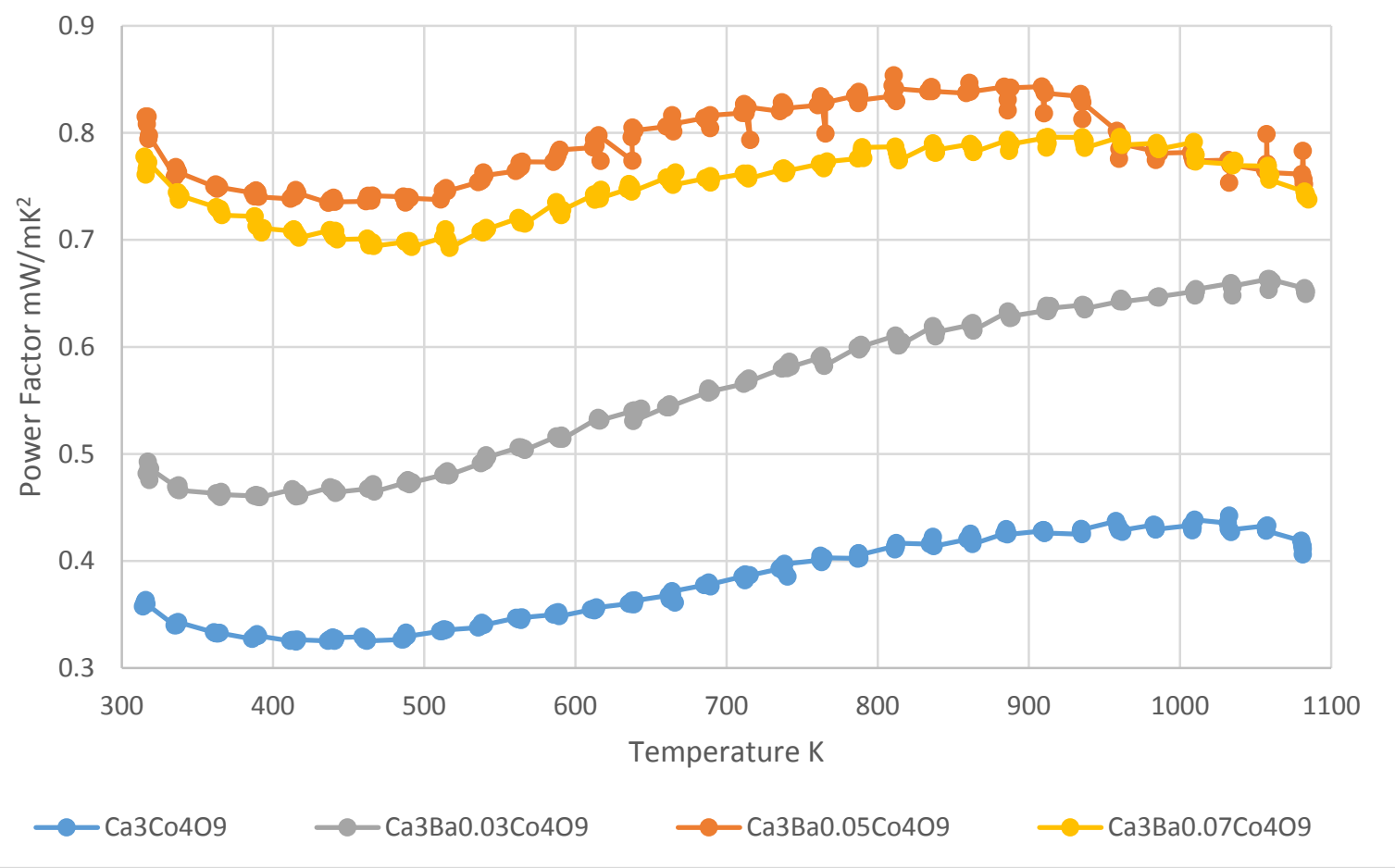

Figure 47 Temperature dependence of power factor for $\mathrm{Ca}_{3} \mathrm{Ba}_{4} \mathrm{Co}_{4} \mathrm{O}_{9}$ barium nonstoichiometric addition.

\subsubsection{Microstructure and nanostructure of Barium doped $\mathrm{Ca}_{3} \mathrm{C}_{4} \mathrm{O}_{9}$}

Figure 48 shows the cross-sectional SEM view for surface fractured pellets. As the barium doping concentration increases from image (a) to (c) the grain alignment vastly improves. Image (d) demonstrates the misalignment of grains in the over doped sample. This shows that just like bismuth, barium has the ability to improve crystal texture through grain alignment as well. The SEM image of the optimal concentration of $\mathrm{Ca}_{3} \mathrm{Ba}_{0.5} \mathrm{C}_{4} \mathrm{O}_{9}$ illustrated in image (c) of figure 48 clearly demonstrates the vastly improved grain alignment from the pure $\mathrm{Ca}_{3} \mathrm{Co}_{4} \mathrm{O}_{9}$ illustrated in image (a). However, unlike bismuth, barium does not trigger near as much grain growth allowing the grains to compact more easily giving it a much higher density than previous chemistry sets. The optimum bismuth 
cation substitution density was $3.95 \mathrm{~g} / \mathrm{cm}^{3}$, the bismuth non-stoichiometric addition density was $4.20 \mathrm{~g} / \mathrm{cm}^{3}$ whereas the density of the optimal barium concentration is 4.51 $\mathrm{g} / \mathrm{cm}^{3}$.
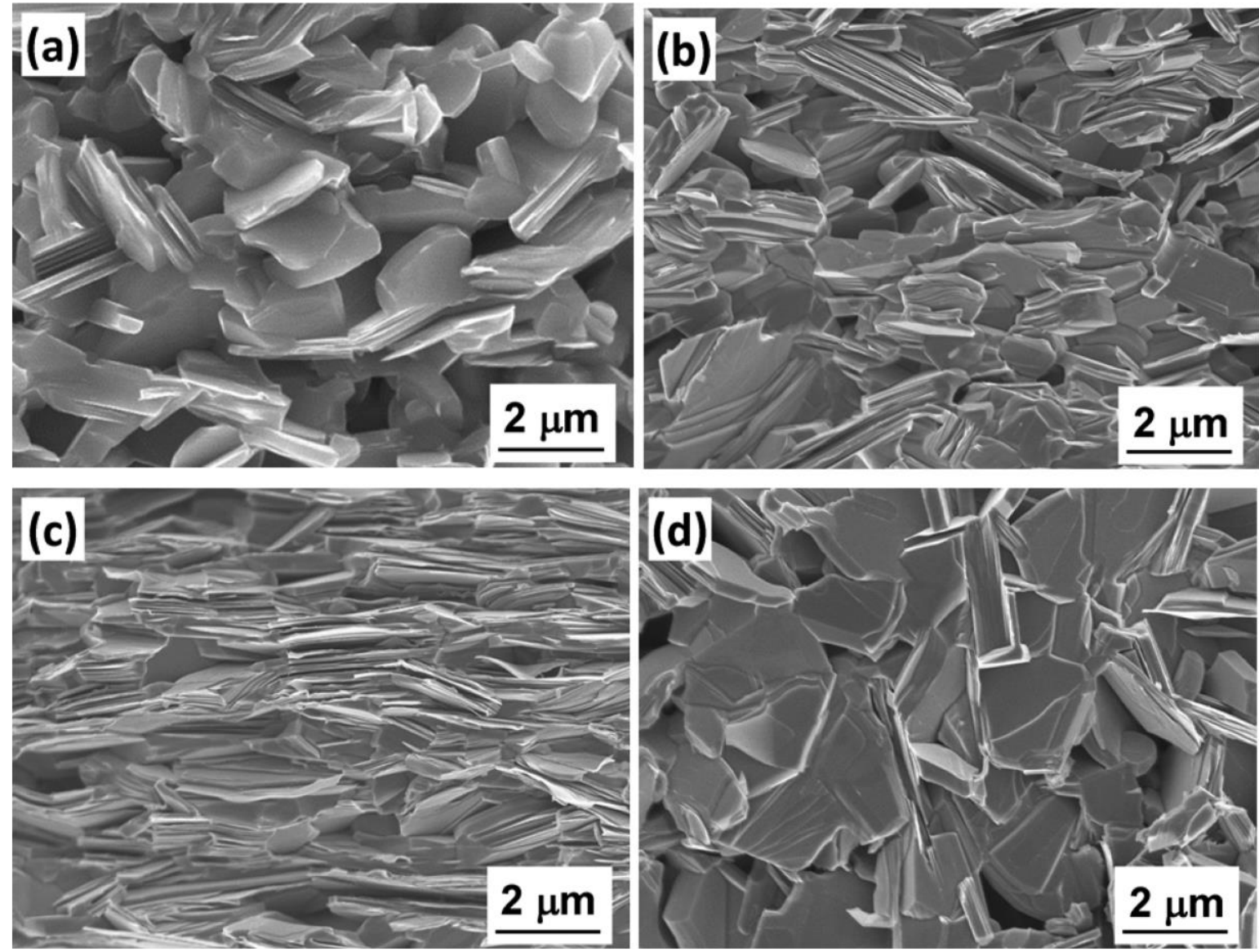

Figure 48 SEM cross-sectional view of undoped $\mathrm{Ca}_{3} \mathrm{Co}_{4} \mathrm{O}_{9}$ and doped $\mathrm{Ca}_{3} \mathrm{Ba}_{4} \mathrm{Co}_{4} \mathrm{O}_{9}$ with $u=0.0,0.01,0.05$, and 0.1. ${ }^{[10]}$

The samples were investigated by use of a transmission electron microscope TEM as can be seen in figure 49. Image (a) shows two grains $A$ and $B$ where 12 different EDS data points were taken. For this image a low magnification TEM image was taken. Table 2 details the atom percentage at each location demonstrating proof that barium is not found at locations 1-4 and 9-12 which were all taken from inside the grain itself. In fact, barium only deposits within the grain boundary which reinforces its ability to segregate at 
the boundary. Image (b) is a high resolution TEM image showing that there was no secondary phase that had formed at the grain boundary. Images (c) and (e) show the electron diffraction collected from grain $A$ and $B$ respectively while image (d) shows the electron diffraction collected at the grain boundary between grains $\mathrm{A}$ and $\mathrm{B}$.

(a)
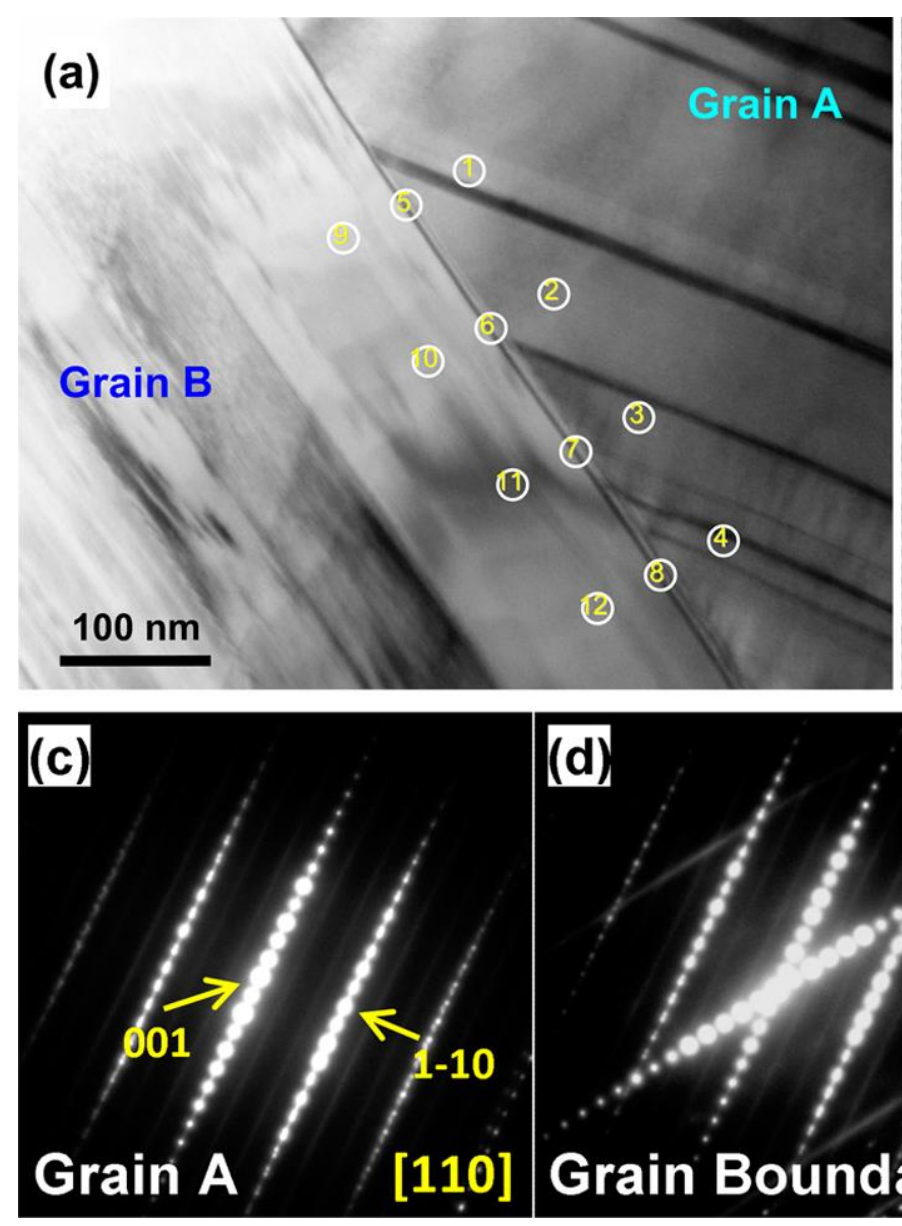

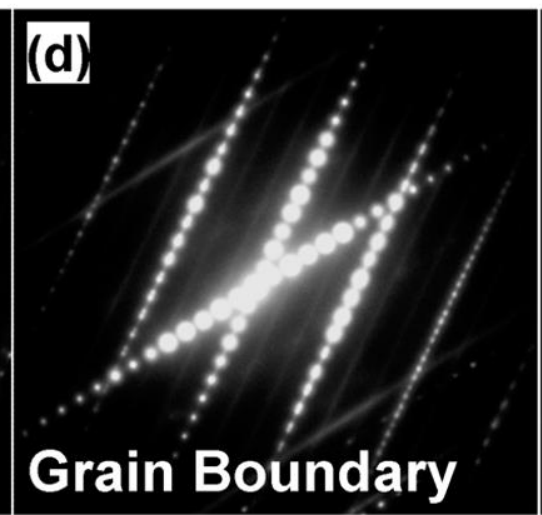

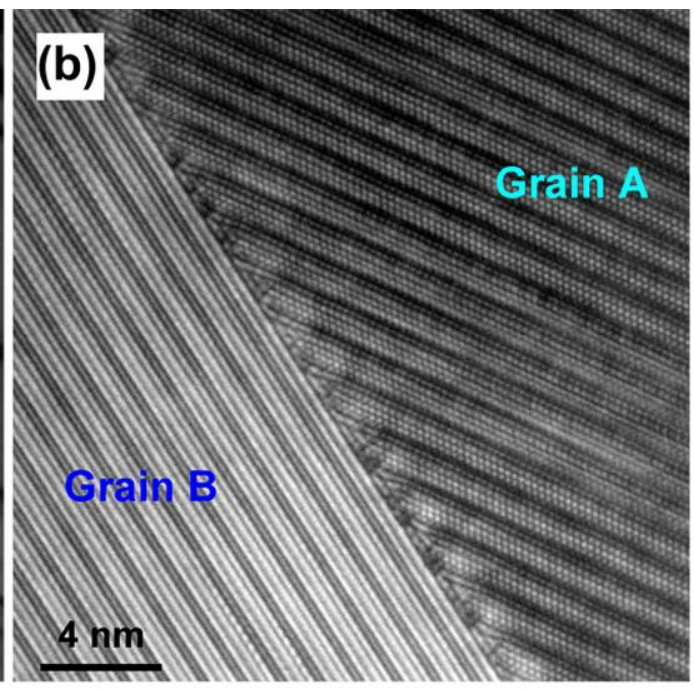

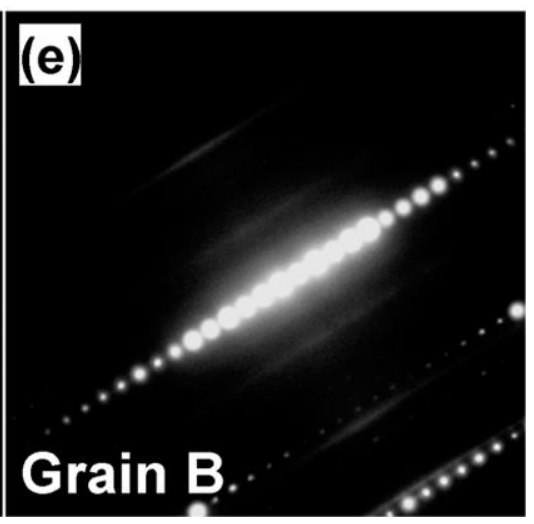

Figure 49 Diffraction contrast images and the selected area diffraction patterns from the $\mathrm{Ca}_{3} \mathrm{Ba}_{0.05} \mathrm{CO}_{4} \mathrm{O}_{9}$ sample. ${ }^{[10]}$ 
Table 2 Chemical Compositions of two neighboring grains $A$ (spots 1,2, 3, and 4) and $B$ (spots 9, 10, 11, and 12) and at the grain boundary between (spots 5, 6, 7, and 8) shown in figure 42. [10]

\begin{tabular}{|lccccccccccccc|}
\hline atom \% & 1 & 2 & 3 & 4 & 5 & 6 & 7 & 8 & 9 & 10 & 11 & 12 \\
$\mathrm{OK}$ & 59.53 & 56.92 & 61.78 & 67.80 & 62.48 & 61.64 & 61.26 & 58.52 & 63.34 & 61.06 & 59.85 & 57.99 \\
$\mathrm{CaK}$ & 17.54 & 20.57 & 17.29 & 13.74 & 15.70 & 16.31 & 16.86 & 18.55 & 16.08 & 17.30 & 17.74 & 18.63 \\
$\mathrm{CoK}$ & 22.93 & 22.51 & 20.93 & 18.46 & 21.42 & 21.75 & 21.73 & 22.64 & 20.59 & 21.64 & 22.41 & 23.38 \\
$\mathrm{Ba}$ & 0 & 0 & 0 & 0 & 0.40 & 0.30 & 0.15 & 0.29 & 0 & 0 & 0 & 0 \\
\hline
\end{tabular}

\subsection{Summary of electrical properties of Barium non-stoichiometric addition of $\mathrm{Ca}_{3} \mathrm{CO}_{4} \mathrm{O}_{9}$}

Barium has proven to be a great candidate for a thermoelectric material based on these results and the ones from the literature. Barium's ability to deposit itself at the grain boundary and enhance the Absolute Seebeck coefficient while maintaining a low resistivity in all temperature ranges vastly improves the efficiency of the material. This is the first reporting on barium doping using the non-stoichiometric addition method. The non-stoichiometric addition method also proves beneficial despite not preserving the stoichiometry. Furthermore, there were no secondary phases present at the grain boundary despite this lack of stoichiometric preservation. This method in combination with barium's ionic size help to assure that barium will deposit itself at the grain boundary in order to create this segregation to enhance the Absolute Seebeck coefficient. This allows barium doped $\mathrm{Ca}_{3} \mathrm{Co}_{4} \mathrm{O}_{9}$ to have enhanced electrical properties and achieve a power factor of $0.82 \mathrm{~mW} / \mathrm{mK}^{2}$ at $315 \mathrm{~K}$ and $0.84 \mathrm{~mW} / \mathrm{mK}^{2}$ at $910 \mathrm{~K}$, proving to hold great promise in this field. Figure 50 shows a drawing to demonstrate where the barium deposits and 
how it effects the grain alignment. Notice there is no presence of barium at the grain interior only at the grain boundary indicated in yellow.

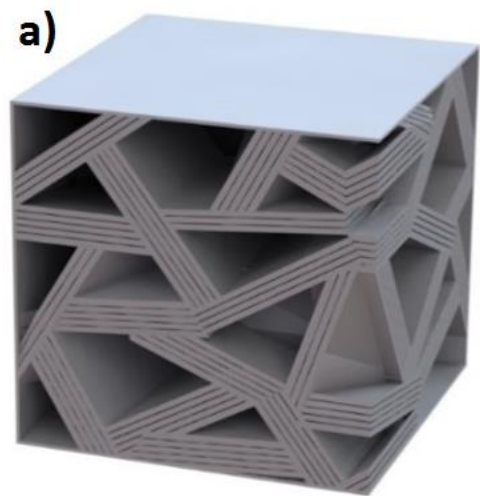

$\mathrm{Ca}_{3} \mathrm{Co}_{4} \mathrm{O}_{9}$ b)

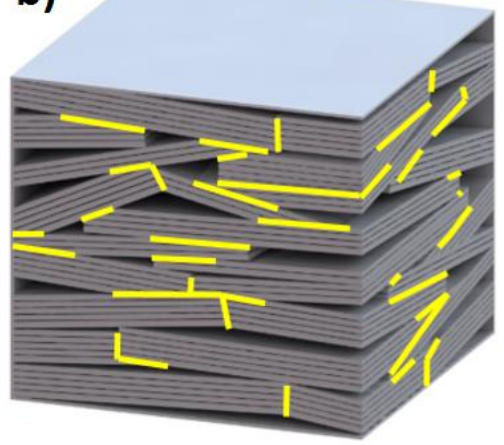

$\mathrm{Ca}_{3} \mathrm{Ba}_{0.05} \mathrm{Co}_{4} \mathrm{O}$
$\mathrm{Ca}_{3} \mathrm{CO}_{4} \mathrm{O}_{9}$ grain interior

Barium at grain boundary

Figure 50 Microstructure drawing of undoped $\mathrm{Ca}_{3} \mathrm{CO}_{4} \mathrm{O}_{9}$ and $\mathrm{Ca}_{3} \mathrm{Ba}_{0.05} \mathrm{Co}_{4} \mathrm{O}_{9}$.

Seeing both bismuth and barium independently achieve impressive thermoelectric results motivates the question of whether both dopants can be used in tandem. The goal would be to implement a dual dopant scenario in order to achieve dual benefits of bismuth improving the crystal texture through grain alignment and grain growth, along with both bismuth and barium segregating at the grain boundary to enhance the Absolute Seebeck coefficient. This can possibly further optimize the thermoelectric performance of the $\mathrm{Ca}_{3} \mathrm{C}_{4} \mathrm{O}_{9}$ and is the motivation for the next three chapters. 


\subsection{References}

1 Gagnon, Steve. It's Elemental: The Periodic Table of Elements. The Element Barium. Jefferson Lab. 2017

2 Winter, Mark. The University of Sheffield and WebElements Ltd, UK. 2017.

${ }^{3}$ Sajid Butt, Yaoyu Ren, Muhammad Umer Farooq, Bin Zhan, Rizwan Ur Rahman Sagar, Yuanhua Lin, Ce-Wen Nan. Enhanced thermoelectric performance of heavy-metals (M: $\mathrm{Ba}, \mathrm{Pb})$ doped misfit-layered ceramics: $\left(\mathrm{Ca}_{2}-\mathrm{M}_{\mathrm{x}} \mathrm{CoO}_{3}\right)_{0.62}\left(\mathrm{CoO}_{2}\right)$. Energy Conversion and Management Vol.83, pp.35-41, 2014.

4 Zhang Feipeng, Lu Qingmei, Li Tingzian, Zhang Xin, Zhang Jiuxing, Song Xiaoyan. Preparation and thermoelectric transport properties of $\mathrm{Ba}$-, La- and Ag-doped $\mathrm{Ca}_{3} \mathrm{Co}_{4} \mathrm{O}_{9}$ oxide materials. Journal of Rare Earths, Vol.3 No.8, pp.778-783, 2013.

5 G. Constantinescu, Sh. Rasekh, M. A. Torres, M. A. Madre, A. Sotelo, J. C. Diez. Improvement of thermoelectric properties in $\mathrm{Ca}_{3} \mathrm{CO}_{4} \mathrm{O}_{9}$ ceramics by $\mathrm{Ba}$ doping. Journal of Materials Science: Materials Electron. Vol.26, pp. 3466-3473, 2015.

6 Marat Gunes, Macit Ozenbas. Effect of grain size and porosity on phonon scattering enhancement of $\mathrm{Ca}_{3} \mathrm{Co}_{4} \mathrm{O}_{9}$. Journal of Alloys and Compounds Vol.626, pp.360-367, 2015.

7 Boyle, Cullen; Calvillo, Paulo; Chen, Yun; Barbero, Ever J.; Mclntyre, Dustin. Grain boundary segregation and thermoelectric performance enhancement of bismuth doped calcium cobaltite. Journal of the European Ceramic Society 36(3):601-607 Elsevier 2016 0955-2219

8 J. J. Shen, X. X. Liu, T. J. Zhu, X. B. Zhao. Improved thermoelectric properties of Ladoped $\mathrm{Bi}_{2} \mathrm{Sr}_{2} \mathrm{Co}_{2} \mathrm{O}_{9}$ - layered misfit oxides. Journal of Materials Science. Vol.44, pp.1889-1893, 2009.

${ }^{9}$ K. Rubešová, T. Hlásek, V. Jakeš, Š. Huber, J. Hejtmánek, D. Sedmidubský. Effect of a powder compaction process on the thermoelectric properties of $\mathrm{Bi}_{2} \mathrm{Sr}_{2} \mathrm{Co}_{1.8} \mathrm{O}_{x}$ ceramics. Journal of the European Ceramic Society, Vol.35, pp.525-531, 2015.

10 Calvillo, Paulo. Chen, Yun. Boyle, Cullen. Barnes, Paul. N. Song, Xueyan. Thermoelectric performance enhancement of calcium cobaltite through barium grain boundary segregation. Inorganic Chemistry. American Chemical Society Publications. September 11, 2015. 


\section{Chapter 7:}

\section{Non-Stoichiometric dual doping of Bismuth and Barium}

\subsection{Motivation of introducing dual dopants using non-stoichiometric addition}

This has been the first approach of dual doping to tune the lattice of the $\mathrm{Ca}_{3} \mathrm{C}_{4} \mathrm{O}_{9}$ and the grain boundary simultaneously. It is also the first approach of dual doping using the non-stoichiometric addition approach. After studying the effects of the nonstoichiometric addition of barium it was found that the dopant deposits itself at the grain boundary enhancing the Absolute Seebeck Coefficient. That coupled with the fact that bismuth deposits itself into the lattice to effect the grain growth and at the grain boundary to enhance the Absolute Seebeck coefficient allows for the possible simultaneous enhancement of both the electrical resistivity and the Absolute Seebeck Coefficient beyond what the other chemistries were capable of individually.

The next three chapters discuss the dual doping of two elements into the $\mathrm{Ca}_{3} \mathrm{Co}_{4} \mathrm{O}_{9}$ baseline. Two methods were investigated in order to compare the effects of cation substitution versus non-stoichiometric addition. The two chemicals being used to dope the $\mathrm{Ca}_{3} \mathrm{Co}_{4} \mathrm{O}_{9}$ were bismuth and barium. The non-stoichiometric addition of barium as a doping agent was studied and analyzed in order to determine its efficiency. Barium was found to enhance the Absolute Seebeck coefficient greatly at room temperature along with maintaining a low electrical resistivity throughout all temperature ranges due to its semi-conductive nature. Its Absolute Seebeck coefficient at low temperatures exceeded 
that of Bismuth's with Bismuth yielding a lower electrical resistivity. From this the two dopings were used to analyze the efficiency of the bismuth and barium working in tandem.

This coupling allows the Bismuth to be utilized at the grain to trigger its growth and alignment in order to obtain a low electrical resistivity while benefiting from having both the bismuth and barium deposit themselves at the grain boundary in order to increase the Absolute Seebeck coefficient. Although barium has a higher Absolute Seebeck coefficient than bismuth at room temperature, bismuth's Absolute Seebeck coefficient rises more sharply causing them to be comparable at high temperatures. Barium's semi-conductive nature was also utilized to help maintain bismuth's already low resistivity at room temperature.

Chapter seven explores the dual doping of bismuth non-stoichiometric addition and barium non-stoichiometric addition in tandem. Individually both were the optimal methods for their respective sets. This set was not only compared with the $\mathrm{Ca}_{3} \mathrm{CO}_{4} \mathrm{O}_{9}$ baseline but also to the non-stoichiometric addition method of bismuth doped alone. This was the compare their dual nature directly to a set with similar chemistry. The addition of bismuth 0.3 was the optimal concentration; however, since the sample contains no vacancies it has the potential to be over doped much quicker, so bismuth 0.2 was used as the starting concentration.

\subsection{Experimental Procedure}

$\mathrm{Ca}_{3} \mathrm{Bi}_{4} \mathrm{Ba}_{w} \mathrm{Co}_{4} \mathrm{O}_{9}$ powders, where $\mathrm{u}=0.0 \& 0.2$ and $\mathrm{w}=0.02,0.05,0.07$ were obtained through a sol-gel route in which non-stoichiometric ratios of $\mathrm{Ca}\left(\mathrm{NO}_{3}\right)_{2} \bullet 4 \mathrm{H}_{2} \mathrm{O}$, $\mathrm{Co}\left(\mathrm{NO}_{3}\right)_{2} \cdot 6 \mathrm{H}_{2} \mathrm{O}, \mathrm{Bi}\left(\mathrm{NO}_{3}\right)_{2} \cdot 5 \mathrm{H}_{2} \mathrm{O}$ and $\mathrm{Ba}\left(\mathrm{NO}_{3}\right)_{2}$ were mixed in deionized water. Ethylene 
glycol and polyethylene glycol were used to aid polymerization of the solution and to aid in particle size. ${ }^{[1]}$ Lastly, nitric acid was added to induce nitrate salts decomposition and facilitate new compound formation. ${ }^{[2]}$

The liquid solution was then submerged in a silicone oil bath on a hot plate and mechanically stirred at $353 \mathrm{~K}$ for 3 hours to achieve the sol-gel state. The sol-gel was then distributed to ceramic crucibles loosely covered with aluminum foil and put into a box furnace with a ramp rate of $10 \mathrm{~K}$ per minute and held at $773 \mathrm{~K}$ for 2 hours then cooled at $10 \mathrm{~K}$ per minute. Holding the temperature here, at $773 \mathrm{~K}$, for the allotted time will ensure that any inorganic material is removed from the material. The ashes were then suspended in ethanol and ball milled for 20 minutes alternating between 1 minute of milling and 1 minute of resting, in order to aid in the dissipation of heat. The powders were deposited in a tubular furnace where they were calcined in an oxygen rich induced environment with a ramp rate of $10 \mathrm{~K}$ per minute and held at $973 \mathrm{~K}$ for 4 hours, to achieve the $\mathrm{Ca}_{3} \mathrm{Co}_{4} \mathrm{O}_{9}$ crystal lattice. The powders were then cooled down to room temperature at a rate of 10 K per minute.

Two pellets were pressed, one to test its electrical properties and the other for its thermal properties. The calcined powders were pressed uniaxially with $1 \mathrm{GPa}$ of force and held for 10 minutes at $298 \mathrm{~K}$. The pellets' densities were obtained before they were put in to a tubular furnace to be sintered. The sintering process removes any extra moisture the pellet may contain and assures proper grain alignment. Pellets were sintered in an oxygen rich induced environment with a ramp rate of $10 \mathrm{~K}$ per minute and held at $1233 \mathrm{~K}$ for 9 hours then cooled at a rate of $4 \mathrm{~K}$ per minute and once again the densities were taken. Pellets were cut into a $2 \mathrm{~mm} \times 3.5 \mathrm{~mm} \times 9 \mathrm{~mm}$ rectangular shapes and 
inserted into a Linseis LSR 3-Seebeck machine, subjected to a low pressure helium, He, environment where the pellet's electrical resistivity and Absolute Seebeck Coefficient were simultaneously determined by means of a dc four-probe method. Since helium is an inert gas it will not react or interfere with the measurement. A second pellet was cut along a different axis and inserted into the Linseis LFA 1000 machine to test its thermal conductivity. The pellet was cut to be $2 \mathrm{~mm}$ thick and polished to a diameter of $11-13 \mathrm{~mm}$. Any less than $11 \mathrm{~mm}$ and there would be room for heat to escape the holder, any more than $13 \mathrm{~mm}$ and the pellet would be too large for the holder.. The thermal conductivity is calculated by the equation

$$
K=\lambda C_{p} \rho_{m}
$$

Where $\lambda$ is the thermal diffusity in meters squared per seconds $\left(\mathrm{m}^{2} / \mathrm{s}\right), \mathrm{C}_{\mathrm{p}}$ is the specific heat capacity in joules per kilogram kelvin $(\mathrm{J} / \mathrm{kg} \cdot \mathrm{K})$, and $\rho_{\mathrm{m}}$ is the mass density in grams per centimeter cubed $\left(\mathrm{g} / \mathrm{cm}^{3}\right)$. As stated in the first section, these are the three values needed in order to obtain the materials' ZT value. Bismuth non-stoichiometric addition has been reported on and a concentration of 0.2 was used and held constant throughout the varied barium dopings. A non-stoichiometric addition method was used for varying the doping concentrations of barium. Their chemistries are as follows: $\mathrm{Ca}_{3} \mathrm{Bi}_{u} \mathrm{Ba}_{w} \mathrm{C}_{4} \mathrm{O}_{9}$ where $u=0.0,0.2$ and $w=0.0,0.002,0.02,0.05$, and 0.07 .

\subsection{Finding a balance between carrier mobility \& concentration through tuning dopant concentration}

Figure 51 shows that at low temperatures the electrical resistivities of the bismuth barium dual doped samples were higher than that of bismuth 0.2 non-stoichiometric 
sample alone. As the temperature increased this gap closed drastically with the $\mathrm{Ca}_{3} \mathrm{Bi}_{0.2} \mathrm{Ba}_{0.02} \mathrm{Co}_{4} \mathrm{O}_{9}$ dual doped chemistry coming in just less than $10 \mu \Omega m$ higher than the $\mathrm{Ca}_{3} \mathrm{Bi}_{0.2} \mathrm{Co}_{4} \mathrm{O}_{9}$ nonstoichiometric chemistry at $1080 \mathrm{~K}$. This is largely due to the semiconductor characteristics that barium contributes to this chemistry. ${ }^{[3]}$ As bismuth acts like a metal and its electrical resistivity increases over temperature, the barium concentration in the dual doped sample contributes to act more like a semi-conductor decreasing the electrical resistivity with increasing temperature, allowing the gap to close. ${ }^{[3,4]}$ The $\mathrm{Ca}_{3} \mathrm{Bi}_{0.2} \mathrm{Co}_{4} \mathrm{O}_{9}$ non-stoichiometric chemistry shown in figure 51 is $\sim 5 \mu \Omega \mathrm{m}$ lower than its $\mathrm{Ca}_{2.8} \mathrm{Bi}_{0.2} \mathrm{CO}_{4} \mathrm{O}_{9}$ cation substitution counterpart from chapter 4 across the whole temperature spectrum. However, the electrical resistivities of the dual doped samples were over 5 times larger than the $\mathrm{Ca}_{3} \mathrm{Bi}_{0.2} \mathrm{C}_{4} \mathrm{O}_{9}$ non-stoichiometric chemistry. 


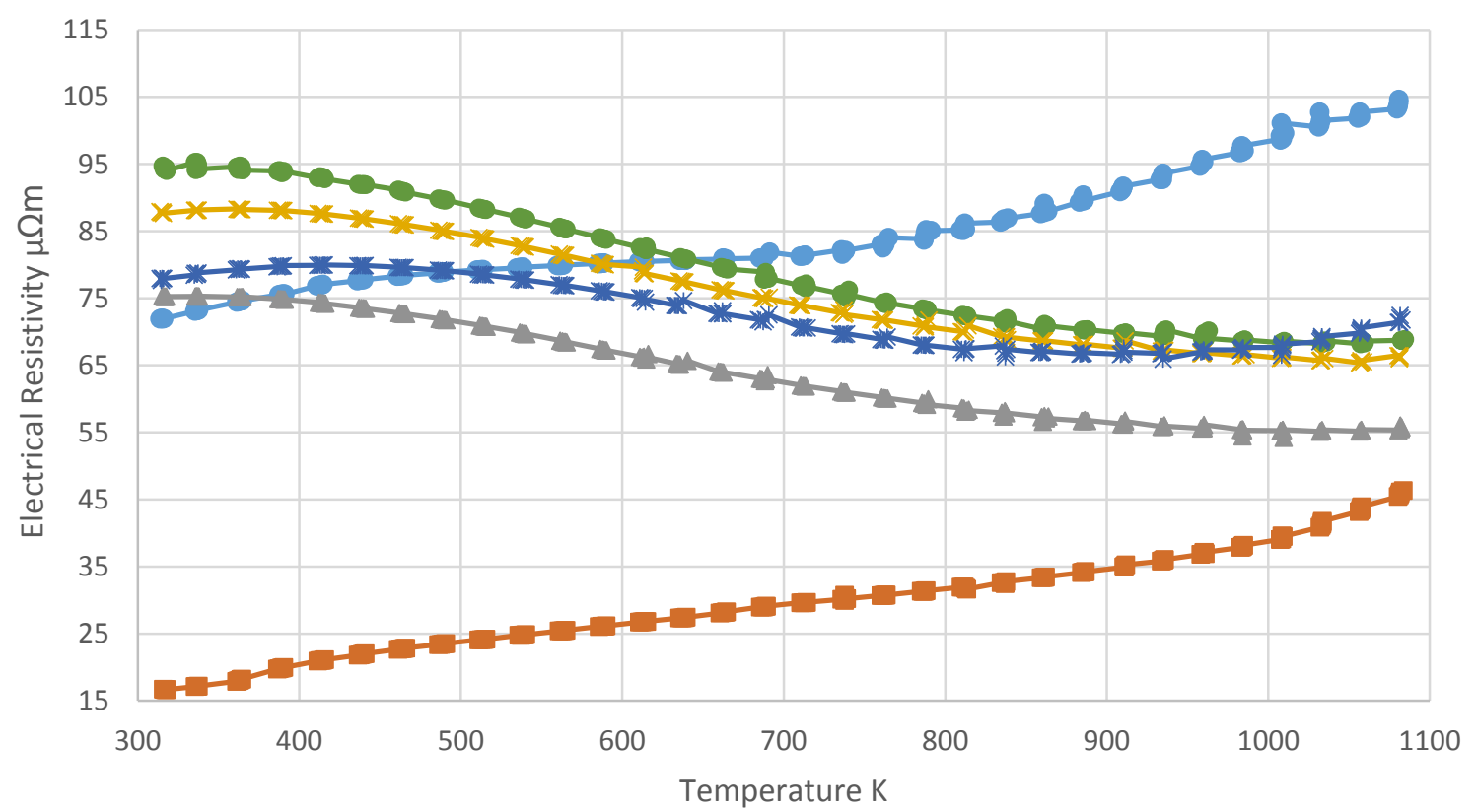

$-\mathrm{Ca3C0409}$

Figure 51 Temperature dependence of electrical resistivity for $\mathrm{Ca}_{3} \mathrm{Bi}_{4} \mathrm{Ba}_{w} \mathrm{Co}_{4} \mathrm{O}_{9}$ bismuth $\&$ barium non-stoichiometric addition where $u=0.0,0.02,0.05$, and 0.07 .

Such an increase in electrical resistivity has to do with the over saturation of dopants in the sample. The addition of both dopants simultaneously proved to over dope the sample at low concentrations considering both dopants are predominantly depositing themselves at the grain boundary. This most likely created a situation indicative of an over doping at a much lower concentration than expected. Due to the reduction in electrical resistivity there is either a drastic deficiency in carrier mobility, carrier concentration or both. ${ }^{[5,6,7,8,9,10]}$ Another probability is the formation of a secondary phase at the grain boundary. 
Having both bismuth and barium deposit themselves at the grain boundary simultaneously has proven to enhance the Absolute Seebeck coefficient beyond the capabilities of bismuth non-stoichiometric addition alone as is evident from figure 52 . Barium's semi-conductor characteristics allow the electrical resistivity to maintain a low value all the way up to the high temperatures but the Absolute Seebeck coefficient now suffers in this same temperature range. This decrease reiterates an increase in carrier concentration at the grain boundary causing the reduced Absolute Seebeck Coefficient. $[5,6,11,14,12]$

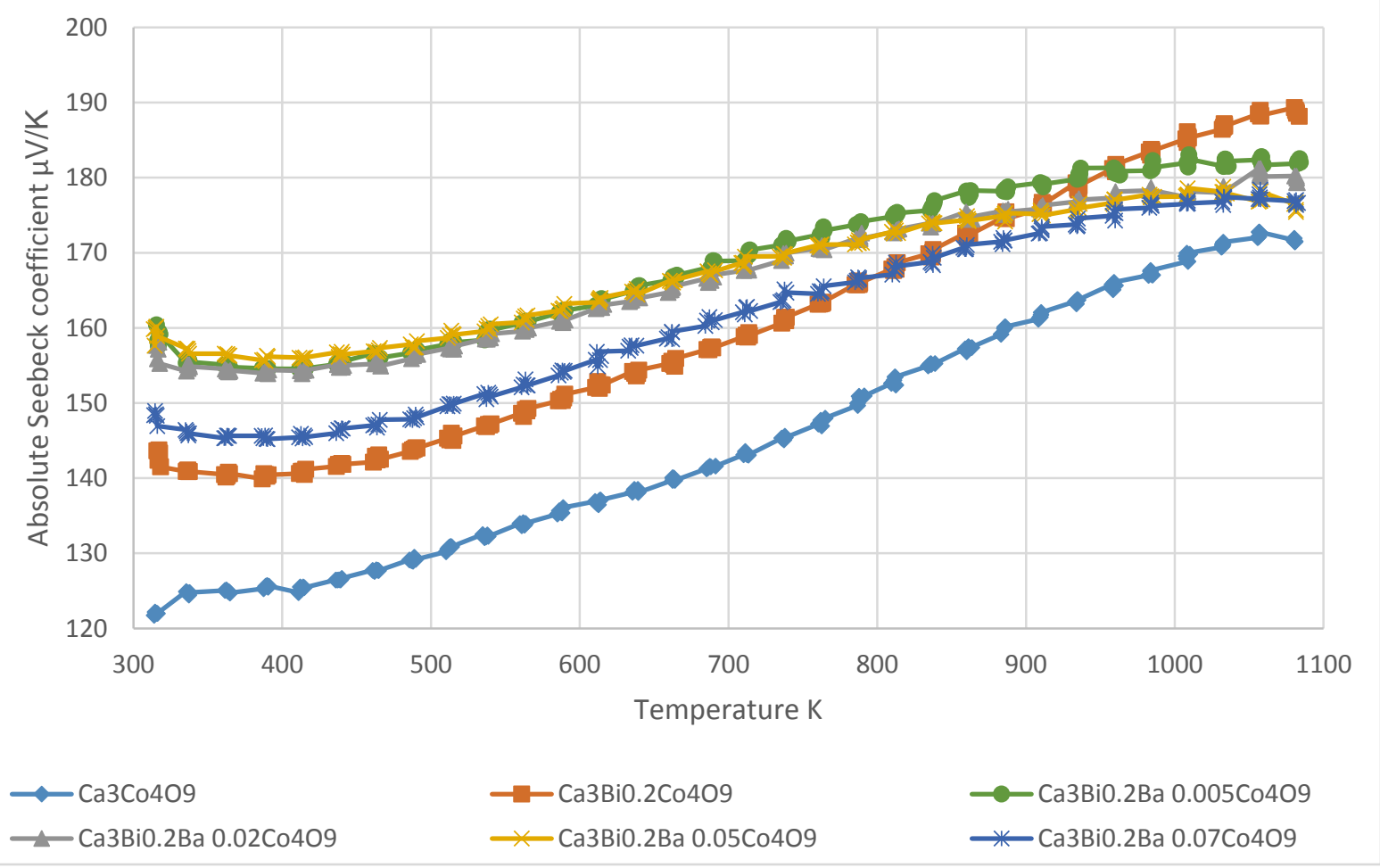

Figure 52 Temperature dependence of absolute seebeck coefficient for $\mathrm{Ca}_{3} \mathrm{Bi}_{4} \mathrm{Ba}_{w} \mathrm{Co}_{4} \mathrm{O}_{9}$ bismuth \& barium non-stoichiometric addition where $u=0.0,0.02,0.05$, and 0.07. 
For the electrical resistivity to decrease at high temperatures the way it demonstrates here, there must have been an exceptional carrier mobility increase in order to offset the carrier concentration decrease. ${ }^{[5,6,7,10]}$ Having both the bismuth and barium deposit themselves at the grain boundary proved to decrease the carrier concentration. $[5,6,12]$ This is normally responsible for increasing the Absolute Seebeck coefficient but was inferior compared to the drastic decrease in mobility causing a slower increase in the Absolute Seebeck coefficient. This shows that one of the dopants were forced into the lattice. In this case any competition at the grain boundary leads to barium depositing at the boundary, due to its ionic size and inability to insert itself into the lattice. This leaving bismuth to go to the grain interior over doping the lattice which is demonstrated through their Absolute Seebeck coefficients in figure 52. ${ }^{[12,13,14]}$

High electrical resistivity could not offset the enhanced Absolute Seebeck coefficient which then eventually lead to a power factor that was lower in the dual dopant chemistries in comparison with the non-stoichiometric addition of bismuth. This can be seen in figure 53 where the dual doped samples are only around $25 \%$ of the bismuth doped sample at low temperatures. 


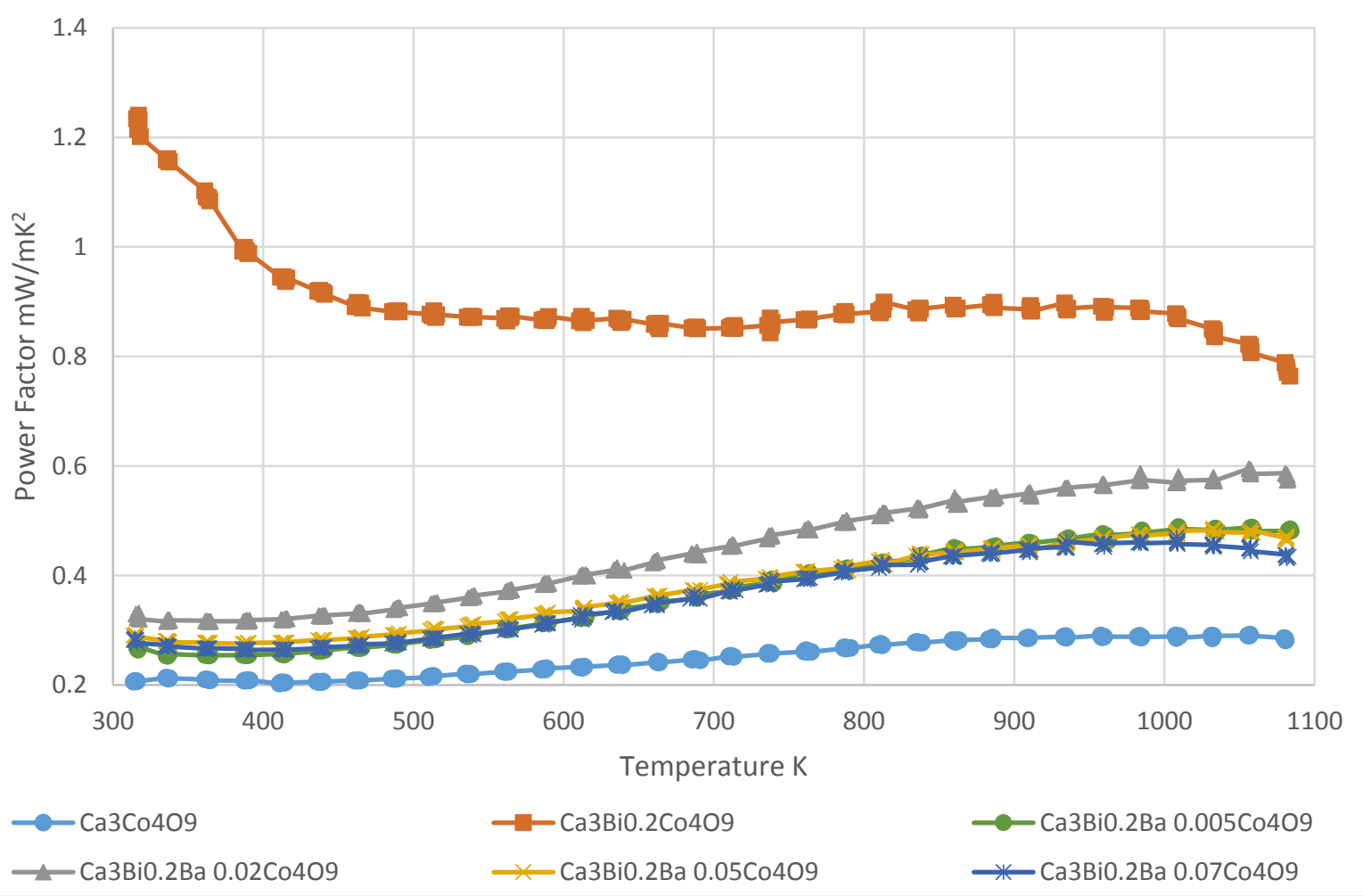

Figure 53 Temperature dependence of power factor for $\mathrm{Ca}_{3} \mathrm{Bi}_{4} \mathrm{Ba}_{w} \mathrm{CO}_{4} \mathrm{O}_{9}$ bismuth \& barium non-stoichiometric addition where $u=0.0,0.02,0.05$, and 0.07 .

\subsection{Summary of results for doping $\mathrm{Ca}_{3} \mathrm{Co}_{4} \mathrm{O}_{9}$ using dual dopants Bismuth \& Barium through non-stoichiometric addition}

The dual doping of bismuth and barium through non-stoichiometric addition to the $\mathrm{Ca}_{3} \mathrm{C}_{4} \mathrm{O}_{9}$ oxide was successful in enhancing the room temperature Absolute Seebeck Coefficient to $\sim 160 \mu \mathrm{V} / \mathrm{K}$. However, due to over doping, the electrical resistivity suffered and yielded a much greater value across all temperatures than it did for the optimal samples of both the bismuth and barium non-stoichiometric addition methods individually. As seen in figure 53 the $\mathrm{Ca}_{3} \mathrm{Bi}_{0.2} \mathrm{C}_{04} \mathrm{O}_{9}$ sample had a power factor of $1.23 \mathrm{~mW} / \mathrm{mK}^{2}$ at $315 \mathrm{~K}$ which is about 4 times greater than the $0.33 \mathrm{~mW} / \mathrm{mK}^{2}$ power factor that the optimal 
dual doped sample achieved at the same temperature. At $982 \mathrm{~K}$ the $\mathrm{Ca}_{3} \mathrm{Bi}_{0.2} \mathrm{Co}_{4} \mathrm{O}_{9}$ sample achieves a power factor of $0.89 \mathrm{~mW} / \mathrm{mK}^{2}$ which is only about $50 \%$ higher than the $0.57 \mathrm{~mW} / \mathrm{mK}^{2}$ power factor the dual doped sample achieved at the same temperature. Despite having a higher electrical resistivity, the presence of the barium was able to maintain its value across all temperatures which explains why the power factor was $25 \%$ that of the optimal chemistry used in the bismuth non-stoichiometric addition set at low temperatures and about $65 \%$ of the optimal chemistry at high temperatures. For this reason more chemistries and concentrations of this set were tested to find the proper doping levels in order to achieve the perfect balance in carrier mobility and carrier concentration to enhance the Absolute Seebeck coefficient while achieving and maintaining a low electrical resistivity. 


\subsection{References}

1 Marat Gunes, Macit Ozenbas. Effect of grain size and porosity on phonon scattering enhancement of $\mathrm{Ca}_{3} \mathrm{C}_{4} \mathrm{O}_{9}$. Journal of Alloys and Compounds Vol.626, pp.360-367, 2015.

2 Boyle, Cullen; Calvillo, Paulo; Chen, Yun; Barbero, Ever J.; Mclntyre, Dustin. Grain boundary segregation and thermoelectric performance enhancement of bismuth doped calcium cobaltite. Journal of the European Ceramic Society 36(3):601-607 Elsevier 2016 0955-2219

3 J. J. Shen, X. X. Liu, T. J. Zhu, X. B. Zhao. Improved thermoelectric properties of Ladoped $\mathrm{Bi}_{2} \mathrm{Sr}_{2} \mathrm{Co}_{2} \mathrm{O}_{9}$ - layered misfit oxides. Journal of Materials Science. Vol.44, pp.1889-1893, 2009.

${ }^{4}$ Live Science Staff. Facts about Bismuth. September 5, 2013.

5 Tao Wu, Trevor A. Tyson, Haiyan Chen, Jianming Bai, Hsin Wang and Cherno Jaye. A structural phase transition in $\mathrm{Ca}_{3} \mathrm{Co}_{4} \mathrm{O}_{9}$ Associated with enhanced high temperature thermoelectric properties. Cornell University Library. April 15, 2012.

6 T. Takeuchi, T. Kondo, K. Soda, U. Mizutani, R. Funahashi, M. Shikano, S. Tsuda, T. Yokoya, S. Shin, T. Muro. Electronic structure and large thermoelectric power in $\mathrm{Ca}_{3} \mathrm{C}_{4} \mathrm{O}_{9}$. Journal of Electron Spectroscopy and Related Phenomena 137-140 2004 595-599. March 18, 2004.

7 Boyle, Cullen; Calvillo, Paulo; Chen, Yun; Barbero, Ever J.; Mclntyre, Dustin. Grain boundary segregation and thermoelectric performance enhancement of bismuth doped calcium cobaltite. Journal of the European Ceramic Society 36(3):601-607 Elsevier 2016 0955-2219

8 Masashi Mikami, Kanju Chong, Yuzuru Miyazaki, Tsuyoshi Kajitani, Takahiro Inoue, Satoshi Sodeoka, and Ryoji Funahashi. Bi-Substitution effects on crystal structure and thermoelectric properties of $\mathrm{Ca}_{3} \mathrm{C}_{4} \mathrm{O}_{9}$ single crystals. Japanese Journal of Applied Physics. Vol.45, No.5A, pp.4131-4136, 2006.

9 Sajid Butt, Yaoyu Ren, Muhammad Umer Farooq, Bin Zhan, Rizwan ur Rahman Sagar, Yuanhua Lin, Ce-Web Nan. Enhanced thermoelectric performance of heavy-metals (M: $\mathrm{Ba}, \mathrm{Pb})$ doped misfit-layered ceramics: $\left(\mathrm{Ca}_{2}-\mathrm{x} \mathrm{M}_{\mathrm{x}} \mathrm{CoO}_{3}\right)_{0.62}\left(\mathrm{CoO}_{2}\right)$. Energy Conversion and Management. Vol.83, pp.35-41, 2014.

10 T. Sun, H. H. Hng, Q. Y. Yan, and J. Ma. Enhanced high temperature thermoelectric properties of $\mathrm{Bi}$-doped $\mathrm{c}$-axis oriented $\mathrm{Ca}_{3} \mathrm{C}_{4} \mathrm{O}_{9}$ thin films by pulsed laser deposition. Journal of Applied Physics. Vol.108, 2010.

11 Calvillo, Paulo. Chen, Yun. Boyle, Cullen. Barnes, Paul. N. Song, Xueyan. Thermoelectric performance enhancement of calcium cobaltite through barium grain boundary segregation. Inorganic Chemistry. American Chemical Society Publications. September 11, 2015. 
${ }^{12}$ Guangkun Ren, Jinle Lan, Chengcheng Zeng, Yaochun Liu, Bin Zhan, Sajid Butt, YuanHua Lin, and Ce-Wen Nan. High performance oxides-based thermoelectric materials. Journal of Minerals, Metals \& Materials Society. Vol.67, No.1, pp.211-221, 2015.

${ }^{13}$ CrystalMaker Software Ltd. 2017.

${ }^{14}$ Masashi Mikami, Kanju Chong, Yuzuru Miyazaki, Tsuyoshi Kajitani, Takahiro Inoue, Satoshi Sodeoka, and Ryoji Funahashi. Bi-Substitution effects on crystal structure and thermoelectric properties of $\mathrm{Ca}_{3} \mathrm{CO}_{4} \mathrm{O}_{9}$ single crystals. Japanese Journal of Applied Physics. Vol.45, No.5A, pp.4131-4136, 2006.

15 C. S. Huang, F. P. Zhang, X. Zhang, Q.M. Lu, J. X. Zhang, Z. Y. Liu. Enhanced thermoelectric figure of merit through electrical and thermal transport modulation by dual-doping and texture modulating for $\mathrm{Ca}_{3} \mathrm{C}_{4} \mathrm{O}_{9+\delta}$ oxide materials. Journal of Alloys and Compounds. Vol.687, pp.87-94, 2016.

16 Ruoming Tian, Tianshu Zhang, Dewei Chu, Richard Donelson, Li Tao, Sean Li. Enhancement of high temperature thermoelectric performance in $\mathrm{Bi}$, Fe co-doped layered oxide-based material $\mathrm{Ca}_{3} \mathrm{C}_{4} \mathrm{O}_{9+\delta}$. Journal of Alloys and Compounds. Vol.615, pp.311-315, 2014.

17 Q. M. Lu, J. X. Zhang, Q. Y. Zhang, Y. Q. Liu, and D. M. Liu. Improved thermoelectric properties of $\mathrm{Ca}_{3-x} \mathrm{Ba}_{x} \mathrm{CO}_{4} \mathrm{O}_{9}(\mathrm{x}=0 \sim 0.4)$ bulks by sol-gel and SPS method. $15^{\text {th }}$ International Conference of Thermoelectrics. 2006.

18 G. Constantinescu, Sh. Rasekh, M. A. Torres, M. A. Madre, A. Sotelo, J. C. Diez. Improvement of thermoelectric properties in $\mathrm{Ca}_{3} \mathrm{CO}_{4} \mathrm{O}_{9}$ ceramics by $\mathrm{Ba}$ doping. Journal of Materials Science., Vol.26, pp.3466-3473, 2015.

18 Haoshan Hao, Limin Zhao, and Xing Hu. Microstructure and thermoelectric properties of Bi-and $\mathrm{Cu}$-substituted $\mathrm{Ca}_{3} \mathrm{Co}_{4} \mathrm{O}_{9}$ oxides. Journal of Materials Science technology, Vol.25, pp.105-108, 2009.

19 Zunyi Tian, Xiaohong Wang, Jun Liu, Zhongjin Lin, Yangsen Hu, Yigui Wu, Chao Han, Zhiyu $\mathrm{Hu}$. Power factor enhancement induced by $\mathrm{Bi}$ and $\mathrm{Mn}$ co-substitution in $\mathrm{Na}_{\times} \mathrm{CoO}_{2}$ thermoelectric materials. Journal of Alloys and Compounds. Vol.661, pp.161-167, 2016.

20 Zhang Feipeng, Lu Qingmei, Li Tingxian, Zhang Xin, Zhang Jiuxing, Song Xiaoyan. Preparation and thermoelectric transport properties of $\mathrm{Ba}-$, La-, and Ag- doped $\mathrm{Ca}_{3} \mathrm{Co}_{4} \mathrm{O}_{9}$ oxide materials. Journal of Rare Earths. Vol.31, No.8, pp.778-783, 2013.

21 Siwen Li, Ryoji Funahashi, Ichiro Matsubara, Kazuo Ueno, Satoshi Sodeoka, and Hiroyuki Yamada. Synthesis and thermoelectric properties of the new oxide materials $\mathrm{Ca}_{-x} \mathrm{Bi}_{\times} \mathrm{C}_{4} \mathrm{O}_{9+\delta}(0.0<\mathrm{x}<0.75)$. Chemical Material, Vol.12, pp.2424-2427, 2000.

22 Haoshan Hao, Qinglin He, ChangQing Chen, Hongwei Sun, and Xing Hu. Textured structure and anisotropic thermoelectric properties of $\mathrm{Ca}_{2.7} \mathrm{Bi}_{0.3} \mathrm{C}_{0} \mathrm{O}_{9}$ oxide prepared by conventional solid-state reaction. International Journal of Modern Physics B. Vol.23, No.1, pp.87-95, 2009.

${ }^{23}$ Ankam Bhaskar, Z. -R. Lin, Chia-Jyi Liu. Thermoelectric properties of $\mathrm{Ca}_{2.95} \mathrm{Bi}_{0.05} \mathrm{Co}_{4}-$ хFexO $\mathrm{O}_{9+\delta}(0<\mathrm{x}<0.15)$. Energy Conversion and Management. Vol.76, pp.63-67, 2013. 


\section{Chapter 8: \\ Dual Doping $\mathrm{Ca}_{3} \mathrm{Co}_{4} \mathrm{O}_{9}$ through Cationic Substitution \& Non-Stoichiometric Addition}

\subsection{Background and motivation for dual doping with cation substitution and non- stoichiometric addition}

Dual doping is not necessarily a new concept and it has been utilized and explored in the last chapter through the non-stoichiometric addition method. However, there are no reportings in the literatures on the dual doping while utilizing the cationic substitution and non-stoichiometric addition in tandem. The last chapter discussed the dual doping of bismuth and barium using the nonstoichiometric addition. This chapter explores the combination of both the cationic substitution and nonstoichiometric addition. The $\mathrm{Ca}_{3} \mathrm{Co}_{4} \mathrm{O}_{9}$ baseline was doped with a set concentration of bismuth by using the cation substitution method where the bismuth atoms replace calcium atoms to improve crystal texture. Barium utilizes the non-stoichiometric addition method to enhance the Absolute Seebeck coefficient and maintain a low electrical resistivity. Since barium can only deposit at the grain boundary it forces the bismuth to mainly deposit at the grain interior. This can cause the sample to be easily over doped, which was seen in the last chapter. By using cation substitution with the non-stoichiometric addition the calcium vacancies give the bismuth a place to deposit lessening the possibility of over doping.

The best concentrations of bismuth cation substitution described in chapter 4 were that of $x=0.2$ and $x=0.3$. Results from the previous chapter proved that this method has a 
great tendency to over dope the sample much easier due to the dual nature. For this reason the lower of the two were used in this chapter as to not over dope the sample. Also this provides a much more linear comparison between this chapter and last considering the same bismuth concentration was used. Barium utilized the method of non-stoichiometric addition once again with the same three concentrations used in chapter 7 , which were barium $0.02,0.05 \& 0.07$, in order to find a trend and see if the Absolute Seebeck Coefficient can be further enhanced thus improving the power factor and overall efficiency.

\subsection{Experimental Procedure}

$\mathrm{Ca}_{3-\mathrm{x}} \mathrm{Bi}_{\times} \mathrm{Ba}_{4} \mathrm{Co}_{4} \mathrm{O}_{9}$ powders, where $\mathrm{x}=0.0 \& 0.2$ and $\mathrm{u}=0.0,0.02,0.05,0.07$ were obtained through a sol-gel route in which stoichiometric ratios of $\mathrm{Ca}\left(\mathrm{NO}_{3}\right)_{2} \bullet 4 \mathrm{H}_{2} \mathrm{O}$, $\mathrm{Co}\left(\mathrm{NO}_{3}\right)_{2} \cdot 6 \mathrm{H}_{2} \mathrm{O}$ and $\mathrm{Bi}\left(\mathrm{NO}_{3}\right)_{2} \cdot 5 \mathrm{H}_{2} \mathrm{O}$ were mixed with non-stoichiometric ratios of $\mathrm{Ba}\left(\mathrm{NO}_{3}\right)_{2}$ in deionized water. Ethylene glycol and polyethylene glycol were used to aid polymerization of the solution and to aid in particle size. ${ }^{[1]}$ Lastly, nitric acid was added to induce nitrate salts decomposition and facilitate new compound formation. ${ }^{[2]}$

The liquid solution was then submerged in a silicone oil bath on a hot plate and mechanically stirred at $353 \mathrm{~K}$ for 3 hours to achieve the sol-gel state. The sol-gel was then distributed to ceramic crucibles loosely covered with aluminum foil and put into a box furnace with a ramp rate of $10 \mathrm{~K}$ per minute and held at $773 \mathrm{~K}$ for 2 hours then cooled at a rate of $10 \mathrm{~K}$ per minute. Holding the temperature here, at $773 \mathrm{~K}$, for the allotted time will ensure that any inorganic material is removed from the material. The ashes were then suspended in ethanol and ball milled for 20 minutes alternating between 1 minute of 
milling and 1 minute of resting in order to aid in the dissipation of heat. The powders were deposited in a tubular furnace where they were calcined in an oxygen rich induced environment with a ramp rate of $10 \mathrm{~K}$ per minute and held at $973 \mathrm{~K}$ for 4 hours, to achieve the $\mathrm{Ca}_{3} \mathrm{C}_{4} \mathrm{O}_{9}$ crystal lattice. The powders were then cooled down to room temperature at a rate of $10 \mathrm{~K}$ per minute.

Two pellets were pressed, one to test its electrical properties and the other for its thermal properties. The calcined powders were pressed uniaxially with $1 \mathrm{GPa}$ of force and held for 10 minutes at $298 \mathrm{~K}$. The pellets' densities were obtained before they were put in to a tubular furnace to be sintered. The sintering process removes any extra moisture the pellet may contain and assures proper grain alignment. Pellets were sintered in an oxygen rich induced environment with a ramp rate of $10 \mathrm{~K}$ per minute and held at $1233 \mathrm{~K}$ for 9 hours. It was then cooled back to room temperature at a $4 \mathrm{~K}$ per minute and once again the densities were taken. Pellets were cut into a $2 \mathrm{~mm} \times 3.5 \mathrm{~mm} \times 9 \mathrm{~mm}$ rectangular shapes and inserted into a Linseis LSR 3-Seebeck machine, subjected to a low pressure helium, He, environment where the pellet's electrical resistivity and Absolute Seebeck Coefficient were simultaneously determined by means of a dc four-probe method. Since helium is an inert gas it will not react or interfere with the measurement. A second pellet was cut along a different axis and inserted into the Linseis LFA 1000 machine to test its thermal conductivity. The pellet was cut to be $2 \mathrm{~mm}$ thick and polished to a diameter of $11-13 \mathrm{~mm}$. Any less than $11 \mathrm{~mm}$ and there would be room for heat to escape the holder, any more than $13 \mathrm{~mm}$ and the pellet would be too large for the holder. The thermal conductivity is calculated by the equation 


$$
K=\lambda C_{p} \rho m
$$

Where $\lambda$ is the thermal diffusity in meters per second $\left(\mathrm{m}^{2} / \mathrm{s}\right), \mathrm{C}_{\mathrm{p}}$ is the specific heat capacity in joules per kilogram kelvin $(\mathrm{J} / \mathrm{kg} \cdot \mathrm{K})$, and $\rho_{m}$ is the mass density grams per centimeter cubed $\left(\mathrm{g} / \mathrm{cm}^{3}\right)$. As stated in the first section, these are the three values needed in order to obtain the materials' ZT value. Bismuth cation substitution has been reported on and a concentration of 0.2 was used and held constant throughout the varied barium dopings. A non-stoichiometric addition was used for varying the doping concentrations of barium. Their chemistries are as follows: $\mathrm{Ca}_{3}-\mathrm{Bi}_{\times} \mathrm{Ba}_{4} \mathrm{C}_{4} \mathrm{O}_{9}$ where $\mathrm{x}=0.0,0.2$ and $\mathrm{u}=$ $0.0,0.02,0.05$, and 0.07 .

\subsection{Comparison of Non-stoichiometric dual doping to Cation Substitution \& Non- stoichiometric dual doping}

In the previous chapter it was shown how the semi-conductor nature of barium allowed the electrical resistivity of the dual doped sample to be competitive with the optimal bismuth non-stoichiometric addition sample at high temperatures, even though it had a much lower electrical resistivity at lower temperatures. ${ }^{[3]}$ The dual doped samples in this chapter start off at low temperatures with an electrical resistivity relatively higher but much closer to the sample doped with only bismuth using the cationic substitution method alone which is evident from figure 54 . This comparable electrical resistivity at low temperatures allows these samples to achieve a similar electrical resistivity at the midhigh temperature range and even surpass the bismuth sample beyond $900 \mathrm{~K}$, due to the ability of barium to act like a semi-conductor. This unique ability that barium has to maintain a low electrical resistivity across all temperatures allows the addition of barium 
to the bismuth cation substitution set to surpass that of bismuth alone at high temperatures. ${ }^{[3]}$ The bismuth aids in lowering the resistivity sufficiently at room temperature and barium's semi-conductor nature causes it to maintain this low resistivity. $[3,4]$

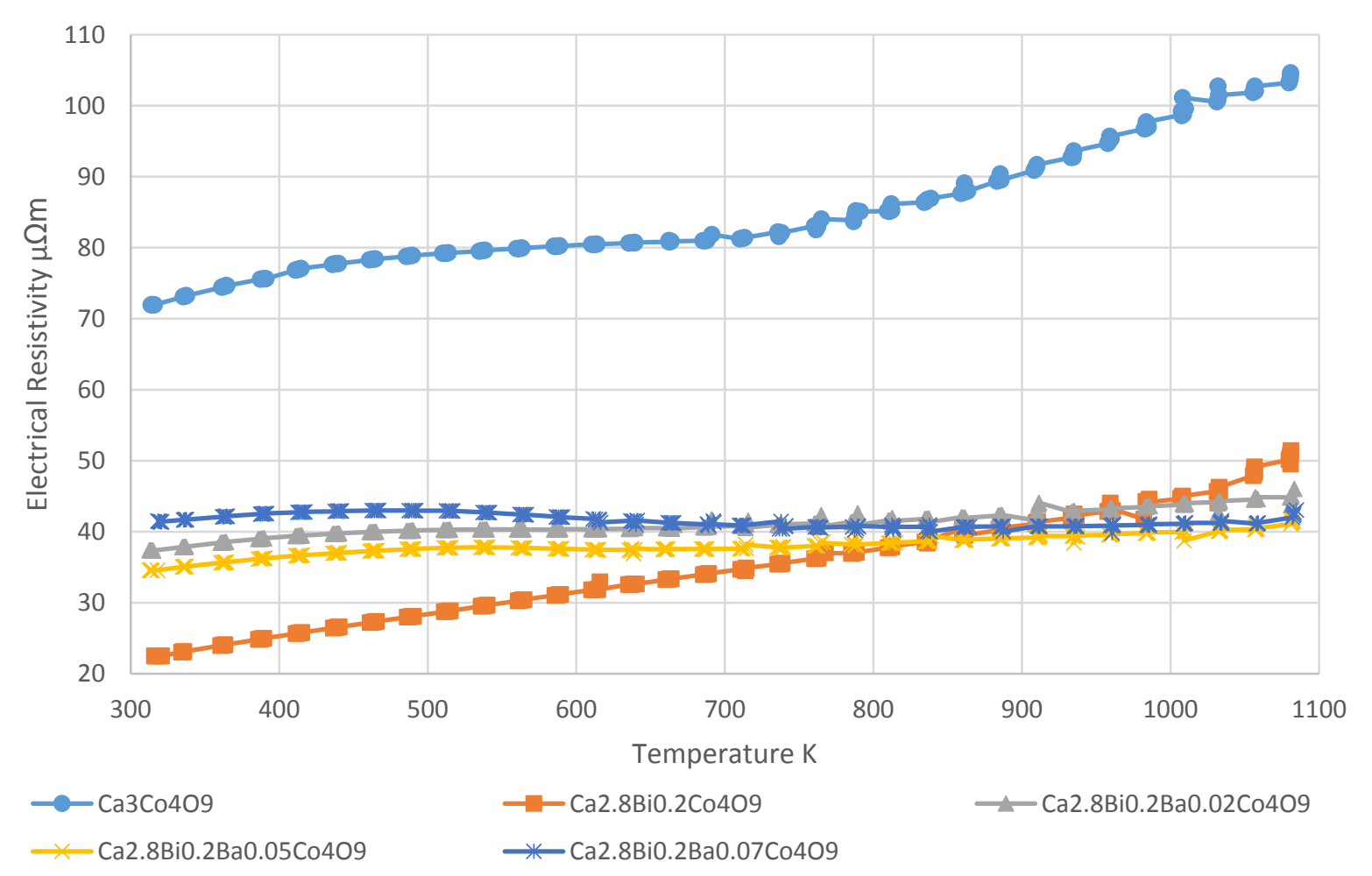

Figure 54 Temperature dependence of electrical resistivity for $\mathrm{Ca}_{3-x} \mathrm{Bi}_{x} \mathrm{Ba}_{4} \mathrm{Co}_{4} \mathrm{O}_{9}$ bismuth cation substitution \& barium non-stoichiometric addition where $u=0.0,0.02$, 0.05 , and 0.07 .

Demonstrated in figure 55, the sample that just includes the cation substitution of bismuth alone experiences an immediate increase in its Absolute Seebeck Coefficient, up to $\sim 145 \mu \mathrm{V} / \mathrm{K}$ in the low temperature regime. This is consistent with the bismuth cation substitution set previously analyzed in chapter 4 . This is due to the bismuth depositing 
itself at the grain boundary and acting as a filter for the carrier concentration. ${ }^{[5,6,7]}$ Once the barium is introduced at the grain boundary this effect is further enhanced achieving a room temperature Absolute Seebeck Coefficient of anywhere between 155-160 $\mu \mathrm{V} / \mathrm{K}$. This shows that barium allows this material to have a much higher saturation level at the grain boundary, predominantly due to the fact barium deposits itself wholly at the grain boundary instead of both the boundary and the lattice as bismuth does. ${ }^{[7,8]}$ The Absolute Seebeck Coefficient increased with increasing temperature at a slow rate compared with the sample of bismuth cation substitution alone. This slow increase that it experiences is caused from the increase in carrier concentration which consequentially caused the reduction in electrical resistivity as the temperature increases. ${ }^{[5,6]}$ 


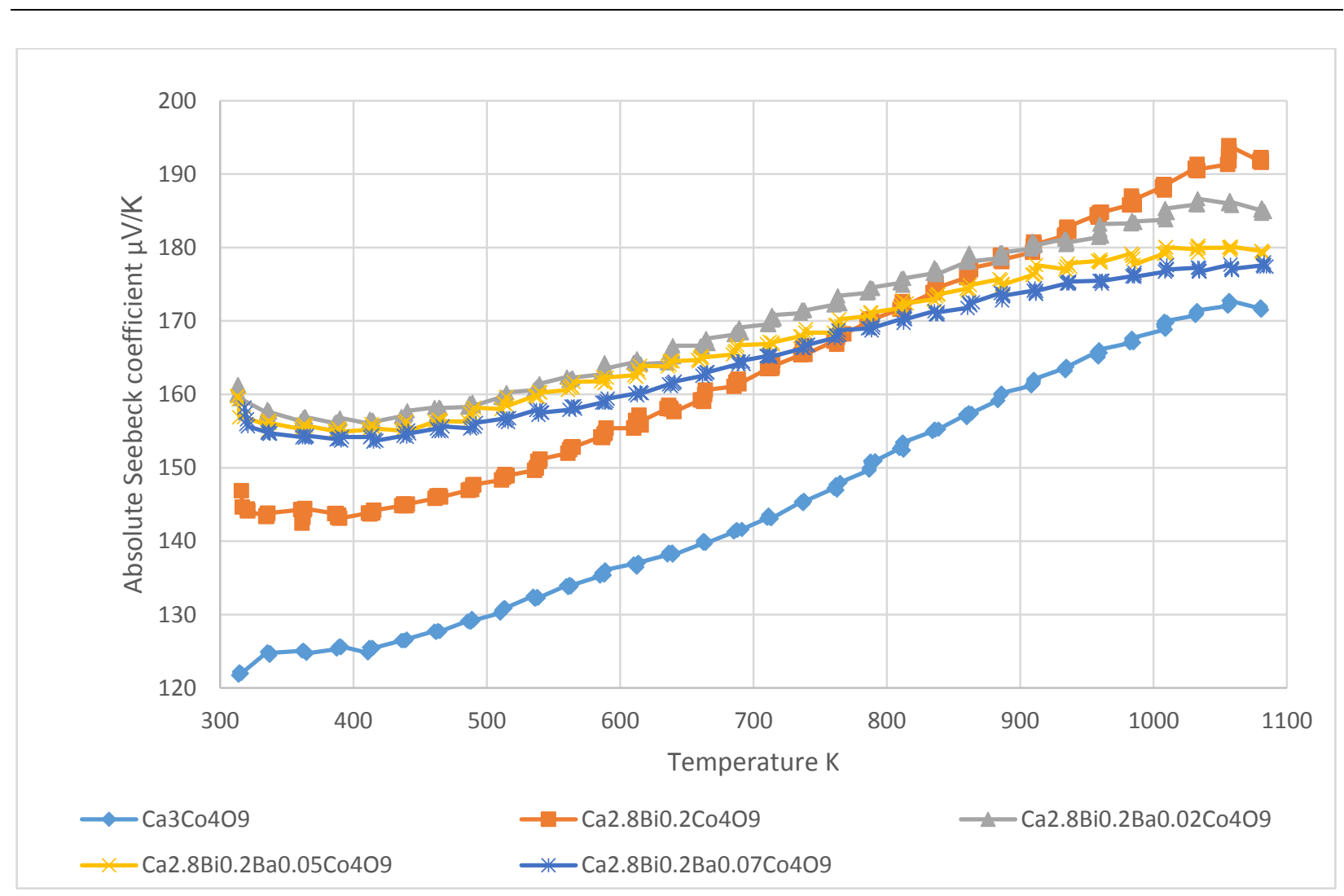

Figure 55 Temperature dependence of absolute seebeck coefficient for $\mathrm{Ca}_{2.8} \mathrm{Bi}_{0.2} \mathrm{Ba}_{4} \mathrm{CO}_{4} \mathrm{O}_{9}$ bismuth cation substitution \& barium non-stoichiometric addition where $u=0.0,0.02,0.05$, and 0.07 .

The main motive behind this sample set was to increase the Absolute Seebeck Coefficient, through grain boundary segregation, while maintaining a low electrical resistivity, through grain texture and alignment. The Absolute Seebeck Coefficient, although increased at low temperature with the presence of barium, did not compare to that of the $\mathrm{Ca}_{2.8} \mathrm{Bi}_{0.2} \mathrm{Co}_{4} \mathrm{O}_{9}$ doping at high temperature. In fact, the semi-conductor nature of the barium providing a low electrical resistivity at high temperatures was responsible for allowing the dual doped set to have a competitive power factor of $0.80 \mathrm{~mW} / \mathrm{mK}^{2}$ at 985 $\mathrm{K}$ with the $\mathrm{Ca}_{2.8} \mathrm{Bi}_{0.2} \mathrm{C}_{4} \mathrm{O}_{9}$, which too had a power factor of $0.80 \mathrm{~mW} / \mathrm{mK}^{2}$ at the same temperature shown in figure 56. 


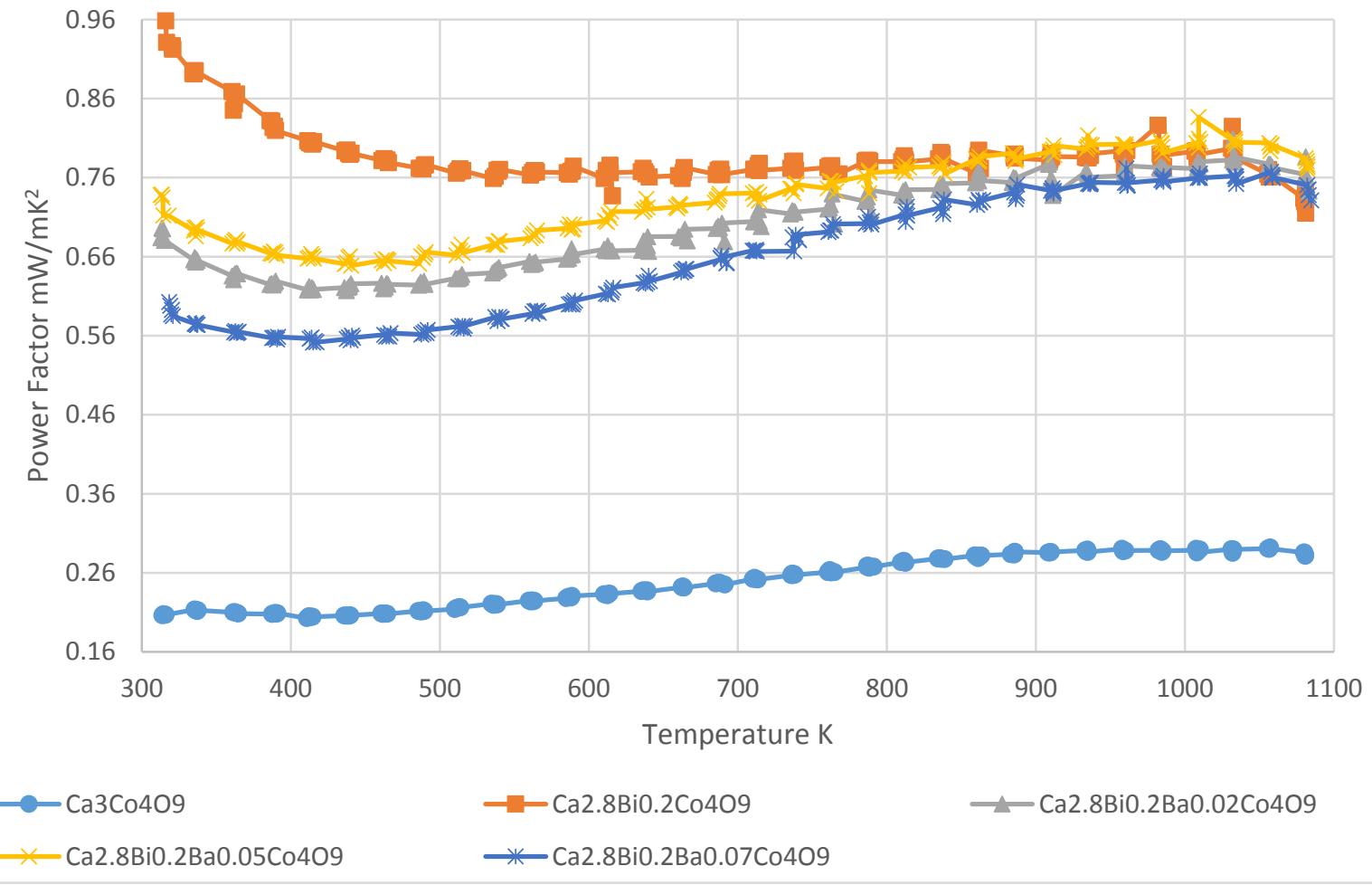

Figure 56 Temperature dependence of power factor for $\mathrm{Ca}_{2.8} \mathrm{Bi}_{0.2} \mathrm{Ba}_{4} \mathrm{Co}_{4} \mathrm{O}_{9}$ bismuth cation substitution \& barium non-stoichiometric addition where $u=0.0,0.02,0.05$, and 0.07 .

\subsection{Summary of results for cation substitution \& non-stoichiometric addition dual doping of $\mathrm{Ca}_{3} \mathrm{Co}_{4} \mathrm{O}_{9}$}

The dual doped samples achieved a low electrical resistivity of $\sim 40 \mu \Omega \mathrm{m}$ in all temperature ranges. This low electrical resistivity coupled with an enhanced Absolute Seebeck coefficient allowed for a high power factor of $0.84 \mathrm{~mW} / \mathrm{mK}^{2}$ at $1010 \mathrm{~K}$ with the optimal sample $\mathrm{Ca}_{2.8} \mathrm{Bi}_{0.2} \mathrm{Ba}_{0.05} \mathrm{C}_{4} \mathrm{O}_{9}$. This overlaps the bismuth cation substitution sample and is about three times greater than the $0.29 \mathrm{~mW} / \mathrm{mK}^{2}$ power factor of the pure $\mathrm{Ca}_{3} \mathrm{Co}_{4} \mathrm{O}_{9}$ at the same temperature. This was mainly due to the consistent low electrical 
resistivity across the whole temperature range, caused by the semi-conductive abilities of barium, along with the increase in room temperature Absolute Seebeck coefficient, caused by the grain boundary segregation of both dopants. Only the material's electrical properties were studied for this set since the power factor that was achieved was not greater than that of the bismuth cation substitution alone. 


\subsection{References}

1 Marat Gunes, Macit Ozenbas. Effect of grain size and porosity on phonon scattering enhancement of $\mathrm{Ca}_{3} \mathrm{C}_{4} \mathrm{O}_{9}$. Journal of Alloys and Compounds Vol.626, pp.360-367, 2015.

2 Boyle, Cullen; Calvillo, Paulo; Chen, Yun; Barbero, Ever J.; Mclntyre, Dustin. Grain boundary segregation and thermoelectric performance enhancement of bismuth doped calcium cobaltite. Journal of the European Ceramic Society 36(3):601-607 Elsevier 2016 0955-2219

3 Masashi Mikami, Kanju Chong, Yuzuru Miyazaki, Tsuyoshi Kajitani, Takahiro Inoue, Satoshi Sodeoka, and Ryoji Funahashi. Bi-Substitution effects on crystal structure and thermoelectric properties of $\mathrm{Ca}_{3} \mathrm{C}_{4} \mathrm{O}_{9}$ single crystals. Japanese Journal of Applied Physics. Vol.45, No.5A, pp.4131-4136, 2006.

4 Live Science Staff. Facts about Bismuth. September 5, 2013.

5 Tao Wu, Trevor A. Tyson, Haiyan Chen, Jianming Bai, Hsin Wang and Cherno Jaye. A structural phase transition in $\mathrm{Ca}_{3} \mathrm{Co}_{4} \mathrm{O}_{9}$ Associated with enhanced high temperature thermoelectric properties. Cornell University Library. April 15, 2012.

6 T. Takeuchi, T. Kondo, K. Soda, U. Mizutani, R. Funahashi, M. Shikano, S. Tsuda, T. Yokoya, S. Shin, T. Muro. Electronic structure and large thermoelectric power in $\mathrm{Ca}_{3} \mathrm{C}_{4} \mathrm{O}_{9}$. Journal of Electron Spectroscopy and Related Phenomena. Vol.137-140, pp.595-599, 2004.

7 Guangkun Ren, Jinle Lan, Chengcheng Zeng, Yaochun Liu, Bin Zhan, Sajid Butt, YuanHua Lin, and Ce-Wen Nan. High performance oxides-based thermoelectric materials. Journal of Minerals, Metals \& Materials Society. Vol.67, No.1, pp.211-221, 2015.

8 Calvillo, Paulo. Chen, Yun. Boyle, Cullen. Barnes, Paul. N. Song, Xueyan. Thermoelectric performance enhancement of calcium cobaltite through barium grain boundary segregation. Inorganic Chemistry. American Chemical Society Publications. September 11, 2015.

9 T. Sun, H. H. Hng, Q. Y. Yan, and J. Ma. Enhanced high temperature thermoelectric properties of $\mathrm{Bi}$-doped $\mathrm{c}$-axis oriented $\mathrm{Ca}_{3} \mathrm{C}_{4} \mathrm{O}_{9}$ thin films by pulsed laser deposition. Journal of Applied Physics. Vol.108, 2010.

10 Sajid Butt, Yaoyu Ren, Muhammad Umer Farooq, Bin Zhan, Rizwan ur Rahman Sagar, Yuanhua Lin, Ce-Web Nan. Enhanced thermoelectric performance of heavy-metals (M: $\mathrm{Ba}, \mathrm{Pb})$ doped misfit-layered ceramics: $\left(\mathrm{Ca}_{2}-\mathrm{M}_{\mathrm{x}} \mathrm{CoO}_{3}\right)_{0.62}\left(\mathrm{CoO}_{2}\right)$. Energy Conversion and Management. Vol.83, pp.35-41, 2014.

11 Ruoming Tian, Tianshu Zhang, Dewei Chu, Richard Donelson, Li Tao, Sean Li. Enhancement of high temperature thermoelectric performance in $\mathrm{Bi}, \mathrm{Fe}$ co-doped layered oxide-based material $\mathrm{Ca}_{3} \mathrm{C}_{0} \mathrm{O}_{9+\delta}$. Journal of Alloys and Compounds. Vol.615, pp.311-315, 2014. 
12 Q. M. Lu, J. X. Zhang, Q. Y. Zhang, Y. Q. Liu, and D. M. Liu. Improved thermoelectric properties of $\mathrm{Ca}_{3-\mathrm{x}} \mathrm{Ba}_{x} \mathrm{CO}_{4} \mathrm{O}_{9}(\mathrm{x}=0 \sim 0.4)$ bulks by sol-gel and SPS method. $15^{\text {th }}$ International Conference of Thermoelectrics. 2006.

${ }^{13}$ Ankam Bhaskar, Z. -R. Lin, Chia-Jyi Liu. Thermoelectric properties of Ca2.95Bio.05Co4${ }_{x} \mathrm{Fe}_{x} \mathrm{O}_{9+\delta}(0<\mathrm{x}<0.15)$. Energy Conversion and Management. Vol.76, pp.63-67, 2013.

14 Haoshan Hao, Qinglin He, ChangQing Chen, Hongwei Sun, and Xing Hu. Textured structure and anisotropic thermoelectric properties of $\mathrm{Ca}_{2.7} \mathrm{Bi}_{0.3} \mathrm{C}_{0} \mathrm{O}_{9}$ oxide prepared by conventional solid-state reaction. International Journal of Modern Physics B. Vol.23, No.1, pp.87-95, 2009.

15 Siwen Li, Ryoji Funahashi, Ichiro Matsubara, Kazuo Ueno, Satoshi Sodeoka, and Hiroyuki Yamada. Synthesis and thermoelectric properties of the new oxide materials

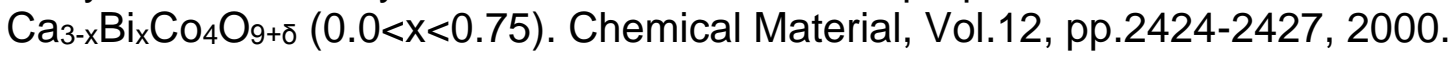

${ }^{16}$ Zhang Feipeng, Lu Qingmei, Li Tingxian, Zhang Xin, Zhang Jiuxing, Song Xiaoyan. Preparation and thermoelectric transport properties of $\mathrm{Ba}-$, La-, and Ag- doped $\mathrm{Ca}_{3} \mathrm{Co}_{4} \mathrm{O}_{9}$ oxide materials. Journal of Rare Earths. Vol.31, No.8, pp.778-783, 2013.

${ }^{17}$ Haoshan Hao, Limin Zhao, and Xing Hu. Microstructure and thermoelectric properties of $\mathrm{Bi}$-and $\mathrm{Cu}$-substituted $\mathrm{Ca}_{3} \mathrm{CO}_{4} \mathrm{O}_{9}$ oxides. Journal of Materials Science technology, Vol.25, pp.105-108, 2009.

18 G. Constantinescu, Sh. Rasekh, M. A. Torres, M. A. Madre, A. Sotelo, J. C. Diez. Improvement of thermoelectric properties in $\mathrm{Ca}_{3} \mathrm{C}_{4} \mathrm{O}_{9}$ ceramics by $\mathrm{Ba}$ doping. Journal of Materials Science., Vol.26, pp.3466-3473, 2015.

19 C. S. Huang, F. P. Zhang, X. Zhang, Q.M. Lu, J. X. Zhang, Z. Y. Liu. Enhanced thermoelectric figure of merit through electrical and thermal transport modulation by dual-doping and texture modulating for $\mathrm{Ca}_{3} \mathrm{Co}_{4} \mathrm{O}_{9+\delta}$ oxide materials. Journal of Alloys and Compounds. Vol.687, pp.87-94, 2016. 


\section{Chapter 9:}

\section{Optimization of Dual Doping $\mathrm{Ca}_{3} \mathrm{Co}_{4} \mathrm{O}_{9}$ by Controlling Grain Growth \& Texture while Enhancing Absolute Seebeck Coefficient}

\subsection{Motivation}

In the last two chapters the dual doping method has been investigated with two different approaches. The first was through non-stoichiometric addition of both dopants and the second was through cationic substitution of one dopant and non-stoichiometric addition of the second. The second approach seems to over dope less easily and holds more promise. This is the method concentrated on and analyzed in this chapter. Chapter 8 showed that using $\mathrm{Ca}_{2.8} \mathrm{Bi}_{0.2} \mathrm{Ba}_{4} \mathrm{C}_{4} \mathrm{O}_{9}$ concentration of bismuth caused mild over doping. This lead to the motivation of this chapter where a lower concentration of bismuth will be used. Therefore, the baseline was doped with a set concentration of bismuth by using the cation substitution method as before, where the bismuth atoms will replace calcium atoms. The concentration used was bismuth $\mathrm{x}=0.1$ to avoid over doping. Barium used the method of non-stoichiometric addition allowing it to deposit itself freely. Since barium's optimization was reached at barium 0.05 addition, the doping levels used for barium were $0.02,0.05,0.07$, and 0.1 , in order to find a trend and see if the Absolute Seebeck Coefficient can be further enhanced and grain texture improved thus enhancing the power factor and overall efficiency. 


\subsection{Experimental Procedure}

$\mathrm{Ca}_{3-\mathrm{x}} \mathrm{Bi}_{\times} \mathrm{Ba}_{4} \mathrm{Co}_{4} \mathrm{O}_{9}$ powders, where $\mathrm{x}=0.0 \& 0.1$ and $\mathrm{u}=0.0,0.02,0.05,0.07$ were obtained through a sol-gel route in which stoichiometric ratios of $\mathrm{Ca}\left(\mathrm{NO}_{3}\right)_{2} \bullet 4 \mathrm{H}_{2} \mathrm{O}$, $\mathrm{Co}\left(\mathrm{NO}_{3}\right)_{2} \cdot 6 \mathrm{H}_{2} \mathrm{O}$ and $\mathrm{Bi}\left(\mathrm{NO}_{3}\right)_{2} \cdot 5 \mathrm{H}_{2} \mathrm{O}$ were mixed with non-stoichiometric ratios of $\mathrm{Ba}\left(\mathrm{NO}_{3}\right)_{2}$ in deionized water. Ethylene glycol and polyethylene glycol were used to aid polymerization of the solution and to aid in particle size. ${ }^{[1]}$ Lastly, nitric acid was added to induce nitrate salts decomposition and facilitate new compound formation. ${ }^{[2]}$

The liquid solution was then submerged in a silicone oil bath on a hot plate and mechanically stirred at $353 \mathrm{~K}$ for 3 hours to achieve the sol-gel state. The sol-gel was then distributed to ceramic crucibles loosely covered with aluminum foil and put into a box furnace with a ramp rate of $10 \mathrm{~K}$ per minute and held at $773 \mathrm{~K}$ for 2 hours then cooled at a rate of $10 \mathrm{~K}$ per minute. Holding the temperature here, at $773 \mathrm{~K}$, for the allotted time will ensure that any inorganic material is removed from the material. The ashes were then suspended in ethanol and ball milled for 20 minutes alternating between 1 minute of milling and 1 minute of resting in order to aid in the dissipation of heat. The powders were deposited in a tubular furnace where they were calcined in an oxygen rich induced environment with a ramp rate of $10 \mathrm{~K}$ per minute and held at $973 \mathrm{~K}$ for 4 hours, to achieve the $\mathrm{Ca}_{3} \mathrm{Co}_{4} \mathrm{O}_{9}$ crystal lattice. The powders were then cooled down to room temperature at a rate of $10 \mathrm{~K}$ per minute.

Two pellets were pressed, one to test its electrical properties and the other for its thermal properties. The calcined powders were pressed uniaxially with $1 \mathrm{GPa}$ of force 
and held for 10 minutes at $298 \mathrm{~K}$. The pellets' densities were obtained before they were put in to a tubular furnace to be sintered. The sintering process removes any extra moisture the pellet may contain and assures proper grain alignment. Pellets were sintered in an oxygen rich induced environment with a ramp rate of $10 \mathrm{~K}$ per minute and held at $1233 \mathrm{~K}$ for 9 hours. It was then cooled back to room temperature at $4 \mathrm{~K}$ per minute and once again the densities were taken. Pellets were cut into a $2 \mathrm{~mm} \times 3.5 \mathrm{~mm} \times 9 \mathrm{~mm}$ rectangular shapes and inserted into a Linseis LSR 3-Seebeck machine, subjected to a low pressure helium, He, environment where the pellet's electrical resistivity and Absolute Seebeck Coefficient were simultaneously determined by means of a dc four-probe method. Since helium is an inert gas it will not react or interfere with the measurement. A second pellet was cut along a different axis and inserted into the Linseis LFA 1000 machine to test its thermal conductivity. The pellet was cut to be $2 \mathrm{~mm}$ thick and polished to a diameter of $11-13 \mathrm{~mm}$. Any less than $11 \mathrm{~mm}$ and there would be room for heat to escape the holder, any more than $13 \mathrm{~mm}$ and the pellet would be too large for the holder. The thermal conductivity is calculated by the equation

$$
\mathrm{K}=\lambda \mathrm{C}_{\mathrm{p} \rho \mathrm{m}}
$$

Where $\lambda$ is the thermal diffusity in meters squared per second $\left(\mathrm{m}^{2} / \mathrm{s}\right), \mathrm{C}_{\mathrm{p}}$ is the specific heat capacity in joules per kilogram kelvin $(\mathrm{J} / \mathrm{kg} \cdot \mathrm{K})$, and $\rho_{\mathrm{m}}$ is the mass density in grams per centimeter cubed $\left(\mathrm{g} / \mathrm{cm}^{3}\right)$. As stated in the first section, these are the three values needed in order to obtain the materials' ZT value. Bismuth cation substitution has been reported on and a concentration of 0.1 was used and held constant throughout the varied 
barium dopings. A non-stoichiometric addition method was used for varying the doping concentrations of barium. Their chemistries are as follows: $\mathrm{Ca}_{3-\mathrm{x}} \mathrm{Bi}_{\times} \mathrm{Ba}_{4} \mathrm{Co}_{4} \mathrm{O}_{9}$ where $\mathrm{x}=$ $0.0,0.1$ and $u=0.0,0.02,0.05,0.07$, and 0.1 .

\subsection{Decreased electrical resistivity and enhanced thermoelectric properties of $\mathrm{Ca}_{3} \mathrm{Co}_{4} \mathrm{O}_{9}$ induced by dual doping}

9.3.1 Low electrical resistivity achieved through dual doping

It is clearly evident in figure 57 that the $\mathrm{Ca}_{2.9} \mathrm{Bi}_{0.1} \mathrm{Ba}_{0.07} \mathrm{Co}_{4} \mathrm{O}_{9}$ doped sample has a significantly lower room temperature electrical resistivity than that of the $\mathrm{Ca}_{2.9} \mathrm{Bi}_{0.1} \mathrm{Co}_{4} \mathrm{O}_{9}$. At $315 \mathrm{~K}$ the bismuth barium dual doped sample has an electrical resistivity of $\sim 28 \mu \Omega \mathrm{m}$ whereas the $\mathrm{Ca}_{2.9} \mathrm{Bi}_{0.1} \mathrm{Co}_{4} \mathrm{O}_{9}$ is $\sim 48 \mu \Omega \mathrm{m}$ at the same temperature. As the bismuth cation substitution sample increases in temperature its electrical resistivity experiences a large increase as well, from $\sim 48 \mu \Omega m$ up to $\sim 74 \mu \Omega m$ at around $1080 \mathrm{~K}$, yet the $\mathrm{Ca}_{2.9} \mathrm{Bi}_{0.1} \mathrm{Ba}_{0.07} \mathrm{C}_{4} \mathrm{O}_{9}$ sample only increases slightly from $\sim 28 \mu \Omega \mathrm{m}$ to $\sim 38 \mu \Omega \mathrm{m}$. ${ }^{[3,4]}$ This low electrical resistivity can be attributed to the exceptionally low electrical resistivities that bismuth and barium can achieve independently. This sample has a slightly higher electrical resistivity than the bismuth $\mathrm{x}=0.2$ in chapters $4 \& 8$ but the bismuth $\mathrm{x}=0.2$ rises from $22 \mu \Omega m$ to about $49 \mu \Omega m$ which is larger than the optimal dual doped sample with the lower bismuth concentration.

All samples dual doped with bismuth and barium had a comparable or lower electrical resistivity than the sample doped only through bismuth cation substitution, with the exception of the over doped sample of $\mathrm{Ca}_{2.9} \mathrm{Bi}_{0.1} \mathrm{Ba}_{0.1} \mathrm{Co}_{4} \mathrm{O}_{9}$. Each dual doped sample 
maintained this low electrical resistivity into the high temperatures as the sample doped only with bismuth cation substitution continued to rise as the temperature increased. The optimal concentration of $\mathrm{Ca}_{2.9} \mathrm{Bi}_{0.1} \mathrm{Ba}_{0.07} \mathrm{C}_{04} \mathrm{O}_{9}$ shown in figure 57 achieved a low electrical resistivity of $28 \mu \Omega m$ at $315 \mathrm{~K}$ and $\sim 37 \mu \Omega \mathrm{m}$ at $982 \mathrm{~K}$ which is $35 \%$ of that of the pure undoped $\mathrm{Ca}_{3} \mathrm{Co}_{4} \mathrm{O} 9$.

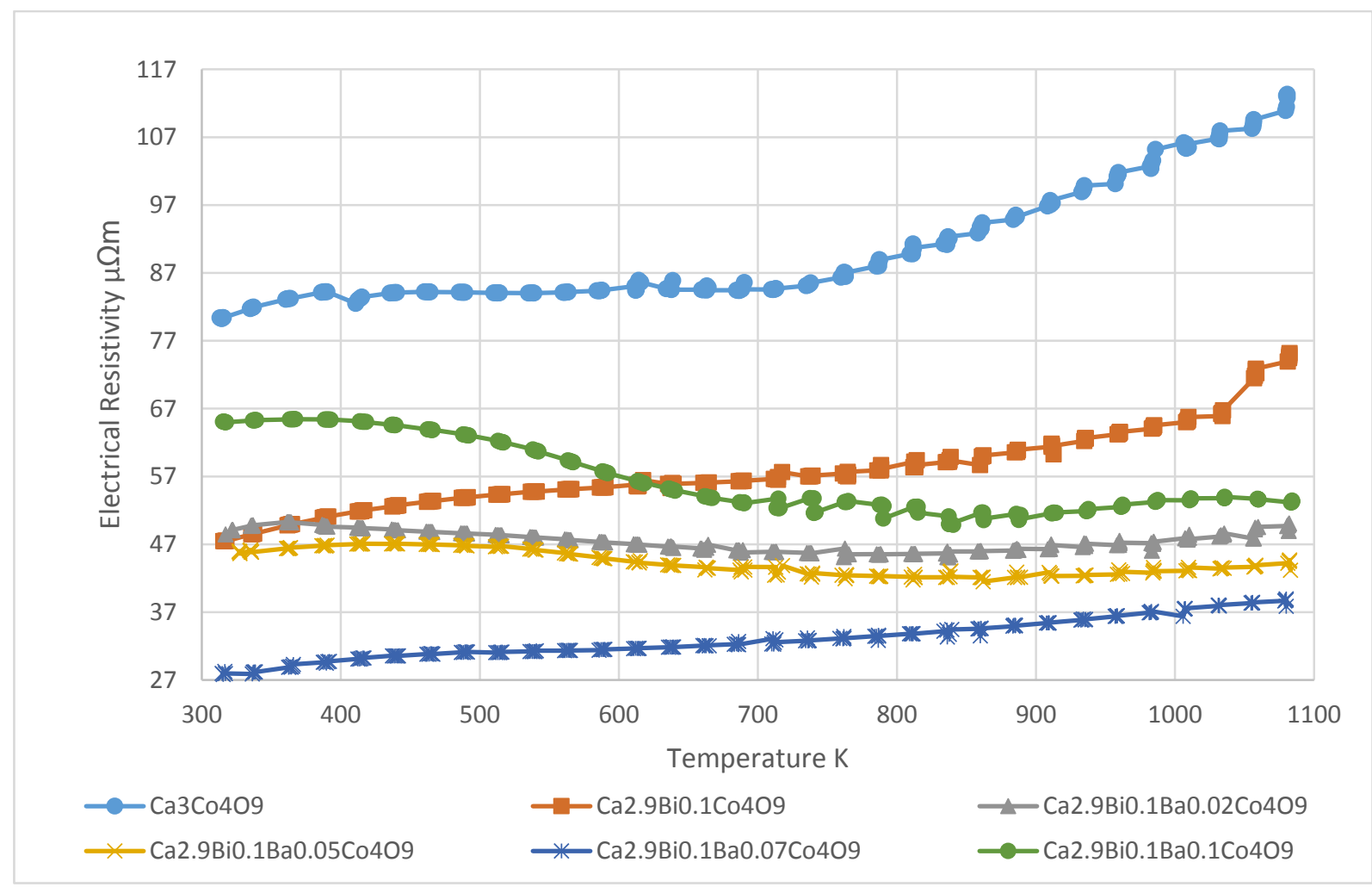

Figure 57 Temperature dependence of electrical resistivity for $\mathrm{Ca}_{2.9} \mathrm{Bi}_{0.1} \mathrm{Ba}_{4} \mathrm{Co}_{4} \mathrm{O}_{9}$ bismuth cation substitution \& barium non-stoichiometric addition where $u=0.0,0.02$, $0.05,0.07$, and 0.1 .

9.3.2 Fine tuning electrical resistivity and Seebeck coefficient through dual dopants.

Both the bismuth and the barium were able to act in concert to vastly improve the Absolute Seebeck Coefficient of this material. When only bismuth is introduced the 
Absolute Seebeck coefficient increases to around $140 \mu \mathrm{V} / \mathrm{k}$ at $315 \mathrm{~K}$. However, once the barium is introduced the material experiences another, even greater, enhancement of its Absolute Seebeck Coefficient above $160 \mu \mathrm{V} / \mathrm{k}$ at $315 \mathrm{~K}$, due to its segregation at the grain boundary. ${ }^{[5,6]}$ The Absolute Seebeck coefficient in the high temperature range is also comparable to that of the sample solely doped with bismuth cation substitution, demonstrated in figure 58, whereas the dual doped samples, in previous chapters, had a lower value than the bismuth alone. After increasing the barium's levels to 0.1 it can be seen that the Absolute Seebeck coefficient flattens out at around $600 \mathrm{~K}$, which as mentioned previously is indicative of over doping. This over doping is also demonstrated by having a much larger resistivity trend than the rest of the samples. 


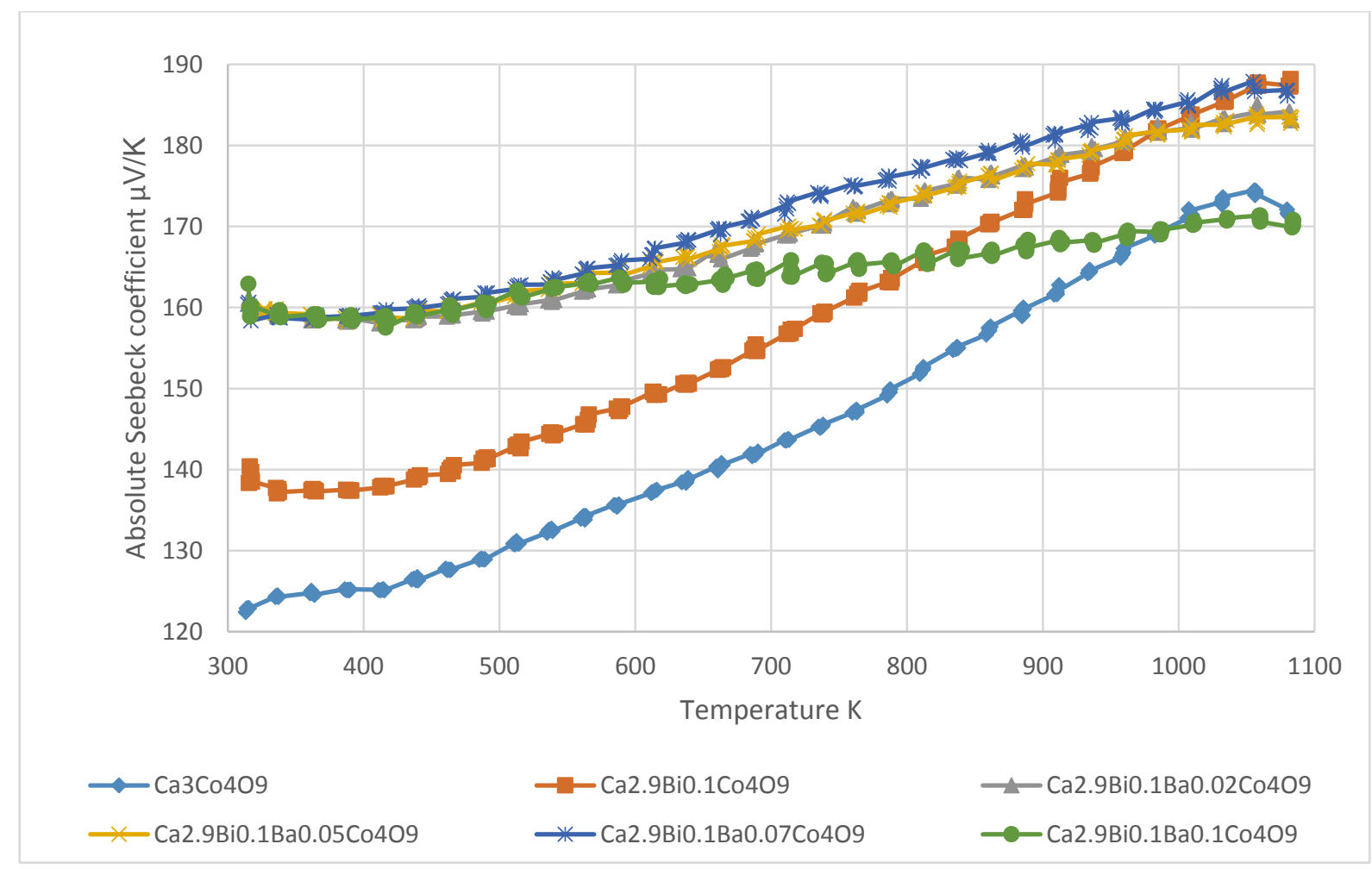

Figure 58 Temperature dependence of absolute seebeck coefficient for $\mathrm{Ca}_{2.9} \mathrm{Bi}_{0.1} \mathrm{Ba}_{4} \mathrm{C}_{4} \mathrm{O}_{9}$ bismuth cation substitution \& barium non-stoichiometric addition where $u=0.0,0.02,0.05,0.07$, and 0.1

This low electrical resistivity and large Absolute Seebeck Coefficient achieved by the optimal $\mathrm{Ca}_{2.9} \mathrm{Bi}_{0.1} \mathrm{Ba}_{0.07} \mathrm{Co}_{4} \mathrm{O}_{9}$ chemistry across all temperature ranges leads to an enhanced power factor in all temperature ranges as well. The $\mathrm{Ca}_{2.9} \mathrm{Bi}_{0.1} \mathrm{Ba}_{0.07} \mathrm{C}_{4} \mathrm{O}_{9}$ sample yields a high power factor of $0.93 \mathrm{~mW} / \mathrm{mK}^{2}$ at $315 \mathrm{~K}$ and $0.94 \mathrm{~mW} / \mathrm{mK}^{2}$ at $1005 \mathrm{~K}$ both of which are nearly 5 times and over 3 times the pure undoped $\mathrm{Ca}_{3} \mathrm{Co}_{4} \mathrm{O}_{9}$ sample, respectively. This is shown in figure 59 and is one of the highest power factors reported on for this material. 


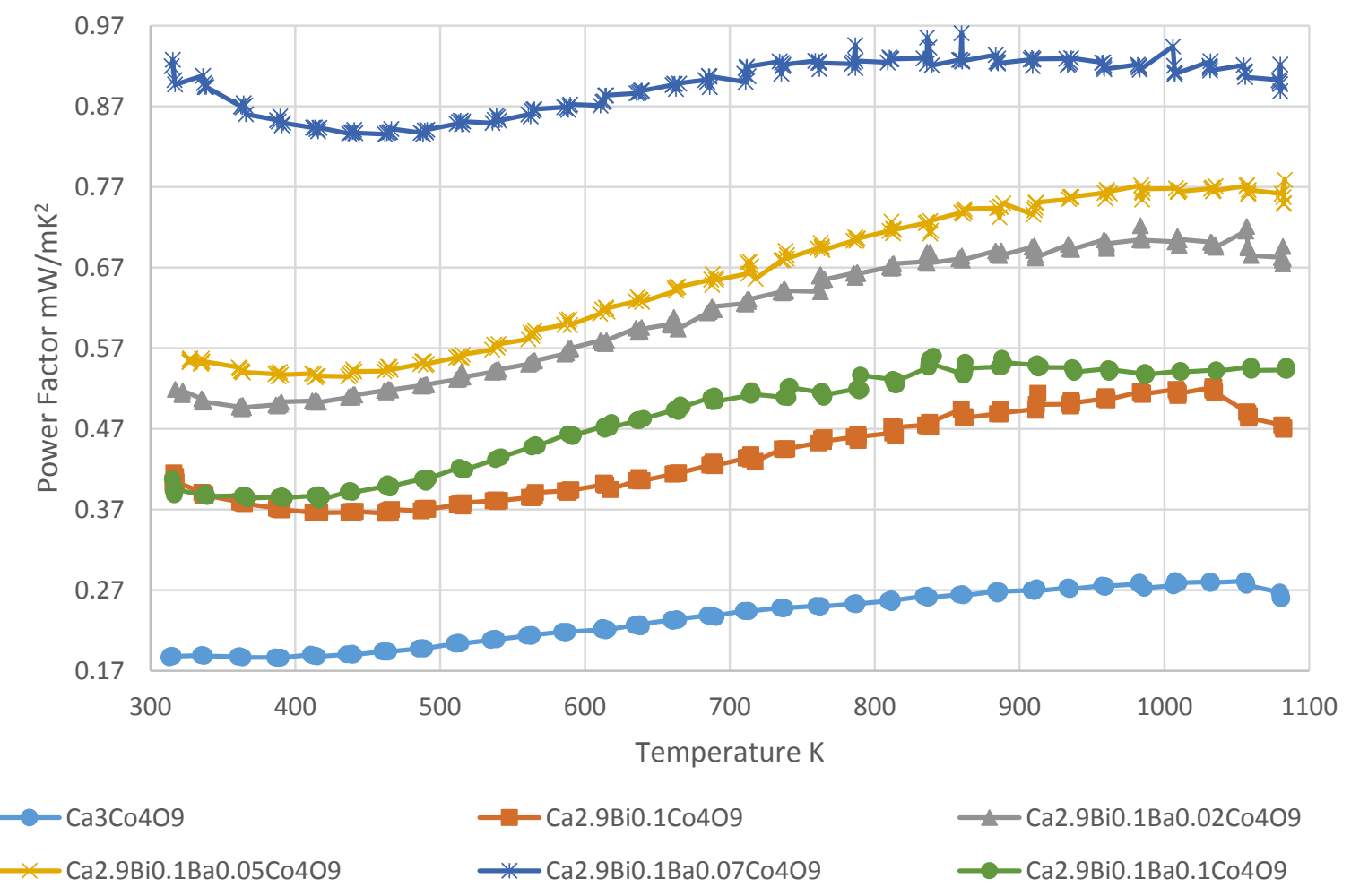

Figure 59 Temperature dependence of power factor for $\mathrm{Ca}_{2.9} \mathrm{Bi}_{0.1} \mathrm{Ba}_{4} \mathrm{Co}_{4} \mathrm{O}_{9}$ bismuth cation substitution \& barium non-stoichiometric addition where $u=0.0,0.02,0.05,0.07$, and 0.1 .

\subsection{Microstructure of dual doped Bismuth \& Barium}

\subsubsection{Structure and lattice parameters}

Figure 60 shows the X-Ray diffraction patterns obtained from ground powders under ambient conditions. Table 3 shows the lattice parameters of these samples for their different chemistries. The samples containing bismuth had shifted peaks to lower angles indicating an increase in lattice parameters thus reassuring the entry of bismuth into the lattice. Barium however, has little effect on the parameters as its concentration increases, 
since it does not deposit into the grain interior, leaving the parameters to only deviate based on the small variations of bismuth from sample to sample. These peaks in figure 60 can be indexed as the $\mathrm{Ca}_{3} \mathrm{Co}_{4} \mathrm{O}_{9}$ monoclinic structure with the $\mathrm{x}=0.0, \mathrm{u}=0.0$ sample showing little to no trace of $\mathrm{Co}_{3} \mathrm{O}_{4}$ which can be seen from the $\mathrm{Co}_{3} \mathrm{O}_{4}(311)$ peak indexed.

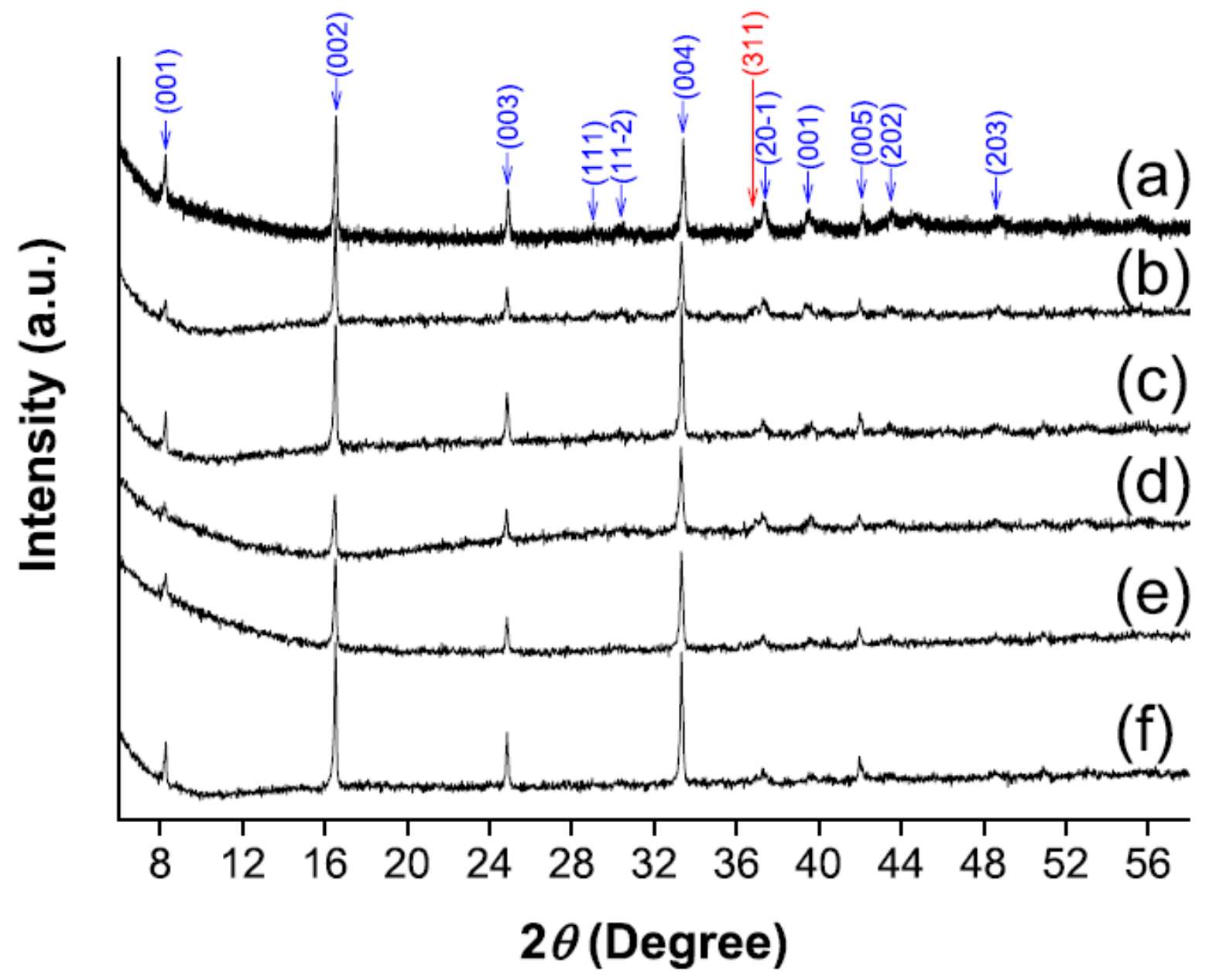

Figure $60 X R D$ powder diffraction patterns for the $\mathrm{Ca}_{3-x} \mathrm{Bi}_{x} \mathrm{Ba}_{4} \mathrm{Co}_{4} \mathrm{O}_{9}$ samples. [6] 
Table 3 Lattice parameters for samples with different chemistries ${ }^{[6]}$

\begin{tabular}{|c|c|c|c|c|c|c|c|c|}
\hline $\mathbf{B i}$ & $\mathrm{Ba}$ & $\mathbf{a}(\AA)$ & $\mathbf{b}_{1}(\mathbf{A})$ & $c(\AA)$ & $\alpha^{\circ}$ & $\beta^{\circ}$ & $\mathrm{Y}^{\circ}$ & $\mathbf{b}_{2}(\AA)$ \\
\hline$x=0$ & $y=0$ & 4.8637 & 4.5540 & 10.8300 & 90 & 98.45 & 90 & 2.7864 \\
\hline$x=0.1$ & $y=0$ & 4.8747 & 4.5494 & 10.8607 & 90 & 98.77 & 90 & 2.7903 \\
\hline$x=0.1$ & $y=0.02$ & 4.8810 & 4.5456 & 10.8646 & 90 & 98.91 & 90 & 2.7900 \\
\hline$x=0.1$ & $y=0.05$ & 4.8921 & 4.5512 & 10.8765 & 90 & 98.93 & 90 & 2.7878 \\
\hline$x=0.1$ & $y=0.07$ & 4.9290 & 4.5625 & 10.8791 & 90 & 99.38 & 90 & 2.7767 \\
\hline$x=0.1$ & $y=0.1$ & 4.8527 & 4.5606 & 10.8415 & 90 & 98.09 & 90 & 2.7929 \\
\hline
\end{tabular}

\subsubsection{Texture and Grain Alignment}

Fractured samples were analyzed using a Scanning Electron Microscope (SEM) which show the grain growth and crystal texture development in figure 61 . The presence of bismuth triggered grain growth, improved texture, and grain alignment. Grain texture and alignment continue to improve in the presence of barium. The optimal chemistry $\mathrm{Ca}_{2.9} \mathrm{Bi}_{0.1} \mathrm{Ba}_{0.07} \mathrm{CO}_{4} \mathrm{O}_{9}$ is shown in image (c) and it is evident that the grain alignment not only has improved from the sample with bismuth alone but vastly improved from the undoped $\mathrm{Ca}_{3} \mathrm{Co}_{4} \mathrm{O}_{9}$ sample. Image (d) shows the over doped sample as its alignment begins to decrease. 

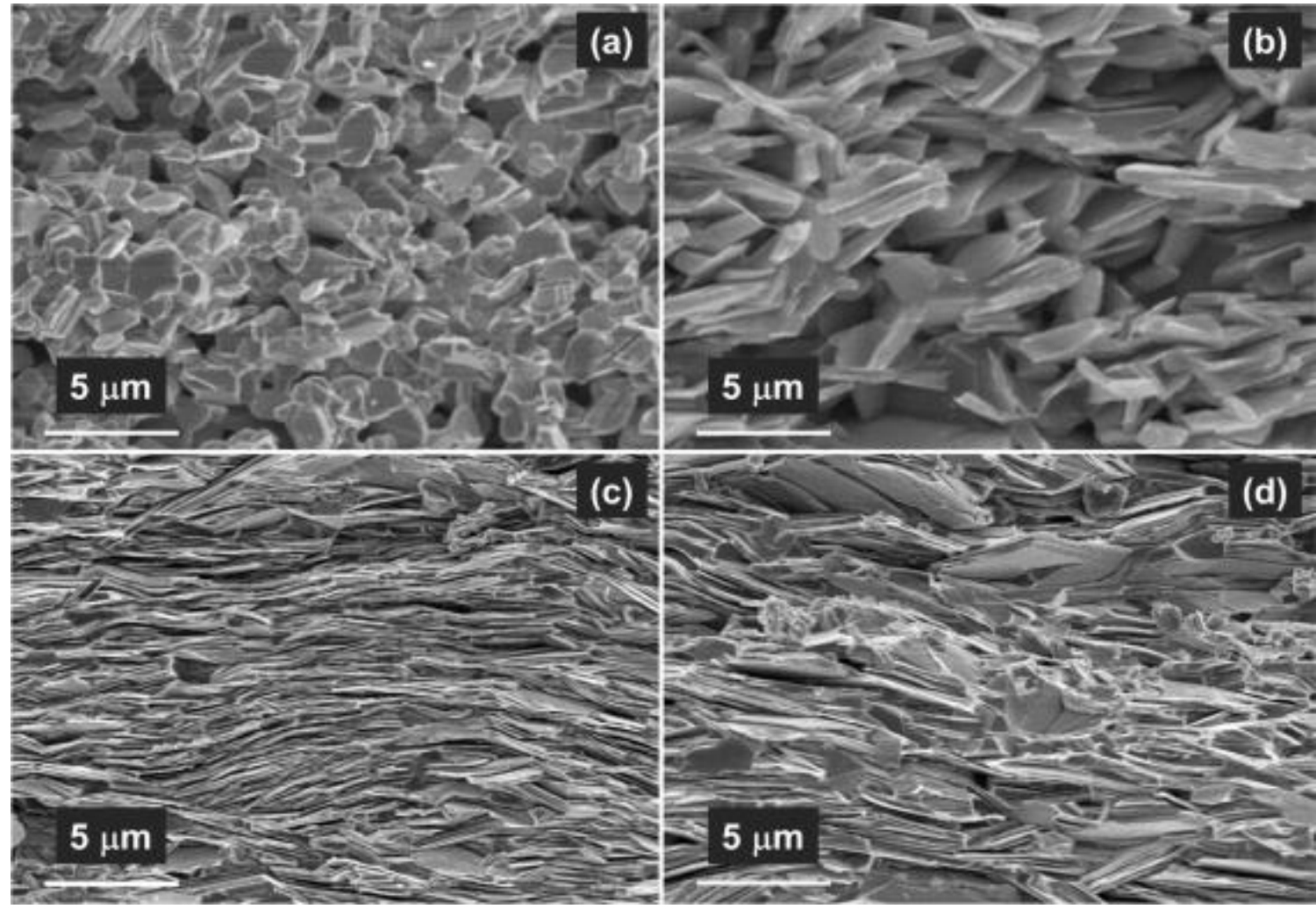

Figure 61 SEM images showing crystal texture development of the $\mathrm{Ca}_{3} \mathrm{Co}_{4} \mathrm{O}_{9}$ samples with dual element doping of bismuth and barium simultaneously. [6]

To further elucidate this grain alignment concept, figure 62 shows the microstructure progression from the undoped sample to that of the $\mathrm{Ca}_{2.9} \mathrm{Bi}_{0.1} \mathrm{Ba}_{0.07} \mathrm{Co}_{4} \mathrm{O}_{9}$. It also demonstrates how the barium deposits at the grain boundary while bismuth improved the crystal texture by entering the grains. 


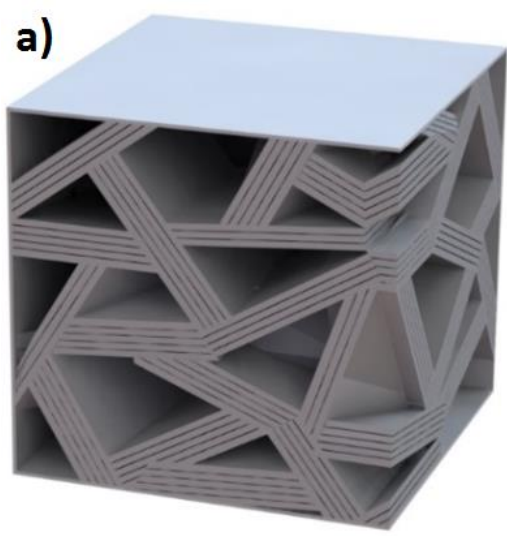

$\mathrm{Ca}_{3} \mathrm{Co}_{4} \mathrm{O}_{9}$ b)

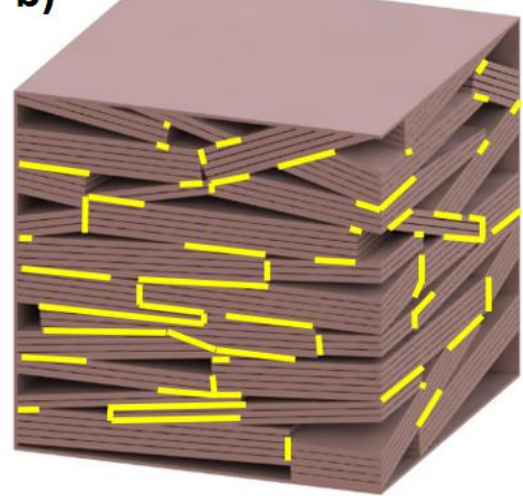

$\mathrm{Ca}_{2.9} \mathrm{Bi}_{0.1} \mathrm{Ba}_{0.07} \mathrm{Co}_{4} \mathrm{O}_{9}$
Bismuth in grain interior

Barium at grain boundary

Figure 62 Microstructure drawing of undoped $\mathrm{Ca}_{3} \mathrm{Co}_{4} \mathrm{O}_{9}$ and $\mathrm{Ca}_{2.9} \mathrm{Bi}_{0.1} \mathrm{Ba}_{0.07} \mathrm{Co}_{4} \mathrm{O}_{9}$.

\subsection{Nanostructure analysis of dual doped Bismuth \& Barium through use of TEM}

Transmission Electron Microscope (TEM) was used to explore the diffraction contrast and gain knowledge of the chemical composition of the $\mathrm{Ca}_{2.9} \mathrm{Bi}_{0.1} \mathrm{Co}_{4} \mathrm{O}_{9}$ sample detailed in figure 63. Image (a) shows the diffraction contrast of the $\mathrm{Ca}_{2.9} \mathrm{Bi}_{0.1} \mathrm{Co}_{4} \mathrm{O}_{9}$ cation substitution sample. EDS values, shown in table 4, were taken from the points shown in figure 63 image (a), once again demonstrating bismuth's ability to deposit into the lattice and at the grain boundary. Image (b) shows no evidence of a secondary phase and the red arrow indicates the bismuth enriched grain boundary. 

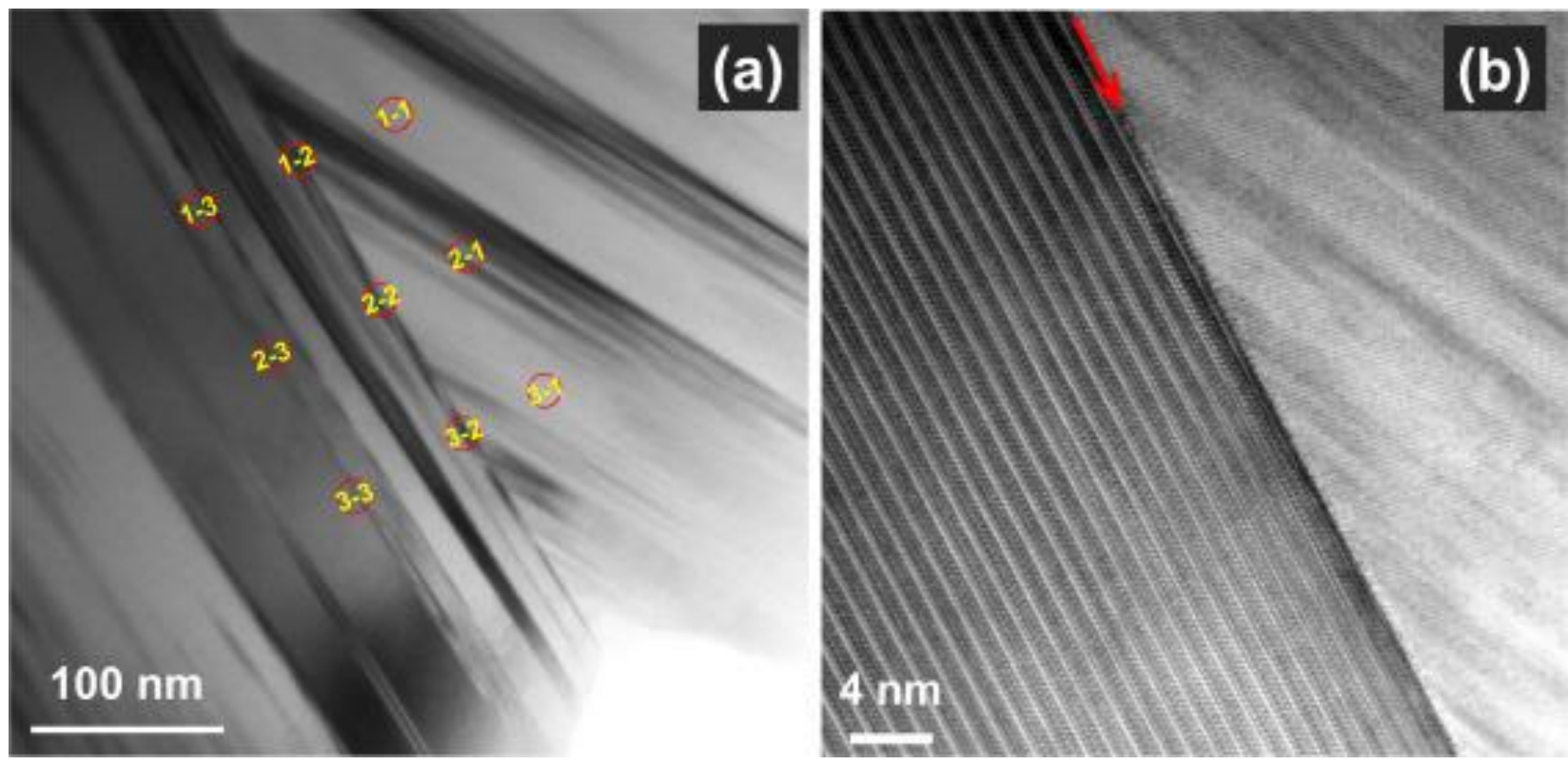

Figure 63 (a) Diffraction contrast image and (b) high resolution TEM from the $\mathrm{Ca}_{2.9} \mathrm{Bi}_{0.1} \mathrm{CO}_{4} \mathrm{O}_{9}$ sample. ${ }^{[6]}$

Table 4 Chemical compositions of two neighboring grains and grain boundary in between. [6]

\begin{tabular}{|l|lll|lll|lll|}
\hline Atomic \% & $\mathbf{1 - 1}$ & $\mathbf{1 - 2}$ & $\mathbf{1 - 3}$ & $\mathbf{2 - 1}$ & $\mathbf{2 - 2}$ & $\mathbf{2 - 3}$ & $\mathbf{3 - 1}$ & $\mathbf{3 - 2}$ & $\mathbf{3 - 3}$ \\
\hline $\mathbf{O}$ & 61.32 & 59.09 & 59.62 & 66.58 & 61.03 & 56.11 & 64.82 & 63.07 & 62.16 \\
$\mathbf{C a}$ & 17.36 & 17.31 & 17.08 & 14.75 & 16.89 & 18.9 & 15.14 & 15.77 & 16.5 \\
Co & 20.8 & 22.94 & 22.66 & 18.15 & 21.36 & 24.41 & 19.61 & 20.58 & 20.85 \\
$\mathbf{B i}$ & 0.52 & 0.65 & 0.64 & 0.52 & 0.71 & 0.58 & 0.43 & 0.58 & 0.48 \\
\hline
\end{tabular}

Figure 64 (a) \& (b) show the nanostructure of the lamellar of the $\mathrm{Ca}_{2.9} \mathrm{Bi}_{0.1} \mathrm{Ba}_{0.07} \mathrm{Co}_{4} \mathrm{O}_{9}$ sample. EDS data, in table 5 , shows that there is a depletion of bismuth at the grain boundary. This is due to the presence of the barium. As the barium concentration increases the grain boundary becomes more and more enriched with 
barium and the bismuth depletes causing more to deposit into the lattice. Barium is also not present anywhere in the grain interior. Image (b) shows no secondary phase at the grain boundary plane. This is opposite to the intragrain lattice where the grain boundary region experiences some lattice distortions. ${ }^{[6]}$ This is most likely the direct result of the presence of barium at the boundary. Image (c) is a high resolution TEM image also showing no secondary phase at the grain boundary.
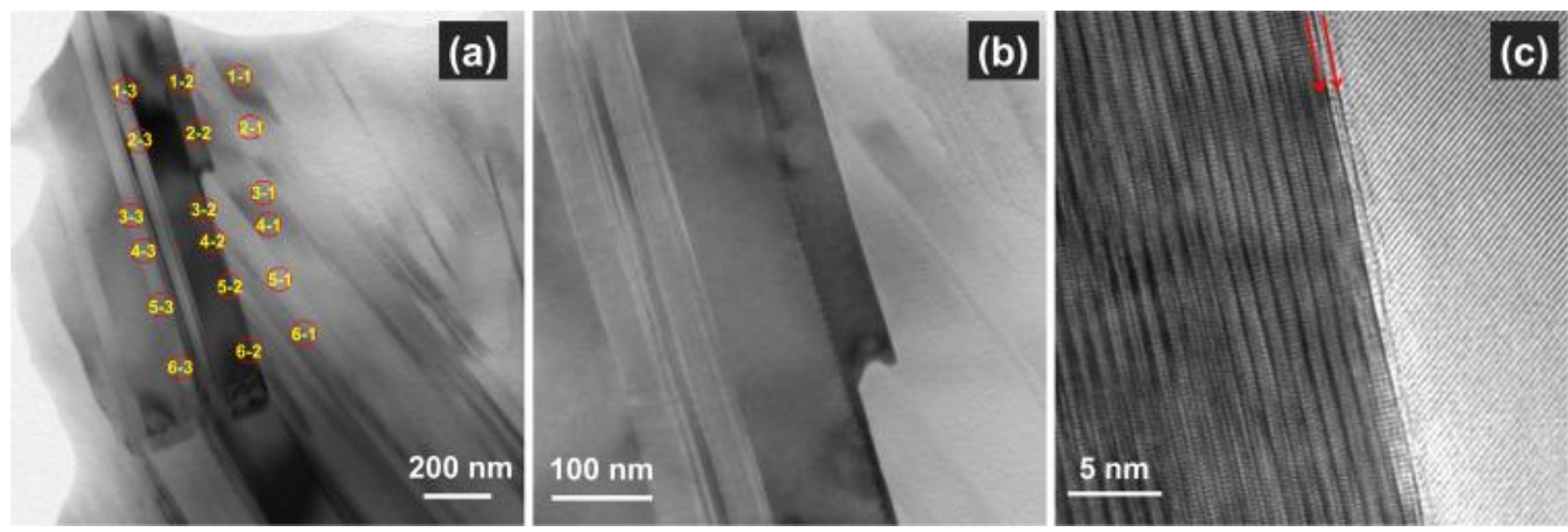

Figure 64 TEM images from the $\mathrm{Ca}_{2.9} \mathrm{Bi}_{0.1} \mathrm{Ba}_{0.07} \mathrm{Co}_{4} \mathrm{O}_{9}$ sample. ${ }^{[6]}$

Table 5 Chemical compositions of two neighboring grains and grain boundary in between for the $\mathrm{Ca}_{2.9} \mathrm{Bi}_{0.1} \mathrm{Ba}_{0.07} \mathrm{Co}_{4} \mathrm{O}_{9}$ sample. [6]

\begin{tabular}{|c|c|c|c|c|c|c|c|c|c|c|c|c|c|c|c|c|c|c|}
\hline $\mathbf{A t} \%$ & $\mathbf{1 - 1}$ & $\mathbf{1 - 2}$ & $\mathbf{1 - 3}$ & $\mathbf{2 - 1}$ & $\mathbf{2 - 2}$ & $\mathbf{2 - 3}$ & $\mathbf{3 - 1}$ & $\mathbf{3 - 2}$ & $\mathbf{3 - 3}$ & $\mathbf{4 - 1}$ & $\mathbf{4 - 2}$ & $\mathbf{4 - 3}$ & $\mathbf{5 - 1}$ & $\mathbf{5 - 2}$ & $\mathbf{5 - 3}$ & $\mathbf{6 - 1}$ & $\mathbf{6 - 2}$ & $\mathbf{6 - 3}$ \\
$\mathbf{0}$ & 67.9 & 62.7 & 58.0 & 68.2 & 59.7 & 62.5 & 66.5 & 67.0 & 62.1 & 68.8 & 65.3 & 60.0 & 65.8 & 64.2 & 59.5 & 59.6 & 63.0 & 60.9 \\
$\mathbf{C a}$ & 13.3 & 10.2 & 16.6 & 13.2 & 2.5 & 12.0 & 14.6 & 11.6 & 15.2 & 13.1 & 12.4 & 13.5 & 14.9 & 8.4 & 12.6 & 18.0 & 8.2 & 13.8 \\
$\mathbf{C o}$ & 18.5 & 24.4 & 24.8 & 18.1 & 30.9 & 25.2 & 18.4 & 19.6 & 22.1 & 17.7 & 20.5 & 26.2 & 18.8 & 24.5 & 27.5 & 21.9 & 25.4 & 24.8 \\
$\mathbf{B a}$ & 0.0 & 2.5 & 0.0 & 0.0 & 6.9 & 0.0 & 0.0 & 1.3 & 0.0 & 0.0 & 1.4 & 0.0 & 0.0 & 2.6 & 0.0 & 0.0 & 3.0 & 0.0 \\
$\mathbf{B i}$ & 0.4 & 0.2 & 0.6 & 0.5 & 0.1 & 0.3 & 0.6 & 0.4 & 0.6 & 0.5 & 0.4 & 0.4 & 0.5 & 0.3 & 0.4 & 0.6 & 0.4 & 0.4 \\
\hline
\end{tabular}




\subsection{Calculating the thermal conductivities and $\mathrm{ZT}$ of dual doped $\mathrm{Ca}_{3} \mathrm{Co}_{4} \mathrm{O}_{9}$}

Thermal conductivities of the two samples with the optimal power factor from figure 59 were tested and compared with those of the undoped $\mathrm{Ca}_{3} \mathrm{C}_{4} \mathrm{O}_{9}$ and the $\mathrm{Ca}_{2.9} \mathrm{Bi}_{0.1} \mathrm{Co}_{4} \mathrm{O}_{9}$ samples in figure 65 . Barium has the tendency to vastly increase the density of the bulk sample which in turn will decrease its thermal conductivity affecting its otherwise high power factor. This high thermal conductivity keeps this material from cresting the 0.5 figure of merit mark. The optimal sample of $\mathrm{Ca}_{2.9} \mathrm{Bi}_{0.1} \mathrm{Ba}_{0.07} \mathrm{Co}_{4} \mathrm{O}_{9}$ has a thermal conductivity of $2.8 \mathrm{~W} / \mathrm{mK}$ at $373 \mathrm{~K}$ and $2.2 \mathrm{~W} / \mathrm{mK}$ at $1073 \mathrm{~K}$ both values of which are higher than that of the pure undoped $\mathrm{Ca}_{3} \mathrm{C}_{4} \mathrm{O}_{9}$. A high power factor allows this material to yield a ZT of 0.41 and 0.45 at $973 \mathrm{~K}$ and $1073 \mathrm{~K}$ respectively shown in figure 66. This is a $60 \%$ increase from the $\mathrm{Ca}_{2.9} \mathrm{Bi}_{0.1} \mathrm{C}_{4} \mathrm{O}_{9}$ sample doped only with bismuth using the cation substitution method and 3 times higher than the undoped $\mathrm{Ca}_{3} \mathrm{C}_{4} \mathrm{O}_{9}$ sample at $1073 \mathrm{~K}$. 


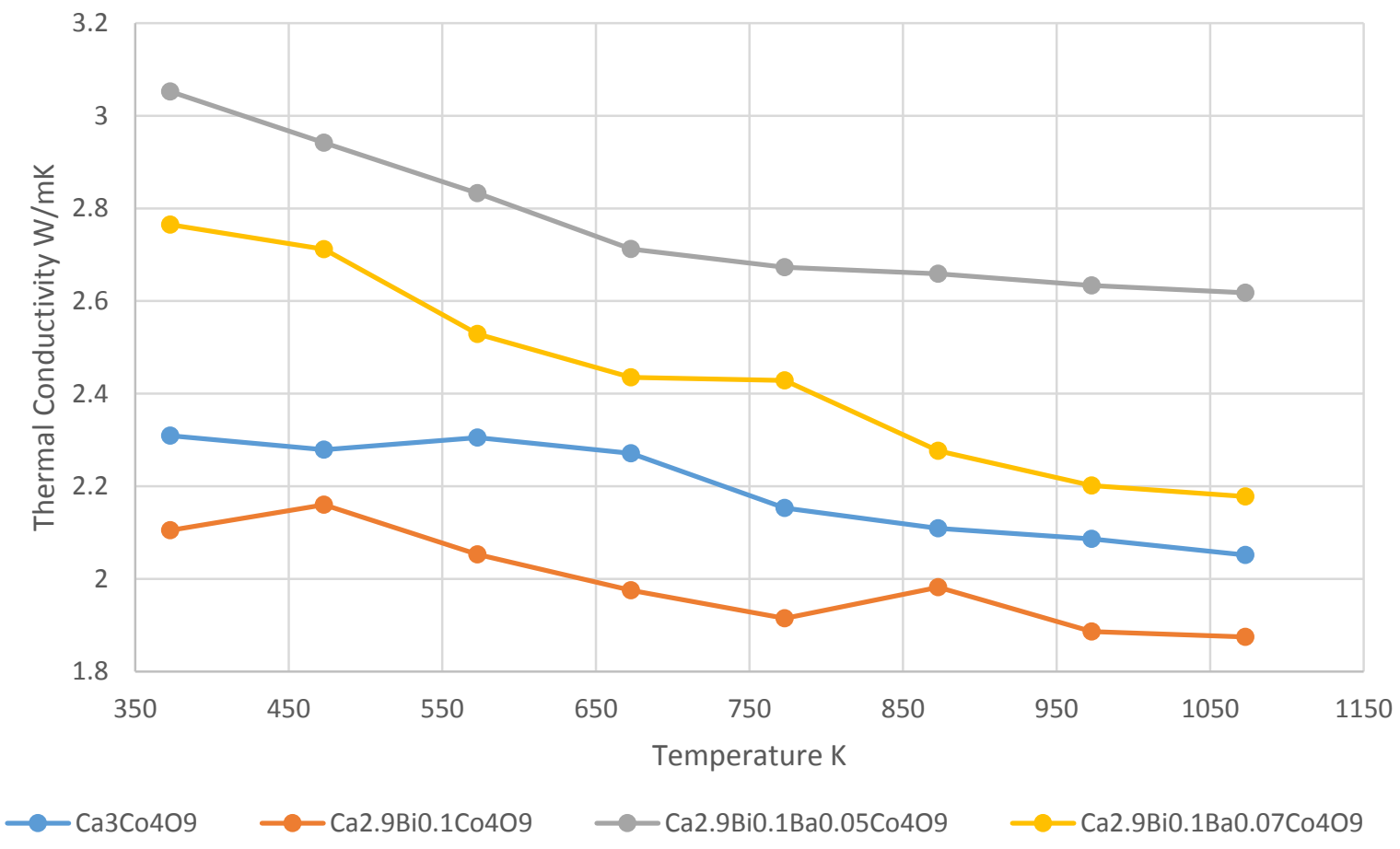

Figure 65 Temperature dependence of thermal conductivity for $\mathrm{Ca}_{2.9} \mathrm{Bi}_{0.1} \mathrm{Ba}_{4} \mathrm{Co}_{4} \mathrm{O}_{9}$ bismuth cation substitution and barium non-stoichiometric addition where $u=0.0,0.05$, and 0.07 . 


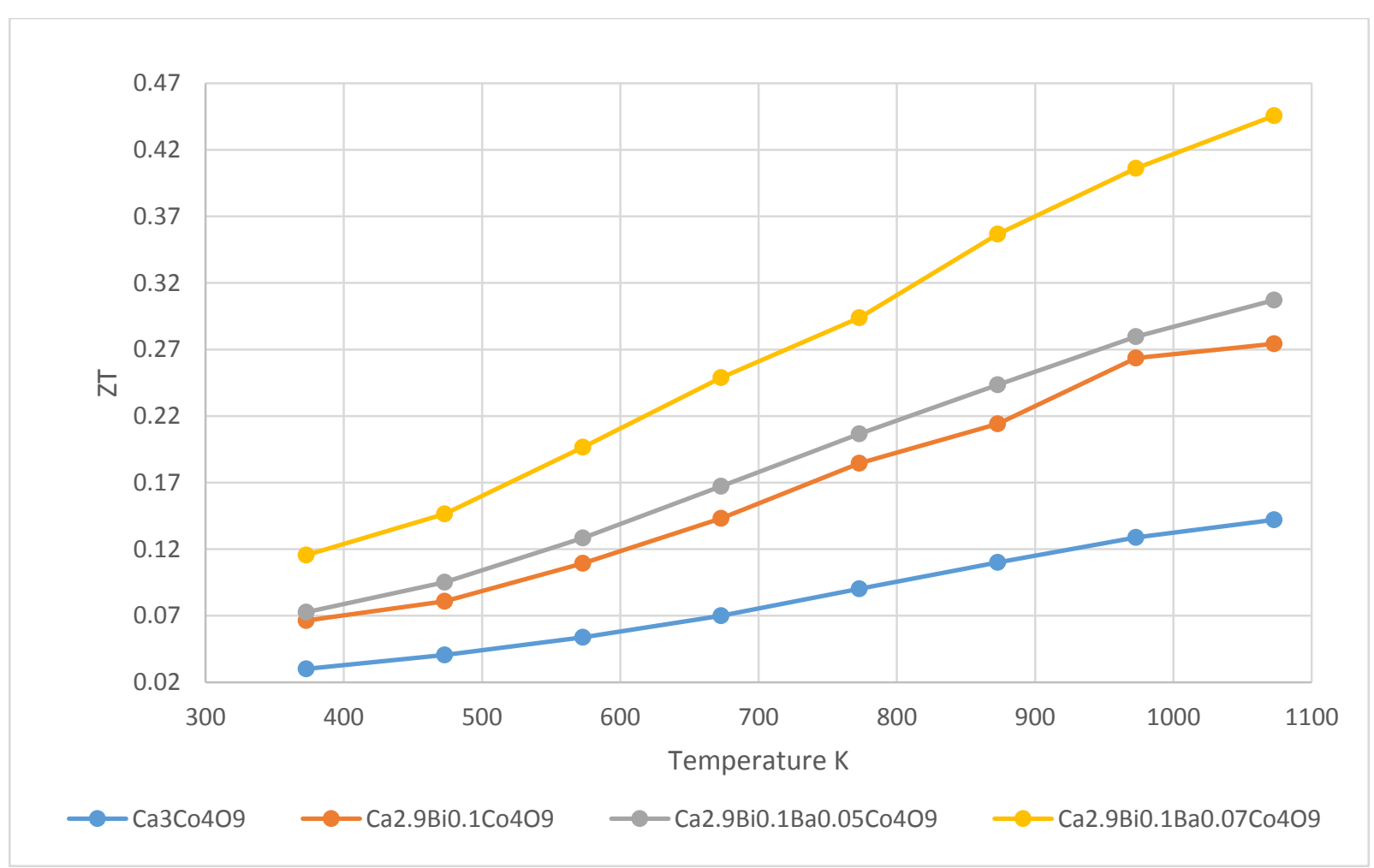

Figure 66 Temperature dependence of figure of merit for $\mathrm{Ca}_{2.9} \mathrm{Bi}_{0.1} \mathrm{Ba}_{4} \mathrm{CO}_{4} \mathrm{O}_{9}$ bismuth cation substitution and barium non-stoichiometric addition where $u=0.0,0.05$, and 0.07 .

\subsection{Summary of results for $\mathrm{Ca}_{3} \mathrm{C}_{4} \mathrm{O}_{9}$ dual doping of bismuth cation substitution \& barium non-stoichiometric addition}

The effects of dual doping $\mathrm{Ca}_{3} \mathrm{Co}_{4} \mathrm{O}_{9}$ with bismuth and barium on the electrical and thermal properties were analyzed and reported. Powders were attained through a combination of cationic substitution and non-stoichiometric ratios mixed through a chemical sol-gel route. The powders were then uniaxially hot pressed and sintered to form the polycrystalline bulk samples. The presence of bismuth at the grain causes the electrical resistivity to decrease drastically at room temperature. Doping with barium 
causes the resistivity to drop further and maintain their low resistivity well into the high temperature regime. The dual doping of bismuth and barium allows the relaxation of the commensurate alternate stacks of the $\mathrm{CoO}$ conductive layer and the $\mathrm{CaCo}_{2} \mathrm{O}_{3}$ insulating layer causing this drastic decrease in electrical resistivity. ${ }^{6]}$

Furthermore, the barium at the grain boundary along with the bismuth acting in tandem as a filter to decrease the carrier mobility results in a large increase in a room temperature Absolute Seebeck Coefficient. With the substitution of bismuth the room temperature Absolute Seebeck Coefficient increases almost 14\% from 120 $\mu \mathrm{V} / \mathrm{K}$ to $\sim 140$ $\mu \mathrm{V} / \mathrm{K}$. The synergistic effect of both at the grain boundary causes the room temperature Absolute Seebeck Coefficient to increase $30 \%$ to $160 \mu \mathrm{V} / \mathrm{K}$. The power factor increases when doped with bismuth and continues to increase with increasing barium up to $u=0.07$. Bismuth and barium do not react at the boundary to co-segregate rather the enrichment of barium aids in the depletion of bismuth at the boundary due to the difference in ionic size. While bismuth's $117 \mathrm{pm}$ ionic radius tends to replace the $114 \mathrm{pm}$ radius of calcium ions in the lattice, barium, with an ionic radius of $149 \mathrm{pm}$, cannot and remains at the boundary to enhance the Absolute Seebeck coefficient. The highest power factor achieved is $\sim 0.94 \mathrm{mWm}^{-1} \mathrm{~K}^{-1}$ at $1005 \mathrm{~K}$ which is one of the highest reported for doped $\mathrm{Ca}_{3} \mathrm{Co}_{4} \mathrm{O}_{9}$. The same sample achieves a ZT of 0.45 at $1073 \mathrm{~K}$. 


\subsection{References}

1 Marat Gunes, Macit Ozenbas. Effect of grain size and porosity on phonon scattering enhancement of $\mathrm{Ca}_{3} \mathrm{Co}_{4} \mathrm{O}_{9}$. Journal of Alloys and Compounds Vol.626, pp.360-367, 2015.

2 Boyle, Cullen; Calvillo, Paulo; Chen, Yun; Barbero, Ever J.; McIntyre, Dustin. Grain boundary segregation and thermoelectric performance enhancement of bismuth doped calcium cobaltite. Journal of the European Ceramic Society 36(3):601-607 Elsevier 2016 0955-2219

3 Masashi Mikami, Kanju Chong, Yuzuru Miyazaki, Tsuyoshi Kajitani, Takahiro Inoue, Satoshi Sodeoka, and Ryoji Funahashi. Bi-Substitution effects on crystal structure and thermoelectric properties of $\mathrm{Ca}_{3} \mathrm{CO}_{4} \mathrm{O}_{9}$ single crystals. Japanese Journal of Applied Physics. Vol.45, No.5A, pp.4131-4136, 2006.

${ }^{4}$ Live Science Staff. Facts about Bismuth. September 5, 2013.

5 Calvillo, Paulo. Chen, Yun. Boyle, Cullen. Barnes, Paul. N. Song, Xueyan. Thermoelectric performance enhancement of calcium cobaltite through barium grain boundary segregation. Inorganic Chemistry. American Chemical Society Publications. September 11, 2015.

${ }^{6}$ Cullen Boyle, Liang Liang, Yun Chen, Jacky Prucz, Ercan Cakmak, Thomas R. Watkins, Edgar Lara-Curzio, Xueyan Song. Competing dopants grain boundary segregation and resultant Seebeck coefficient and power factor enhancement of thermoelectric calcium cobaltite ceramics. Ceramics International. Vol.43, Issue 14, pp.11523-11528, 2017.

7 Ruoming Tian, Tianshu Zhang, Dewei Chu, Richard Donelson, Li Tao, Sean Li. Enhancement of high temperature thermoelectric performance in $\mathrm{Bi}, \mathrm{Fe}$ co-doped layered oxide-based material $\mathrm{Ca}_{3} \mathrm{C}_{4} \mathrm{O}_{9+\delta}$. Journal of Alloys and Compounds. Vol.615, pp.311-315, 2014.

8 Ankam Bhaskar, Z. -R. Lin, Chia-Jyi Liu. Thermoelectric properties of $\mathrm{Ca}_{2.95} \mathrm{Bi}_{0.05} \mathrm{Co}_{4}$ ${ }_{x} \mathrm{Fe}_{x} \mathrm{O}_{9+\delta}(0<\mathrm{x}<0.15)$. Energy Conversion and Management. Vol.76, pp.63-67, 2013.

9 Haoshan Hao, Limin Zhao, and Xing Hu. Microstructure and thermoelectric properties of $\mathrm{Bi}$-and $\mathrm{Cu}$-substituted $\mathrm{Ca}_{3} \mathrm{Co}_{4} \mathrm{O}_{9}$ oxides. Journal of Materials Science technology, Vol.25, pp.105-108, 2009.

${ }^{10}$ Q. M. Lu, J. X. Zhang, Q. Y. Zhang, Y. Q. Liu, and D. M. Liu. Improved thermoelectric properties of $\mathrm{Ca}_{3-\mathrm{x}} \mathrm{Ba}_{x} \mathrm{CO}_{4} \mathrm{O}_{9}(\mathrm{x}=0 \sim 0.4)$ bulks by sol-gel and SPS method. $15^{\text {th }}$ International Conference of Thermoelectrics. 2006.

11 Haoshan Hao, Qinglin He, ChangQing Chen, Hongwei Sun, and Xing Hu. Textured structure and anisotropic thermoelectric properties of $\mathrm{Ca}_{2.7} \mathrm{Bi}_{0.3} \mathrm{Co}_{4} \mathrm{O}_{9}$ oxide prepared by conventional solid-state reaction. International Journal of Modern Physics B. Vol.23, No.1, pp.87-95, 2009. 
12 Zhang Feipeng, Lu Qingmei, Li Tingxian, Zhang Xin, Zhang Jiuxing, Song Xiaoyan. Preparation and thermoelectric transport properties of Ba-, La-, and Ag- doped $\mathrm{Ca}_{3} \mathrm{C}_{4} \mathrm{O}_{9}$ oxide materials. Journal of Rare Earths. Vol.31, No.8, pp.778-783, 2013.

${ }^{13}$ G. Constantinescu, Sh. Rasekh, M. A. Torres, M. A. Madre, A. Sotelo, J. C. Diez. Improvement of thermoelectric properties in $\mathrm{Ca}_{3} \mathrm{Co}_{4} \mathrm{O}_{9}$ ceramics by $\mathrm{Ba}$ doping. Journal of Materials Science., Vol.26, pp.3466-3473, 2015.

14 Siwen Li, Ryoji Funahashi, Ichiro Matsubara, Kazuo Ueno, Satoshi Sodeoka, and Hiroyuki Yamada. Synthesis and thermoelectric properties of the new oxide materials $\mathrm{Ca}_{3-x} \mathrm{Bi}_{x} \mathrm{Co}_{4} \mathrm{O}_{9+\delta}(0.0<\mathrm{x}<0.75)$. Chemical Material, Vol.12, pp.2424-2427, 2000.

${ }^{15}$ Zhang Feipeng, Lu Qingmei, Li Tingxian, Zhang Xin, Zhang Jiuxing, Song Xiaoyan.

${ }^{16}$ T. Sun, H. H. Hng, Q. Y. Yan, and J. Ma. Enhanced high temperature thermoelectric properties of $\mathrm{Bi}$-doped $\mathrm{c}$-axis oriented $\mathrm{Ca}_{3} \mathrm{C}_{4} \mathrm{O}_{9}$ thin films by pulsed laser deposition. Journal of Applied Physics. Vol.108, 2010.

${ }^{17}$ Sajid Butt, Yaoyu Ren, Muhammad Umer Farooq, Bin Zhan, Rizwan ur Rahman Sagar, Yuanhua Lin, Ce-Web Nan. Enhanced thermoelectric performance of heavy-metals ( $M$ $\mathrm{Ba}, \mathrm{Pb})$ doped misfit-layered ceramics: $\left(\mathrm{Ca} 2-\mathrm{x} \mathrm{M}_{\mathrm{x}} \mathrm{CoO}_{3}\right)_{0.62}\left(\mathrm{CoO}_{2}\right)$. Energy Conversion and Management. Vol.83, pp.35-41, 2014.

${ }^{18}$ Guangkun Ren, Jinle Lan, Chengcheng Zeng, Yaochun Liu, Bin Zhan, Sajid Butt, YuanHua Lin, and Ce-Wen Nan. High performance oxides-based thermoelectric materials. Journal of Minerals, Metals \& Materials Society. Vol.67, No.1, pp.211-221, 2015.

19 C. S. Huang, F. P. Zhang, X. Zhang, Q.M. Lu, J. X. Zhang, Z. Y. Liu. Enhanced thermoelectric figure of merit through electrical and thermal transport modulation by dual-doping and texture modulating for $\mathrm{Ca}_{3} \mathrm{C}_{4} \mathrm{O}_{9+\delta}$ oxide materials. Journal of Alloys and Compounds. Vol.687, pp.87-94, 2016.

$20 \mathrm{Zi}$-Hua Wu, Hua-Qing Xie, and Yong-Biao Zhai. Preparation and thermoelectric properties of co-doped ZnO synthesized by sol-gel. Journal of Nanoscience and nanotechnology. Vol.15, pp.3147-3150, 2015. 


\section{Chapter 10: \\ Conclusion and Suggestions for Future Work}

\subsection{Conclusion}

To achieve a high efficiency in a thermoelectric material one must first find a way to decouple the two electrical properties. These properties are the electrical resistivity and the Absolute Seebeck Coefficient. Furthermore, according to the Wiedemann-Franz Law, the thermal conductivity is inversely proportional to these as well. ${ }^{[1,2,3]}$ As the electrical resistivity is enhanced the thermal conductivity suffers. The literature made mention of a Phonon Glass Electron Crystal (PGEC) theory stating that there is potential to create a material that will simulate the phonon capabilities of a glass while carrying out the electrical properties of a perfect crystal. ${ }^{[4,5]}$ Scientist have yet to find the perfect PGEC.

The work in this dissertation shows great promise for the field of oxides as thermoelectric material. Mainly since the decoupling of the electrical properties has been achieved with great results and repeated through more than one different chemistry. It has proven that the electrical conductivity and the Absolute Seebeck Coefficient can be manipulated separately in order to enhance both. It is still uncertain how to intently manipulate the thermal conductivity without directly lowering its density. Although, varying pressing parameters proved to be successful in lowering thermal conductivity without adversely affecting the electrical properties.

The two main new avenues of exploration utilized to achieve these results have been through non-stoichiometric addition and the dual doping of $\mathrm{Ca}_{3} \mathrm{Co}_{4} \mathrm{O}_{9}$ in order to 
engineer the grain boundary while fine tuning the electrical resistivity. In doing so there is more control over where the dopant will deposit itself and how it will act to improve upon the electrical properties, micro-, and nanostructures of the bulk samples based on the properties of the dopant.

Chapter 3 demonstrates the Absolute Seebeck coefficient enhancement through bismuth telluride. Chapter 4 detailed the ability to isolate the bismuth to dope the $\mathrm{Ca}_{3} \mathrm{Co}_{4} \mathrm{O}_{9}$ with successful results of enhancing the Absolute Seebeck coefficient to $\sim 150$ $\mu \mathrm{V} / \mathrm{K}$ at $315 \mathrm{~K}$. Bismuth deposits into the lattice to improve crystal texture and grain alignment causing a reduction in electrical resistivity while its presence at the grain boundary allows for enhancement of the Absolute Seebeck coefficient. Prior to this research the best figure of merit for bismuth cation substitution was reported by Ruomin Tian, et al. with a value of 0.29 at $973 \mathrm{~K}$. Through a sol-gel method and conventional pressing and sintering it has now been enhanced to a value of 0.42 at 973 and $1073 \mathrm{~K} \mathrm{a}$ $45 \%$ increase.

A non-stoichiometric approach was used in chapter 5 to dope the $\mathrm{Ca}_{3} \mathrm{Co}_{4} \mathrm{O}_{9}$ in order to investigate whether the electrical properties can be further improved through this alternative method. Processing parameters were also varied in order to control the pellet's density and obtain the optimum conditions for its thermal conductivity and to balance the carrier mobility and concentration and achieve the best electrical properties. A figure of merit of 0.52 at 973 and $1073 \mathrm{~K}$ was achieved for the $\mathrm{Ca}_{3} \mathrm{Bi}_{0.3} \mathrm{Co}_{4} \mathrm{O}_{9}$ chemistry pressed at $0.75 \mathrm{GPa}$ with a temperature of $423 \mathrm{~K}$. Considering there has been no reports on the non-stoichiometric addition method, comparing it to the cation substitution of bismuth instead demonstrates an $80 \%$ increase at $973 \mathrm{~K}$. 
In order to further analyze the grain boundary segregation experienced in the bismuth sets, barium was used to dope the $\mathrm{Ca}_{3} \mathrm{C}_{4} \mathrm{O}_{9}$ in chapter 6 with this same nonstoichiometric addition method. The motivation was to investigate a dopant that does not have the ability to replace calcium deficiencies and deposits at the grain interior only. This chemistry, like the last two, was also successful in enhancing the room temperature Absolute Seebeck coefficient. Barium allowed for a higher saturation level than that of the bismuth non-stoichiometric chemistry achieving a value of $\sim 160 \mu \mathrm{V} / \mathrm{K}$ at $315 \mathrm{~K}$. In addition, barium, with its semi-conductor-like behavior, also aids in crystal texture development improving grain alignment and connectivity allowing for very promising electrical properties. A high power factor of $0.82 \mathrm{~mW} / \mathrm{mK}^{2}$ at $315 \mathrm{~K}$ and $0.84 \mathrm{~mW} / \mathrm{mK}^{2}$ at $910 \mathrm{~K}$ was achieved with a chemistry of $\mathrm{Ca}_{3} \mathrm{Ba}_{0.05} \mathrm{C}_{04} \mathrm{O}_{9}$.

Dual doping the $\mathrm{Ca}_{3} \mathrm{Co}_{4} \mathrm{O}_{9}$ structure with bismuth and barium allowed for great control over the electrical properties. The chemistry utilizing the cation substitution and non-stoichiometric addition in tandem yielded a high power factor of $0.94 \mathrm{~mW} / \mathrm{mK}^{2}$ at high temperatures with the $\mathrm{Ca}_{2.9} \mathrm{Bi}_{0.1} \mathrm{Ba}_{0.07} \mathrm{C}_{4} \mathrm{O}_{9}$ chemistry. Bismuth's presence in the lattice improved the crystal texture of the $\mathrm{Ca}_{3} \mathrm{C}_{4} \mathrm{O}_{9}$ bulk sample by improving grain alignment and connectivity along with triggering the grain growth. Bismuth's presence at the grain boundary enhanced the Absolute Seebeck coefficient at room temperature and the further doping of barium continued to improve on the grain boundary segregation reaching a value of $\sim 165 \mu \mathrm{V} / \mathrm{K}$ at $315 \mathrm{~K}$. There was no co-segregation at the boundary rather the increasing presence of barium depleted the bismuth's content at the boundary due to the difference in ionic size. This can be seen in the progression of the microstructure development illustrated in figure 67. 

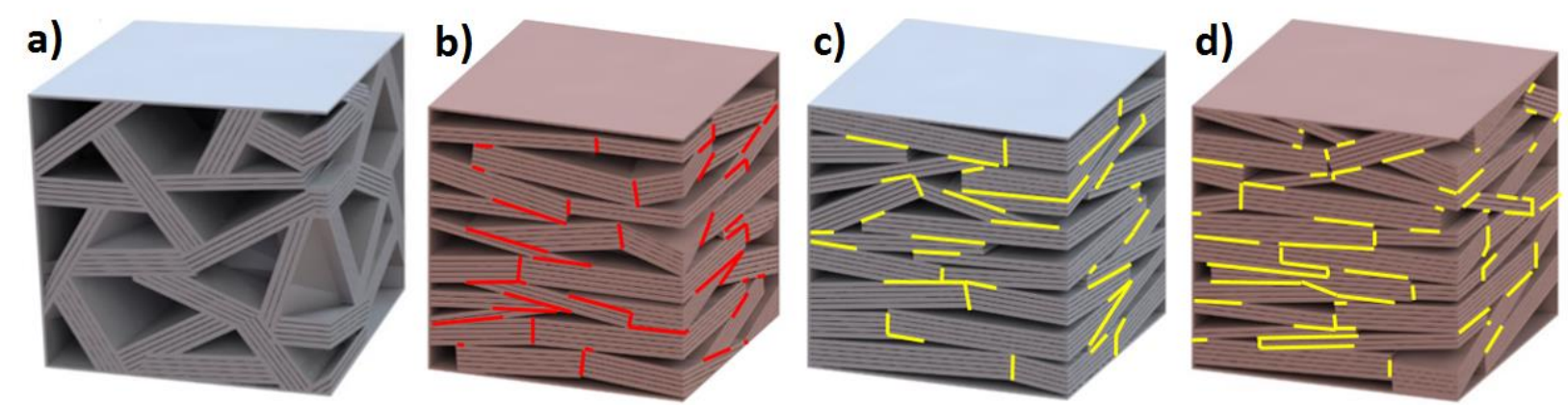

$$
\begin{aligned}
& \mathrm{Ca}_{3} \mathrm{CO}_{4} \mathrm{O}_{9} \\
& \mathrm{Ca}_{3} \mathrm{CO}_{4} \mathrm{O}_{9} \text { grain } \\
& \text { interior }
\end{aligned}
$$$$
\mathrm{Ca}_{2.7} \mathrm{Bi}_{0.3} \mathrm{Co}_{4} \mathrm{O}_{9}
$$$$
\mathrm{Ca}_{3} \mathrm{Ba}_{0.05} \mathrm{Co}_{4} \mathrm{O}_{9}
$$$$
\mathrm{Ca}_{2.9} \mathrm{Bi}_{0.1} \mathrm{Ba}_{0.07} \mathrm{Co}_{4} \mathrm{O}_{9}
$$

\section{Bismuth in grain} interior
Bismuth at grain boundary
Barium at grain boundary

Figure 67 Microstructure development of undoped $\mathrm{Ca}_{3} \mathrm{Co}_{4} \mathrm{O}_{9}, \mathrm{Ca}_{2.7} \mathrm{Bi}_{0.3} \mathrm{Co}_{4} \mathrm{O}_{9}$, $\mathrm{Ca}_{3} \mathrm{Ba}_{0.05} \mathrm{Co}_{4} \mathrm{O}_{9}$, and $\mathrm{Ca}_{2.9} \mathrm{Bi}_{0.1} \mathrm{Ba}_{0.07} \mathrm{CO}_{4} \mathrm{O}_{9}$.

Figure 68 shows the comparison of the temperature dependence of the four best power factors taken from this dissertation. This allows the direct comparison between the doping of the $\mathrm{Ca}_{3} \mathrm{C}_{4} \mathrm{O}_{9}$ with bismuth using the cation substitution and non-stoichiometric addition methods shown in image a) and b). Due to bismuth's unique ability to deposit into the lattice in place of calcium vacancies and at the grain boundary, the nonstoichiometric addition proves to be the more efficient method of the two. The cation substitution method comes close to but never actually cresting a power factor of 1 $\mathrm{mW} / \mathrm{mK}^{2}$ and maintains a consistent value of around $0.8 \mathrm{~mW} / \mathrm{mK}^{2}$ through all temperature ranges. The non-stoichiometric method reaches a power factor of $1.3 \mathrm{~mW} / \mathrm{mK}^{2}$ at low temperatures and maintains a value above $0.9 \mathrm{~mW} / \mathrm{mK}^{2}$ through all temperature ranges.

The $\mathrm{Ca}_{3} \mathrm{C}_{4} \mathrm{O}_{9}$ doped with barium utilizing the non-stoichiometric addition method shown in image c) has a very similar trend to the bismuth cation substitution. It yields a consistent trend with a value of around $0.8 \mathrm{~mW} / \mathrm{mK}^{2}$ through all temperature ranges. The 
dual doping of these two elements was not as straightforward as combining the best two chemistries, along with the best two methods. Bismuth utilized the cation substitution method so when barium enriched the grain boundaries bismuth was able to replace the calcium vacancies thus reducing the chances of an over doped scenario. $\mathrm{Ca}_{3} \mathrm{C}_{4} \mathrm{O}_{9}$ doped with bismuth cation substitution and barium non-stoichiometric addiction is shown in image d) of figure 68. Although its highest power factor does not surpass that of the bismuth non-stoichiometric addition alone, at low temperatures, it achieves similar values throughout the rest of the temperature spectrum. 

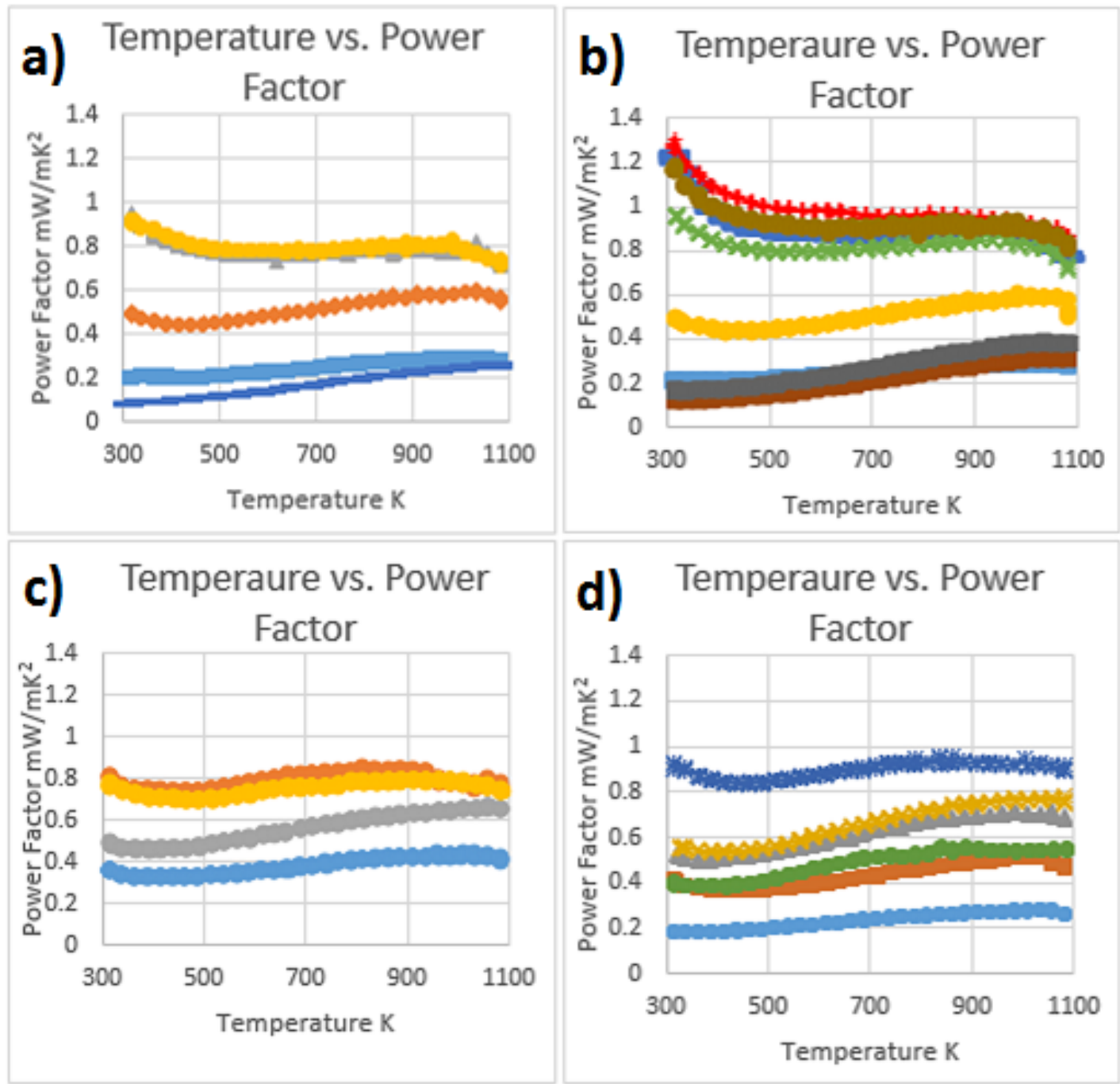

Figure 68 Comparisons of temperature dependence of power factor between a) $\mathrm{Ca}_{2.8} \mathrm{Bi}_{0.2} \mathrm{Co}_{4} \mathrm{O}_{9}$, b) $\mathrm{Ca}_{3} \mathrm{Bi}_{0.3} \mathrm{Co}_{4} \mathrm{O}_{9}$, c) $\mathrm{Ca}_{3} \mathrm{Ba}_{0.05} \mathrm{Co}_{4} \mathrm{O}_{9}$, and d) $\mathrm{Ca}_{2.9} \mathrm{Bi}_{0.1} \mathrm{Ba}_{0.07} \mathrm{Co}_{4} \mathrm{O}_{9}$.

Figure 69 shows the comparison of the temperature dependence of the four best figure of merits taken from this dissertation. Image a) and b) show the direct comparison of figure of merits between the $\mathrm{Ca}_{3} \mathrm{C}_{4} \mathrm{O}_{9}$ doping of bismuth utilizing the cation substitution and non-stoichiometric addition methods. Image b) shows an improvement of figure of merit, from 0.42 in image a) to 0.45 in b), by using the non-stoichiometric addition method. Both values were measured at $1073 \mathrm{~K}$. This is further improved to 0.52 at $1073 \mathrm{~K}$ by varying the pressing parameters from $1 \mathrm{GPa}$ to $0.75 \mathrm{GPa}$. 
The $\mathrm{Ca}_{3} \mathrm{C}_{4} \mathrm{O}_{9}$ doped with barium utilizing the non-stoichiometric addition method shown in image c) was able to match this figure of merit with a value of 0.53 at $1073 \mathrm{~K}$. Although the dual doped set did not surpass the figure of merit of either the bismuth or barium individual non-stoichiometric addition sets, due to its electrical results it holds great promise. The bulk sample's density caused the material to have a fairly large thermal conductivity limiting its figure of merit to 0.45 at $1073 \mathrm{~K}$.
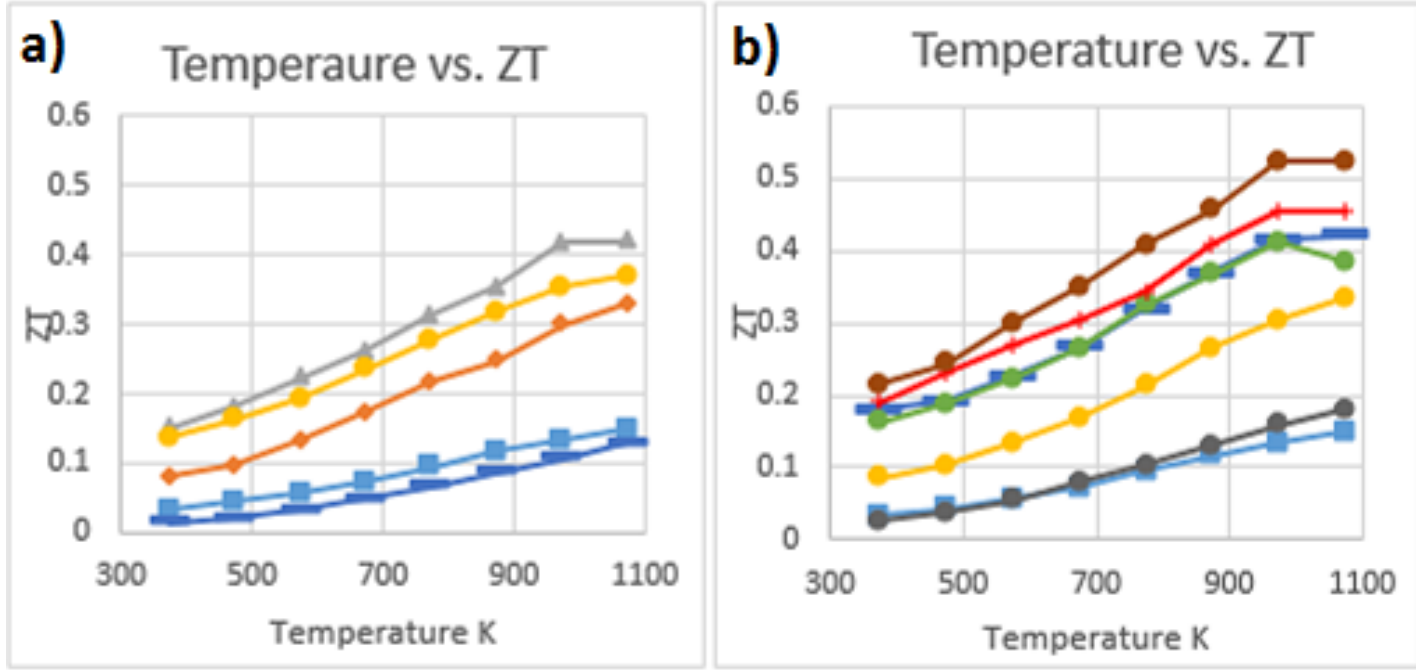

\section{c) Temperature vs. ZT}
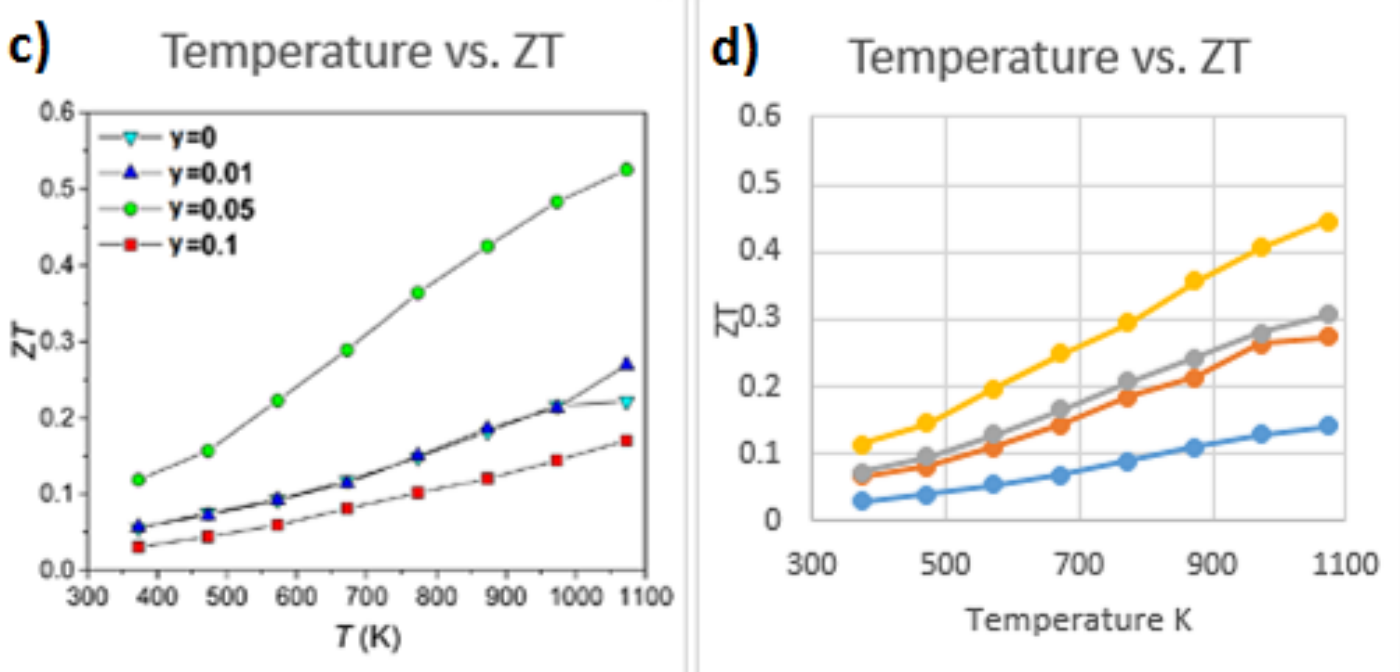

Figure 69 Comparisons of temperature dependence of figure of merit between a) $\mathrm{Ca}_{2.8} \mathrm{Bi}_{0.2} \mathrm{CO}_{4} \mathrm{O}_{9}$, b) $\mathrm{Ca}_{3} \mathrm{Bi}_{0.3} \mathrm{Co}_{4} \mathrm{O}_{9}$, c) $\mathrm{Ca}_{3} \mathrm{Ba}_{0.05} \mathrm{Co}_{4} \mathrm{O}_{9}$, and d) $\mathrm{Ca}_{2.9} \mathrm{Bi}_{0.1} \mathrm{Ba}_{0.07} \mathrm{Co}_{4} \mathrm{O}_{9}$. 


\subsection{Suggestions for future work}

This work leaves great hope within this specific field and topic. It motivates in the search for other dopants that can potentially segregate at the boundary as bismuth and barium both have already shown. It opens the door to finding a dopant that can hopefully enhance the Absolute Seebeck coefficient beyond what barium has already done at low temperatures and possibly allow it to exceed barium's results at high temperatures. With the ability of dual doping there is hope to continue to treat the electrical resistivity and Absolute Seebeck coefficient as two separate entities when looking to alter their properties. The next step for the optimal chemistry involved with the dual doping of $\mathrm{Ca}_{3} \mathrm{C}_{4} \mathrm{O}_{9}$ would be to vary the pressing parameters as was performed in chapter 5 in regards to the bismuth non-stoichiometric addition chemistry set. By attempting to maintain or improve the electrical properties there is the possibility of experiencing a significant drop in thermal conductivity in the same way $\mathrm{Ca}_{3} \mathrm{Bi}_{3}{ }_{3} \mathrm{Co}_{4} \mathrm{O}_{9}$ did, in order to enhance its ZT above its already impressive value of 0.45 at $1073 \mathrm{~K}$.

Although the electrical resistivities in this work have demonstrated very low values there is always hope for further enhancement. This coupled with a high Absolute Seebeck coefficient will yield a promising power factor. Lastly, learning how better to lower the thermal conductivity without negatively effecting these electrical properties will continue to aid in ZT enhancement. As the Wiedemann-Franz Law states as long as the lattice thermal conductivity is lowered the electrical properties should have minimal effect on its results. ${ }^{[1,2,3]}$ This gives hope for this last parameter, the thermal conductivity, to help achieve a desirable figure of merit. One that can allow this material to be used on a large scale. 


\subsection{References}

1 Guodong Tang, Wenchao Yang, Jianfeng Wen, Zhuangchun Wu, Cang Fan, zhihe Wang. Ultralow thermal conductivity and thermoelectric properties of carbon nanotubes doped $\mathrm{Ca}_{3} \mathrm{C}_{4} \mathrm{O}_{9+\delta}$. Ceramics International 41, pp. 961-965. September 15, 2014.

2 S. Demirel, E. Altin, E. Oz, S. Altin, A. Bayri. An enhancement ZT and spin state transition of $\mathrm{Ca}_{3} \mathrm{Co}_{4} \mathrm{O}_{9}$ with $\mathrm{Pb}$ doping. Journal of Alloys and Compounds 627, pp. 430437, December 27, 2014.

${ }^{3}$ A. Sotelo, F. M. Costa, N. M. Ferreira, A. Kovalevsky, M. C. Ferro, V. S. Amaral, J. S. Amaral, Sh. Rasekh, M. A. Torres, M. A. Madre, J. C. Diez. Tailoring $\mathrm{Ca}_{3} \mathrm{Co}_{4} \mathrm{O}_{9}$ microstructure and performances using a transient liquid phase sintering additive. Journal of the European Ceramic Society 36, pp. 1025-1032, November 30, 2015.

${ }^{4}$ Materials Science. Division of Engineering \& Applied Science. California Institute of Technology. 2017.

${ }^{5}$ Chong Xiao, Jie Xu, Boxiao Cao, Kun Li, Mingguang Kong, and Yi Xie. Solid-solutioned homojunction nanoplates with disordered lattice: A promising approach toward "Phonon Glass Electron Crystal" thermoelectric materials. Journal of the American Chemical Society 134, pp. 7971-7977, April 23, 2012. 


\section{Publications}

1. Cullen Boyle, Paulo Cavillo, Yun Chen, Ever J. Barbero, Dustin McIntyre, Xueyan Song, "Grain Boundary Segregation and Thermoelectric Performance Enhancement of Bismuth Doped Calcium Cobaltite", Journal of the European Ceramic Society, Vol. 36, Issue 3 (2016)-pp.601-607

2. Paulo Cavillo, Yun Chen, Cullen Boyle, Paul N. Barnes, and Xueyan Song, "Thermoelectric Performance Enhancement of Calcium Cobaltite through Barium Grain Boundary Segregation", Inorganic Chemistry, 54 (2015) 9027-9032

3. Cullen Boyle, Liang Liang, Yun Chen, Jacky Prucz, Ercan Cakmak, Thomas R. Watkins, Edgar Lara-Curzio, and Xueyan Song, "Competing Dopants Grain Boundary Segregation and Resultant Seebeck Coefficient and Power Factor Enhancement of Thermoelectric Calcium Cobaltite Ceramics", Ceramics International, (2017)

4. Cullen Boyle, Liang Liang, Cesar-Octavio Romo-De-La-Cruz, Robert Johnson, Yun Chen, Jacky Prucz, Ercan Cakmak, Thomas R. Watkins, Edgar Lara-Curzio, Xueyan Song, "Improving Thermoelectric Performance and Thermal Stability of $\mathrm{Ca}_{3} \mathrm{C}_{4} \mathrm{O}_{9}$ Ceramics through Sintering in Oxygen Atmosphere" (submitted) 